\title{
Experimental and Modeling Study of Sliding Wear Performance for Selected Molybdenum Stellite Alloys
}

By

\section{Rachel Collier}

A thesis submitted to the Faculty of Graduate and Postdoctoral Affairs in partial fulfillment of the requirements for the degree of

Master of Applied Science

in

Materials Engineering

Carleton University

Ottawa, Ontario

(C)2019, Rachel Collier 


\section{Abstract}

This thesis presents an experimental and modeling study of wear performance of molybdenumcontaining Stellite alloys. The wear testing conditions are of the pin-on-disc type using a tungsten carbide (WC) ball against the Stellite alloys in dry-sliding mode at ambient temperature. Three variables are explored; they are (i) two rotational speeds (50 rpm and $60 \mathrm{rpm}$ ) of the WC ball, (ii) two normal loads ( $15 \mathrm{~N}$ and $25 \mathrm{~N}$ ) on the system, and (iii) various time durations ( $1 \mathrm{hr}$ up to 60 hrs). In addition to the standard wear condition of running a continuous test, another factor of interrupting the test at various intervals is introduced. It is found that by removing the alloy specimen to take interval measurements, and then replacing the same specimen back into the system, the wear loss reduces across each of the alloys under all conditions. Phase evaluation for each of the alloys is undertaken using SEM, EDX and XRD analysis techniques. This reveals the complexity of the microstructures in terms of the various carbides and intermetallics that could be present in each alloy. The volume fraction of carbides is the principal attribute of the Stellite alloys. An evaluation of the volume fraction of carbides reveals Stellite 21 to have $\sim 4 \%$, Stellite 720 to have $\sim 70 \%$, and Stellite 728 to have $\sim 21 \%$. An attempt is made to analyze the particle size using ImageJ and elements contained within the debris using SEM/EDX. This proves challenging to accomplish and more work is required in this area. A wear rate model is developed for these alloys under dry-sliding test conditions. It describes well the experimental data trend and shows how hardness is a significant factor in the wear of molybdenum Stellite alloys. The model sheds light into prediction of long-term wear loss. 


\section{Acknowledgements}

It is with great pleasure and sincerity that I take this opportunity to thank many people who made this thesis possible.

First and foremost, I would like to thank Professor Rong Liu for her commitment to my education and research during my time at Carleton University. Her helpfulness, guidance, and understanding has been paramount to achieving what I have today.

Dr. Xijia $\mathrm{Wu}$, for his assistance and guidance through the modeling section and feedback on the many other sections. I hope the reading didn't "wear" him out too much.

Dr. Matthew X. Yao of Kennametal Stellite, I would not be where I am today without his support as a colleague and friend. I will always be grateful to have worked with him over the past ten plus years. As I always say, "thank you, Matthew".

Dr. Xiaozhou (Joe) Zhang, I could not ask for a more supportive friend. His help, understanding, guidance, and most importantly his friendship has meant a lot to me, and I'm very grateful to have worked alongside him the past six plus years.

To Judith Smith, my friend, neighbor, confidant, babysitter, and most importantly the sternest advocate I had to help me achieve anything I set out to do.

I would also like to thank my employers, Kennametal Stellite Inc., and the Government of Canada for supporting my ambition to always try to better myself, and allowing me to use my new skills in the workplace.

Last but not least, I would like to thank my husband Jason, and my children Isabelle and Michael. It has been a long road with many detours, some good, some not so good, but thank you for walking the road with me. I will be forever grateful of your love and support. 


\section{Table of Contents}

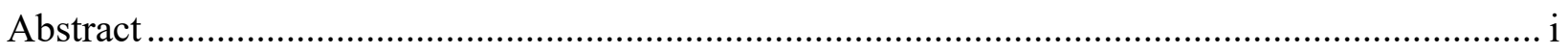

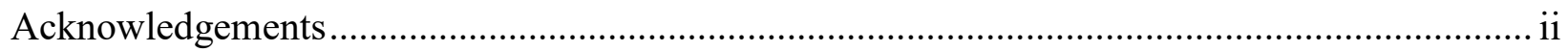

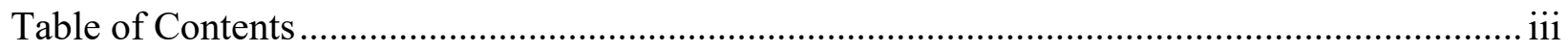

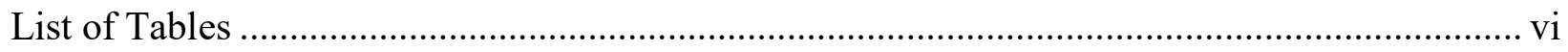

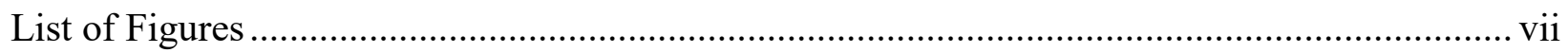

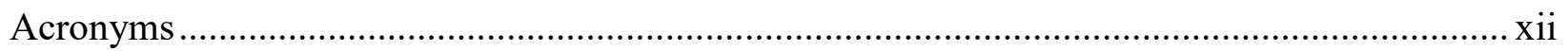

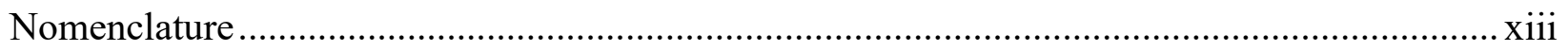

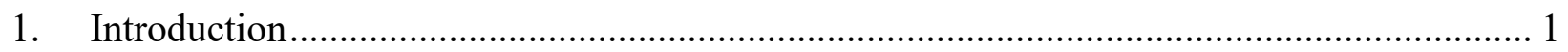

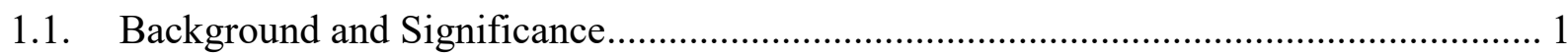

1.1.1. Overview of Stellite Alloys .......................................................................... 1

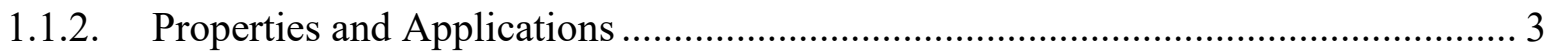

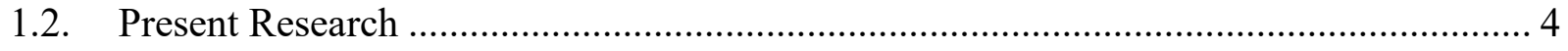

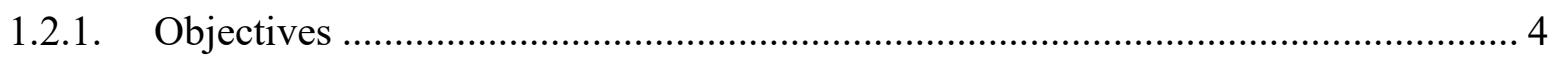

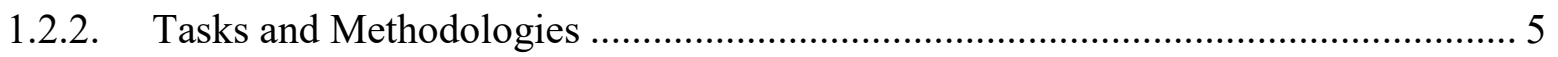

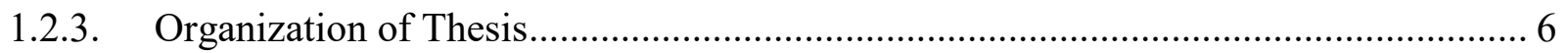

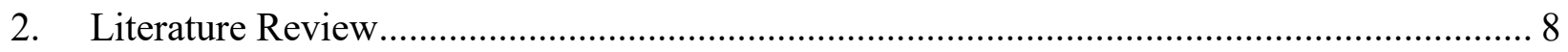

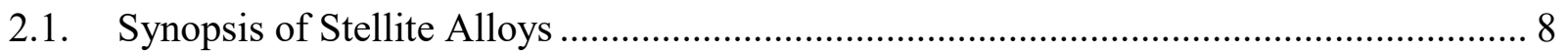

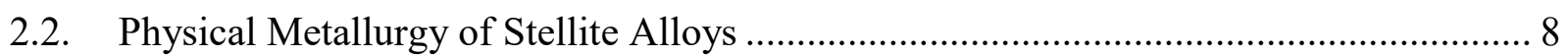

2.2.1 Carbon Content and Carbides ............................................................................. 10

2.2.2 Effects of Processing on Microstructure ....................................................................... 14

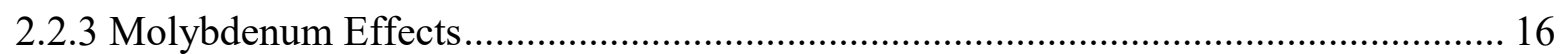

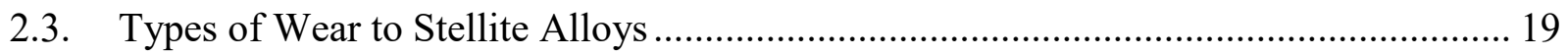

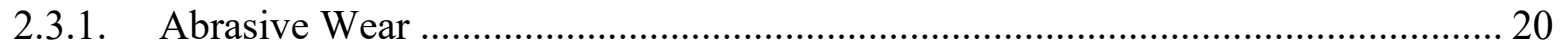

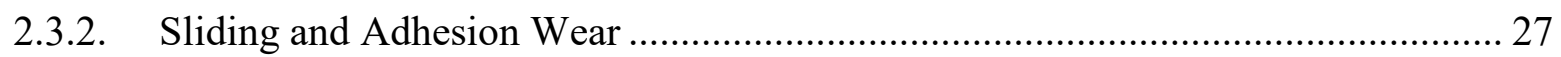

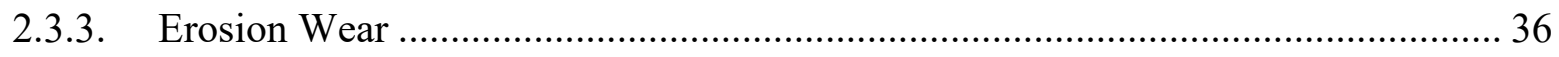

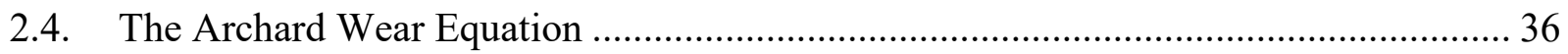

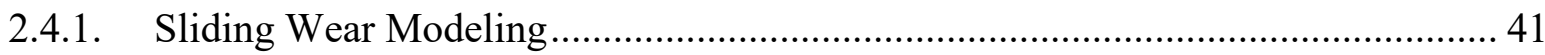

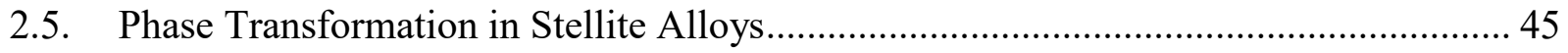

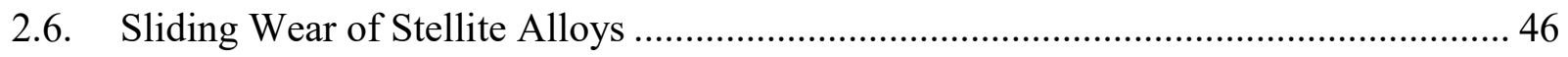

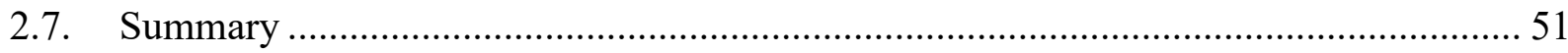

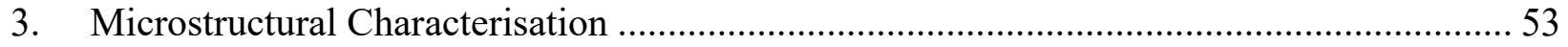

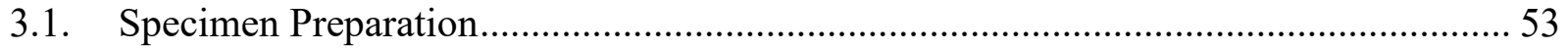

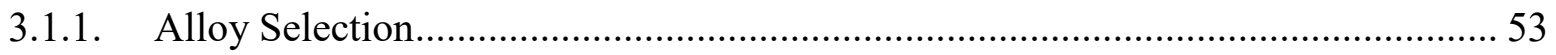




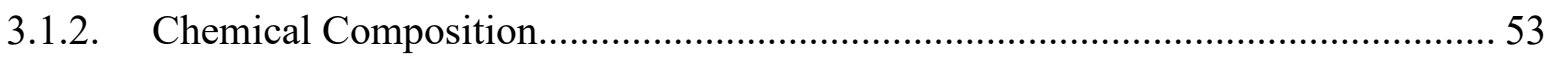

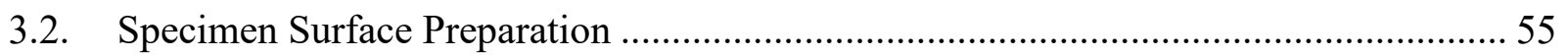

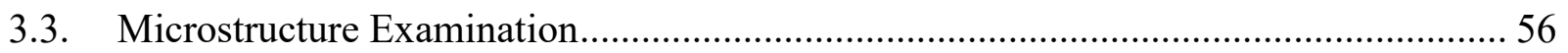

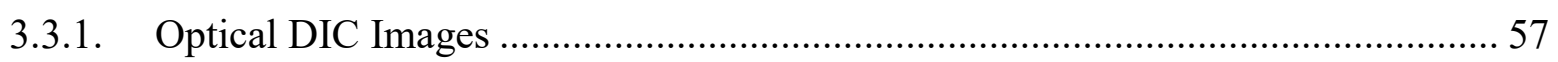

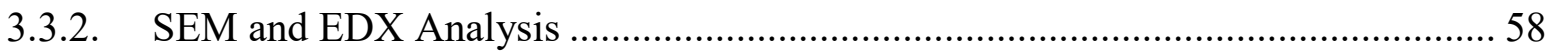

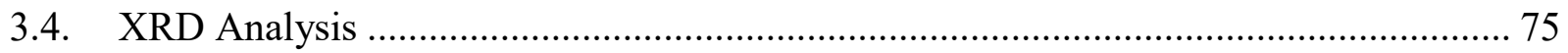

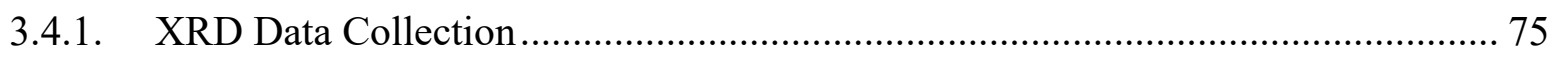

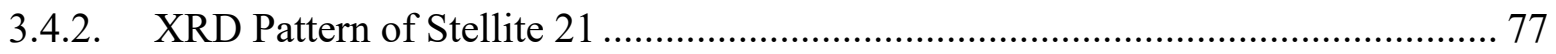

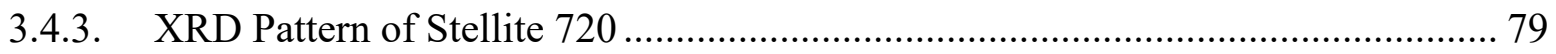

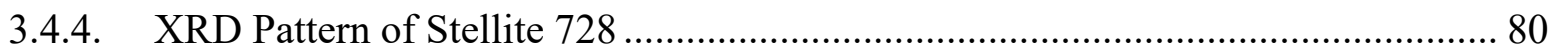

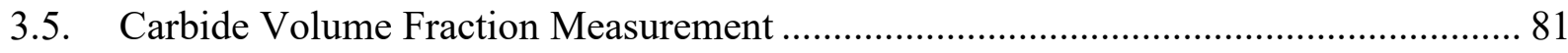

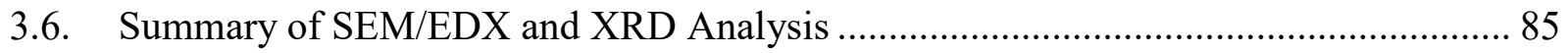

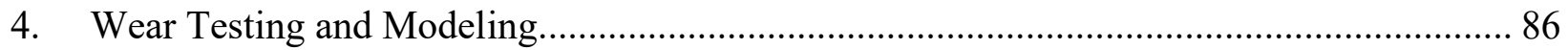

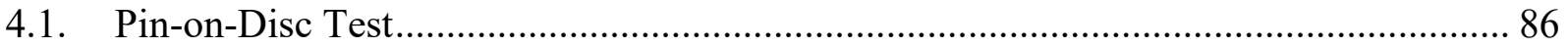

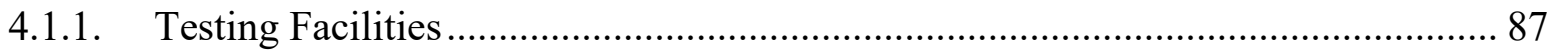

4.1.2. Test Parameters and Data Collection...................................................................... 91

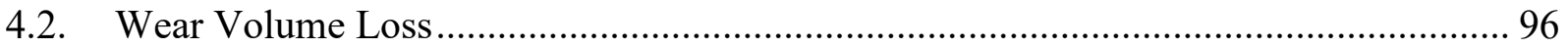

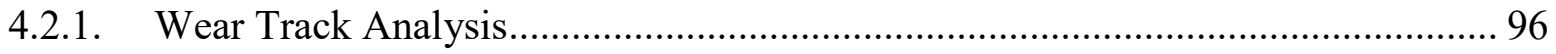

4.2.2. Wear Loss from Continuous Testing at $50 \mathrm{rpm}$ under $15 \mathrm{~N}$ and $25 \mathrm{~N}$................... 98

4.2.3. Wear Volume Loss from Interrupted Testing at $50 \mathrm{rpm}$ under $25 \mathrm{~N}$.................... 100

4.2.4. Comparison of Interrupted and Continuous Testing at $50 \mathrm{rpm}$ under $25 \mathrm{~N}$......... 102

4.1. Friction Coefficient ....................................................................................... 104

4.1.1. Friction Coefficient Analysis for Continuous Testing at $50 \mathrm{rpm}$ under $15 \mathrm{~N}$ and 25 $\mathrm{N} \quad 105$

4.1.2. Friction Coefficient Analysis Comparison of Interrupted and Continuous Testing at $50 \mathrm{rpm}$ under $25 \mathrm{~N}$

4.1.3. Friction Coefficient Comparison of Interrupted Testing at $50 \mathrm{rpm}$ and $60 \mathrm{rpm}$ under $25 \mathrm{~N} \quad 107$

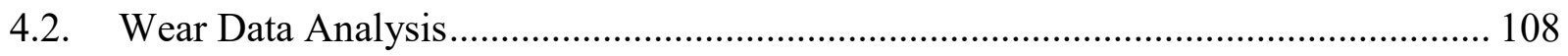

4.2.1. Steady-State Wear and the Archard Equation .................................................. 108

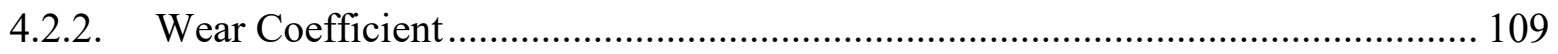

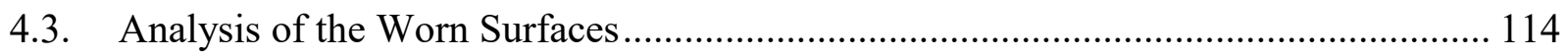

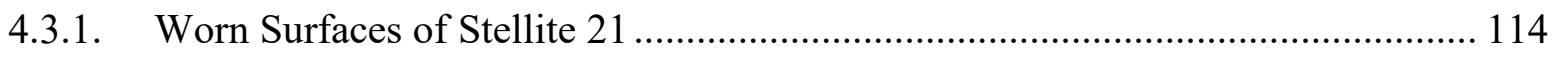

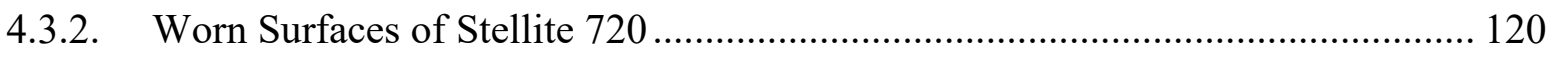

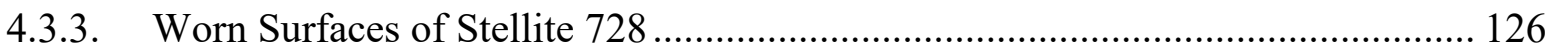




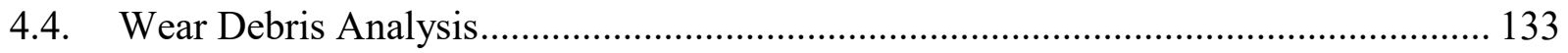

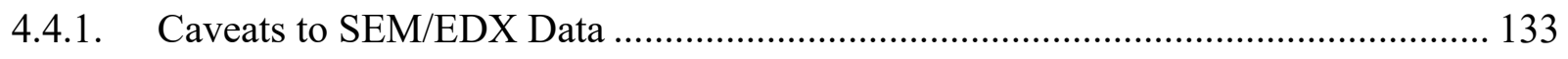

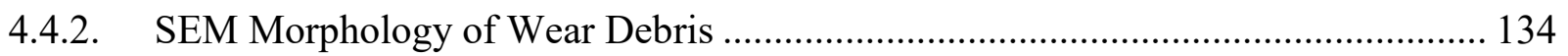

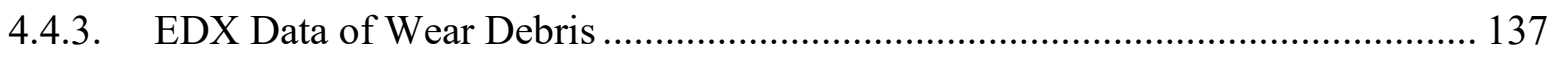

4.4.4. Wear Debris Particle Size Measurement ................................................................ 139

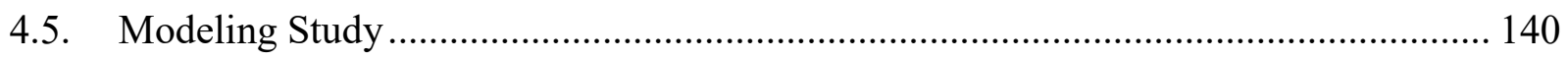

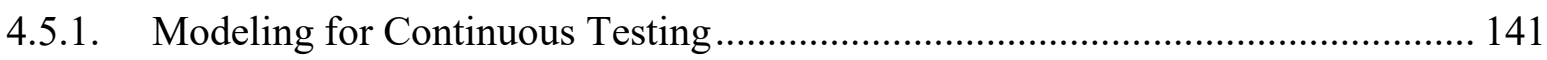

4.5.2. Modeling for Interrupted Testing.................................................................. 145

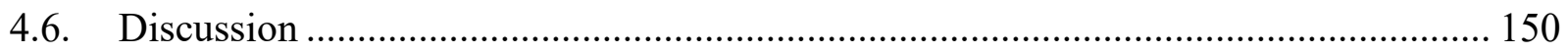

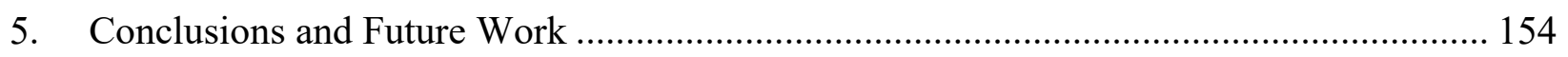

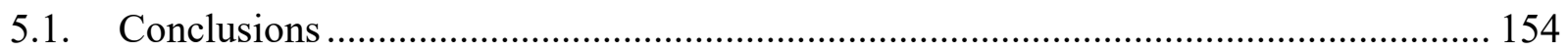

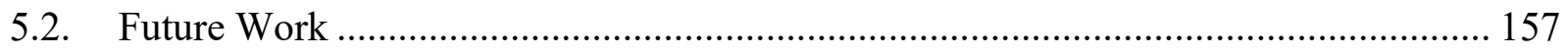

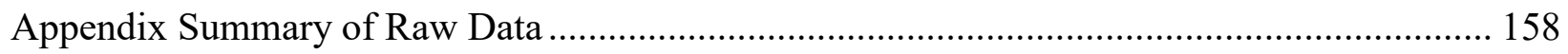

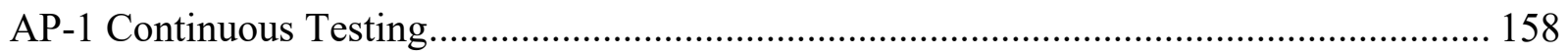

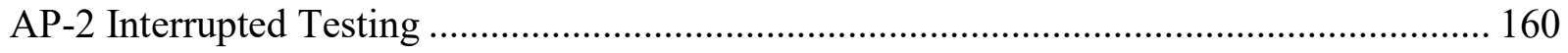

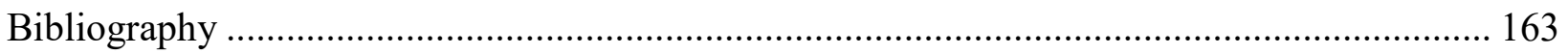




\section{List of Tables}

Table 2-1: Chemical compositions (wt.\%) of common Stellite alloys [2]............................... 9

Table 2-2: Coefficients of friction obtained during adhesive test [32] ................................... 48

Table 2-3: Summary of volume fractions (pct) of each phase in Stellite Alloys [3] ................. 50

Table 3-1: Chemical compositions of selected Stellite Alloys ............................................... 54

Table 3-2: Volume fractions of carbides and intermetallics from the selected Stellite alloys..... 84

Table 4-1: A summary of wear test parameters ............................................................ 91

Table 4-2: Test matrix showing three variables and method of data collection ........................ 92

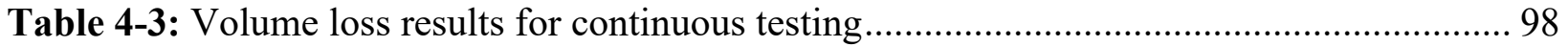

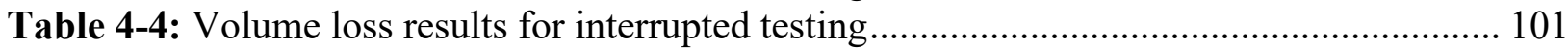

Table 4-5: Hardness test results for the Stellite alloys ......................................................... 110

Table 4-7: Average particle size $(\mu \mathrm{m})$ of wear debris from Stellite 21 specimen in interrupted

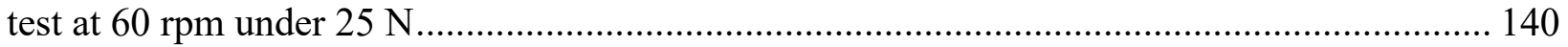

Table 4-8: Mode constants best-fit data for continuous testing ......................................... 143

Table 4-9: Model constants best-fit data for interrupted testing .......................................... 147

Table AP- 1: Raw data of wear track measurements for Stellite 21 continuous tests $50 \mathrm{rpm} . . .158$ Table AP- 2: Raw data of wear track measurements for Stellite 720 continuous tests $50 \mathrm{rpm} .158$ Table AP- 3: Raw data of wear track measurements for Stellite 728 continuous tests $50 \mathrm{rpm} .158$ Table AP- 4: Summary of volume loss for all continuous tests $50 \mathrm{rpm}$............................... 159

Table AP- 5: Raw data of wear track measurements for Stellite 21 interrupted tests $50 \mathrm{rpm}$... 160 Table AP- 6: Raw data of wear track measurements for Stellite 720 interrupted tests $50 \mathrm{rpm} .160$ Table AP- 7: Raw data of wear track measurements for Stellite 728 interrupted tests 50rpm.. 161 Table AP- 8: Raw data of wear track measurements for Stellite 21 interrupted tests $60 \mathrm{rpm} . . .161$ Table AP- 9: Raw data of wear track measurements for Stellite 720 interrupted tests $60 \mathrm{rpm} .162$ Table AP- 10: Raw data of wear track measurements for Stellite 728 interrupted tests 60rpm 162 


\section{List of Figures}

Figure 2-1: Stellite 6 cast microstructure (Figure courtesy of Kennametal Inc.)..................... 11

Figure 2-2: Stellite 20 cast microstructure (Figure courtesy of Kennametal Inc.). .................. 11

Figure 2-3: Vertical section of pseudoternary $(\mathrm{C}, \mathrm{Co}, \mathrm{Cr}+\mathrm{W})$ system. $\mathrm{L}$ is liquid, $\mathrm{M}$ represents

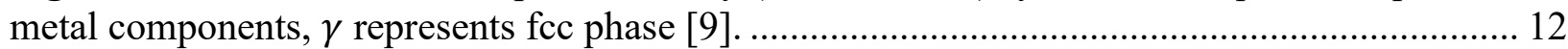

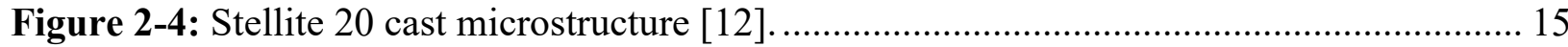

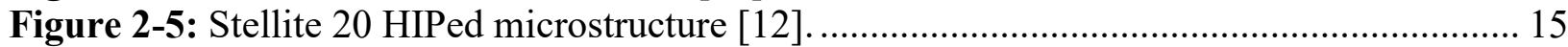

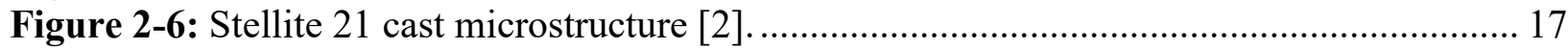

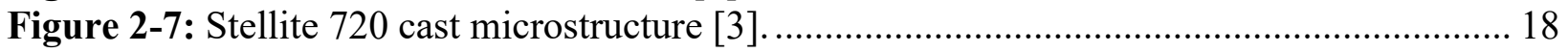

Figure 2-8: Stellite 720 cast microstructure at higher magnification [3] .............................. 18

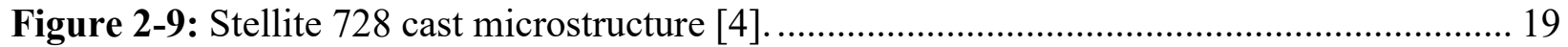

Figure 2-10: Illustration of abrasive wear mechanism [13] ............................................... 20

Figure 2-11: Two types of abrasive wear: (a) two-body abrasion, (b) three-body abrasion [13].20

Figure 2-12: Abrasive wear physical processes [14] ................................................... 21

Figure 2-13: Abrasive wear versus hardness schematic [14] ........................................... 22

Figure 2-14: Interactions of sliding hard or soft abrasive particles and carbides [13]............. 25

Figure 2-15: Abrasive wear versus wt. $\%$ of $\mathrm{M}_{7} \mathrm{C}_{3}$ carbides for sand and graphite cast Co- and

Fe-based alloys $[13]$...................................................................................................... 26

Figure 2-16: Severely worn surface of a $\mathrm{Cu}-10 \mathrm{wt} \% \mathrm{Zn}$ alloy disc when sliding against an AISI

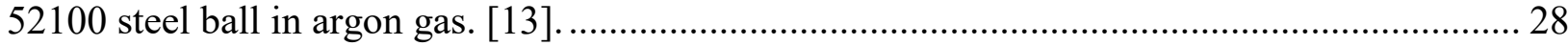

Figure 2-17: Various modes of relative sliding motion and contact geometry [13]................ 29

Figure 2-18: Schematic plot of wear loss as a function of time or sliding distance (general

progress) [14] .................................................................................................. 30

Figure 2-19: Wear loss as a function of time or sliding distance showing the different features of

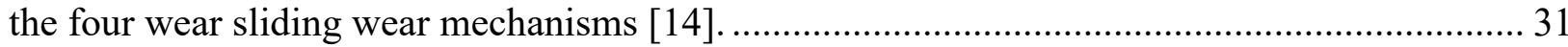

Figure 2-20: Factors influencing wear mechanisms during sliding contact [14].................. 31

Figure 2-21: Metallurgical properties influencing sliding wear [14]. .................................. 32

Figure 2-22: Coefficient of adhesion versus hardness of metals [14] ................................... 33

Figure 2-23: SEM Micrograph of Incoloy MA956 worn surface against a Stellite 6 counterfac at

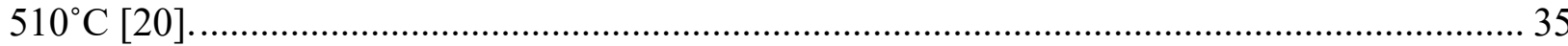

Figure 2-24: Idealized representation of single contact in sliding surfaces, side view (above) and

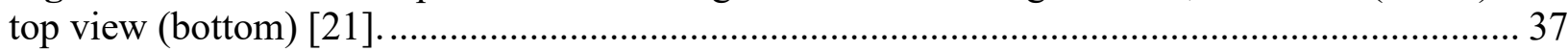

Figure 2-25: Wear vs. sliding distance for pins rubbing on rings of different materials (speed

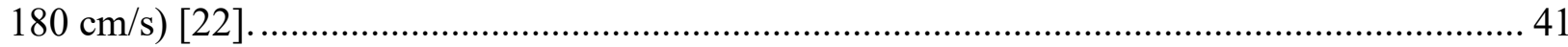

Figure 2-26: Predictions from the GIWM in comparison with the experimental results [25].... 42 Figure 2-27: Comparison of theoretical predictions (solid line) with experimental results (shaped points) of steel disc wear at different loads [27]. 44

Figure 2-28: Comparison of theoretical predictions (solid line) with experimental results (shaped points) of composite pin wear at a normal load of $150 \mathrm{~N}$ [27] ........................................... 45

Figure 2-29: Dry sliding wear map for Stellite 6 against a WC-Co 90/10 disc [31] ................ 47 Figure 2-30: Adhesive-wear resistance of Stellite alloys, ASTM G77, S10 type ring and test

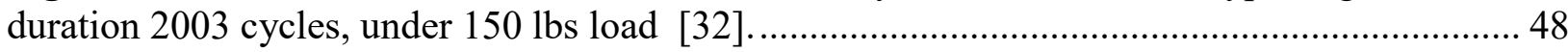

Figure 2-31: Sliding wear resistance of 700 series Stellite alloys, Pin-on-Disc WC Ball, 10 N,

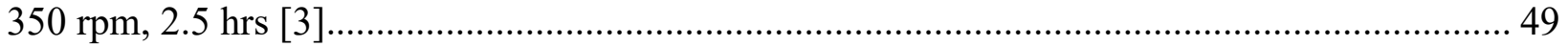


Figure 2-32: Comparison of hardness between CoCrMo and CoCrW Stellite alloys [3]......... 49 Figure 2-33: Comparison of wear resistance between CoCrMo and CoCrW Stellite alloys[3].. 50 Figure 3-1: Struers Cito-Press for hot resin mounting (Struers Inc) .................................... 55 Figure 3-2: Struers Tegramin for Griding and Polishing Mounted Samples (Struers Inc)......... 56

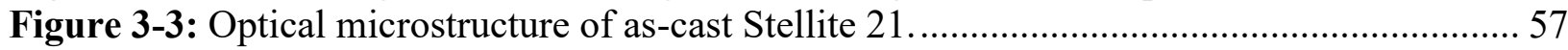

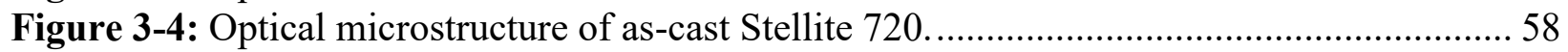

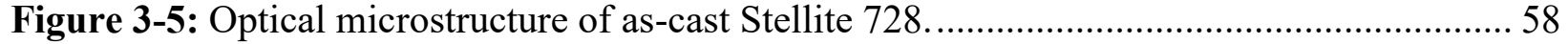

Figure 3-6: Detail of Stellite 21 microstructure using BSEs. ............................................... 60

Figure 3-7: EDX analysis of as-cast Stellite 21 microstructure (a) locations for point analysis, (b) spectrum \#33 dark grey (solid solution), (c) spectrum \#34 coarser eutectic carbide area, (d)

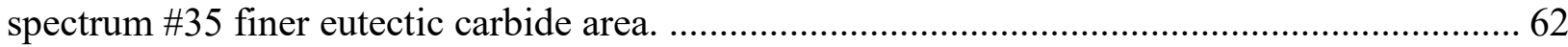

Figure 3-8: EDX map of as-cast Stellite 21 carbide phase (a) image used for EDX map, (b) individual element maps.

Figure 3-9: SEM microstructure of Stellite 720 using BSE image (a) lower magnification, (b) higher magnification.

Figure 3-10: EDX analysis of as-cast Stellite 720 (a) locations for point analysis, (b) spectrum \#28 white phase, (c) spectrum \#29 white phase (second location), (d) spectrum \#30 light grey, (e) spectrum \#31 medium grey, (f) spectrum \#32 dark grey.................................................. 68 Figure 3-11: EDX map of as-cast Stellite 720 (a) EDX map image, (b) individual element maps.

Figure 3-12: SEM microstructure of Stellite 728 using BSE image...................................... 70 Figure 3-13: EDX analysis of as-cast Stellite 728 microstructure (a) locations for point analysis, (b) spectrum \#37 dark grey (solid solution), (c) spectrum \#38 white phase, (d) spectrum \#39 light grey, (e) spectrum \#40 dark grey (solid solution)........................................................ 73 Figure 3-14: EDX map of as-cast Stellite 728 (a) EDX map image, (b) individual element maps.

Figure 3-15: Combined XRD patterns for all three alloys................................................. 76

Figure 3-16: XRD pattern and peak identification for as-cast Stellite 21. ............................. 78

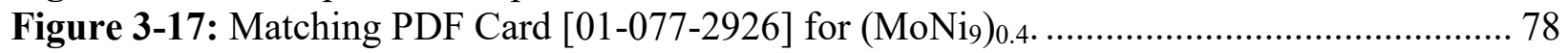

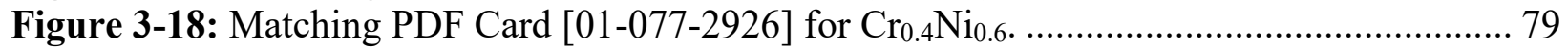

Figure 3-19: XRD pattern and peak identification for as-cast Stellite 720 ......................... 80

Figure 3-20: XRD pattern and peak identification for as-cast Stellite 728 . ........................... 81

Figure 3-21: Carbide volume fraction images of as-cast Stellite 21 (a) SEM image, (b)

thresholding image analysis ................................................................................... 82

Figure 3-22: Carbide volume fraction of as-cast Stellite 720 (a) SEM image, (b) thresholding image analysis of the large blocky carbide, (c) thresholding image analysis of the dendritic phase

Figure 3-23: Carbide volume fraction images of as-cast Stellite 728 (a) SEM image, (b) thresholding image analysis highlighting hard phases....................................................... 84

Figure 4-1: Types of break-in curve shapes, friction force versus time [18] ......................... 87

Figure 4-2: Pin-on-disc apparatus and computer control................................................... 88

Figure 4-3: Two types of specimens used for wear testing (a) stand alone specimen (Stellite

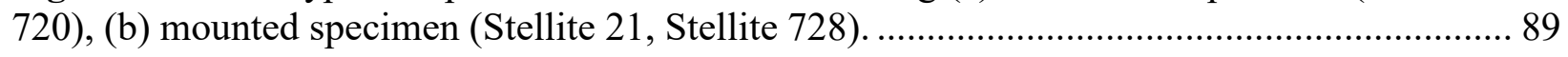

Figure 4-4: Pin-on-disc wear testing set-up (a) specimen in fixture, (b) overall structure of

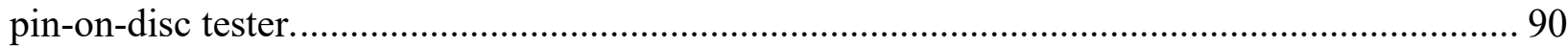

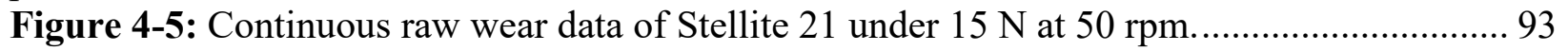


Figure 4-6: D150 Surface Profile Measuring System.

Figure 4-7: Wear tracks of Stellite 21 under $50 \mathrm{rpm}$ continuous testing (a) $15 \mathrm{~N}$ for $10 \mathrm{hrs}$, (b) $15 \mathrm{~N}$ for $20 \mathrm{hrs}$, (c) $15 \mathrm{~N}$ for $40 \mathrm{hrs}$, (d) $25 \mathrm{~N}$ for $10 \mathrm{hrs}$, (e) $25 \mathrm{~N}$ for $20 \mathrm{hrs}$, (f) $25 \mathrm{~N}$ for $40 \mathrm{hrs}$.

Figure 4-8: Wear tracks of Stellite 720 under $50 \mathrm{rpm}$ continuous testing at (a) $15 \mathrm{~N}$ for $10 \mathrm{hrs,}$ (b) $15 \mathrm{~N}$ for $20 \mathrm{hrs}$, (c) $15 \mathrm{~N}$ for $40 \mathrm{hrs}$, (d) $25 \mathrm{~N}$ for $10 \mathrm{hrs}$, (e) $25 \mathrm{~N}$ for $20 \mathrm{hrs}$, (f) $25 \mathrm{~N}$ for 40 hrs.

Figure 4-9: Wear tracks of Stellite 728 under $50 \mathrm{rpm}$ continuous testing at (a) $15 \mathrm{~N}$ for $10 \mathrm{hrs}$, (b) $15 \mathrm{~N}$ for $20 \mathrm{hrs}$, (c) $15 \mathrm{~N}$ for $40 \mathrm{hrs}$, (d) $25 \mathrm{~N}$ for $10 \mathrm{hrs,} \mathrm{(e)} 25 \mathrm{~N}$ for $20 \mathrm{hrs}$, (f) $25 \mathrm{~N}$ for 40 hrs.

Figure 4-10: Wear tracks for $50 \mathrm{rpm}$ interrupted testing under $25 \mathrm{~N}$ load for (a) Stellite 21 for time intervals up to $40 \mathrm{hrs}$, (b) Stellite 720 for time intervals up to $40 \mathrm{hrs}$, (c) Stellite 728 for time intervals up to $40 \mathrm{hrs}$. 96

Figure 4-11: Wear tracks for $60 \mathrm{rpm}$ interrupted testing under $25 \mathrm{~N}$ load for (a) Stellite 21 for time intervals up to $40 \mathrm{hrs}$, (b) Stellite 720 for time intervals up to $40 \mathrm{hrs}$, cracking due to hardness test overloading (c) Stellite 728 for time intervals up to $40 \mathrm{hrs}$............................... 96

Figure 4-12: Locations of four measurement points on wear track.................................... 97

Figure 4-13: An example of wear track cross-section profilometer measurement................... 97

Figure 4-14: Combined volume loss at $50 \mathrm{rpm}$ under $15 \mathrm{~N}$ and $25 \mathrm{~N}$ continuous testing. ........ 99

Figure 4-15: Volume loss for interrupted testing at $50 \mathrm{rpm}$ under 25 N. ............................ 102

Figure 4-16: Volume loss of continuous vs. interrupted testing under $25 \mathrm{~N}$ at $50 \mathrm{rpm} . \ldots \ldots \ldots . . .103$

Figure 4-17: Volume loss during interrupted testing at $50 \mathrm{rpm}$ and $60 \mathrm{rpm}$ under 25 N........ 104

Figure 4-18: Average friction coefficient for continuous testing at $50 \mathrm{rpm}$ under 15 N......... 105

Figure 4-19: Friction coefficient of continuous vs. interrupted testing at 50 rpm under 25 N.. 106

Figure 4-20: Friction coefficient for interrupted testing at $50 \mathrm{rpm}$ and $60 \mathrm{rpm}$ under 25 N.... 108

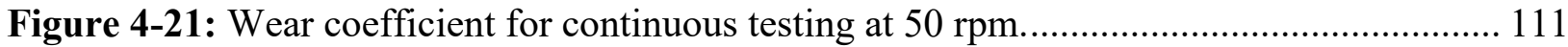

Figure 4-22: Combined interrupted and continuous wear coefficients at $50 \mathrm{rpm}$ under 25 N. . 112

Figure 4-23: Wear coefficient for interrupted testing at $50 \mathrm{rpm}$ and $60 \mathrm{rpm}$ under $25 \mathrm{~N}$....... 113

Figure 4-24: Worn surface of Stellite 21 continuously tested at $50 \mathrm{rpm}$ under $15 \mathrm{~N}$ for $10 \mathrm{hrs}$ $(0.94 \mathrm{~km})$ at (a) low magnification, (b) medium magnification, (c) high magnification. ......... 115

Figure 4-25: Worn surface of Stellite 21 continuously tested at $50 \mathrm{rpm}$ under $15 \mathrm{~N}$ for $20 \mathrm{hrs}$ $(1.88 \mathrm{~km})$ at (a) low magnification, (b) high magnification, (c) medium magnification. .......... 116

Figure 4-26: Worn surface of Stellite 21 continuously tested at $50 \mathrm{rpm}$ under $15 \mathrm{~N}$ for $40 \mathrm{hrs}$ $(3.77 \mathrm{~km}$ ) at (a) low magnification, (b) medium magnification, (c) high magnification. ......... 116

Figure 4-27: Worn surface of Stellite 21 continuously tested at $50 \mathrm{rpm}$ under $25 \mathrm{~N}$ for $10 \mathrm{hrs}$ (0.94 km) at (a) low magnification, (b) medium magnification, (c) high magnification. ......... 117

Figure 4-28: Worn surface of Stellite 21 continuously tested at $50 \mathrm{rpm}$ under $25 \mathrm{~N}$ for $20 \mathrm{hrs}$ $(1.88 \mathrm{~km})$ at (a) low magnification, (b) medium magnification.

Figure 4-29: Worn surface of Stellite 21 continuously tested at $50 \mathrm{rpm}$ under $25 \mathrm{~N}$ for $40 \mathrm{hrs}$ $(3.77 \mathrm{~km})$ at (a) medium magnification, (b) high magnification. ...................................... 118 Figure 4-30: Worn surface of Stellite 21 after interrupted test at $50 \mathrm{rpm}$ under $25 \mathrm{~N}$ for $40 \mathrm{hrs}$ $(3.77 \mathrm{~km})$ at (a) low magnification, (b) medium magnification.

Figure 4-31: Worn surface of Stellite 21 after interrupted test at $60 \mathrm{rpm}$ under $25 \mathrm{~N}$ for $40 \mathrm{hrs}$ $(3.77 \mathrm{~km}$ ) at (a) low magnification, (b) medium magnification.

Figure 4-32: Worn surface of Stellite 720 continuously tested at $50 \mathrm{rpm}$ under $15 \mathrm{~N}$ for $10 \mathrm{hrs}$ $(0.94 \mathrm{~km})$ at (a) low magnification, (b) medium magnification, (c) high magnification. 121 
Figure 4-33: Worn surface of Stellite 720 continuously tested at $50 \mathrm{rpm}$ under $15 \mathrm{~N}$ for $20 \mathrm{hrs}$ $(1.88 \mathrm{~km}$ ) at (a) low magnification, (b) high magnification, (c) medium magnification.

Figure 4-34: Worn surface of Stellite 720 continuously tested at $50 \mathrm{rpm}$ under $15 \mathrm{~N}$ for $40 \mathrm{hrs}$ $(3.77 \mathrm{~km})$ at (a) low magnification, (b) medium magnification, (c) high magnification. 122

Figure 4-35: Worn surface of Stellite 720 continuously tested at $50 \mathrm{rpm}$ under $25 \mathrm{~N}$ for $10 \mathrm{hrs}$ $(0.94 \mathrm{~km})$ at (a) low magnification, (b) medium magnification.

Figure 4-36: Worn surface of Stellite 720 continuously tested at $50 \mathrm{rpm}$ under $25 \mathrm{~N}$ for $20 \mathrm{hrs}$ $(1.88 \mathrm{~km})$ at (a) low magnification, (b) medium magnification. 123

Figure 4-37: Worn surface of Stellite 720 continuously tested at $50 \mathrm{rpm}$ under $25 \mathrm{~N}$ for $40 \mathrm{hrs}$ $(3.77 \mathrm{~km}$ ) at (a) low magnification, (b) high magnification, (c) medium magnification. ......... 124 Figure 4-38: Worn surface of Stellite 720 after interrupted test at $50 \mathrm{rpm}$ under $25 \mathrm{~N}$ for $40 \mathrm{hrs}$ $(3.77 \mathrm{~km}$ ) at (a) low magnification, (b) medium magnification, (c) high magnification. .......... 125 Figure 4-39: Worn surface of Stellite 720 after interrupted test at $60 \mathrm{rpm}$ under $25 \mathrm{~N}$ for $40 \mathrm{hrs}$ $(3.77 \mathrm{~km})$ at (a) low magnification, (b) high magnification. 125

Figure 4-40: Worn surface of Stellite 728 continuously tested at $50 \mathrm{rpm}$ under $15 \mathrm{~N}$ for $10 \mathrm{hrs}$ (0.94 km) at (a) low magnification, (b) medium magnification, (c) high magnification. 127

Figure 4-41: Worn surface of Stellite 728 continuously tested at $50 \mathrm{rpm}$ under $15 \mathrm{~N}$ for $20 \mathrm{hrs}$ $(1.88 \mathrm{~km}$ ) at (a) low magnification, (b) high magnification, (c) medium magnification. 127

Figure 4-42: Worn surface of Stellite 728 continuously tested at $50 \mathrm{rpm}$ under $15 \mathrm{~N}$ for $40 \mathrm{hrs}$ $(3.77 \mathrm{~km}$ ) at (a) low magnification, (b) high magnification, (c) medium magnification. .......... 128 Figure 4-43: Worn surface of Stellite 728 continuously tested at $50 \mathrm{rpm}$ under $25 \mathrm{~N}$ for $10 \mathrm{hrs}$ $(0.94 \mathrm{~km})$ (a) BSE image of a local area, (b) SE image of the same location. 129

Figure 4-44: Worn surface of Stellite 728 continuously tested at $50 \mathrm{rpm}$ under $25 \mathrm{~N}$ for $20 \mathrm{hrs}$ $(1.88 \mathrm{~km})$. (a) low magnification, (b) medium magnification, (c) high magnification. 130

Figure 4-45: Worn surface of Stellite 728 continuously tested at $50 \mathrm{rpm}$ under $25 \mathrm{~N}$ for $40 \mathrm{hrs}$ $(3.77 \mathrm{~km})$ at (a) medium magnification, (b) high magnification. 130

Figure 4-46: Worn surface of Stellite 728 after interrupted test at $50 \mathrm{rpm}$ under $25 \mathrm{~N}$ for $40 \mathrm{hrs}$ $(3.77 \mathrm{~km}$ ) at (a) low magnification, (b) medium magnification, (c) high magnification. 131

Figure 4-47: Worn surface of Stellite 728 after interrupted test at $60 \mathrm{rpm}$ under $25 \mathrm{~N}$ for $40 \mathrm{hrs}$ $(3.77 \mathrm{~km})$ at (a) low magnification, (b) medium magnification, (c) low magnification............ 131

Figure 4-48: Cracking in carbide structure of Stellite 728 worn surface............................... 132 Figure 4-49: Stellite 21 wear debris at 25 N 60 rpm for (a) 1 hour, (b) 60 hours duration...... 134 Figure 4-50: Stellite 720 wear debris at 25 N 60 rpm for (a) 1 hour, (b) 60 hours duration..... 135 Figure 4-51: Stellite 728 wear debris at 25 N 60 rpm for (a) 1 hour, (b) 60 hours duration.... 135 Figure 4-52: Wear debris for (a) Stellite 21 20hours, (b) Stellite 728 60hours duration.......... 136

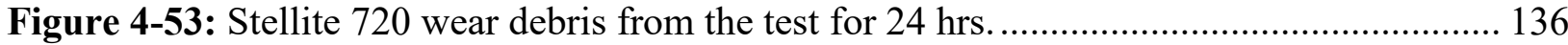
Figure 4-54: Debris EDX spectrum for Stellite 72060 rpm 25 N 60 hrs (a) analysis site, (b)

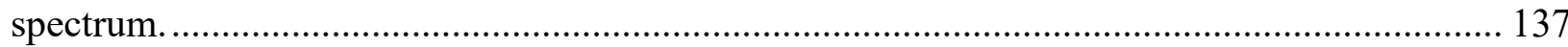

Figure 4-55: Oxygen content versus distance............................................................. 138 Figure 4-56: Particle size analysis of Stellite 21 wear debris from the test at 60 rpm under $25 \mathrm{~N}$.

Figure 4-57: Modeling results for continuous testing at $50 \mathrm{rpm}$ (a) under $15 \mathrm{~N}$, (b) under $25 \mathrm{~N}$.

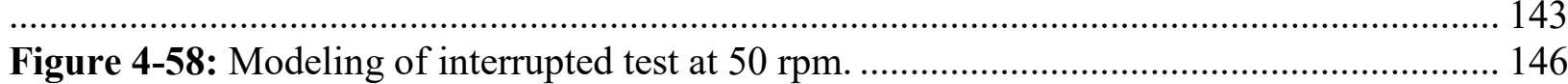

Figure 4-59: Modeling of interrupted test at $60 \mathrm{rpm}$.................................................. 147 
Figure 4-60: $C 1$ value vs. hardness (a) Continuous testing at $50 \mathrm{rpm}$ under $15 \mathrm{~N}$ and $25 \mathrm{~N}$, (b)

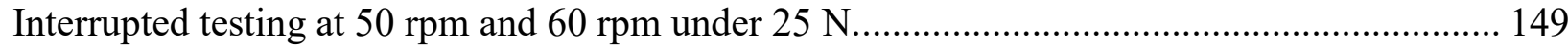




\section{Acronyms}

$\begin{array}{ll}\text { SEM } & \text { Scanning Electron Microscope/Microscopy } \\ \text { EDX } & \text { Energy-Dispersive X-ray } \\ \text { XRD } & \text { X-ray Diffraction } \\ \text { HV } & \text { Hardness Vickers Scale } \\ \text { HRC } & \text { Hardness Rockwell C Scale } \\ \text { HIP } & \text { Hot Isostatically Pressed } \\ \text { GIWM } & \text { Global Incremental Wear Model } \\ \text { FCC } & \text { Face Centred Cubic } \\ \text { HCP } & \text { Hexagonal Close Packed } \\ \text { DIC } & \text { Differential Interference Contrast } \\ \text { 3D } & \text { Three Dimensional } \\ \text { SEs } & \text { Secondary Electrons } \\ \text { BSEs } & \text { Backscatter Electrons } \\ \text { PDF } & \text { Powder Diffraction File } \\ \text { TEM } & \text { Transmission Electron Microscopy } \\ \text { ASTM } & \text { American Society for Testing of Materials }\end{array}$




\section{Nomenclature}

$\begin{array}{ll}\boldsymbol{P}_{\boldsymbol{u s d}} & \text { normal load per unit sliding distance } \\ \boldsymbol{p}_{\boldsymbol{m}} & \text { yield pressure (flow stress) } \\ \boldsymbol{H} & \text { hardness } \\ \boldsymbol{a} & \text { contact dimension i.e radius of wear particle } \\ \boldsymbol{V}_{\boldsymbol{p}} & \text { volume of a unit particle } \\ \text { K } & \text { proportion of wear particles generated under loading } \\ \boldsymbol{V} & \text { volume of material worn away per unit sliding distance } \\ \boldsymbol{w} & \text { worn volume per unit sliding distance } \\ \boldsymbol{P} & \text { normal load } \\ \boldsymbol{K}, \boldsymbol{\beta} & \text { wear coefficient } \\ \boldsymbol{s}, \boldsymbol{L} & \text { sliding distance } \\ \boldsymbol{W} & \text { worn volume/volumetric wear } \\ \boldsymbol{V}_{\boldsymbol{c}} & \text { worn volume composite } \\ \boldsymbol{k} & \text { dimensional wear coefficient } \\ \boldsymbol{\mu} & \text { friction coefficient } \\ \boldsymbol{h}^{\boldsymbol{w}} & \text { wear depth } \\ \boldsymbol{V}_{\boldsymbol{s}} & \text { volumetric wear of steel } \\ \boldsymbol{d} & \text { average particle diameter } \\ \boldsymbol{f}_{\boldsymbol{v}} & \text { particle volume fraction } \\ \boldsymbol{k}_{\mathbf{1}} & \text { proportional constant } \\ \boldsymbol{H}_{\boldsymbol{s}} & \text { hardness of the steel } \\ \boldsymbol{g}_{\boldsymbol{1}}, \boldsymbol{g}_{\mathbf{3}} & \text { constants } \\ \boldsymbol{C}_{\boldsymbol{1}} & \text { constant for total volume loss over the total distance } \\ \boldsymbol{C}_{\mathbf{2}} & \text { constant representing transition point from transient to steady-state wear } \\ \boldsymbol{K}_{\mathbf{1}} & \text { linear wear loss in the steady-state wear zone } \\ & \end{array}$




\section{Introduction}

\subsection{Background and Significance}

\subsubsection{Overview of Stellite Alloys}

Stellite is the name given to a family of cobalt-based alloys that are designed for use at high temperatures, in corrosive environments and wear applications. The alloys display a variety of wear resistance in both lubricated and unlubricated applications, and they also display excellent retention of hardness at relatively high temperatures. Their use spans a variety of industries from the oil and gas sector, to the medical industry.

The main alloying elements in Stellite alloys are carbon (C), chromium (Cr), tungsten (W) and/or molybdenum (Mo) with the balance of cobalt (Co). The balance of these elements in the alloy will provide a variety of properties targeted to meet the needs for particular applications. Carbon is a key element in the design of a Stellite alloy: the higher carbon alloys ( $\sim 1.2 \mathrm{wt} . \%$ to 3 wt.\%) are designed for wear resistance, the medium ( $\sim 0.5 \mathrm{wt} . \%$ to $1.2 \mathrm{wt} . \%)$ carbon alloys can be

for both wear and corrosion, and the low carbon alloys $(<0.5$ wt.\%) are designed more for corrosion, but also applicable to some wear applications. These ranges are a basic guide to understanding the alloys, and many alloys fall close to these ranges but can be used in a variety of applications [1].

The contributing factors to the properties of cobalt alloys are the crystallographic nature of cobalt, the formation of carbides, the corrosion resistance of chromium, and the solid-solutionstrengthening effects of alloying elements such as chromium, tungsten, and molybdenum [1]. The varying carbon content is related to the volume fraction of carbides that are able to form during solidification, the quantity of which will control the resulting properties of the alloy. 
Stellite alloys owe their properties to two strengthening mechanisms: solid-solution strengthening of the matrix, and precipitation of carbides [1]. The manufacturing processes associated with Stellite alloys can greatly affect the final microstructure, and different material properties can ensue [1]. Stellite alloys can be processed in several different ways: powder metallurgy, investment casting, hardfacing by welding, and sand casting, and each process affects the morphology of the carbides. In some instances, certain Stellite alloys are unable to be investment cast or sand cast due to the restraints imposed upon the cast shape by the investment mould. Post-processing can also prove to be quite difficult due to the fact that these alloys have been used historically as tools themselves, and are not easily machined to the required dimensions.

Stellite alloys contain many various alloying elements, and one feature is the ability to switch the tungsten wt.\% content for molybdenum. This switch still provides solid solution strengthening, but also improves corrosion in reducing environments [2]. The increase in molybdenum also changes the type of carbide formation. Three such alloys that utilize a change from tungsten to molybdenum are: Stellite 21 , Stellite 720 , and Stellite 728 . These alloys have varying degrees of both carbon and molybdenum. Stellite 21 being the lowest alloyed of the three with $0.25 \mathrm{wt} . \% \mathrm{C}$ and $5.5 \mathrm{wt} . \% \mathrm{Mo}$, has minimal carbides in the microstructure. Stellite 720 being the highest alloyed with $2.5 \mathrm{wt} . \% \mathrm{C}$ and $18 \mathrm{wt} . \% \mathrm{Mo}$, has a microstructure that consists of various types of blocky and fine carbides [3]. Stellite 728, stands somewhere in between these two with 0.3 wt.\% C and 12 wt.\% Mo, and formation of a eutectic network of carbides. However, the addition of niobium to this alloy, also assists with carbide formation [4]. So it can be appreciated that there are many varieties of alloying elements that will produce very different microstructures, and as a result, the final material properties will also change. 


\subsubsection{Properties and Applications}

One of the fundamental properties of Stellite alloys is their ability to be used in wear applications. Wear occurs in many different forms, and as is the case of metals, wear can be described by the deformation or removal of material from a surface. Most commonly, wear is evident by the presence of debris, i.e. detachment of particles from the surface, and markings on the surfaces where the wear has occurred. Types of wear include: sliding wear (adhesive wear), abrasive wear, erosive wear, and fretting wear [5]. The attribute of Stellite alloys having high yield strength and high work hardening rates is believed to prevent damage during sliding wear.

Corrosion resistance is another attribute Stellite alloys offer, and this is due to the high chromium content $(\sim 30 \mathrm{wt} \%)$ and the availability of low carbon Stellite alloys. Corrosion in single phase alloys can be considerably less due to the reduced number of interfaces between phases. Stellite alloys containing lower amounts of carbon have reduced carbide precipitates, and therefore fewer interfaces between phases, making them very useful in corrosive environments.

The varying elemental combinations of $\mathrm{Co}, \mathrm{C}, \mathrm{Cr}, \mathrm{W}$, and Mo give Stellite the versatility to be used in a variety of industries. From oil and gas drilling operations where the environment can be both abrasive and corrosive, to aerospace applications where the temperature in the engine is of critical importance and failure is not an option. Stellite alloys have the properties to lend themselves to some of the most difficult tasks industry has to offer. 


\subsection{Present Research}

\subsubsection{Objectives}

The development of Stellite alloys during their long history has shown that no one alloy has all the properties to combat the combined effort of wear, high temperature and corrosion. The Stellite family of alloys is extensive with many combinations of alloying elements being employed to meet particular applications. With such an extensive portfolio of alloys, and the possibility of different environments, the data can be somewhat limited. Modeling and wear maps exist for a few of the more commonly used Stellite alloys which contain a large amount of $\mathrm{W}$ as an alloying element. However, for the more recent Stellite alloys where Mo is employed instead of W, not a significant amount of data exists, particularly in the wear modeling field of research. Wear mechanisms are very complicated, and the relationship between the wear loss and imposing wear conditions is not always linear and easy to interpret. A greater understanding would be beneficial to document the wear of such Mo Stellite alloys, and investigate if their behaviour could be predicted in some way, and relate these findings to the microstructure of the alloy. The objectives of this present research are to:

(1) investigate the microstructure of three molybdenum Stellite alloys, designated as Stellite 21, Stellite 720 and Stellite 728, which contain different levels of Mo and C;

(2) model the behaviour of these alloys and validate this with test data via a pin-on-disc tribometer;

(3) enable wear prediction of other Stellite alloys to reduce the need for actual testing;

(4) evaluate the effect of microstructure on the wear properties of molybdenum Stellite alloys. 


\subsubsection{Tasks and Methodologies}

The selected molybdenum Stellite alloys, Stellite 21, Stellite 720 and Stellite 728, were investment cast and are from the molybdenum Stellite family where the usual W content of Stellite alloys has been substituted for molybdenum. The main feature of these alloys is the $\mathrm{C}$ content, but in the case of the Mo alloys, changes in the Mo and other element additions will affect the carbide formation, and hence the wear properties.

The microstructures were analyzed using a scanning electron microscope (SEM). To support the findings from the SEM, both Energy-Dispersive X-ray (EDX), and X-ray Diffraction (XRD) were employed to attempt to identify the type of carbides and intermetallic phases present.

A wear testing programme was carried out to gather data to validate the accuracy of Archard's wear equation with respect to the chosen Stellite alloys. The wear experiments were conducted at room temperature in dry-sliding mode using a pin-on-disc tribometer. Friction coefficient and wear loss data was collected during testing with the control software which monitored the experiment in real time. The actual wear loss was calculated based on a four-point measurement around the circumference of the wear track. The measurements were taken using a D150 Surface Profile Measuring System. The wear track was also evaluated using SEM and EDX to assess the condition of the surface post wear testing.

Based on experiments and data collection of the different microstructures, the following topics were discussed:

(a) Long-term wear testing on selected Stellite alloys

(b) Microstructural study on the selected Stellite alloys before and after wear testing

(c) Wear loss quantification

(d) Wear modeling using Archard's equation 
(e) Evaluation of the wear debris

\subsubsection{Organization of Thesis}

A comprehensive report of the present research is presented in this thesis. It consists of five chapters to introduce the topic, present the literature review findings, discuss the microstructural analysis, wear testing and modeling predictions, and finally the conclusions drawn from this research. A chapter overview is presented below:

Chapter 1 is an introduction to the present research. In this chapter, a brief overview of the properties and applications of Stellite alloys is presented; the significance, objectives, tasks, and methodologies of the present research are described.

Chapter 2 is the literature review which discusses Stellite alloys in more detail concerning their metallurgical and wear properties. General information on wear mechanisms and wear modeling is also presented with the present research.

Chapter 3 contains information on the microstructural analysis performed on the alloys used in the present research. Data and images obtained from SEM, EDX and XRD analysis, which is then used to describe the different microstructural constituents of the Stellite alloys are detailed.

Chapter 4 is the description of the wear testing evaluation using both modeling and actual test data. Procedural details to perform the ball-on-disc wear test are detailed, which include the apparatus, test parameters, operating procedure and wear loss calculation using a surface profile measuring system. Optical microscopy and SEM/EDX image analysis of the worn surfaces are presented, along with modeling predictions and actual test data. A brief evaluation of the wear debris generated from wear testing is also presented. Discussion of the wear data and predictions. 
This chapter will analyze the correlations between the data and discuss how microstructure affected the wear loss behaviour.

Chapter 5 contains the conclusions and future work. This is the summary chapter and presents conclusions drawn from the present research. Proposals for future work are presented with a viewpoint to building a knowledge base on the molybdenum Stellite alloys and how the microstructure affects the wear properties. 


\section{Literature Review}

\subsection{Synopsis of Stellite Alloys}

Stellite alloys are a group of cobalt-based alloys that were originally formulated from the investigations of Elwood Haynes in the early 1900s. The Stellite name is derived from the Latin word for 'star', stella, after the star like lustre exhibited by the alloys [6]. The Stellite alloys primarily consist of carbides within a solid solution matrix, and have properties that lend themselves to heat, wear, and corrosion applications. Cobalt has both metallurgical and non-metallurgical uses, and when used unalloyed, it is relatively soft (HV140 - 250 or $\sim$ HRC22) [1]. Metallurgically, when alloyed with elements such as chromium, carbon, molybdenum, and tungsten, cobalt alloys become very hard, in excess of hardness HRC40 for some alloys.

The primary use of cobalt-based alloys is to manufacture wear-resistant components for demanding applications such as those associated with the oil and gas industrial sectors. Chromium $(\mathrm{Cr})$, tungsten (W) and, molybdenum (Mo) alloying elements provide the metal carbides for wear resistance, and chromium, in particular, has the dual functions of also providing corrosion resistance. Stellite alloys have evolved from being used originally as cutting tool materials, to having an important role in a variety of industrial sectors.

\subsection{Physical Metallurgy of Stellite Alloys}

The properties of Stellite alloys are governed by the size, shape, type and volume fraction of carbides that form within the matrix. Along with the carbon content, both the processing of the alloy and subtle chemistry changes strongly influence carbide formation, which consequently affect the performance of the alloy in different working environments. A list of common Stellite alloys is presented in Table 2-1. 
Table 2-1: Chemical compositions (wt.\%) of common Stellite alloys [2]

\begin{tabular}{|c|c|c|c|c|c|c|c|c|c|c|}
\hline Alloy & Process & $\mathrm{Cr}$ & $\mathrm{W}$ & $\mathrm{Mo}$ & $\mathrm{C}$ & $\mathrm{Fe}$ & $\mathrm{Ni}$ & $\mathrm{Si}$ & $\mathrm{Mn}$ & Others \\
\hline Stellite 1 & Cast & 30 & 13 & 0.5 & 2.5 & 3 & 1.5 & 1.3 & 0.5 & \\
\hline Stellite 3 & P/M & 30.5 & 12.5 & & 2.4 & 5 & 3.5 & 2 & 2 & $1 \mathrm{~B}$ \\
\hline Stellite 4 & Cast & 30 & 14 & 1 & 0.57 & 3 & 3 & 2 & 1 & \\
\hline Stellite 6 & Cast & 29 & 4.5 & 1.5 & 1.2 & 3 & 3 & 1.5 & 1 & \\
\hline Stellite 6 & P/M & 28.5 & 4.5 & 1.5 & 1 & 5 & 3 & 2 & 2 & $1 \mathrm{~B}$ \\
\hline Stellite 12 & Cast & 30 & 8.3 & & 1.4 & 3 & 1.5 & 0.7 & 2.5 & \\
\hline Stellite 21 & Cast & 27 & & 5.5 & 0.25 & 3 & 2.75 & 1 & 1 & \\
\hline Stellite 20 & Cast & 33 & 17.5 & & 2.45 & 2.5 & 2.5 & & 1 & \\
\hline Stellite 22 & Cast & 27 & & 11 & 0.25 & 3 & 2.75 & 1 & 1 & \\
\hline Stellite 25 & Cast & 20 & 15 & & 0.1 & 3 & 10 & 1 & 1.5 & \\
\hline Stellite 31 & Cast & 22 & 7.5 & & 0.5 & 1.5 & 10 & 0.5 & 0.5 & \\
\hline Stellite 80 & Cast & 33.5 & 19 & & 1.9 & & & & & $1 \mathrm{~B}$ \\
\hline Stellite 188 & Cast & 22 & 14 & & 0.1 & 3 & 22 & 0.35 & 1.25 & $0.03 \mathrm{La}$ \\
\hline Stellite 190 & Weld & 27 & 14 & 1 & 3.3 & 3 & 3 & 1 & 1 & \\
\hline Stellite 300 & Cast & 22 & 32 & & 1.5 & & & & & \\
\hline Stellite 694 & Cast & 28 & 19 & & 1 & 5 & & 1 & 1 & \\
\hline Stellite 712 & Cast & 29 & & 8.5 & 2 & 3 & 3 & 1.5 & 1.5 & \\
\hline Stellite 720 & Cast & 33 & & 18 & 2.5 & 3 & 3 & 1.5 & 1.5 & $0.3 \mathrm{~B}$ \\
\hline Stellite 703 & Cast & 32 & & 12 & 2.4 & 3 & 3 & 1.5 & 1.5 & \\
\hline Stellite 706 & Cast & 29 & & 5 & 1.2 & 3 & 3 & 1.5 & 1.5 & \\
\hline Stellite 706K & Wrought & 29 & & 6 & 1.4 & 3 & 3 & 1.5 & 1.5 & \\
\hline Stellite 728 & Cast & 24 & & 12 & 0.35 & 1 & 3.8 & 0.45 & 0.52 & $2 \mathrm{Nb}$ \\
\hline Stellite 6B & Wrought & 30 & 4 & 1.5 & 1 & 3 & 2.5 & 0.7 & 1.4 & \\
\hline Stellite 6K & Wrought & 30 & 4.5 & 1.5 & 1.6 & 3 & 3 & 2 & 2 & \\
\hline Stellite 98M2 & P/M & 30 & 18.5 & 0.8 & 2 & 5 & 3.5 & 1 & 1 & $4.2 \mathrm{~V}, 1 \mathrm{~B}$ \\
\hline
\end{tabular}


As a general guide, Stellite alloys contain 25 - 33 wt.\% Cr, 4 - 17 wt.\% W, and 0.1 - 3.3 wt.\% C. In some Stellite alloys, the role of W is exchanged for Mo. The microstructure of a Stellite alloy consists of a cobalt-chromium-tungsten/molybdenum (Co-Cr-W, Mo) solid solution (matrix) with one or more carbide phases. The solid solution usually contains small amounts of iron (Fe), nickel (Ni), silicon ( $\mathrm{Si}$ ) and manganese (Mn). The carbides are most commonly identified as $\mathrm{M}_{23} \mathrm{C}_{6}, \mathrm{M}_{7} \mathrm{C}_{3}$, and $\mathrm{M}_{6} \mathrm{C}$, where $\mathrm{C}$ is carbon, and $\mathrm{M}$ is the metal component [7]. The type of alloying elements and the processing route are significant in order to develop the material properties for each individual alloy.

\subsubsection{Carbon Content and Carbides}

The $\mathrm{Cr}$ and $\mathrm{W}$ contents in Stellite alloys provide solid solution strengthening to the matrix, and the carbides provide hardness and wear resistance. The principal carbide former is $\mathrm{Cr}$, with $\mathrm{W}$ also playing a role. The most notable trend of Stellite alloys is that the $\mathrm{W}$ content is usually increased with the $\mathrm{C}$ content. As $\mathrm{W}$ contributes to carbide formation, this ensures that a reasonable amount of $\mathrm{Cr}$ still remains in the matrix for corrosion resistance.

Stellite alloys can be classified in two different ways. Firstly, from a practical perspective, three main alloy groups have evolved based on the carbon content:

i) Low-carbon alloys having up to $0.5 \mathrm{wt} . \% \mathrm{C}$, such as Stellite 31 having $0.31 \mathrm{wt} . \% \mathrm{C}$, which is used in high-temperature applications.

ii) Medium-carbon alloys having 0.5 to $1.2 \mathrm{wt} \% \mathrm{C}$, such as Stellite 6 having $1.2 \mathrm{wt}$ \% C, which is used in dual applications of both moderate wear and corrosion.

iii) High-carbon alloys having 1.2 to 3.5 wt.\% C, e.g., Stellite 1 having 2.5 wt.\% C being used primarily for wear applications. 
Secondly, from a metallurgical perspective, Stellite alloys are considered to be either hypoeutectic (Figure 2-1) or hypereutectic (Figure 2-2). The alloys with very low C, $<0.5$ wt.\%, are considered to be solid solution strengthened.

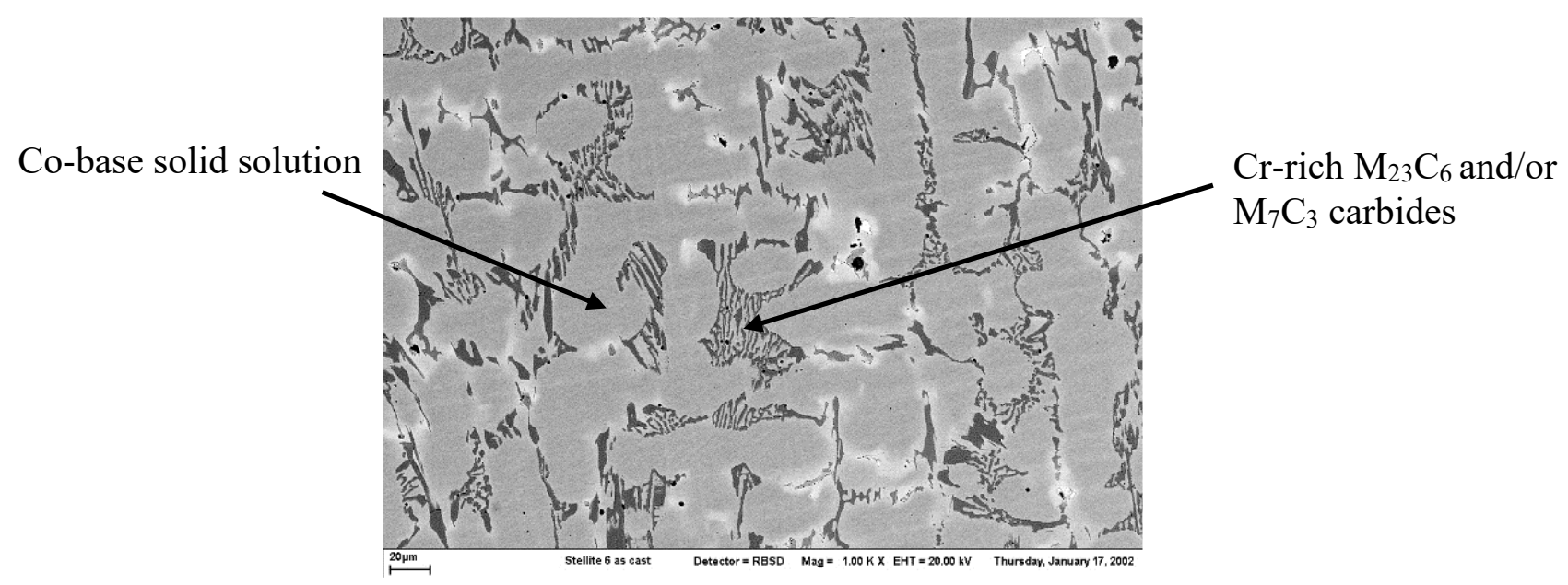

Figure 2-1: Stellite 6 cast microstructure (Figure courtesy of Kennametal Inc.).

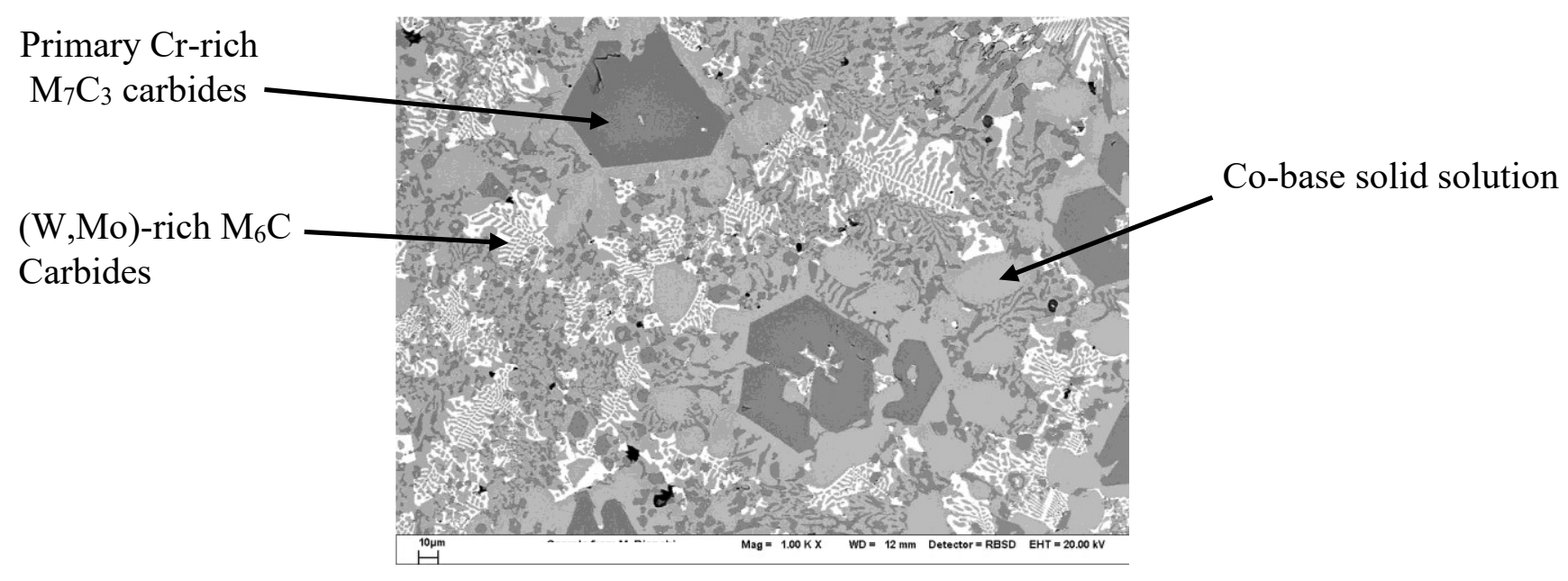

Figure 2-2: Stellite 20 cast microstructure (Figure courtesy of Kennametal Inc.).

Hypoeutectic Stellite alloys involve the formation of primary Co-Cr-(W, Mo) dendrites, and eutectic carbides precipitate, interdendritically [8]. The interdendritic region may also contain 
$\mathrm{CoCr}(\mathrm{W}, \mathrm{Mo})$ eutectic. In hypereutectic alloys, carbides are the primary phase. These hypereutectic alloys have large idiomorphic carbide phases, which appear blocky, spiny or rodlike [8]. After the carbide formation, a mixture of $\mathrm{CoCr}(\mathrm{W}, \mathrm{Mo})$ dendrites and a $\mathrm{CoCr}(\mathrm{W}, \mathrm{Mo})-$ $\mathrm{M}_{7} \mathrm{C}_{3} / \mathrm{M}_{6} \mathrm{C}$ lamellar eutectic forms [8]. The lamellar eutectic nucleates on and between the initial primary (idiomorphic) carbides [8]. This character of Stellite alloys can be represented by the pseudoternary $(\mathrm{C}, \mathrm{Co}, \mathrm{Cr}+\mathrm{W})$ system shown in Figure 2-3 where the hypoeutectic is designated as $L+\gamma$ to the left of the eutectic, and the hypereutectic phase is designated as $L+\mathrm{M}_{7} \mathrm{C}_{3}$ to the right of the eutectic [9]. The eutectic line is considered to be around $2 \mathrm{wt} \% \mathrm{C}[8]$, and is affected by a number of alloying elements. The hypoeutectic alloys are generally used for dual wear and corrosion applications such as that found in oil and gas downhole drilling where wet soils and abrasion particulates are present simultaneously. Hypereutetic alloys are commonly used for more wear applications such as sealing surfaces on engine valves.

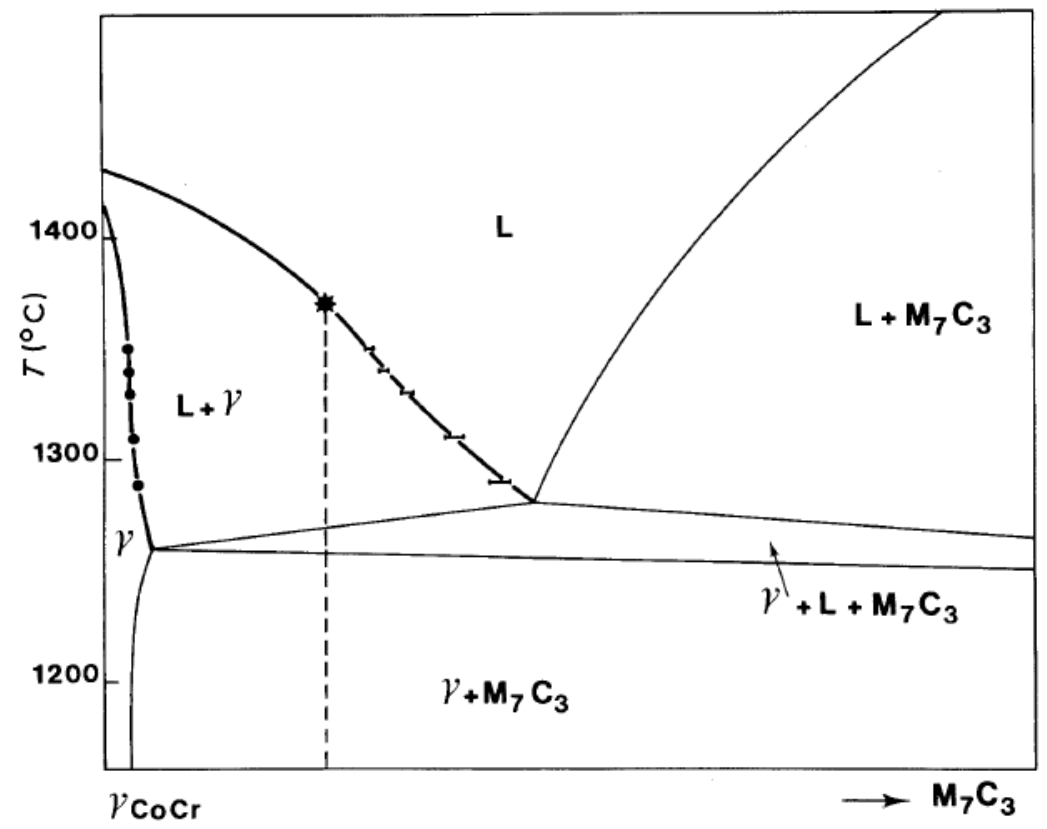

Figure 2-3: Vertical section of pseudoternary $(\mathrm{C}, \mathrm{Co}, \mathrm{Cr}+\mathrm{W})$ system. $\mathrm{L}$ is liquid, $\mathrm{M}$ represents metal components, $\gamma$ represents fcc phase [9]. 
The nominal carbon content ranges of Stellite alloys can be very interesting, in so much that a Stellite alloy having $\sim 2$ wt.\% C can be either hypoeutectic or hypereutectic when considering the allowable carbon range during the manufacturing process.

Generally, four types of carbides can be considered influential in cobalt-base alloys. They are $\mathrm{MC}, \mathrm{M}_{7} \mathrm{C}_{3}, \mathrm{M}_{23} \mathrm{C}_{6}$, and $\mathrm{M}_{6} \mathrm{C}$, where $\mathrm{M}$ represents one or more metallic elements [10]. In Stellite alloys, the predominant carbides are $\mathrm{M}_{7} \mathrm{C}_{3}, \mathrm{M}_{23} \mathrm{C}_{6}$ and $\mathrm{M}_{6} \mathrm{C}$ type. Due to the microstructural sensitivities, particularly with different carbon ranges, various carbides can be found in Stellite alloys, most commonly $\mathrm{Cr}$-rich $\mathrm{M}_{7} \mathrm{C}_{3}$, and $\mathrm{M}_{23} \mathrm{C}_{6}$, as well as (Co, W, Mo) ${ }_{6} \mathrm{C}$ [8]. However, other metals in the alloys can be partially substituted in place of the Cr, W, and Mo [8].

Carbides have two major roles in the microstructure of Stellite alloys. Firstly, the carbides form a network structure to resist plastic deformation; and, secondly, carbides provide strength through dispersion hardening. Certain elements have tendencies to form in particular carbide types. Chromium precipitates weakly in $\mathrm{M}_{6} \mathrm{C}$ and $\mathrm{MC}$ types, but forms strongly in $\mathrm{M}_{23} \mathrm{C}_{6}$ and $\mathrm{M}_{7} \mathrm{C}_{3}[10]$. This means that certain carbides are more likely to form depending on the elements present.

$\mathrm{M}_{23} \mathrm{C}_{6}$ (fcc) is a low-temperature form of the carbides and, depending on the alloy, generally goes into solution near $1900^{\circ} \mathrm{F}$ [10]. This type of carbide tends to form at grain boundaries, and can precipitate as film, globules, platelets, lamellae and a cellular growth [10]. Chromium is the principal metallic radical of $\mathrm{M}_{23} \mathrm{C}_{6}$, but depending on the quantity of alloying elements and the time at temperature, other metallic elements such as $\mathrm{Ni}, \mathrm{Co}, \mathrm{Fe}, \mathrm{Mo}$, and $\mathrm{W}$ can take the place of chromium to form the carbide. [10].

$\mathrm{M}_{6} \mathrm{C}$ (fcc) predominantly forms radicals with heavy elements such as Mo and $\mathrm{W}$, although some solubility with $\mathrm{Cr}$, $\mathrm{Ni}$ and $\mathrm{Co}$ can occur $[10] . \mathrm{M}_{6} \mathrm{C}$ is found in the as-cast microstructure and 
is stable at higher temperatures than $\mathrm{M}_{23} \mathrm{C}_{6}$ [10]. $\mathrm{M}_{6} \mathrm{C}$ precipitates as blocky carbides on the grain boundaries, and can form Widmanstätten structures [11].

For $\mathrm{M}_{7} \mathrm{C}_{3}$ (hexagonal), the predominant metallic radical is $\mathrm{Cr}$ with limited solubility for Ni. The phase is stable at higher temperatures than $\mathrm{M}_{23} \mathrm{C}_{6}$ [10]. As with $\mathrm{M}_{23} \mathrm{C}_{6}, \mathrm{M}_{7} \mathrm{C}_{3}$ forms at grain boundaries as irregular discontinuous, blocky particles, although plates and regular geometric forms have also been observed.

$\mathrm{MC}$ carbides have a cubic crystal structure and can take intergranular and transgranular positions, often interdendritically [11]. These carbides are the most stable compounds in nature. The preferred order of formation (in order of decreasing stability) in superalloys for these carbides is $\mathrm{HfC}, \mathrm{TaC}, \mathrm{NbC}$, and $\mathrm{TiC}[11]$.

\subsubsection{Effects of Processing on Microstructure}

Stellite alloys can be tailored for different applications by adjusting the chemical composition, and even more so when a different manufacturing method is used to process the same alloy. Stellite alloys can be manufactured and processed in many different methods: investment casting, sand casting, centrifugal casting, powder metallurgy, thermal spray, hardfacing by welding, etc. A change in the manufacturing process also results in changes to the microstructure, which in turn changes the material properties.

The formation of carbides in Stellite alloys depends not only on the composition but also the manufacturing process. The carbides impart brittleness to Stellite alloys, particularly for the cast microstructures which have coarse carbides. Therefore, by changing the manufacturing route, the carbides can be refined. Stellite 20 is a prime example of this degree of change. The cast structure shown in Figure 2-4 presents itself as rod-like primary Cr-rich carbides, lamellar W-rich 
carbides, and CoCrW matrix [12]. This same Stellite alloy, when processed using hot-isostatic pressing (HIP), a process of heating and pressuring consolidated powder in a sealed metallic container, yields a very different microstructure, as shown in Figure 2-5. This microstructure presents itself as one containing fine carbides uniformly distributed in the matrix [12]. However, although visually the alloys are very different, metallurgically the phases are very similar i.e., $\alpha$ cobalt, $\mathrm{M}_{7} \mathrm{C}_{3}, \mathrm{Cr}_{23} \mathrm{C}_{6}, \mathrm{Co}_{3} \mathrm{~W}, \mathrm{Co}_{7} \mathrm{~W}_{6}$ and $\mathrm{Co}_{6} \mathrm{~W}_{6} \mathrm{C}[12]$.

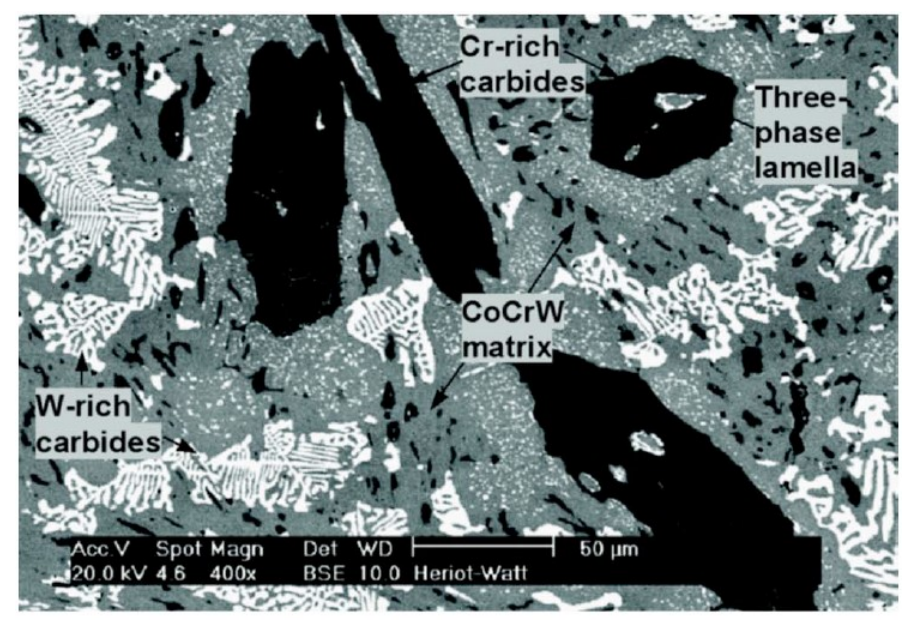

Figure 2-4: Stellite 20 cast microstructure [12].

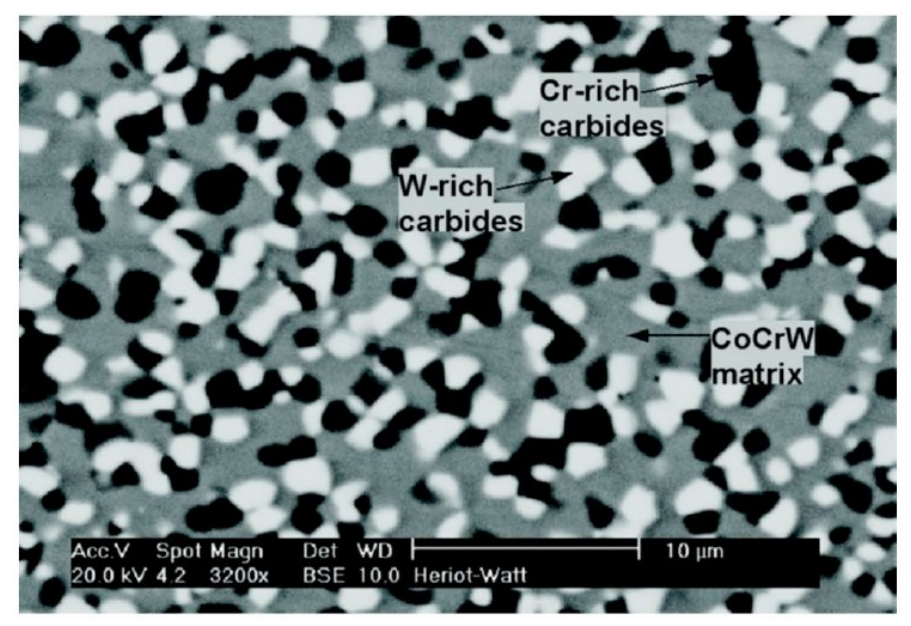

Figure 2-5: Stellite 20 HIPed microstructure [12]. 
The difference in material properties for this Stellite 20 example is mostly centred on the impact toughness and contact fatigue. The HIP'ed Stellite 20 alloy showed the impact toughness to be an order of magnitude higher than the cast Stellite 20, for which the fine carbide morphology is considered to be the governing factor [12].

\subsubsection{Molybdenum Effects}

Stellite alloys employ two major types of solid solution strengthening elements, namely W and Mo. In the originally formulated Stellite alloys, tungsten forms a large part of the chemical compositions. However, new alloys are being developed to meet new demanding applications, thus the numbering designation system for Stellite alloys has been quite varied during the history of the alloys. Almost exclusively, Stellite 21 being an exception, the 700 series Stellite alloys employ Mo in exchange for W. The exchange of Mo for W enables a greater degree of corrosion resistance in combating environments such as sulphuric acid [2]. Molybdenum also forms the carbides which enhance wear resistance, when present in a large quantity [2].

In common practice, the chemical composition is measured in terms of weight percent, and $\mathrm{W}$ is substituted with Mo with similar weight percentage. Mo having roughly half the atomic weight of $\mathrm{W}$ allows for a greater presence of Mo in the 700 series alloys. Therefore, for the equivalent weight percentage of $\mathrm{W}$, there are approximately twice as many Mo atoms. This will change the carbide morphology and increase the volume fraction of carbides in the microstructures $[3]$.

Stelilte 21 is a low C (0.2 wt.\%) Stellite alloy that employs Mo to strengthen the solid solution instead of $\mathrm{W}$. The low $\mathrm{C}$ content allows considerably more $\mathrm{Cr}$ and $\mathrm{Mo}$ to remain in the solid solution rather than in the $\mathrm{M}_{7} \mathrm{C}_{3}$ carbides, as shown in Figure 2-6 [2]. As previously 
discussed, this allows the alloy with more elements in solid solution to have a greater degree of corrosion resistance in certain environments.

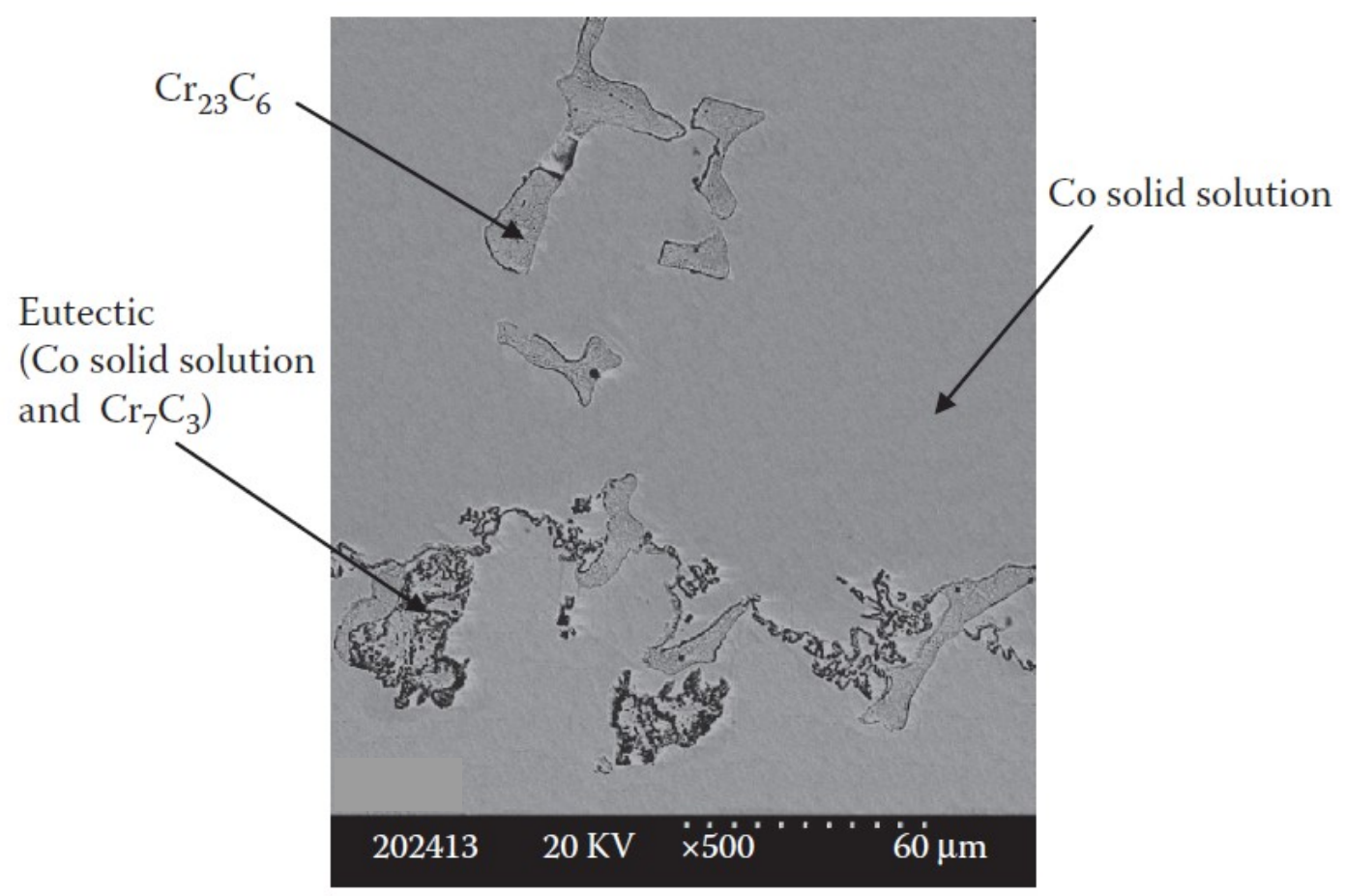

Figure 2-6: Stellite 21 cast microstructure [2].

Stellite 720 , which is the equivalent Mo counterpart of Stellite 20, produces a microstructure consisting of primary Cr-rich carbides and surrounding secondary carbide eutectic mixtures, as seen in Figure 2-7. The eutectic mixture consists of a $(\mathrm{Co}, \mathrm{Mo})_{3} \mathrm{C}$ carbide (white phase), and a $\mathrm{Cr}_{7} \mathrm{C}_{3}$ eutectic (light grey), as shown in Figure 2-8. The remainder is Co solidsolution, and the black phase is $\mathrm{Cr}_{7} \mathrm{C}_{3}[3]$. 


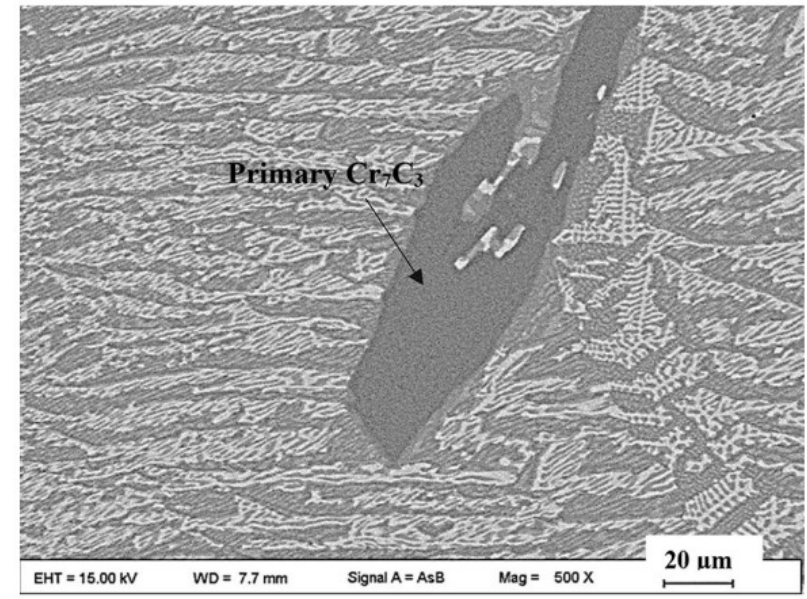

Figure 2-7: Stellite 720 cast microstructure [3].

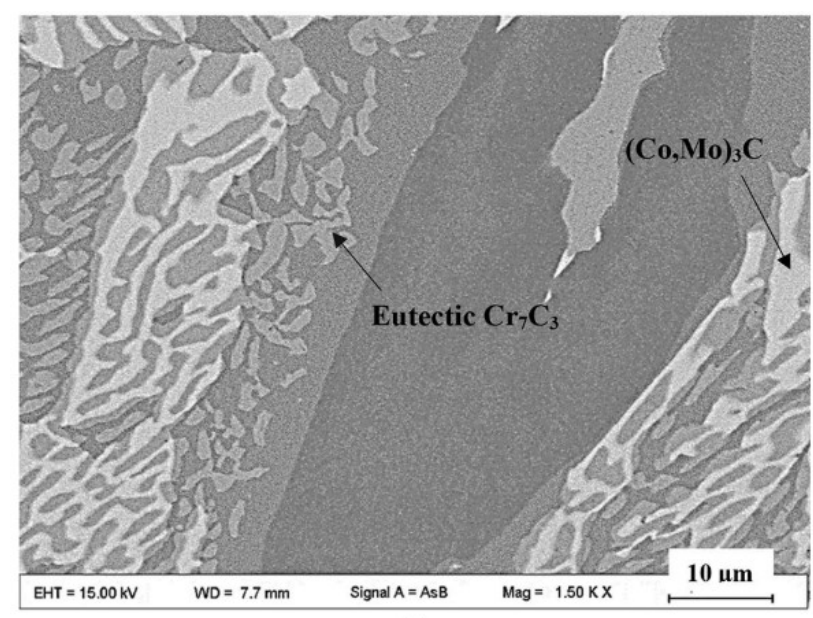

Figure 2-8: Stellite 720 cast microstructure at higher magnification [3].

Excessive carbides and intermetallics may form in the Co-Cr-Mo Stellite alloys due to the presence of C, $\mathrm{Cr}$ and Mo [4]. Therefore, the $\mathrm{C}$ content is kept to a certain level to allow the Mo and $\mathrm{Cr}$ additions to form solid solutions and impart improved corrosion resistance to the alloy. However, with the lower $\mathrm{C}$ content come fewer carbides, which means a lowering of hardness. This in turn negatively affects the wear resistance. Stellite 728 having a relatively high Mo content at 12 wt.\%, and low C at 0.35 wt.\% is a newer Stellite alloy. This alloy also employs a 2 wt.\% Nb content to tie up the $\mathrm{C}$ and leave enough $\mathrm{Cr}$ and $\mathrm{Mo}$ in solid solution to provide high-temperature 
wear and corrosion resistance [4]. Stellite 728 has a hypo-eutectic microstructure consisting of a eutectic network of CoCrMo solid solution (dendrites), and various fine lamellar carbides, as shown in Figure 2-9 [4]. This combination of elements has shown Stellite 728 to have improved abrasion and sliding wear resistance over Stellite 21 [4].

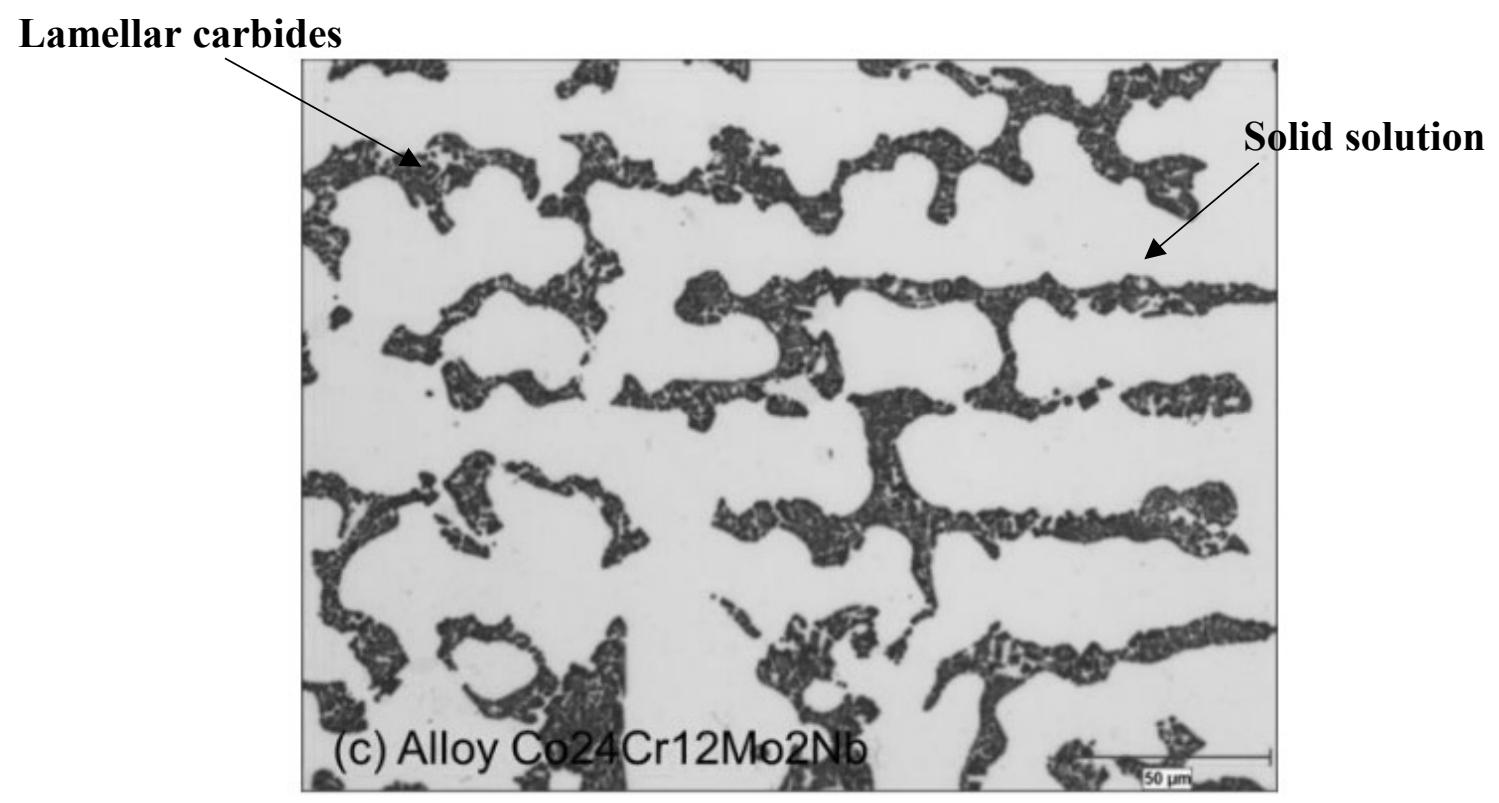

Figure 2-9: Stellite 728 cast microstructure [4].

\subsection{Types of Wear to Stellite Alloys}

Three main types of wear may be incurred by Stellite alloys; they are abrasive, sliding, and erosive wear [2]. Understanding the type of wear is important in choosing the correct alloy for a specific application. Various Stellite alloys have been developed for particular applications. The focus in industrial applications tends toward the component with the most potential for economic loss. As a general rule, wear loss is not inherent to the material but is dependent on many governing factors such as working environment, mating materials, surface preparation of components, time, 
stress levels etc. However, material properties such as hardness and microstructure can mostly affect the wear loss.

\subsubsection{Abrasive Wear}

\subsubsection{Wear Mechanism}

Abrasive wear can be described as the relative movement of hard projections (surface asperities), or hard particles (external or trapped wear debris), against a surface, as shown in Figure 2-10 [13]. The type of contact between the hard projections can be two-body or three-body. Two body contact means that two contact surfaces slide against each other, and three body contact involves an abrasive medium caught between the contacting surfaces, as shown in Figure 2-11 [13].

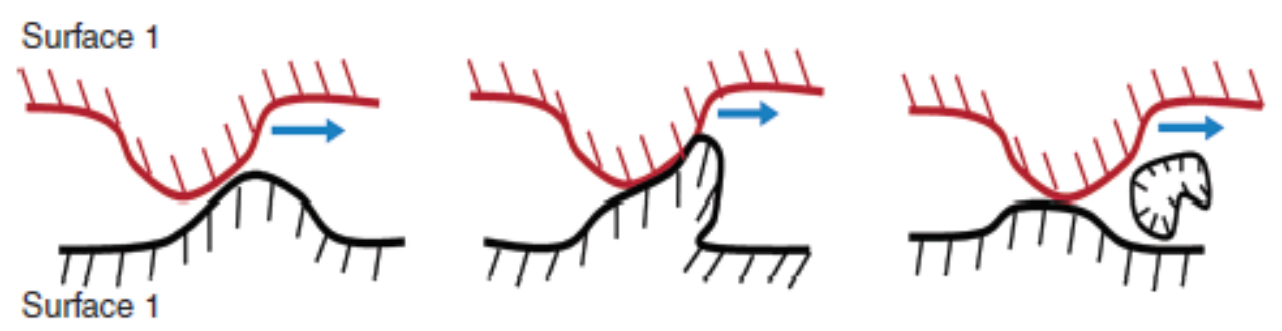

Figure 2-10: Illustration of abrasive wear mechanism [13].
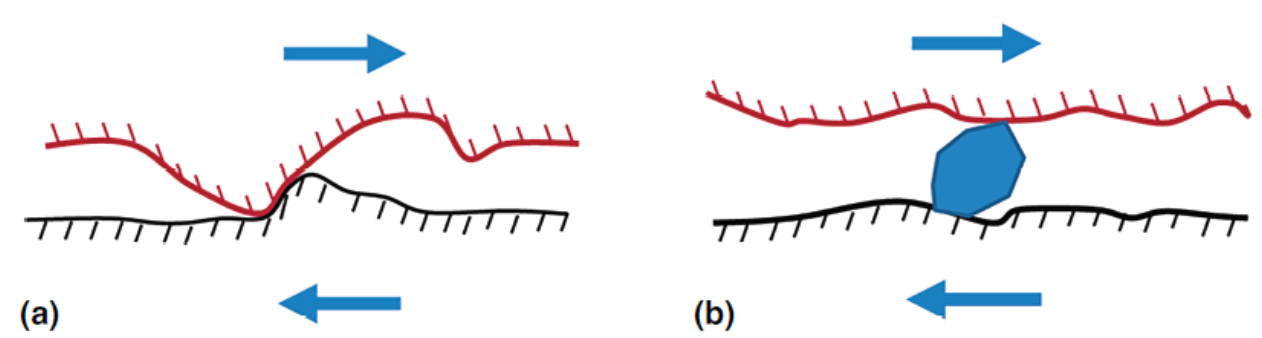

(b)

Figure 2-11: Two types of abrasive wear: (a) two-body abrasion, (b) three-body abrasion [13]. 
The abrasive medium (particles or projections) can be used to categorize abrasion further by indicating if high or low-stress abrasion is occurring. High stress means that the abrasive medium is crushed, and low stress means that the abrasive medium is relatively intact. In both cases, the contact stress can cause varying degrees of wear [13].

The physical processes associated with abrasion wear are shown in Figure 2-12, which consist of microploughing, microcutting, microfatigue and microcracking [14]. Different mechanisms will prevail depending on material properties and the surrounding environment. The material properties include hardness, while alloying elements are necessary to develop the microstructure constituents with specific crystalline structure. The external environmental factors include the abrasive(s) in the system (size, shape, type), temperature, the speed of contact, loading on the system, humidity and corrosive effects.

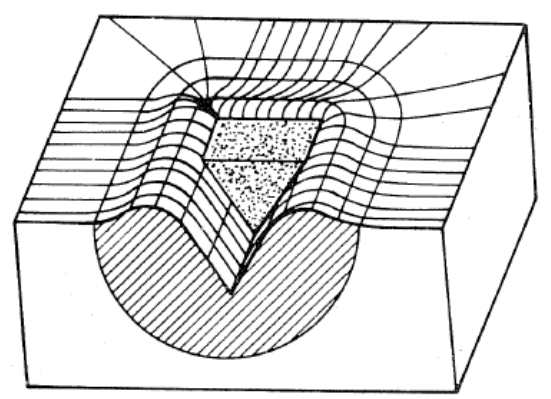

Microploughing

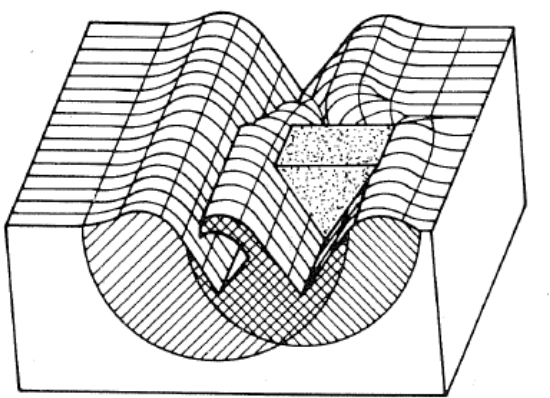

Microfatigue

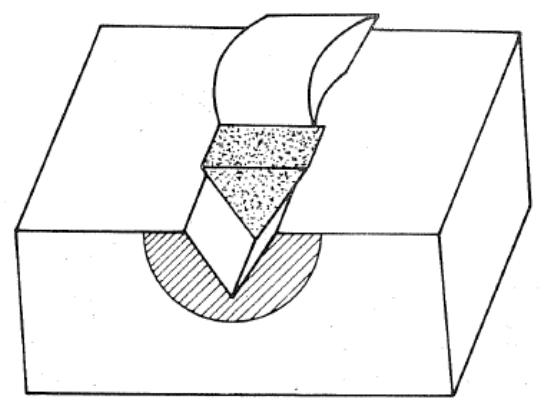

Microcutting

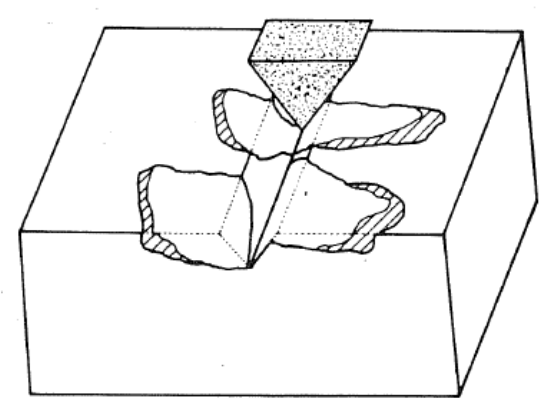

Microcracking

Figure 2-12: Abrasive wear physical processes [14]. 


\subsubsection{Effects of Material Properties}

Hardness has been shown to correlate closely with material's abrasion rate [14][7], but it should be considered as an indicator rather than a definitive relationship. The abrasion process induces plastic deformation at a certain degree, which can result in extensive work hardening of the material being abraded. For this reason, correlation of the wear data using the hardness of the as-prepared wear surface, as opposed to the worn work hardened surface, may not be representative [14]. In general, the hardness of a material in relation to abrasion resistance can be represented as shown in Figure 2-13 where transition points between mechanisms are identified [14].

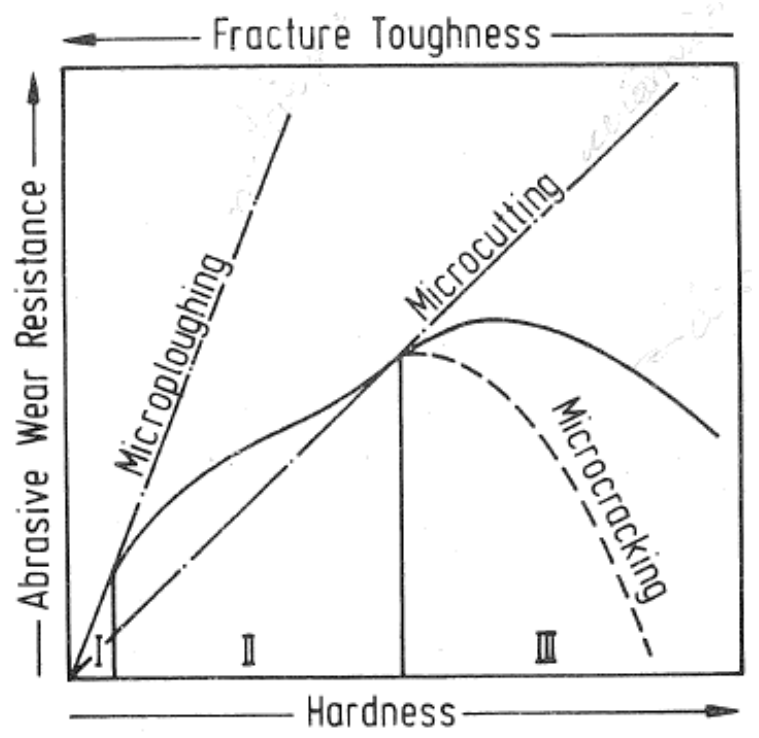

Figure 2-13: Abrasive wear versus hardness schematic [14].

One notable transition is from microcutting to microcracking, which is identifiable in harder materials in general. At this point the abrasion resistance of materials can be quickly deteriorated. 
Crystal structure and orientation have been found to influence the abrasion wear of a material. For example, cubic metals, wear at approximately twice the rate of hexagonal metals, which is attributed to the lower work hardening rate of the hexagonal metals [15]. The orientation of crystallographic planes in a prepared surface of single crystal ((001) plane of body centred cubic (bcc) and single crystal face centred cubic (fcc) influences the surface wear, showing a wider scratch width, i.e. higher wear, along the $<100>$ than the $<110>$ direction [16]. In the case of steels for example, microconstituents can generally be ranked from martensite (the highest), bainite, austenitic manganese steel, pearlite and finally ferrite (the lowest) [13]. However, depending on the heat treatment and composition the abrasion resistance of these phases can change. Fine pearlite for example, is more resistant to wear than coarse perlite [13].

Alloying, via interstitial or substitutional methods, has long been proven to increase the wear resistance of materials. A popular way to improve wear resistance is to generate a harder second phase. Precipitation can lead to a significant improvement in hardness and yield stress. Consideration must be paid to both the degree of coherency, i.e. lattice match, and the size of the precipitates. A general concept applicable to abrasion wear is that a mismatch i.e., difference in size, between the abrasion particles and the hard particles in the matrix are desirable. As a general rule, incoherent particles can be effective in decreasing the total wear when they are somewhat larger than the abrasive particles [7]. The carbides in Stellite alloys are incoherent, which enhance good abrasion resistance. The wear in the case of incoherent particles occurs by abrasion of the hard particles and debonding due to fatigue [7].

In the case of Stellite alloys, the hard carbide phase will protect the matrix from wear to some degree. However, the load will be eventually transferred to the carbide phase as the matrix 
is worn away. The higher loads on brittle materials, such as some grades of Stellite alloys, will cause cracking and chipping over an area larger than the abrasive particles causing the damage.

The size of the abrasive particles and the particle size of the harder phases are important. Incoherent particles that are relatively smaller than the abrasive particles will be removed during the wear on the matrix. However, having larger incoherent particles is not necessarily a viable option if the distance between the harder incoherent phases is larger than the abrasive particle size. In this instance, the abrasive medium can wear the material between the harder phases causing the harder phase to be exposed and fall out [7].

In general, carbides are removed quite aggressively from the microstructure via pulling, digging, cracking and cutting. The abrasive particles may also experience some wear, which in some cases will be rounding of sharp edges. A hard, tough and blocky phase works considerably well for wear protection [7]. The higher hardness makes them difficult to cut and having a level of toughness reduces the risk of brittle fracture during loading. Plate- or rod-shaped harder phases are more likely to propagate cracks and fracture as opposed to the blocky particles [7]. Figure 2-14 shows the interactions of different sized carbides with both hard and soft abrasive particles. Small carbides are easily removed (Figure 2-14a) by hard abrasive particles, and large brittle carbides may be cut and cracked (Figure 2-14c). For instances where softer abrasive particles are present, small carbides can still be dug out of the material $1 \mathrm{~d}$ ), or large pits can form in the matrix around the carbides from the action of the soft abrasive (Figure 2-14e).

Silence et al. [13] reported findings of the abrasive wear of Co- and Fe-base alloys and how this type of wear was affected by carbide morphology. Figure 2-15 shows the results from the dry silica rubber wheel test with various samples of different carbide morphologies. The different morphologies were obtained by casting in both graphite (faster cooling rate yielding a fine carbide 
structure), and sand (slower cooling rate yielding a coarser carbide structure). The results show that a coarser carbide morphology resulted in up to 10 times greater wear resistance than the finer carbide morphology [14][17]. Another important noteworthy finding was that even though the sand cast alloys were a couple of Rockwell Hardness points below the graphite cast alloys, their abrasion resistance was higher [14][17].

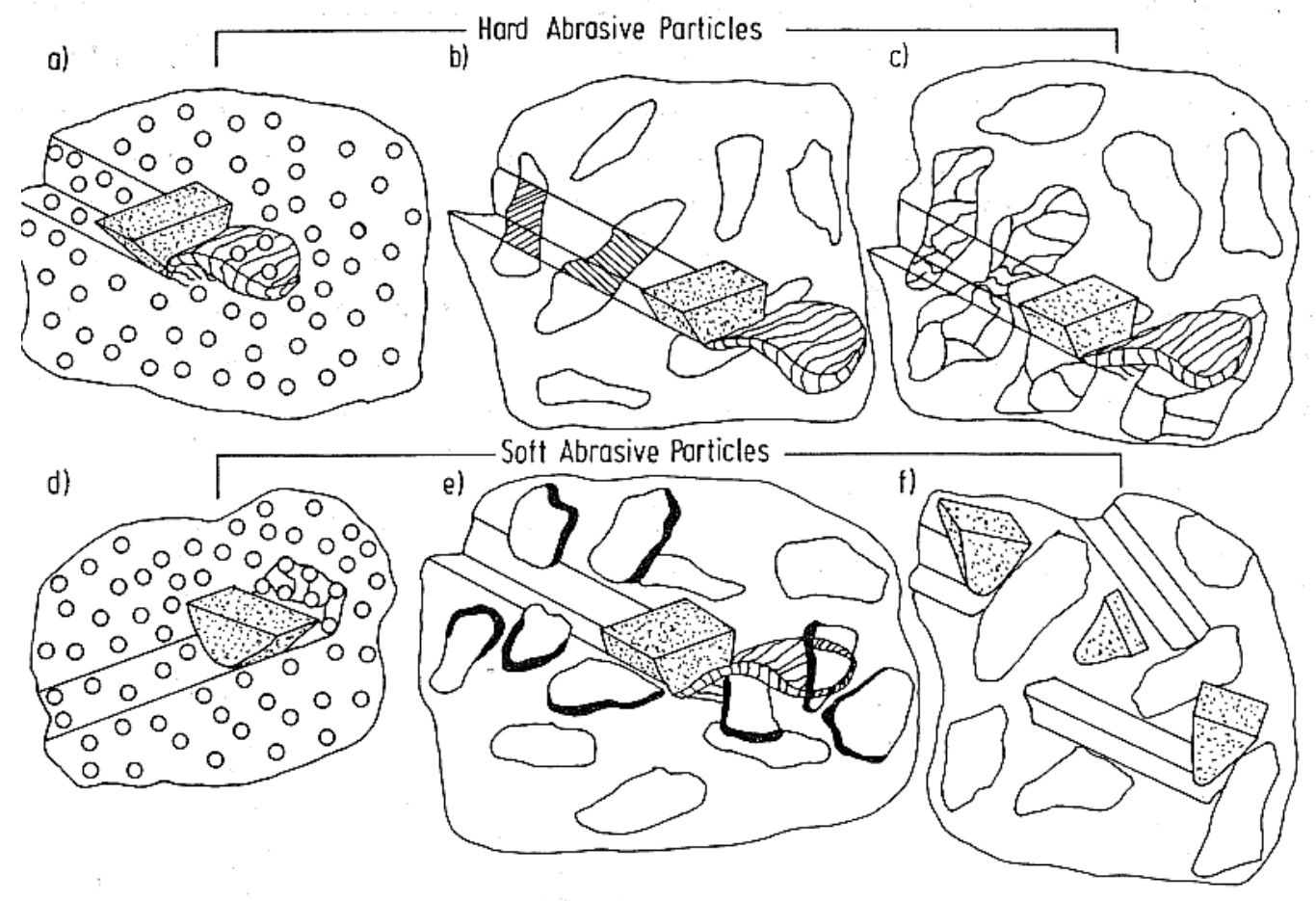

Figure 2-14: Interactions of sliding hard or soft abrasive particles and carbides [13]. 


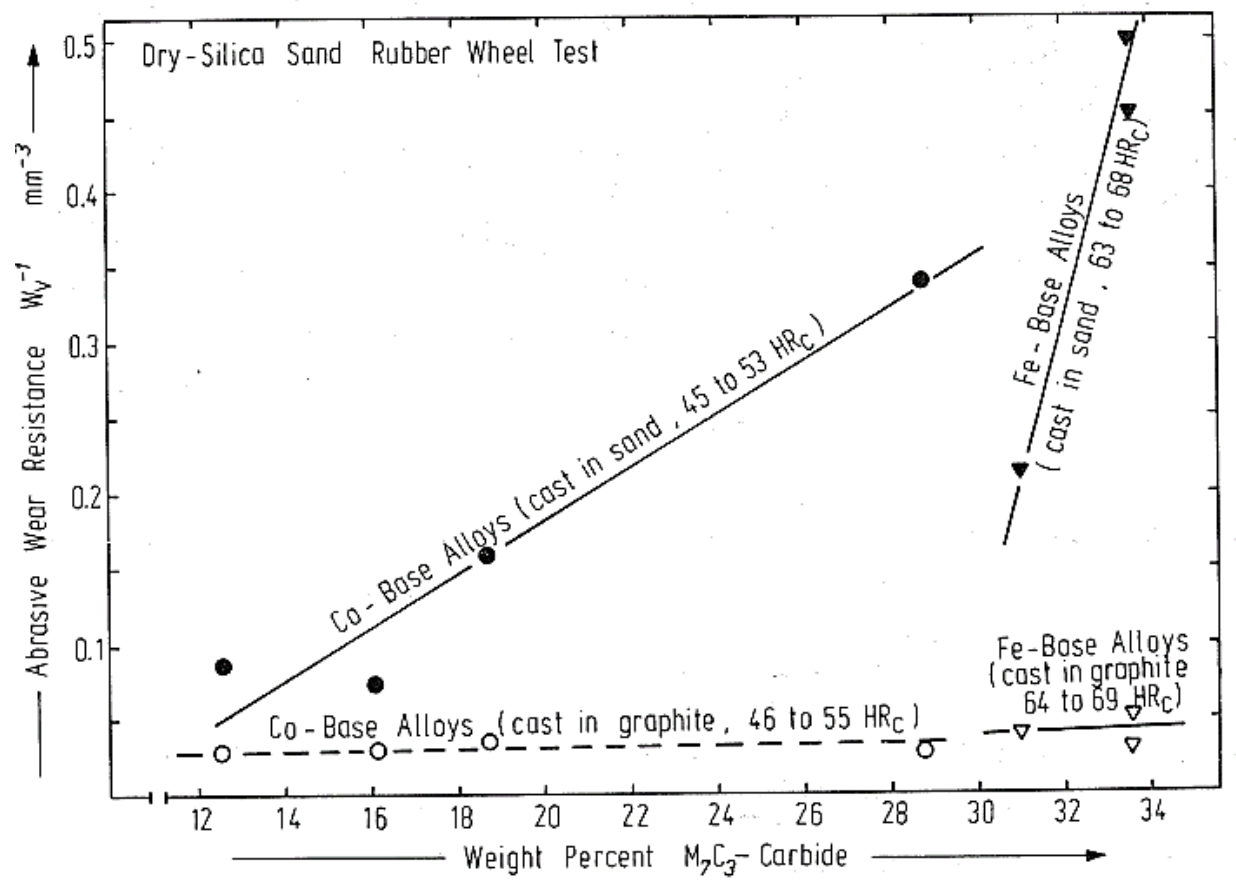

Figure 2-15: Abrasive wear versus wt.\% of $\mathrm{M}_{7} \mathrm{C}_{3}$ carbides for sand and graphite cast Co- and Fe-based alloys [13].

\subsubsection{Environmental Effects}

The characteristics of the abrasive medium, such as hardness, toughness, and size, all contribute to the overall wear rate. If the hardness of the abrasive exceeds that of the wear material, there is a greater tendency for the abrasive to attack and dig into the surface without much effect on the abrasive itself; the abrasive can retain its characteristics e.g. sharp edges that can cut into the wear material. The shape of the abrasive medium affects how it attacks and removes the wear material. A more rounded shape will cause less wear than sharp particles due to a lower stress concentration at the contacting faces, and an abrasive with high toughness will increase wear loss [7]. Temperature also plays a major role in abrasive wear. In general, materials lose mechanical strength at high temperatures, which will lower their wear resistance [13]. 
Understanding the environment influence on wear can provide valuable insight on what material to choose for suitable application. The surrounding environment can affect the abrasion mechanism. Corrosion may be accelerated by the damage induced by the abrasive medium, and vice-versa, corrosion acting on the surface enables particles to be removed more easily by the abrasive medium [13].

\subsubsection{Sliding and Adhesion Wear}

Sliding wear and adhesive wear are the two common terms for the wear mechanism where a solid body is sliding along the surface of another solid body under a nonzero normal force [13]. Although both terms describe the same event of wear occurring, sliding denotes the type of relative motion, and adhesion implies a mechanism as to how the wear occurs [13]. The terminology increases in complexity when considering that sliding wear incorporates other wear processes such as abrasion and gouging.

The adhesion mechanism can be described as local junctions forming from contacting asperities due to the plastic deformation of the surface materials under high local pressure. These

asperities are under sliding motion during the wearing process, thus the junctions rupture and material will transfer from one surface to another [14]. Adhesion wear is most identifiable when normally moving components seize and can no longer function correctly e.g. a seized shaft inside a bearing. An example of a surface worn by sliding wear is shown in Figure 2-16. 


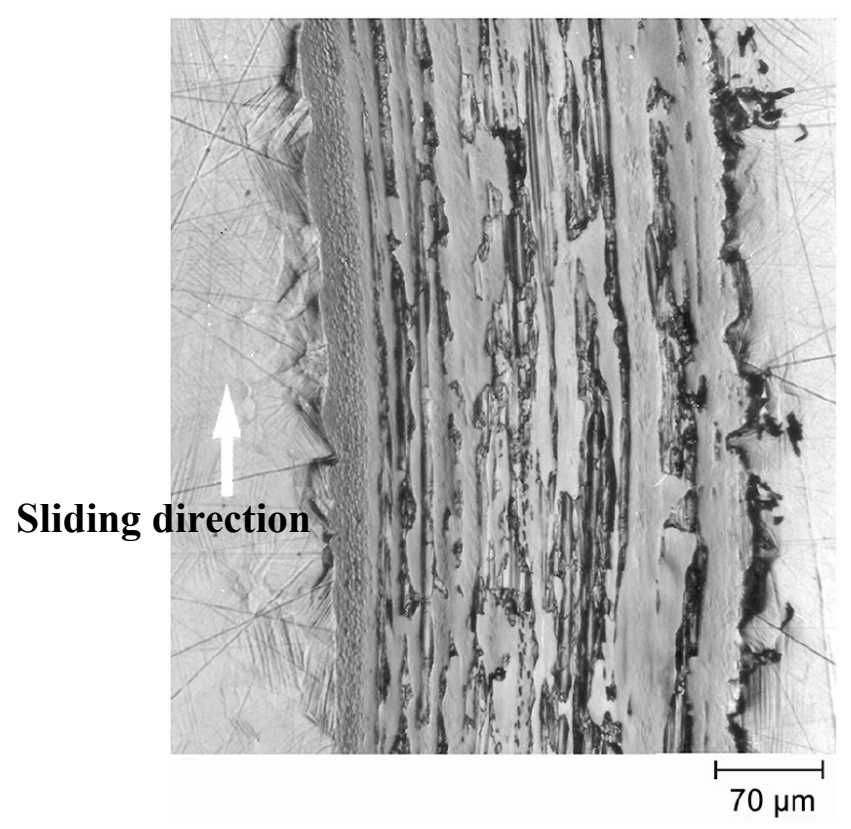

Figure 2-16: Severely worn surface of a $\mathrm{Cu}-10 \mathrm{wt} \% \mathrm{Zn}$ alloy disc when sliding against an AISI 52100 steel ball in argon gas. [13].

Other wear processes previously mentioned can occur in sliding wear systems in a variety of ways, one of which is by debris from the asperities becoming entrapped and acting as an abrasive or gouging the material.

The relative motion of sliding wear can occur in different ways, most commonly these are linear oscillation, unidirectional sliding, torsional oscillation and multidirectional sliding, as shown in Figure 2-17 [13]. 

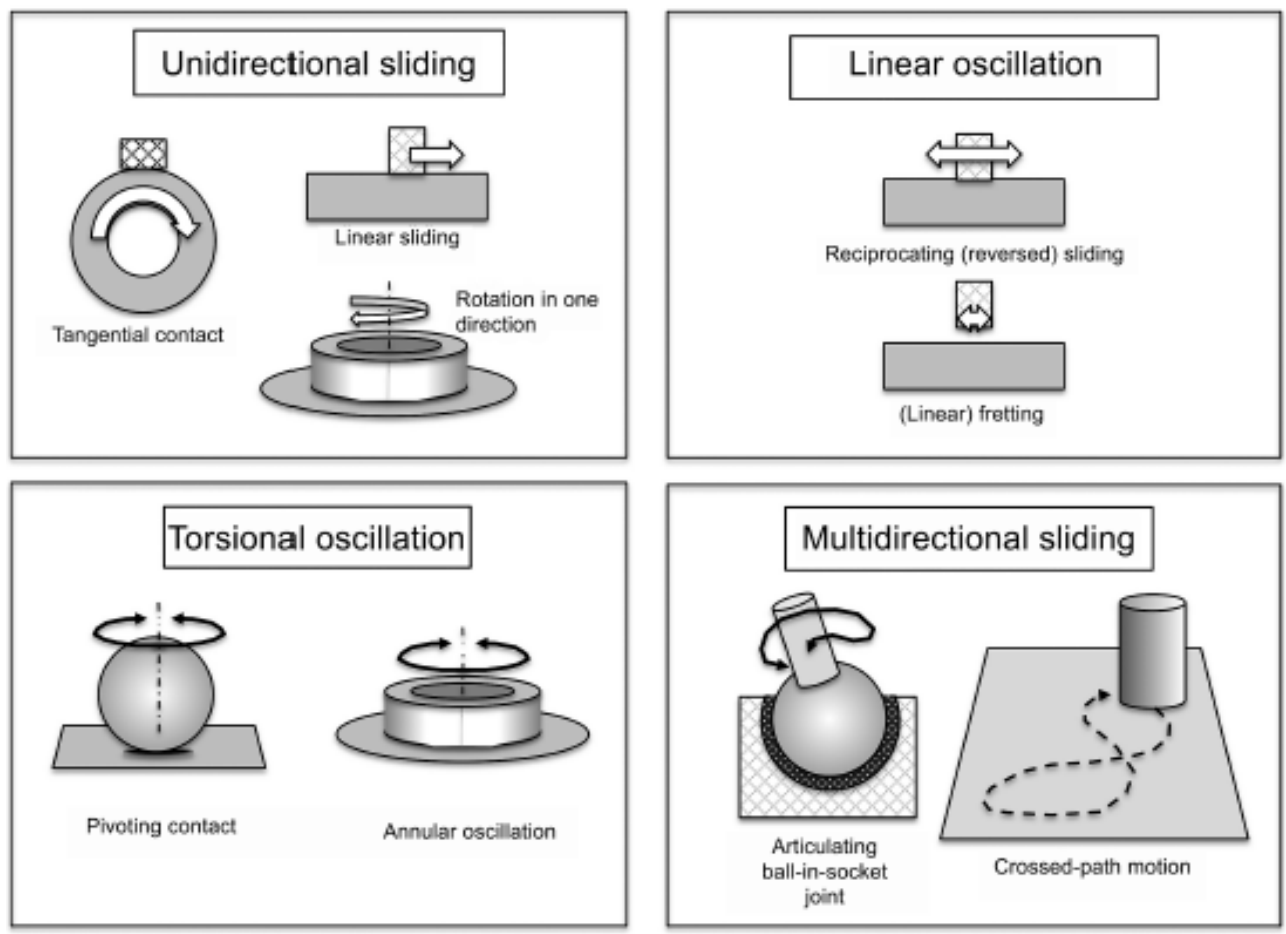

Figure 2-17: Various modes of relative sliding motion and contact geometry [13].

Fretting wear can be accommodated in a few sliding wear categories e.g. linear oscillation, if the relative motion is a few or hundreds of micrometers [13]. The direction of motion can affect a lot of different aspects of siding wear e.g. the movement of wear particles. It is, therefore, preferable to simulate the type of motion if this type of wear is being investigated for a particular application. 


\subsubsection{Influential Factors}

Sliding wear progresses in three stages: Stage I, the running-in period (sometimes referred to as the transient period), followed by Stage II, the steady-state period, and III, the breakdown period, as depicted in Figure 2-18 [14].

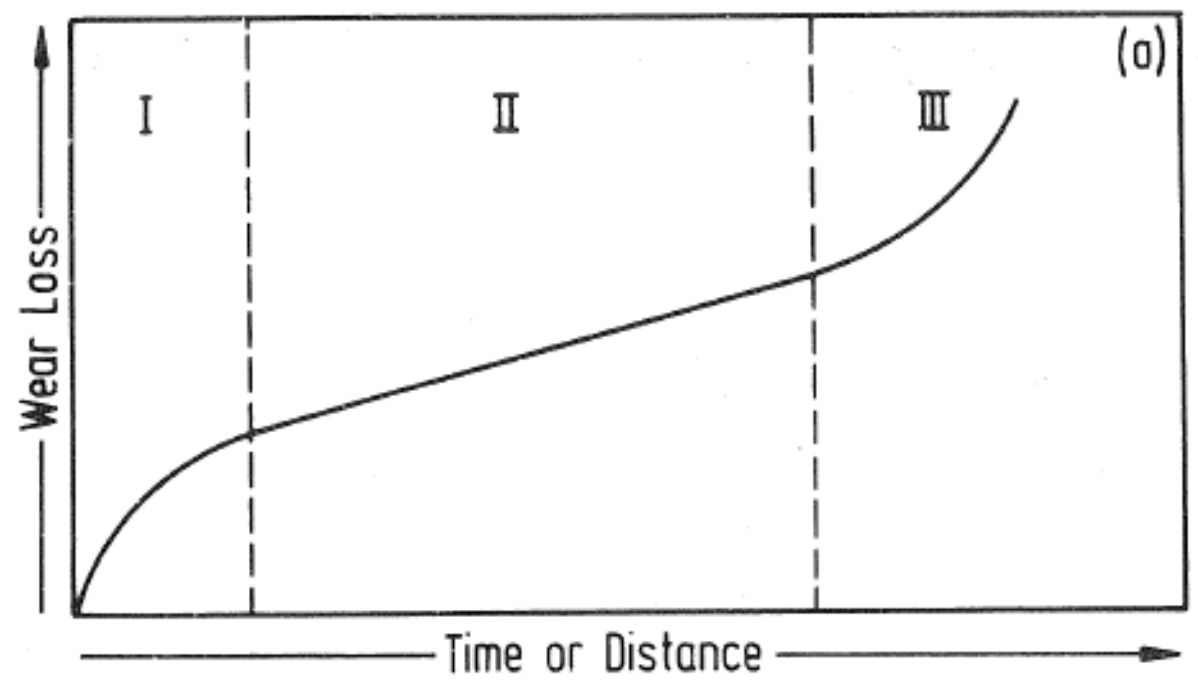

Figure 2-18: Schematic plot of wear loss as a function of time or sliding distance (general progress) [14].

The running-in period, also termed breaking-in or wear-in, occurs at the beginning of a wear process where the wear rate fluctuates, along with friction and temperature changes of the fresh, unworn solid surfaces [18]. As the contact area increases, a steady state ensues. Adhesion, surface fatigue, abrasion and/or tribochemical reactions can occur in a sliding wear system, and the wear loss for each process will present itself in a different way (Figure 2-19) [14]. 


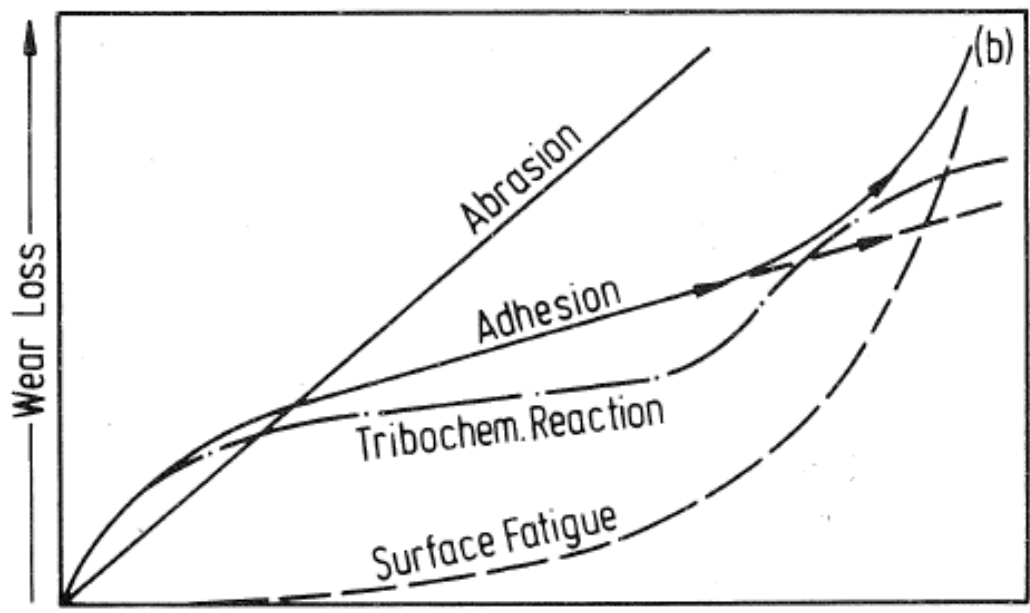

Time or Distance

Figure 2-19: Wear loss as a function of time or sliding distance showing the different features of the four wear sliding wear mechanisms [14].

Different interactions will affect the resulting wear mechanism (Figure 2-20). The common feature is elastic or plastic deformation, which is affected by tangential traction, contact area and material properties. The type of deformation is significant, but also the features of the counterbody, the loading conditions etc. all play a part in determining the wear mechanism [14].

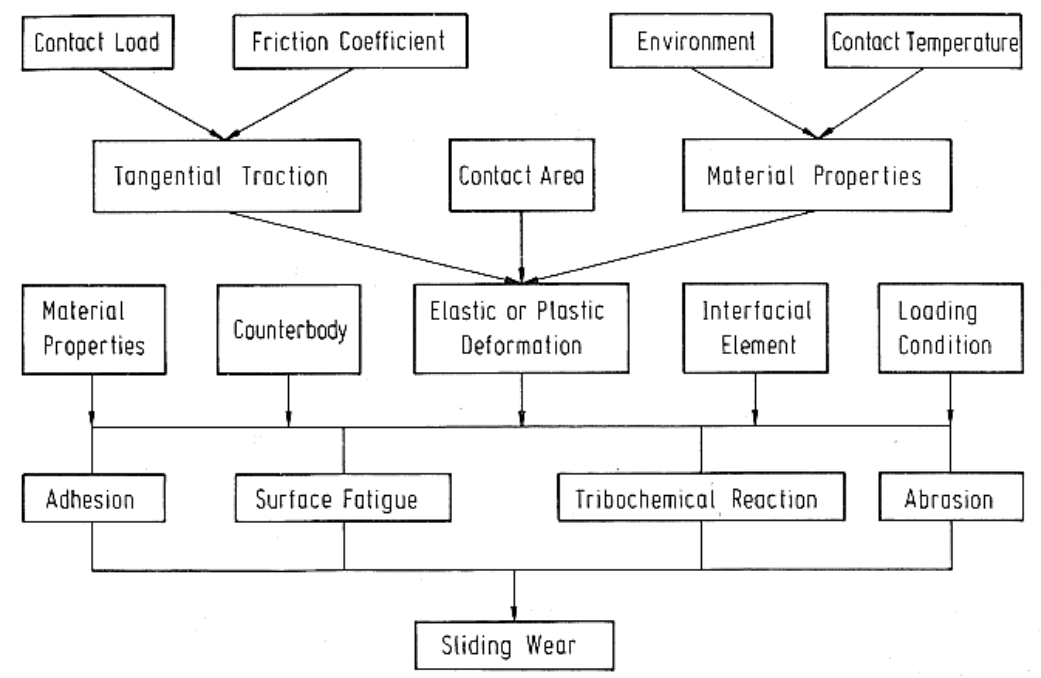

Figure 2-20: Factors influencing wear mechanisms during sliding contact [14]. 
The adhesion junctions formed during sliding wear are governed by many physical and chemical properties of the materials in the system. In a lot of instances, the contact between metals is non-metallic due to the surfaces having oxide films and adsorbed layers [14]. This feature of sliding wear is important for Stellite alloys in that the high chromium content will lend itself to forming protective surface oxides [14][8]. Figure 2-21 shows the range of metallurgical properties influencing sliding wear.

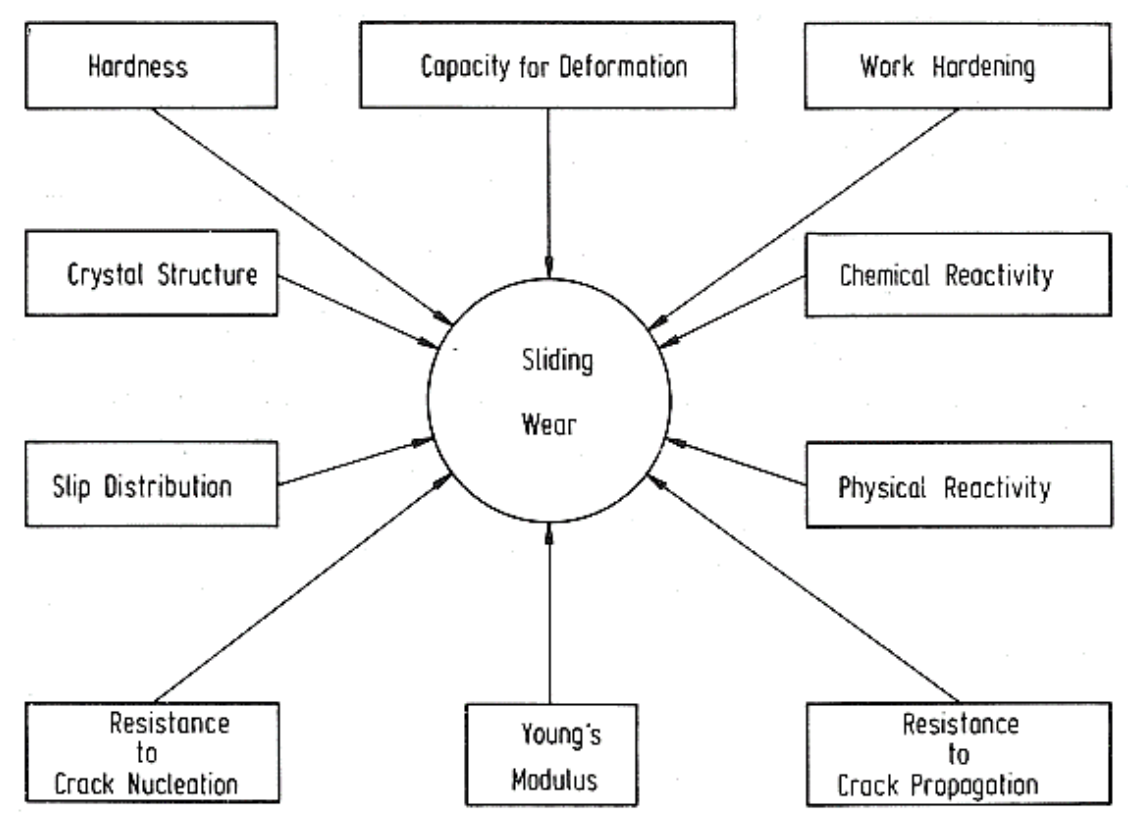

Figure 2-21: Metallurgical properties influencing sliding wear [14].

Phase transformations such as the face-centred cubic (fcc) to hexagonal-close packed (hcp) transformation seen in Stellite alloys (Section 2.5), is of major importance. In terms of metal lattice transformations, as shown in Figure 2-22, it has been found that adhesion increases from hcp to bcc, to fcc [14]. The adhesion coefficient is considered to be the ratio of the force required to break 
the adhesion junctions to the normal loading force at the point where the samples are initially compressed [14].

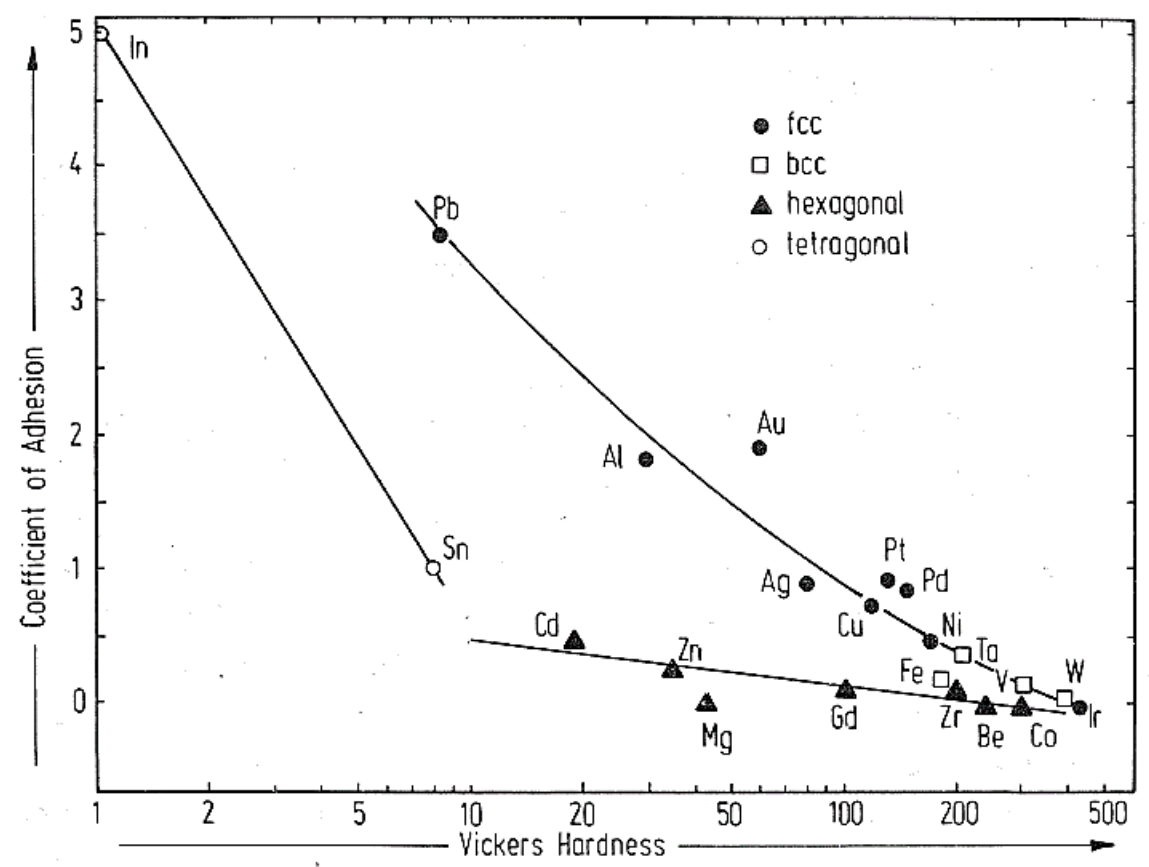

Figure 2-22: Coefficient of adhesion versus hardness of metals [14].

Hardness, although complicated by the different wear mechanisms, is a key factor in the sliding wear process. In severe adhesion, for example, the softer surfaces undergo plastic deformation at lower loads, which increases the area of contact for wear to occur. Material loss occurs in adhesive wear via the rupture of adhesion junctions in the partner of lower cohesive strength [14]. It follows that a harder material increases strength at the adhesion junctions. However, depending on the materials partnered in the sliding wear process, the tendency to adhesion and/or change wear mechanism may mask the effect of hardness [14].

Surface fatigue i.e. crack propagation, is affected by the microstructural elements such as precipitates, soft constituents, and in the case of Stellite alloys, the carbides. Tribochemical 
reactions, such as oxide layers forming tribochemically on the surface, are sensitive to the change in hardness. The underlying material should be able to mechanically support the tribochemically formed layers to prevent the layer breaking down and engaging the wear path. Fracture toughness, i.e., the resistance to crack propagation, and fatigue behaviour of materials under sliding wear conditions, are of great importance. In relatively brittle materials, such as Stellite alloys, crack formation can be easy to achieve with the applied loads in the wear process. It is therefore important to assess and control the growth of cracks. For example, in Stellite alloys this would be through the control of the volume fraction of carbides [2].

Three mechanisms are prevalent in the sliding wear of Stellie alloys, and one or more can act on the wear surfaces during the wear process. The three mechanisms can be summarized as surface oxide control, breakdown of surface oxides, and subsurface fatigue [6].

\subsubsection{Surface Oxidation}

Sliding wear, either through frictional heating or a high-temperature environment, creates a situation where oxide formation and low wear rate are established. Oxide growth rate is increased as the temperature increases in the wear system, and under certain conditions "oxide glazes" can form [6]. Figure 2-23 shows an example of an expected "oxide glaze" on a worn surface. The "oxide glazes" are formed from sheared oxide asperities which have been redistributed over the wear surface. The oxide glazes can act to reduce friction by virtue of the very fine particles generated and the plastic flow associated with the oxide [19]. However, at an arbitrary point in the wear process, the mechanics of the contact area and asperity junctions change, and the oxide starts fracture [19]. This oxide debris can act as an abrasive if caught between the sliding wear surfaces. 


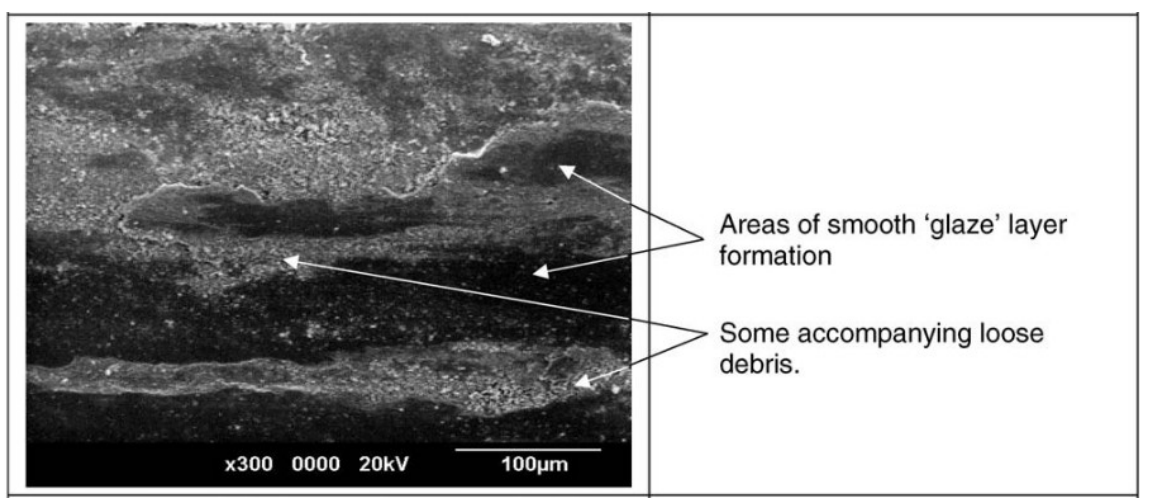

Figure 2-23: SEM Micrograph of Incoloy MA956 worn surface against a Stellite 6 counterfac at $510^{\circ} \mathrm{C}[20]$.

Higher contact stresses acting on the wearing surface can cause the oxide film to breakdown and expose the un-oxidized metal surface. This mechanism has the potential to cause cold welding of wear debris, and with continued movement, the possibility of small pieces breaking free from the wear surface. The term used for this type of damage is galling, and is characterized by gross deformation which results in gouged/torn surfaces and significant metal transfer of wear surface materials [6].

The conditions applicable to subsurface fatigue are similar to that for typical fatigue failures, in that a repeated cyclic stress propagates cracks in the wear material surface to a specific depth. Once fatigue has propagated and surface cracks have developed, the cracked zones then release material into the worn path as the wear process continues. Resisting deformation and fracture, as well as useful oxidation behaviour, are indicative of materials that perform well under sliding conditions [6]. 


\subsubsection{Erosion Wear}

Erosion takes four distinct forms: solid-particle, liquid-droplet, cavitation, and slurry [6]. Solid-particle erosion involves the impingement of small, solid particles against a surface. In this type of wear the particles are typically airborne or entrained in some gaseous environment. Slurry, also known as liquid-solid particle, is similar to solid particle but the carrier fluid is liquid. Cavitation is the repeated nucleation, growth and violent collapse of cavities, or bubbles in a liquid, and liquid-droplet erosion involves impacts on a surface by liquid drops or jets. Although liquid-droplet and cavitation both introduce shock (or stress) waves into the surface, the mechanism is different [6].

In general, the same material properties of Stellite alloys applicable to providing good lowstress abrasion resistance, e.g. hardness, are also found to offer good low-angle solid-particle

erosion resistance [8]. Whereas, in high-angle solid particle erosion situations, fracture toughness is more important [8]. As with all the erosive wear mechanisms, the properties of the erodent are also a factor when evaluating the wear system. Erosion is a complex wear mechanism and work on erosion is not the focus of this thesis.

\subsection{The Archard Wear Equation}

In practice, wear testing is very time consuming, and many iterations have to be performed to ensure that a steady-state wear has been achieved. A simplistic approach to analyzing sliding wear was published by Holm and Archard in the late 1940s and early 1950s, and the model contains a number of assumptions surrounding the following [21]:

i) Size and distribution of the total area of contact

ii) Duration of any given contact 
iii) Shape of the worn particles

iv) Probability factor

Using the work of Holm, and considering numerous assumptions, a simple mathematical approach to modeling the severity of wear was derived by Archard [21][22]. The starting point of the model is the assumption that contact occurs when asperities on opposing surfaces touch, and that the sum of the individual asperity contact areas is the true area [23]. The true area is closely proportional to the normal load, and under most conditions, it can be assumed for metals that most of the asperities will succumb to plastic deformation [23]. As shown in Figure 2-24(a), this is the maximum contact area of radius, $a$. Figure 2-24(b) shows the contact area after sliding through distance $y$, and Figure 2-24(c) shows zero contact area after sliding through a distance, $2 a$.

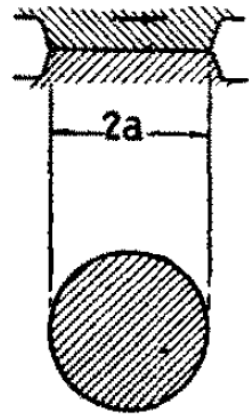

(a)

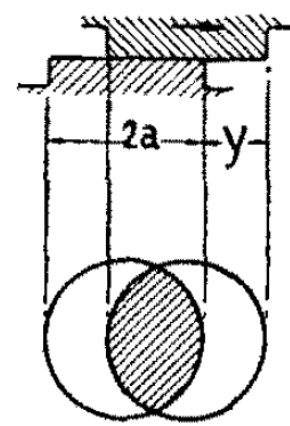

(b)

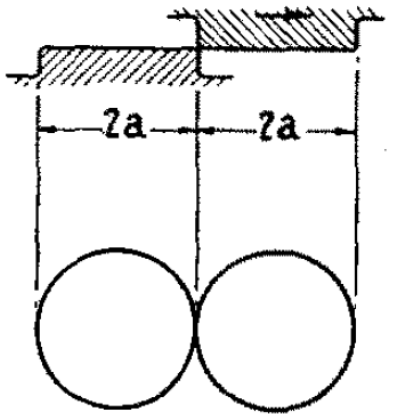

(c)

Figure 2-24: Idealized representation of single contact in sliding surfaces, side view (above) and top view (bottom) [21].

Assumption is made that the asperity contact is circular. In Figure 2-24(a), at the maximum contact area, and the normal load per unit sliding distance, $P_{u s d}$, acting on the asperity is given by:

$$
P_{u s d}=p_{m} \pi a^{2}
$$


where, $p_{m}$, is the yield pressure (flow stress) for the plastically deforming asperity. This value is close to the hardness, $H$, of the material so that it can be considered the same [23]. This near equivalency comes from the principles of indentation hardness testing. When deformation occurs during hardness testing and this deformation is fully plastic in nature, the mean indentation pressure is used as a measure of indentation hardness of the material [23].

As sliding continues, this single asperity to be free of the original load which has, at this point, been transferred to other forming asperity junctions, as illustrated in Figure 2-24(c). The process of forming and destruction of asperities progresses as the wear process continues. Wear implies the detachment of material from the surface asperities, and the volume of material loss will depend on the size of the asperity junction where the material loss has occurred. The assumption is that the volume of material removed is proportional to the cube of the contact dimension, $a$ [23], and the shape of the wear particle should be independent of its size [21]. In his work, Archard assumed that the wear particles are hemispherical in shape having the same radius, $a$, with the contact areas. Therefore, the volume of a unit particle, $V_{p}$, is:

$$
V_{p}=\frac{2 \pi a^{3}}{3}
$$

Not all asperity junctions generate wear particles under loading, and Archard assumed that a proportion, $\mathrm{k}$, does so [21]. Over a distance of $2 a$ (Figure 2-24a), this provides a value for the volume of material, $V$, worn away per unit sliding distance:

$$
V=\frac{\mathrm{k} V_{p}}{2 a}=\frac{\mathrm{\kappa} \pi a^{2}}{3}
$$


The sum of the individual contributions over the whole real area of contact will give the worn volume per sliding distance, $w$ :

$$
w=\sum V=\frac{\mathrm{K}}{3} \sum \pi a^{2}
$$

Using Eq. (2.1), the total normal load, $P$, becomes:

$$
P=\sum P_{u s d}=p_{m} \sum \pi a^{2}
$$

By rearranging Eq. (2.5) for $\sum \pi a^{2}$, and then substituting into Eq (2.4), this will relate the worn volume in unit sliding distance to give:

$$
w=\frac{\mathrm{k} P}{3 p_{m}}
$$

Combining the factor $\frac{1}{3}$ into the constant of proportionality $K$ by using, $=\frac{\mathrm{K}}{3}$, Eq. (2.6) becomes:

$$
w=\frac{K P}{p_{m}}
$$

where $K$ is referred to as the wear coefficient (or, coefficient of wear) and assumes that, $P$ is the applied load, and $p_{\mathrm{m}}$, is flow pressure of the softer material. As previously discussed, the flow pressure, $p_{m}$, can also be considered as the hardness of the softer material, $H$. To calculate the 
worn volume over larger distances instead of a unit sliding distance, the total sliding distance, $s$, should be multiplied, i.e., $W=w \cdot s$, where $W$ is the worn volume. Then, Eq. (2.7) becomes:

$$
W=\frac{K s P}{H}
$$

This equation depicts a fundamental principle in sliding wear mechanisms that hardness is directly related to wear loss and is widely known as the Archard Equation. This equation suggests that if the material hardness increases, the worn volume decreases. In engineering applications, the quantity $\frac{K}{H}$ is represented as $k$ which denotes the dimensional wear coefficient [23]. This value represents the volume of material removed by wear per unit distance slid, per unit normal load on the contact [23]. The dimensional wear coefficient is useful for comparing wear rates in different classes of materials. Many systems have shown that wear is proportional to the sliding distance as shown in Figure 2-25. The materials are [22]: (1) Teflon on hardened tool steel under 150 g. (2) 60/40 brass on hardened tool steel under 120 g. (3) Ferritic stainless steel on sintered tungsten carbide under 1200 g. (4) Beryllium copper on hardened tool steel under 1000 g. (5) Stellite Grade 1 on hardened tool steel under 750 g. (6) Sintered tungsten carbide on mild steel under $1000 \mathrm{~g}$. However, transient behavior can occur until the system reaches a steady-state, the process depends on the nature of the running-in process as previously discussed. 


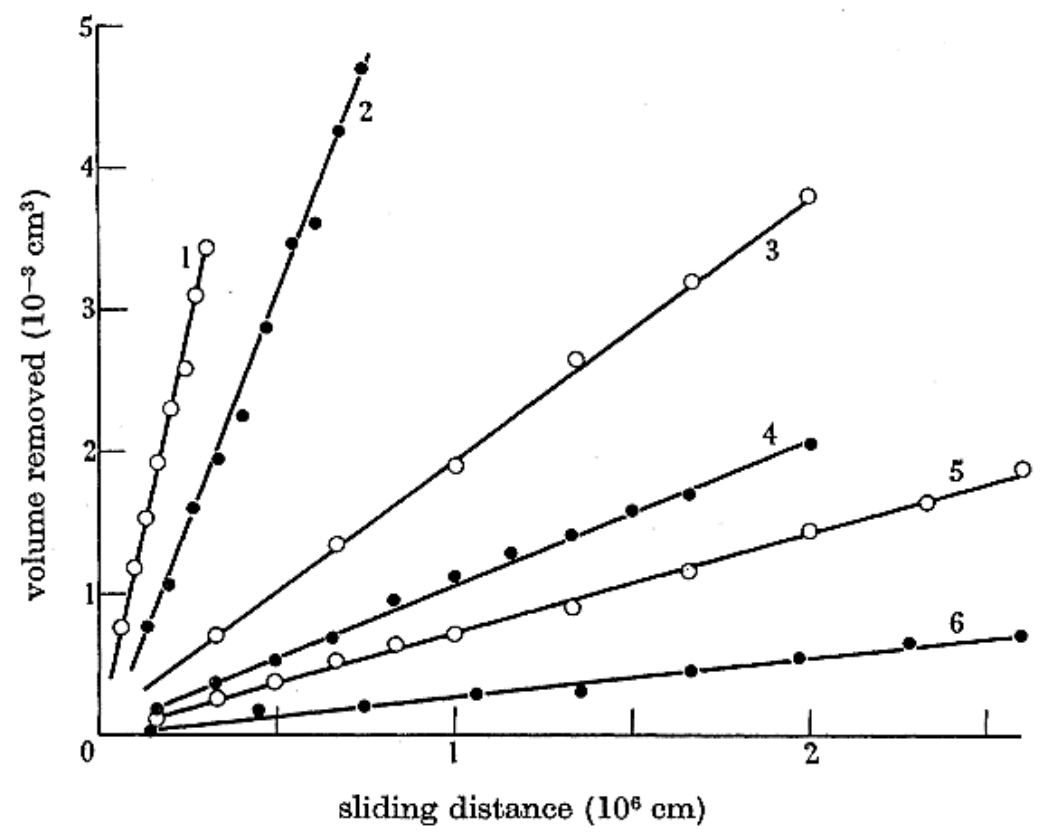

Figure 2-25: Wear vs. sliding distance for pins rubbing on rings of different materials (speed $180 \mathrm{~cm} / \mathrm{s})[22]$.

The constant $\mathrm{K}$ is understood to use the probability of a wear particle being produced from an asperity junction [21], thus while it would be ideal to have one wear equation, it is important to note that other interpretations for $\mathrm{K}$ can exist. For example, $\mathrm{k}$ could relate to the number of cycles before a fragment of material is removed [23].

\subsubsection{Sliding Wear Modeling}

Many other sliding wear models have been developed since Archard and Holm published their work. Sarkar [24] presented an extension to the Archard equation which incorporates the friction coefficient, $\mu$, and can be written with respect to Eq. (2.8) as:

$$
\frac{W}{S}=k \frac{P}{H} \sqrt{1+3 \mu^{2}}
$$


Another wear model proposed by Hegadekatte et al. [25] utilized a Global Incremental Wear Model (GIWM) to find the wear coefficient from pin-on-disc data, with a view to also predicting the wear depth within a limited range of parameter variations. They performed experiments using self-mated silicon nitride, $\mathrm{Si}_{3} \mathrm{~N}_{4}$, and tungsten carbide-cobalt, WC-Co materials on a pin-on-disc apparatus in an unlubricated environment at three different loads. Using the GIWM, the results of the actual data were compared with the computational data and are presented in Figure 2-26, where $h^{w}$ is the wear depth. The model appeared to show good correlation for the $200 \mathrm{mN}$ and $400 \mathrm{mN}$ loads, but showed an increased $h^{w}$ for $800 \mathrm{mN}$ as the distance, $s$, increased $[25]$.

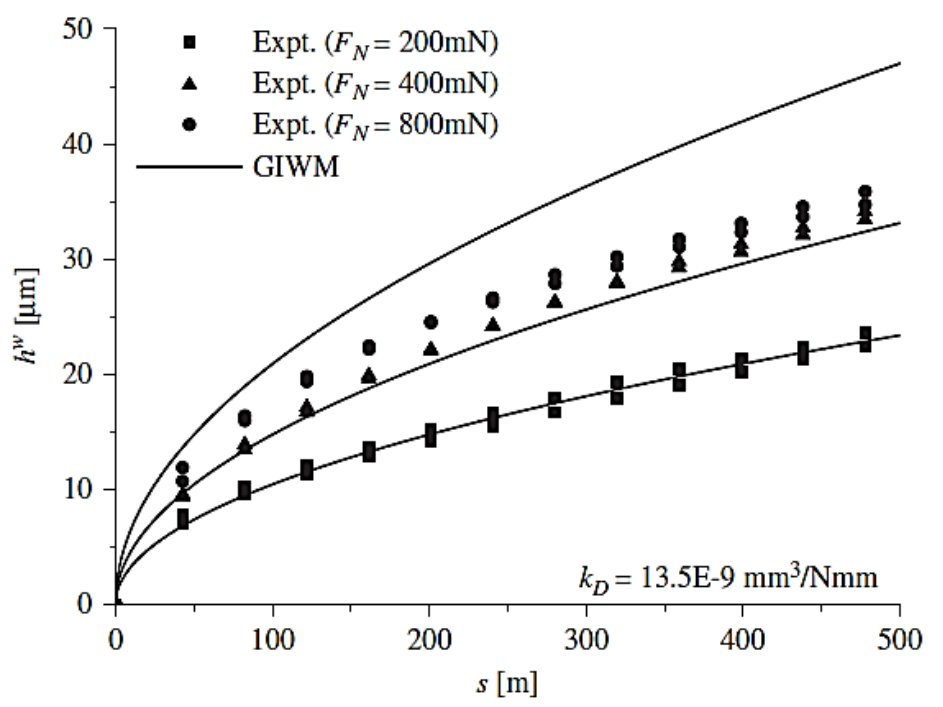

Figure 2-26: Predictions from the GIWM in comparison with the experimental results [25].

Evaluation by Hegadekatte et al. in Figure 2-26 indicates that the piezo electric sensor used in the experiment may not have been adequate to record results correctly at the higher $800 \mathrm{mN}$ 
load [25]. This result highlights one of many difficulties in wear testing to achieve consistent and accurate results in order to model wear loss.

McMarron et al. investigated the wear of cobalt-based alloys in nuclear reactor conditions, and formulated a wear prediction model using both Hertzian contact mechanics and the Archard wear equation [26]. The results showed the wear prediction model to be in fairly good agreement with respect to the total wear depth for the ball scar and disc track [26] in experiment. However, the model was limited in terms of predicting the width of the wear scar, due to the wear track not being uniform on the actual samples [26].

Modeling of sliding wear has been extended into modeling of both the pin and disc. Zhang et al. studied the running-in wear of a steel/ $\mathrm{SiC}_{\mathrm{p}}-\mathrm{Al}$ composite system [27]. Two variations of a 2014 aluminum matrix composite pin, reinforced with silicon carbide particles with average diameters of 13 and $37 \mu \mathrm{m}$, were run against a carbon steel with a hardness of HV450. They considered the abrasive process and the probability of fractured particles to generate a model to predict the volumetric wear loss of the steel disc. The equation is presented as [27]:

$$
V_{s}=\frac{\beta P d f_{v}}{k_{1} H_{s}}\left[1-\exp \left(-k_{1} \frac{L}{d f_{v}}\right)\right]
$$

where $V_{s}$ is volumetric wear of steel, $\beta$ is a wear coefficient, $P$ is the load, $d$ is the average particle diameter, $f_{v}$ is the particle volume fraction, $k_{1}$ is a proportional constant, $H_{s}$ is hardness of the steel, and $L$ is the sliding distance. The results of using this running-in (transient) wear model are illustrated in Figure 2-27, where the theoretical model provides reasonable predications relative to the obtained data [27]. 


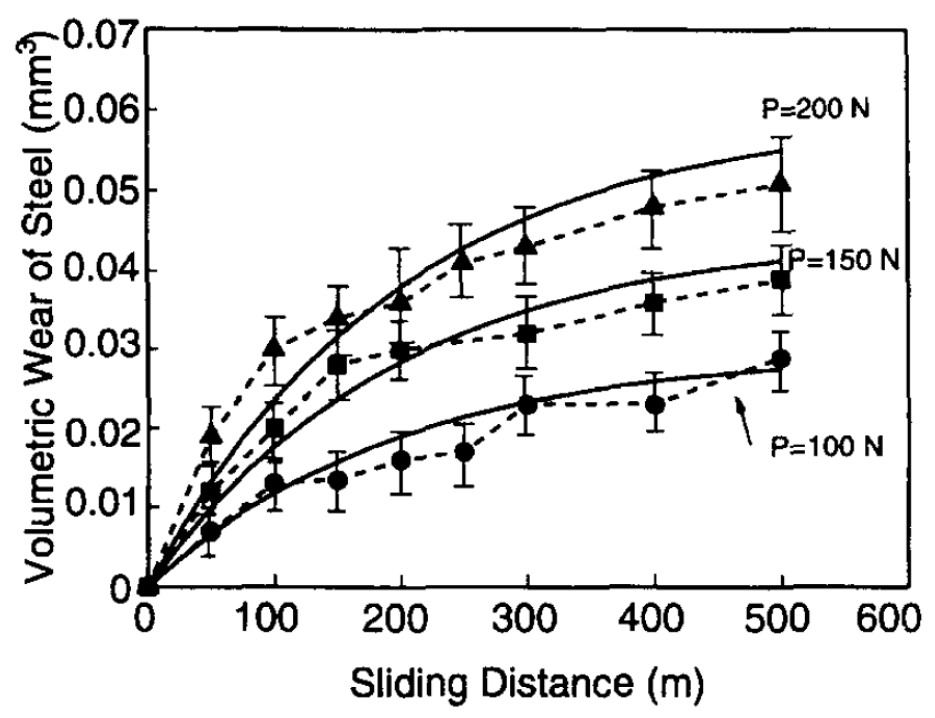

Figure 2-27: Comparison of theoretical predictions (solid line) with experimental results (shaped points) of steel disc wear at different loads [27].

Certain assumptions were made to generate this model, which among others, included that abrasion is the dominant wear mechanism for the steel disc, and consequences generated by other mechanisms can be neglected [27]. Zhang et al. also presented a wear volume model for the composite pin at any given distance [27]:

$$
V_{c}=\frac{g_{1}}{g_{3}} \frac{P}{H_{c}} \frac{d\left(1-f_{v}\right)}{f_{v}}\left[1-\exp \left(-g_{3} \frac{L f_{v}}{d\left(1-f_{v)}\right.}\right)\right]
$$

where, in addition to the previous nomenclature, $V_{c}$ is the wear volume of the composite, $g_{1}$ and $g_{3}$ are constants, and $H_{c}$ is the composite hardness. The results using this running-in (transient) wear model are shown in Figure 2-28, where again, the theoretical model provides reasonable predications relative to the obtained experimental data [27]. 


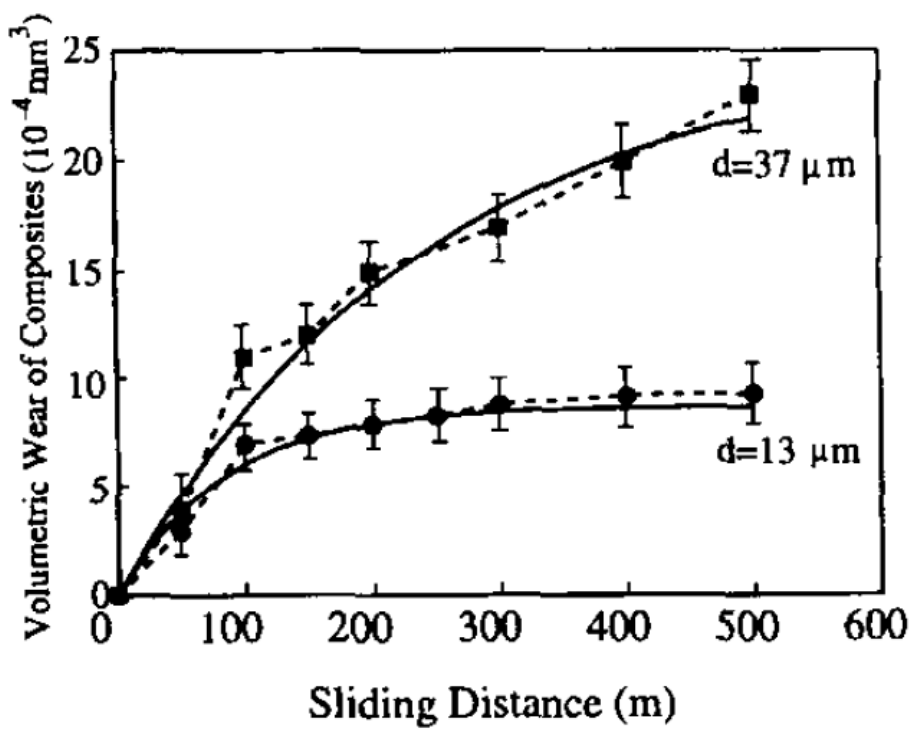

Figure 2-28: Comparison of theoretical predictions (solid line) with experimental results (shaped points) of composite pin wear at a normal load of $150 \mathrm{~N}$ [27].

\subsection{Phase Transformation in Stellite Alloys}

Upon slow cooling from high temperatures, cobalt undergoes what is considered a sluggish allotropic phase transformation from fcc ( $\alpha$ phase), to a hep ( $\varepsilon$ phase) phase at around $422^{\circ} \mathrm{C}$ [8][28]. Hence, in practice, Stellite alloys form a metastable fcc crystal structure. This fcc to hep transformation is classified as martensitic, i.e. occurring by shear, and is essentially athermal in nature [28]. The wear resistance of cobalt-based alloys can be attributed to both the formation of carbides, and the deformation response of the cobalt-rich crystal lattice [8]. The deformation response is quite significant in that Stellite alloys possess what is known as a low stacking fault energy.

Stacking faults are discontinuities in the stacking order of the close-packed plane i.e. the $\{111\}$ plane, with certain surface energy being associated with this stacking fault [29]. Taking into consideration the metastable fcc phase found in Stellite alloys, by introducing energy (stress) into the crystal structure via the forces during wear deformation, the metastable fcc crystal structure 
will be encouraged to transform to hcp [8]. This transformation is important because it not only occurs at room temperature in Stellite alloys exhibiting a stacking fault energy less than $15 \mathrm{~mJ} / \mathrm{m}^{2}$, but also means that with less operative slip systems, the hcp phase can resist deformation to a greater degree than the metastable fcc phase [28]. This results in Stellite alloys being able to fracture rather than plastically deform in metal-to-metal wear applications, leading to both reduced galling and low friction characteristics.

This characteristic is of significant importance when Stellite alloys are subjected to external forces, such as those experienced in wear systems. The alloys can effectively defend themselves from the opposing forces by transforming the microstructure under the attack.

\subsection{Sliding Wear of Stellite Alloys}

Sliding wear introduces many different types of interactions in the wear zone. These include a combination of high contact stresses, a wear groove that can entrap debris thus reducing the wear rate (applicable to steady-state wear), and the formation and removal of a tribofilm [8]. In general, hardness is not directly related to sliding wear resistance [8], which makes understanding the wear process of upmost importance in recommending an alloy for a particular application.

The combined effort of the fcc-to-hcp matrix transformation and the carbides in Stellite alloys is important for wear deformation. The matrix must resist deformation and subsequent removal in order to support the carbides, and the carbides themselves must resist the onslaught of the wear process by having suitable properties for the application. The carbide size and morphology are the key factors to differentiate carbide-containing cobalt-based alloys [8]. 
Extensive work on the sliding wear of Stellite alloys has been well documented. One example is the generation of a sliding wear map of laser clad Stellite $6(1.2 \mathrm{wt} . \% \mathrm{C})$ using a pinon-disc type machine and a hard metal (90 wt.\% WC - $10 \mathrm{wt} . \% \mathrm{Co})$ mating disc [30][31]. The wear map in Figure 2-29 shows the sliding conditions (basal plane) and the wear rate along the vertical axis. As previously discussed, the carbide formation, and hence hardness in Stellite alloys, is highly dependent on the manufacturing process. The Stellite 6 microstructures which were generated from different manufacturing processes and used in the work of Frenk and Wagnière highlighted that, in this particular case, no direct relationship was found between the initial hardness of the samples and the resulting wear resistance [31].

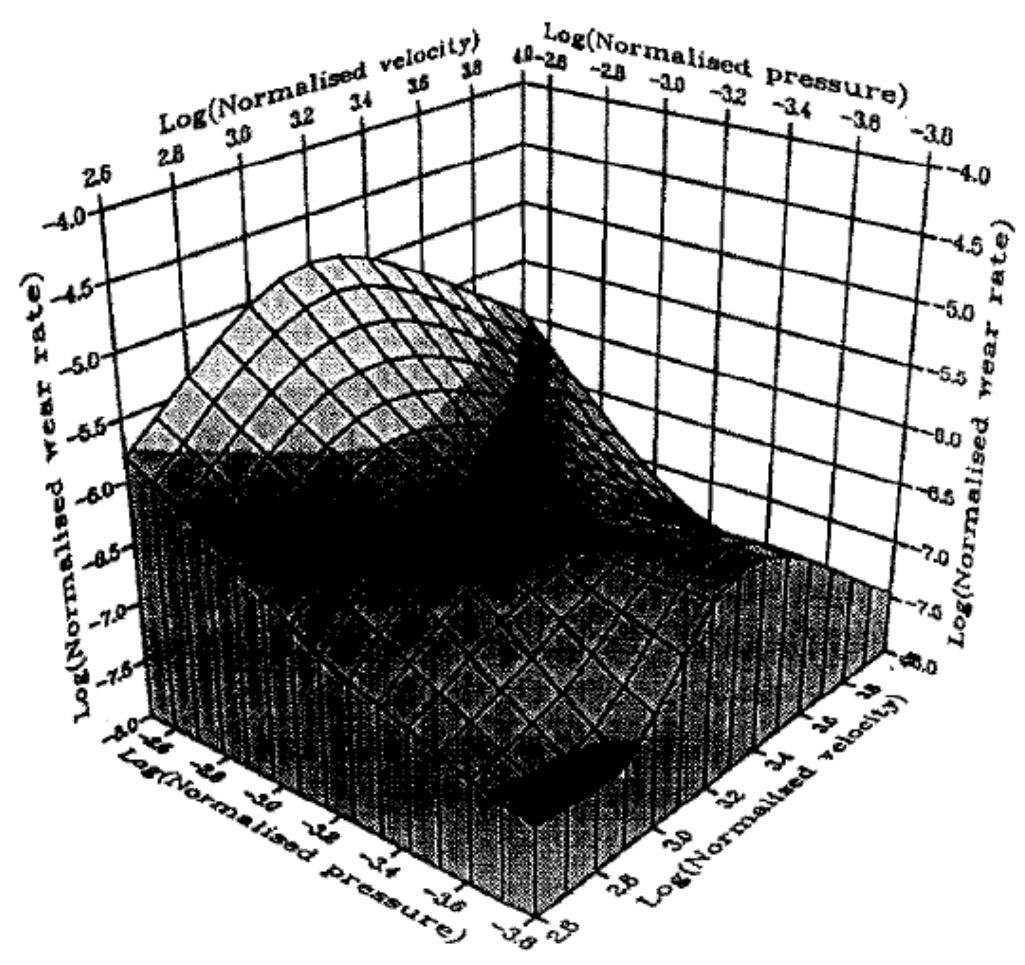

Figure 2-29: Dry sliding wear map for Stellite 6 against a WC-Co 90/10 disc [31].

A low friction coefficient is one attribute of Stellite alloys that contributes to its excellent resistance to sliding wear. Test data reported by Yao et al. demonstrated that in an ASTM G77 
block on ring test, the Mo-containing Stellite alloys (Stellite 700 series) had a slightly higher coefficient of friction (Table 2-2), and yet the adhesive wear was comparable with, and in some cases better than the W-containing Stellite alloys (Figure 2-30) [32].

Table 2-2: Coefficients of friction obtained during adhesive test [32]

\begin{tabular}{|c|c|c|c|c|c|c|}
\hline Alloy & Stellite 12 & Stellite 712 & Stellite 1 & Stellite 701 & Stellite 190 & Stellite 790 \\
\hline Run 1 & 0.363 & 0.402 & 0.432 & 0.465 & 0.421 & 0.483 \\
\hline Run 2 & 0.445 & 0.465 & 0.463 & 0.462 & 0.448 & 0.472 \\
\hline Average & 0.404 & 0.434 & 0.448 & 0.464 & 0.435 & 0.478 \\
\hline
\end{tabular}

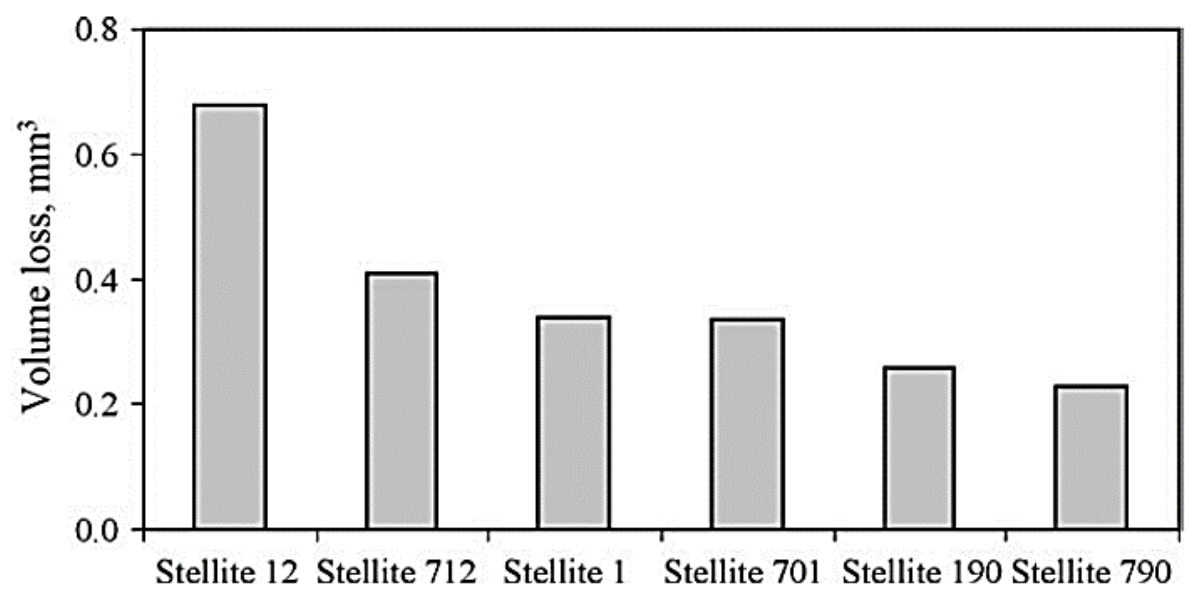

Figure 2-30: Adhesive-wear resistance of Stellite alloys, ASTM G77, S10 type ring and test duration 2003 cycles, under $150 \mathrm{lbs}$ load [32].

In a sliding wear test to compare three Mo-containing Stellite alloys having different volume fractions of carbides, Liu et al. showed that an increased volume fraction of carbides significantly improved the wear resistance [3]. Stellite 706 has the lowest volume fraction of carbides in this group of alloys, and Stellite 720 the highest, with Stellite 712 falling between these two (Figure 2-31). 


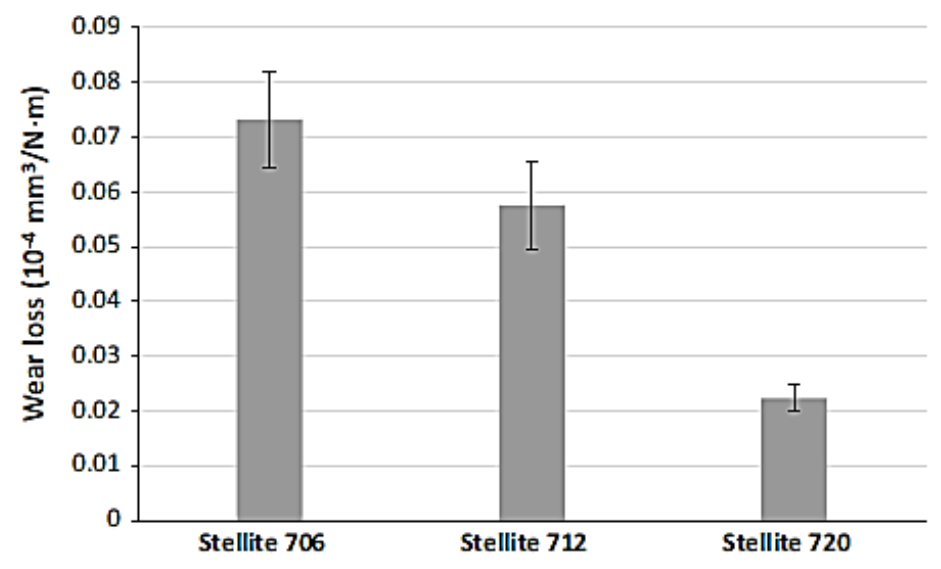

Figure 2-31: Sliding wear resistance of 700 series Stellite alloys, Pin-on-Disc WC Ball, 10 N, $350 \mathrm{rpm}, 2.5 \mathrm{hrs}[3]$.

This work also showed a consistent relationship between hardness and wear resistance, i.e. wear resistance increases with hardness. However, when compared with their complementary alloys containing W instead of Mo, Liu et al. demonstrated that Stellite 720 and Stellite 706 do not perform as well under the same conditions even though the hardness of the respective alloys is similar [3]. Figure 2-32 shows the hardness values for comparable W (Stellite 6 and Stellite 3) and Mo (Stellite 706 and Stellite 720) alloys.

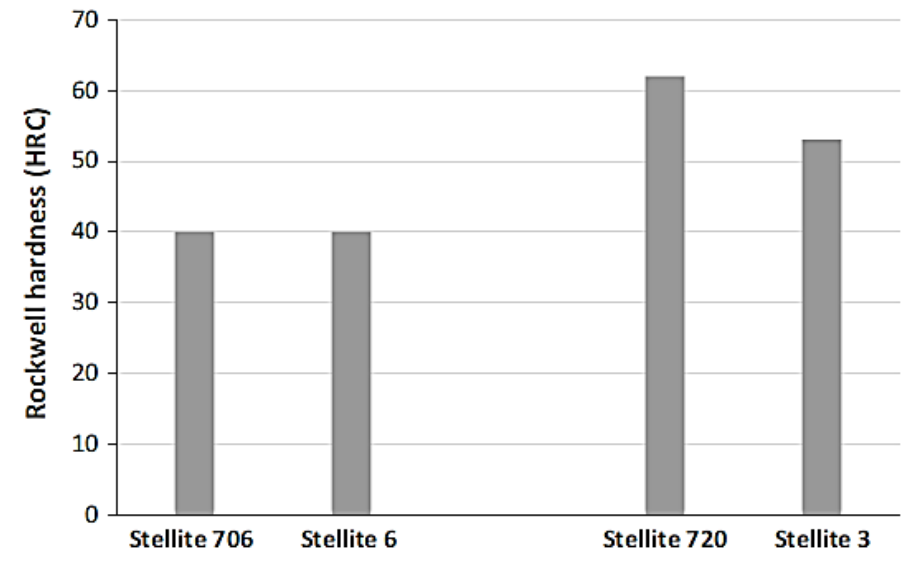

Figure 2-32: Comparison of hardness between CoCrMo and CoCrW Stellite alloys [3]. 
In addition, even though Stellite 720 is the hardest alloy in this particular group of Stellite alloys, it performed worse than Stellite 3 in this test, as shown in Figure 2-33 [3]. Table 2-3 shows that the Mo-containing Stellite alloys in this group are similar in carbide volume fractions as their W-containing counterparts.

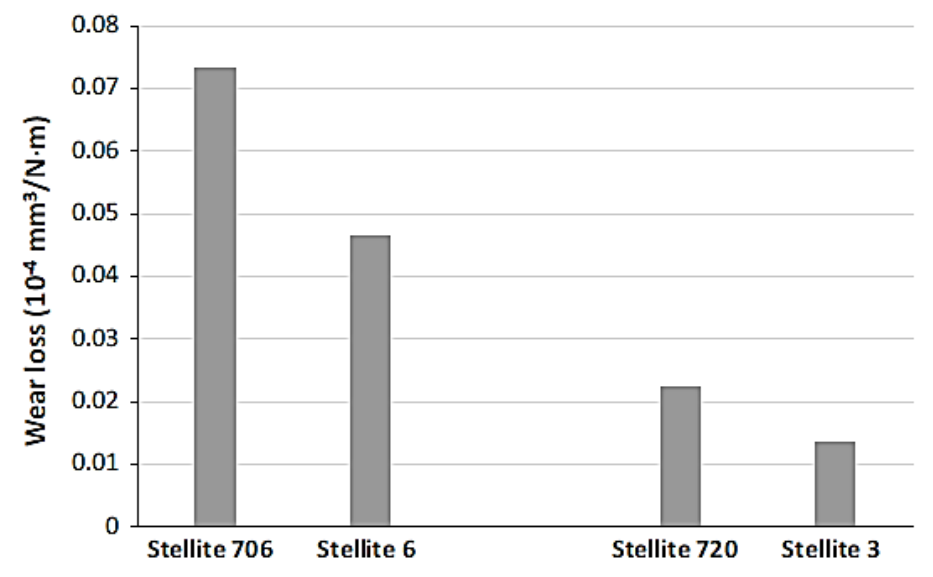

Figure 2-33: Comparison of wear resistance between CoCrMo and CoCrW Stellite alloys[3].

Table 2-3: Summary of volume fractions (pct) of each phase in Stellite Alloys [3]

\begin{tabular}{|c|c|c|c|}
\hline Alloy & $\begin{array}{c}\text { Mo-rich carbides or } \\
\text { W-rich carbides }\end{array}$ & $\begin{array}{c}\text { Cr-rich } \\
\text { carbides }\end{array}$ & $\begin{array}{c}\text { Co solid } \\
\text { solution }\end{array}$ \\
\hline Stellite 706 & 2.64 & 13.91 & 83.45 \\
\hline Stellite 712 & 5.38 & 24.26 & 70.36 \\
\hline Stellite 720 & 19.6 & 25.09 & 55.31 \\
\hline Stellite 6 & 1.32 & 15.57 & 83.11 \\
\hline Stellite 3 & 5.65 & 27.83 & 66.52 \\
\hline
\end{tabular}

Stellite 6 , containing $\mathrm{W}$, has a volume fraction of $15.57 \% \mathrm{Cr}$-rich carbides, and the counterpart Mo-containing Sellite 706 has 13.91\% Cr-rich carbides (Table 2-3). This leaves the solid solution matrix to be the differentiating factor between the alloys when considering 
similarities in carbide volume fractions. This study highlighted the importance of understanding the different phases and the type of wear experienced in the tribological system.

\subsection{Summary}

Stellite alloys form a group of wear, heat, and corrosion resistant alloys that are specifically formulated to address all three of these material degradation concerns. The primary element in Stellite alloys is cobalt, with other alloying elements such as chromium, tungsten, carbon and molybdenum.

The properties of Stellite alloys can be very diverse depending on the alloying elements and processing history $[8,9]$. Based on the literature, Stellite alloys can be grouped based on either their microstructure (hypoeutectic and hypereutectic) [4], or, their intended use by grading the carbon content as high, medium or low carbon. The amount of carbon in Stellite alloys governs the type and amount of carbides that form $[4,6,7]$, hence the reasoning behind the high, medium and low grading. It was suggested that the higher carbon Stellite alloys having a higher volume fraction of carbides lend themselves to more wear type applications, while the lower carbon alloys having less carbides are used more for corrosion type applications $[1,2,4,9,10]$. In addition, the different type and size of carbides, and how they form will impart particular properties on the alloy, for example blocky carbides provide better protection against abrasive wear [3].

Abrasive, sliding and erosive wear induce damage via different mechanisms $[1,3,12,13]$, and all can happen to Stellite alloys. It must be recognized that the lines drawn between each wear process are dynamic, as what begins as one mechanism change into another $[12,13,17]$. The important factor for wear of Stellite alloys is the oxide glaze/layer that forms on the surface, and how this layer behaves with the prevailing wear mechanism(s) [4]. 
For wear modeling, the Archard equation was especially discussed with regards to sliding wear. This equation $[20,21,22]$, is considered to be a starting point for analyzing sliding wear. In the ensuing years since his work, numerous wear equations with many different parameters have been developed $[13,20,21,22,24,25,26]$. There is no simple universal model available that can predict wear on the basis of mechanical properties and contact information only.

It is therefore important to understand the mechanisms behind the sliding wear process, and how the process can evolve from one mechanism to another. In addition, semi-analytical models of the sliding wear process, whilst cheaper to produce, have the limitation of overlooking certain aspects of the wear process with certain assumptions being made. That being said, although data exist for Stellite alloys under various wear scenarios $[4,10,11,25,29,30,31]$, the present research is aimed at understanding a newer generation of Stellite alloys that employ molybdenum instead of tungsten under dry sliding wear conditions. Molybdenum can enhance wear resistance and assist in corrosion resistance in reducing environments $[9,11]$. The basis for the study is to combine the Archard's equation with a transient type wear model to understand the wear of three molybdenum-containing Stellite alloys. 


\section{Microstructural Characterisation}

\subsection{Specimen Preparation}

\subsubsection{Alloy Selection}

As mentioned previously, Stellite alloys can be categorized either metallurgically (hypoeutectic or hypereutectic) or by the carbon content (high, medium or low). The carbon dictates the volume fraction of carbides, as well as the type and how carbides form in the microstructure. Therefore, in Stellite alloys, with carbon ranging from $\sim 0.1 \mathrm{wt} . \%$ up to $\sim 3 \mathrm{wt} \%$ a large variation in carbide content is plausible. The volume fraction of carbides governs the material properties, for example, hardness, which is important for wear applications. Tungsten is primarily utilized in Stellite alloys to provide solid solution strength and carbide formation but can be replaced by Mo which also partitions to the carbides. If the same weight percentage of $\mathrm{W}$ is exchanged for Mo, there will be an excess of Mo due to the atomic mass difference. This in turn will affect the formation of carbides in the molybdenum Stellite alloys. It is both the range of $\mathrm{C}$ and exchange of Mo that governs the selection of materials for this research.

\subsubsection{Chemical Composition}

Three Mo-containing Stellite alloys were selected for this study with varying carbon and molybdenum contents. They are Stellite 21, Stellite 720 and Stellite 728, and are all cobalt-based. The nominal chemical compositions (wt.\%) of these alloys are given in Table 3-1. 
Table 3-1: Chemical compositions of selected Stellite Alloys

\begin{tabular}{|c|c|c|c|c|c|c|}
\hline \multirow{2}{*}{ Alloy } & \multicolumn{6}{|c|}{ Element (wt.\%) } \\
\cline { 2 - 8 } & Co & C & Cr & Mo & Ni & Other \\
\hline Stellite 21 & Bal. & 0.25 & 27 & 5.5 & 2.5 & \\
\hline Stellite 720 & Bal. & 2.5 & 33 & 18 & 1.5 & \\
\hline Stellite 728 & Bal. & 0.3 & 24 & 12 & 4 & $2 \mathrm{Nb}$ \\
\hline
\end{tabular}

The carbon content varies from $0.25 \sim 2.5$ wt. $\%$, chromium content between $24 \sim 33$ wt.\%, molybdenum content between $5.5 \sim 18$ wt.\%, and one alloy, Stellite 728 , has the addition of 2 wt.\% niobium. These alloys also contain small amounts of nickel which is an fcc stabilizer and therefore supresses the fcc to hcp transformation allowing more work hardening effects. Nickel also contributes to the solid solution strengthening of Stellite alloy. Stellite 21 is considered the benchmark of Co-Cr-Mo based Stellite alloys. This alloy is one of the most resistant to corrosion by virtue of the low carbon content allowing most of the chromium to remain in the solid solution. Stellite 720 having the highest carbon, has large amounts of carbides and less solid solution which are more applicable to wear-resistant properties. Stellite 728 is the newest alloy of all three used in this research. This alloy is a step up from Stellite 21 in that the higher molybdenum content is added for corrosion, and niobium is added to tie up the carbon to form niobium carbides for improved wear resistance. 


\subsection{Specimen Surface Preparation}

In order to study the wear properties of the molybdenum Stellite alloys, it is necessary to understand the microstructure first. All three alloys were investment cast at Kennametal Stellite Inc. The sample preparation for microstructural analysis was as follows:

1) The samples were sectioned to achieve an approximate size of $20 \times 15 \times 5 \mathrm{~mm}$ using a semi-automated cutting machine with a diamond-edged blade. Coolant was used along with a slow cutting feed rate $(0.008 \% / \mathrm{sec})$ to minimize distortion and to not overheat the samples that may alter the microstructure. The specimens were then mounted in hot mounting resin with carbon filler. The CitoPress-10 manufactured by Struers (Figure 3-1) was used to mount the samples.

2) The samples were then ground and polished using a Struers Tegramin-30 automatic grinding/polishing machine (Figure 3-2). The polishing procedure started with an initial grinding step using 240 grit silicon carbide papers, followed by a polishing step using a MD-Plan (woven polyester) polishing cloth with DiaPro Plan $9 \mu \mathrm{m}$ diamond suspension, then a final step was taken to achieve a mirror-like surface using a MD-Dur (satin woven natural silk) polishing cloth with DiaPro Dur $3 \mu \mathrm{m}$ diamond suspension. Specimens were cleaned with water, cotton wool and isopropanol between each step.

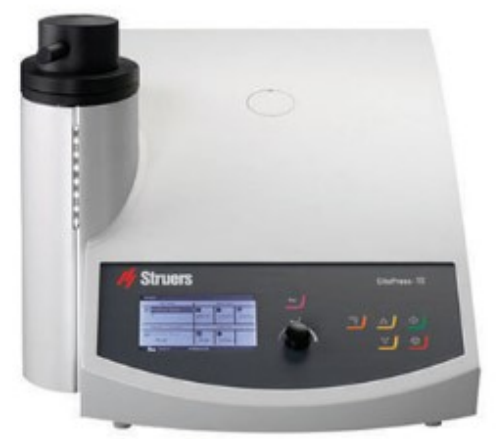

Figure 3-1: Struers Cito-Press for hot resin mounting (Struers Inc). 


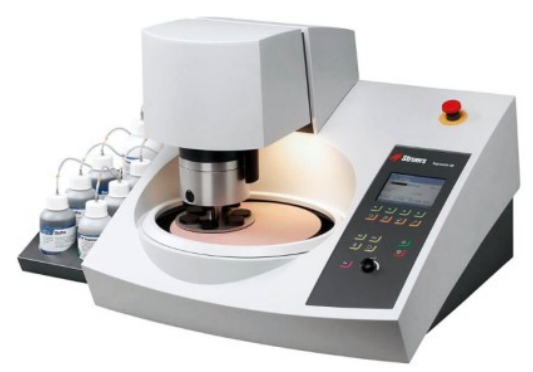

Figure 3-2: Struers Tegramin for Griding and Polishing Mounted Samples (Struers Inc).

\subsection{Microstructure Examination}

The specimens were evaluated using differential interference contrast (DIC) on an Olympus optical microscope. After an optical low magnification view using DIC, the microstructure was further evaluated using a Tescan Scanning Electron Microscope (SEM) with backscatter electron (BSE) imaging to resolve the carbides. To complement the BSE analysis, the SEM is fitted with an energy dispersive $\mathrm{x}$-ray spectroscopy (EDX) unit to qualitatively analyze the different phases by showing the elements present at each selected site for analysis. In addition, $\mathrm{x}$ ray diffraction (XRD) was used to complement the SEM/EDX analysis to accurately identify phases. The samples for SEM/EDX were mounted in conductive resin and polished to a 1 micron finish. The samples remained in the un-etched condition to keep the carbides intact for SEM/EDX analysis. The XRD samples were roughly ground using up to 400 grit sand paper.

The microstructural analysis of the three Stellite alloys shows a great deal of complexity and various phases to be present. The term 'complex carbide(s)' is considered in the current research work as having multiple elements present as part of the carbide. For example, the carbide $\mathrm{M}_{23} \mathrm{C}_{6}$ could have a few different elements forming in the $\mathrm{M}$ part of the carbide. A variety of intermetallics have also been found. 


\subsubsection{Optical DIC Images}

The microstructures were viewed using an Olympus optical microscope with DIC to enhance the carbide structure. This technique gives a pseudo-3D effect so that the carbides appear to pop out of the polished sample without the need for etching to reveal the phases. The effect uses the relief on the sample surface from polishing, i.e. the different phases sit at slightly different heights after polishing. The micrographs are presented in Figure 3-3, Figure 3-4, and Figure 3-5 for Stellite 21, Stellite 720, and Stellite 728 respectively. The images show the expected microstructures of the chosen Stellite alloys, having varying degrees of complex wear-resistant carbides.

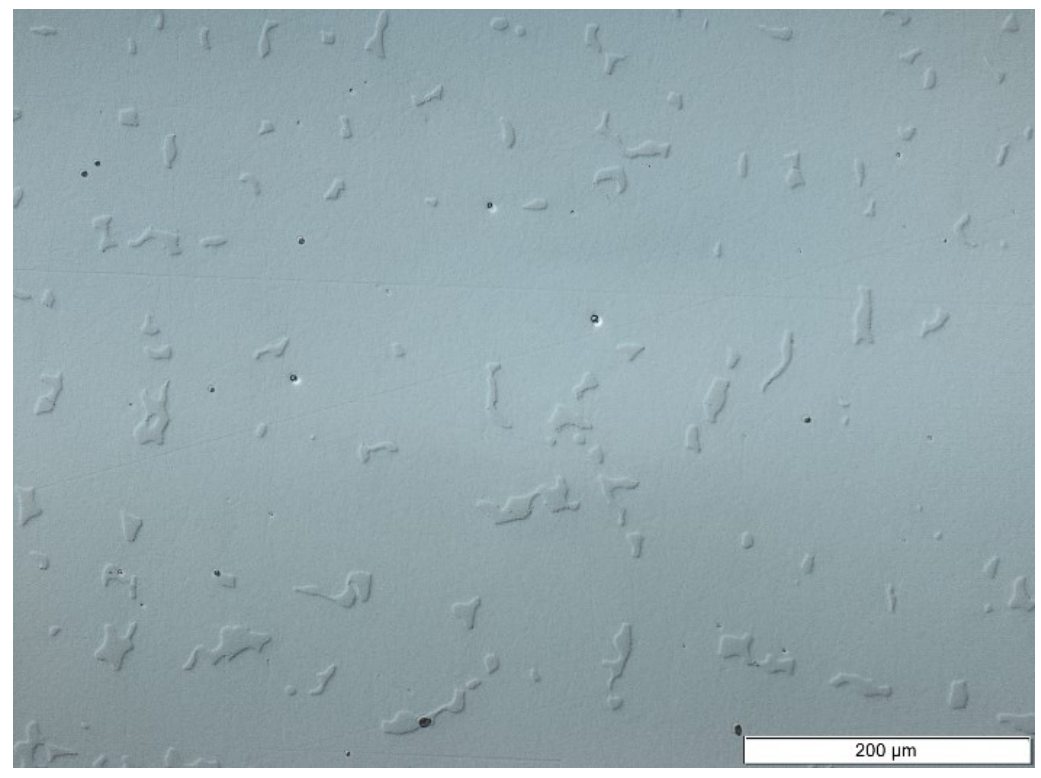

Figure 3-3: Optical microstructure of as-cast Stellite 21. 


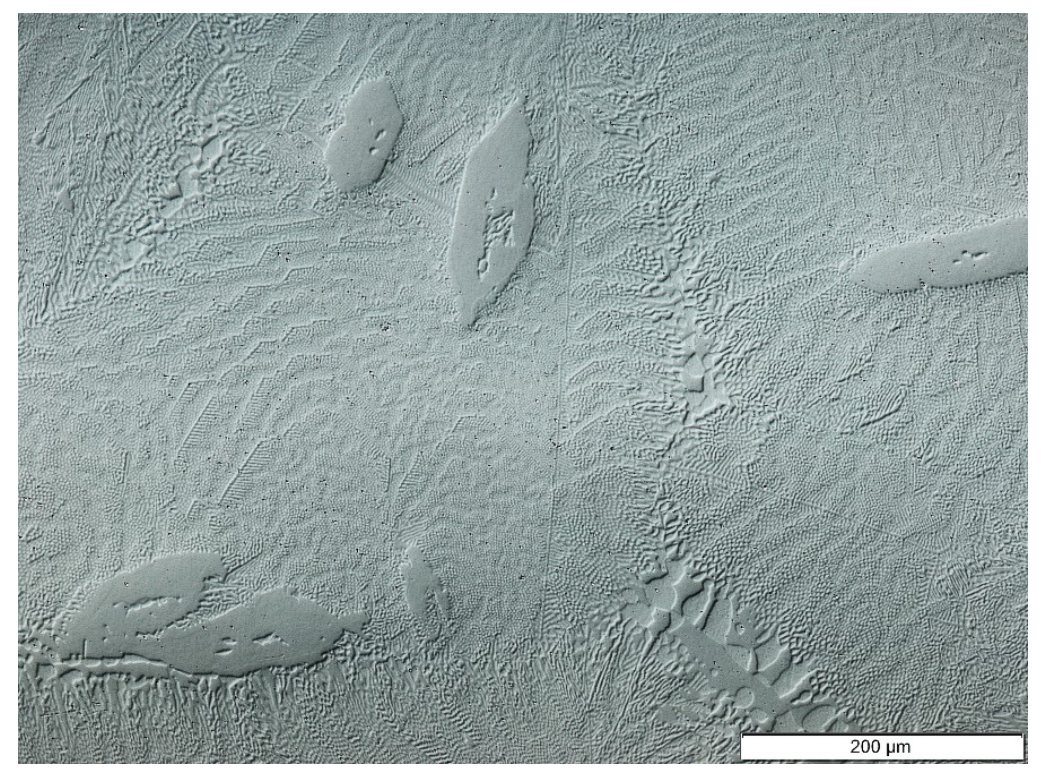

Figure 3-4: Optical microstructure of as-cast Stellite 720.

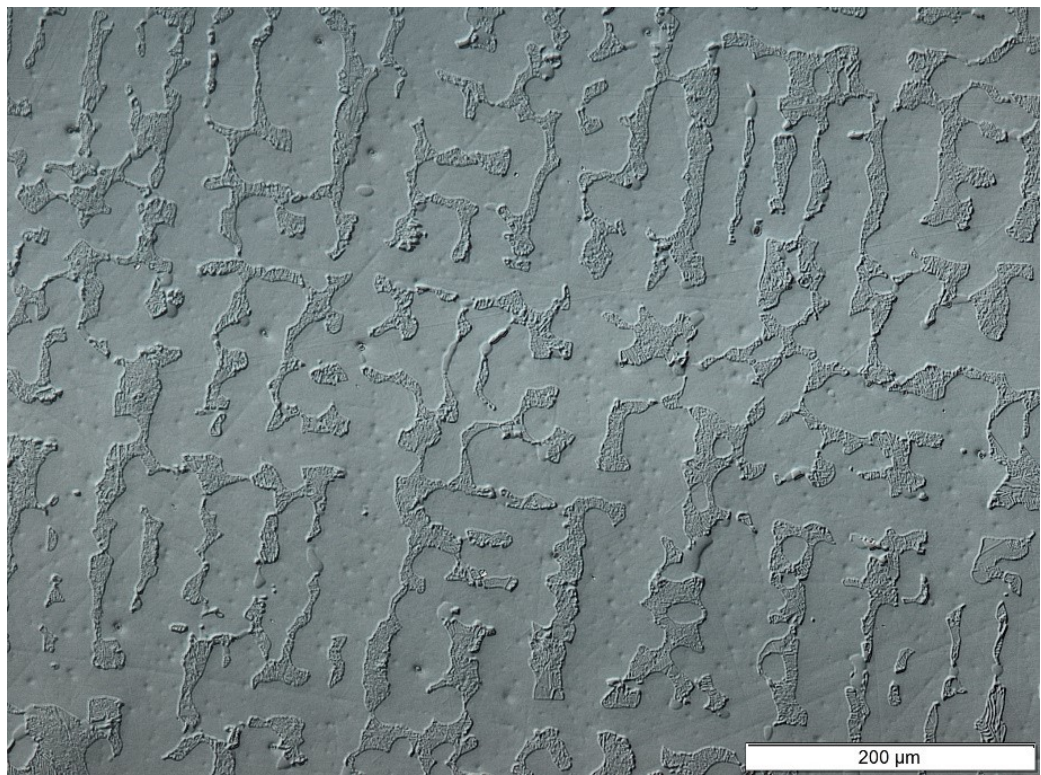

Figure 3-5: Optical microstructure of as-cast Stellite 728.

\subsubsection{SEM and EDX Analysis}

SEM analysis was complemented by EDX analysis to indicate the elements present in each phase. While EDX is a powerful tool to identify elements, the results presented are qualitative or 
semi-quantitative in nature, and indicate the elements found as an approximate wt.\%. Quantitative SEM/EDX analysis is time-consuming and approved standards are required to validate each phase to ensure accurate representation. Therefore, literature and nominal chemical composition results will be used as a guide to label the phases, with XRD being used to confirm the presence of chemical compounds in particular phases. Light elements in particular such as carbon and oxygen are in the low energy level, and are widely considered to be difficult to analyze using EDX. Elements heavier than oxygen are considered to be more measurable using EDX.

The signals of greatest interest in SEM are secondary electrons (SEs), and backscatter electrons (BSEs). SEs are the electrons that are ejected from the specimen during the incident electron beam acting on the specimen, and escape the sample surface and provide greater resolution for imaging. BSEs are the electrons from the incident beam that re-emerge from deeper in the material, and they related to atomic number or composition of the material. The SEM images taken using BSEs clearly show the carbide morphology due to the different composition of the phases.

\subsubsection{SEM/EDX Analysis of Stellite 21}

Stellite 21 by virtue of its low carbon content, has a low volume fraction of carbides. Figure 3-6 shows a high magnification view of the lamellar eutectic using BSEs. Figure 3-7a shows individual EDX points taken in the solid solution and lamellar eutectic. A couple of points were chosen within the lamellar eutectic to ascertain if a qualitative difference between the fineness of the eutectic existed. However, both the finer and the relatively coarser eutectic did not show a qualitative difference Figure 3-7c and Figure 3-7d. The lamellar eutectic was found to contain primarily $\mathrm{Cr}$, Co, Mo and $\mathrm{C}$, and a lesser extent of Ni, Si, W and Fe. The EDX map shown in 
Figure 3-8 reveals that both $\mathrm{Cr}$ and Mo have the strongest signature. An EDX map is generated via the number of counts, or signals, being emitted from a particular area. Therefore, a stronger signature will be deeper in colour. This information shows that a CrMo-rich carbide and Mo-rich intermetallic or carbides can be present.

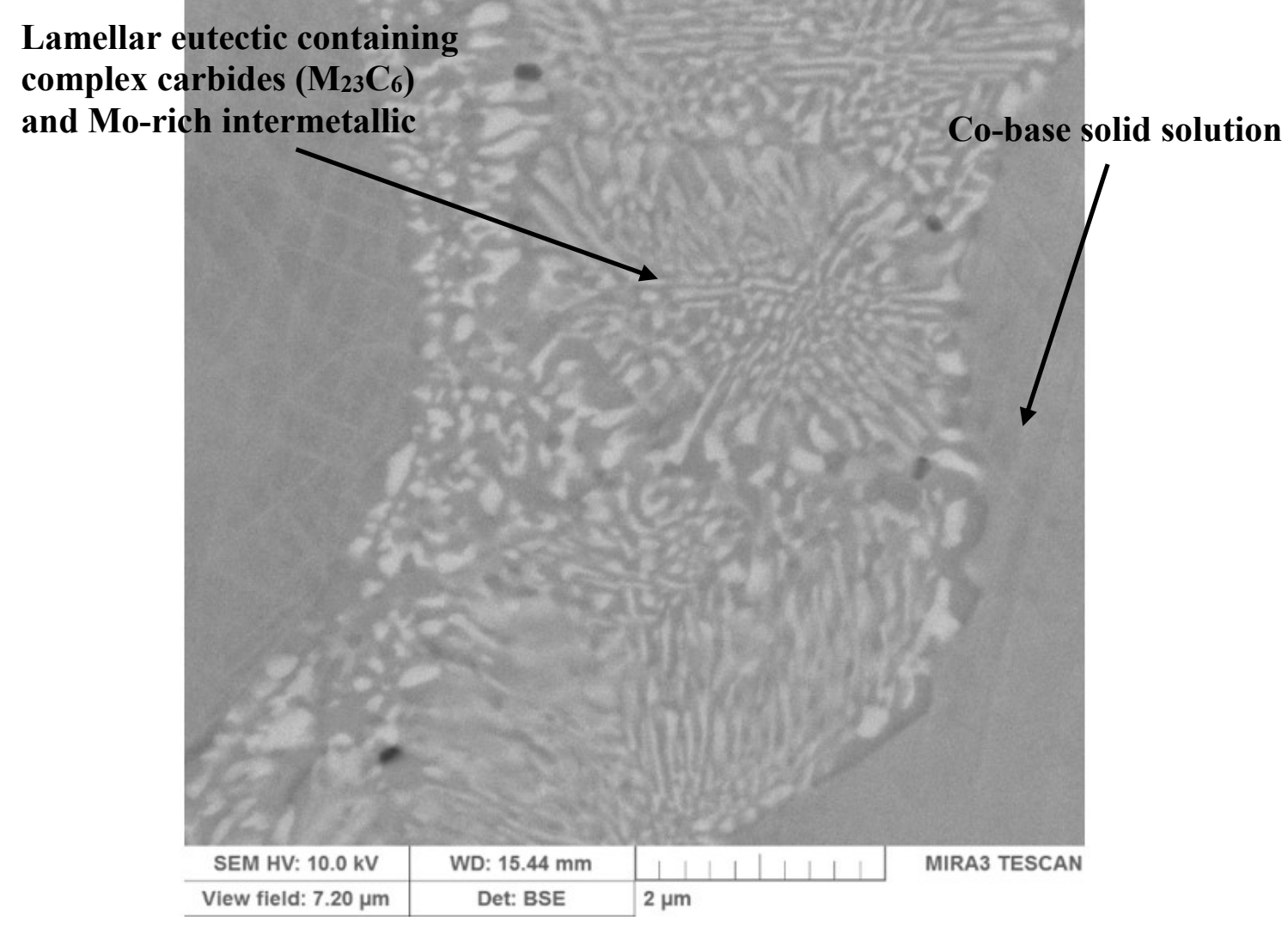

Figure 3-6: Detail of Stellite 21 microstructure using BSEs. 


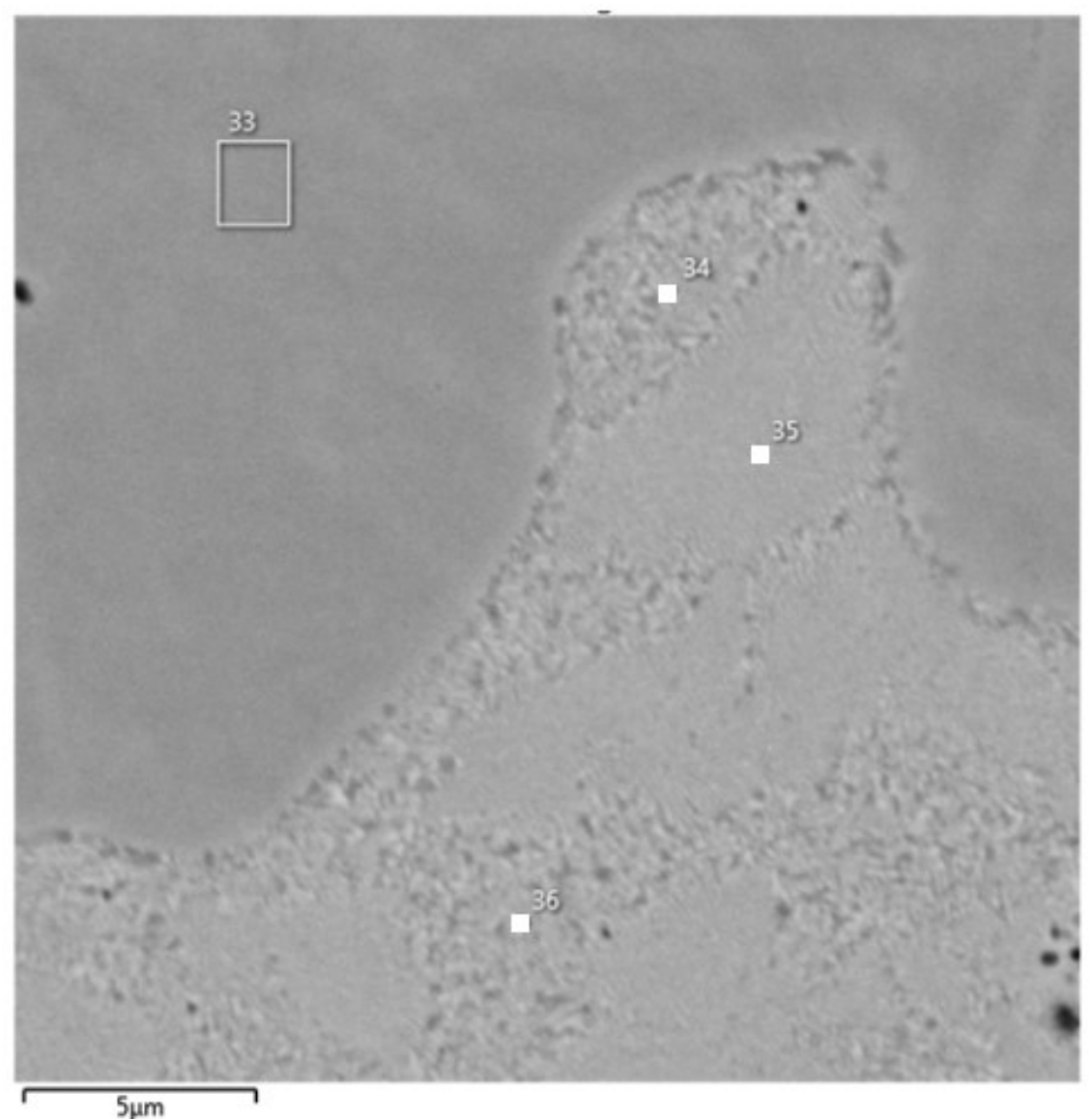

(a)

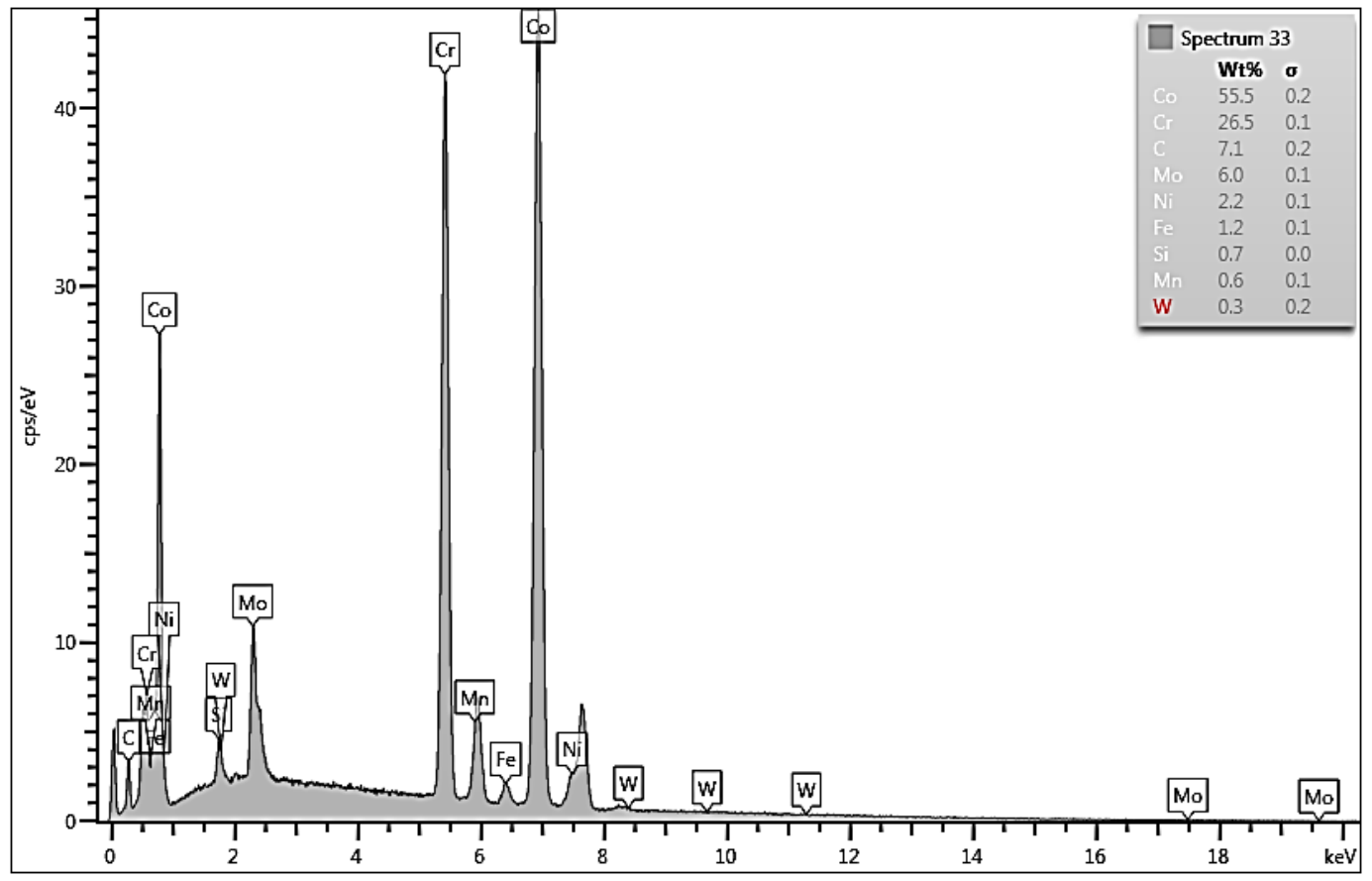

(b) 


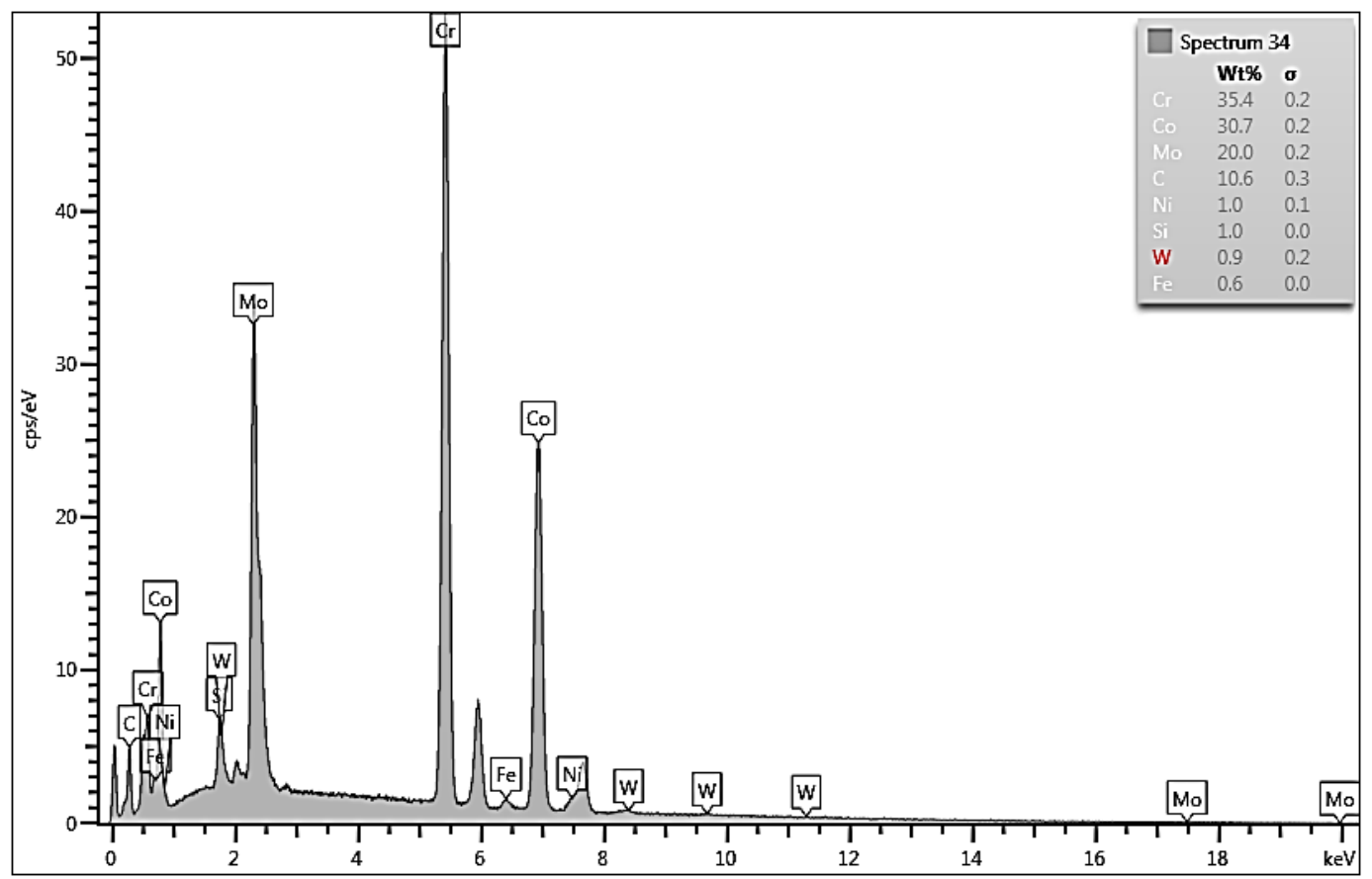

(c)

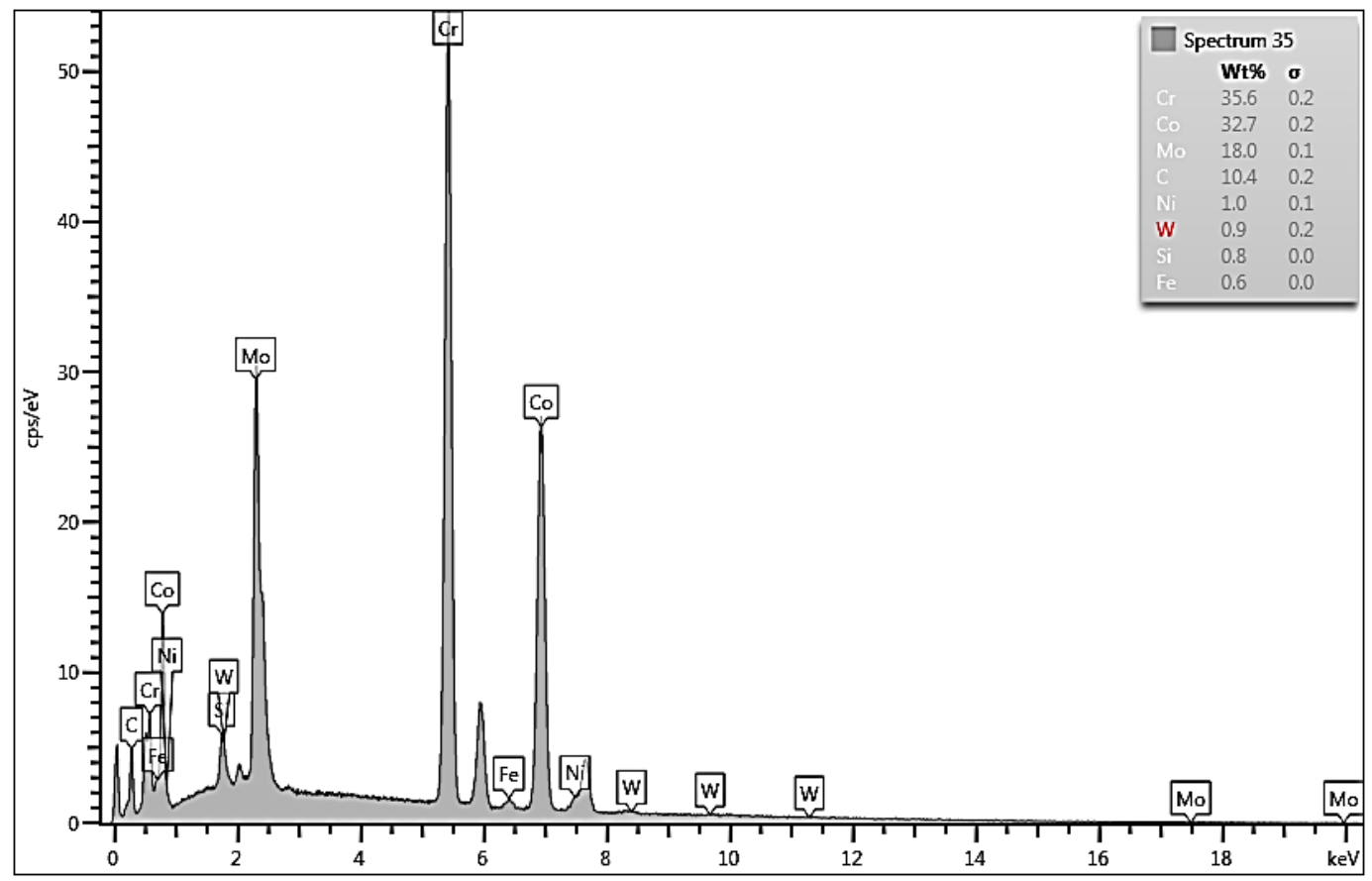

(d)

Figure 3-7: EDX analysis of as-cast Stellite 21 microstructure (a) locations for point analysis, (b) spectrum \#33 dark grey (solid solution), (c) spectrum \#34 coarser eutectic carbide area, (d) spectrum $\# 35$ finer eutectic carbide area. 


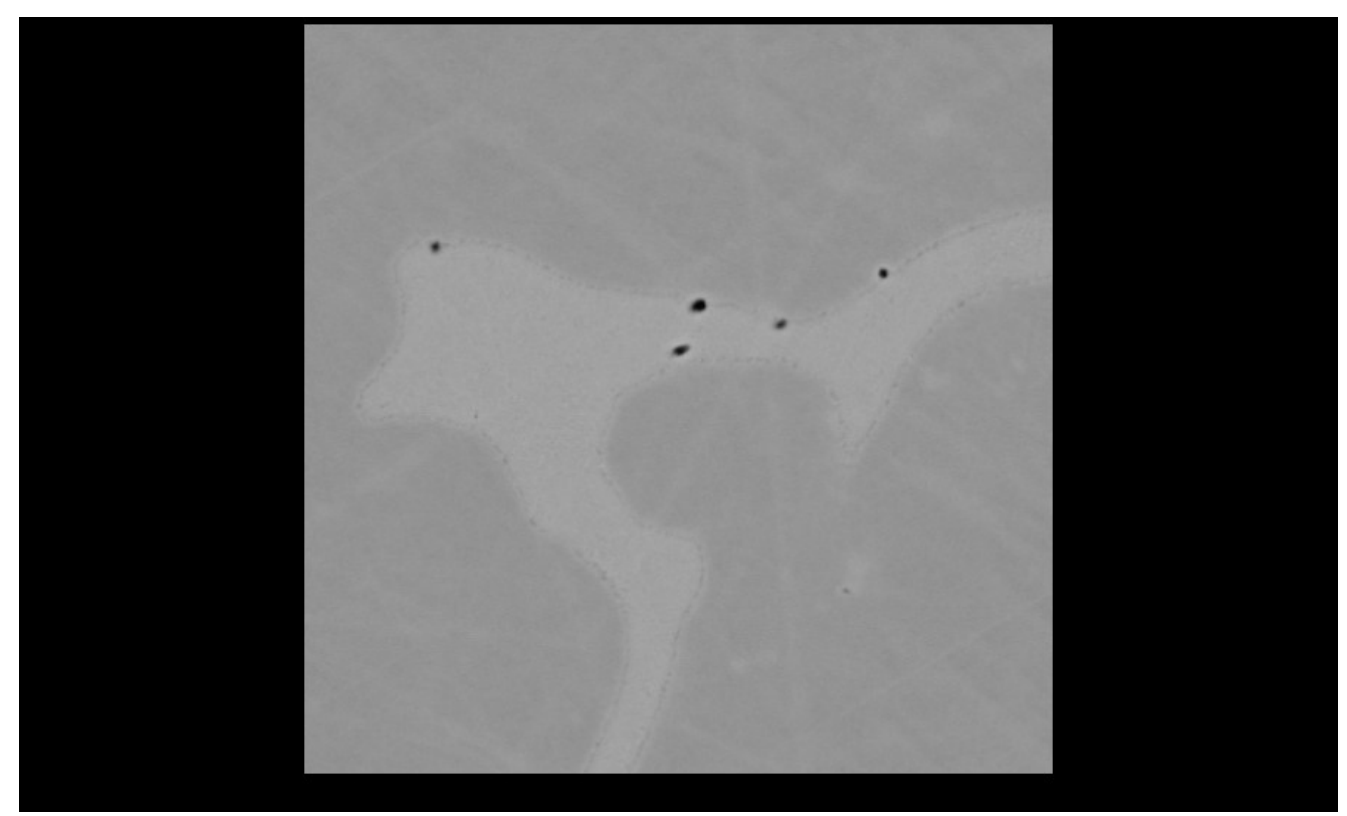

(a)
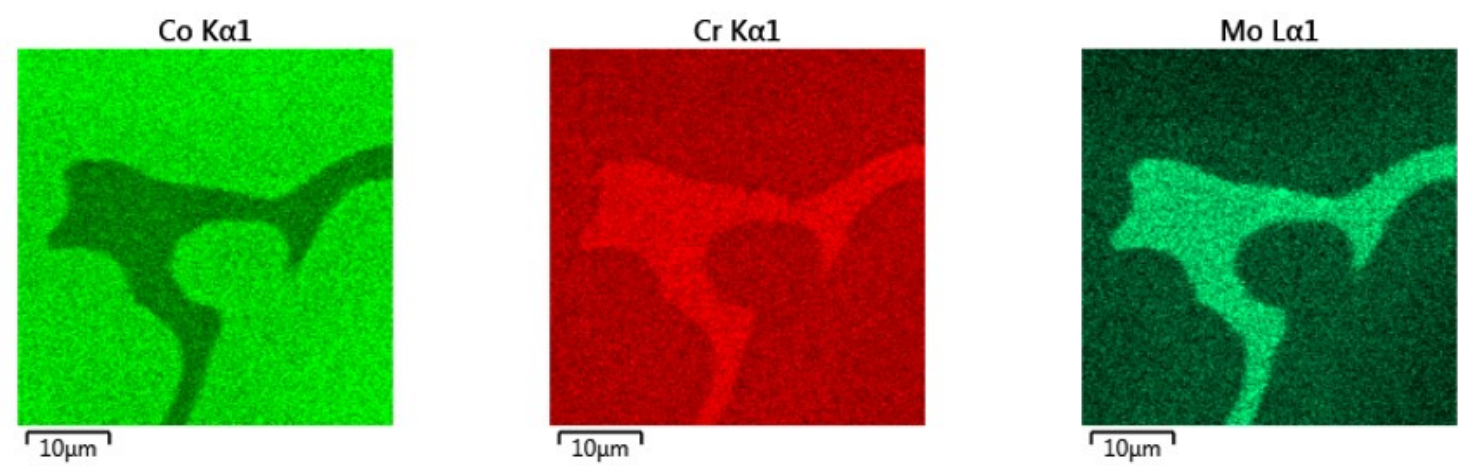

Ni $K \alpha 1$

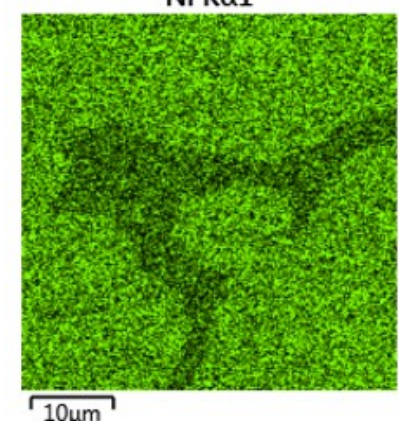

Si $K \alpha 1$

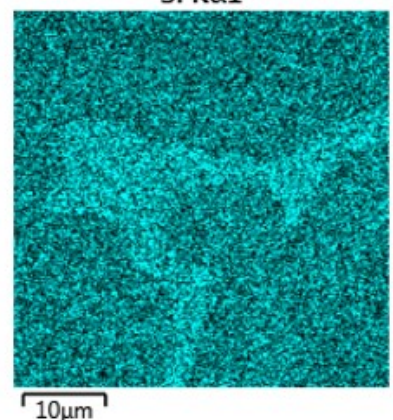

C Ka1_2

(b)

Figure 3-8: EDX map of as-cast Stellite 21 carbide phase (a) image used for EDX map, (b) individual element maps. 


\subsubsection{SEM/EDX Analysis of Stellite 720}

Stellite 720 shows a very compact microstructure of what appears to be four distinct phases, as shown in Figure 3-9. It consists of large carbides which are hexagonal, the surrounding dendritic shaped carbides and the solid solution matrix. The EDX analysis of individual phases via a point analysis is shown in Figure 3-10. Although qualitative, the detection of elements using EDX helps establish the type of phases that could be present. The large hexagonal shaped carbide (dark grey) consists of a Cr-rich carbide phase, with lesser amounts of Mo and Co also being detected. The medium grey phase primarily consists of $\mathrm{Cr}$, $\mathrm{Co}$, Mo and $\mathrm{C}$, which is expected to be a Cr-rich carbide, and/or CrCoMo complex carbide. The light grey phase showed numerous elements in the point analysis and is therefore expected to form the solid solution. In addition, the EDX map shown in (Figure 3-11) reveals a strong Co signature in this phase, suggesting a Co solid-solution. The same EDX map shows the distinctive outline of Mo, Co, and $\mathrm{Si}$ in the white phase, suggesting intermetallics are present in this phase.

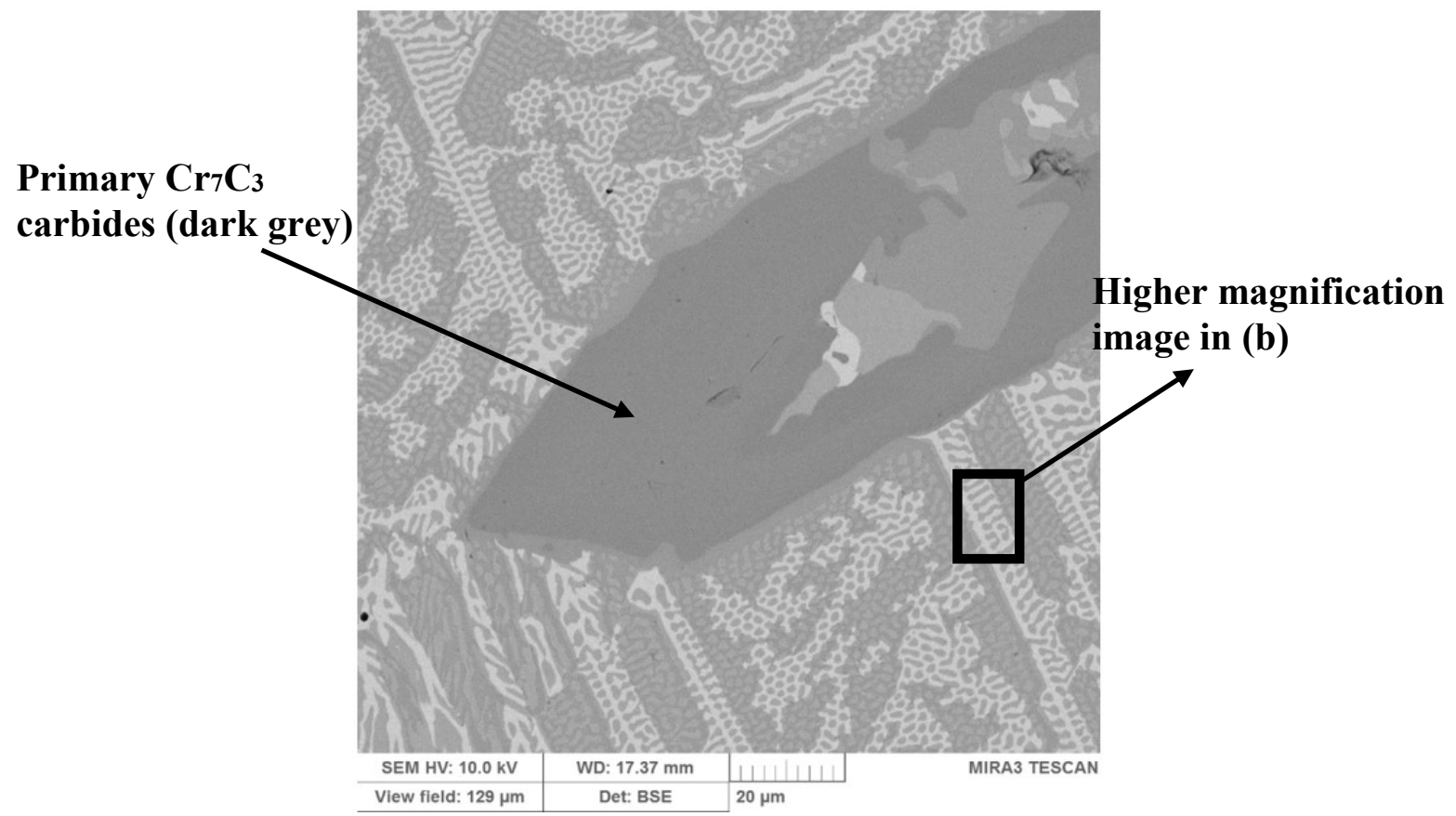

(a) 


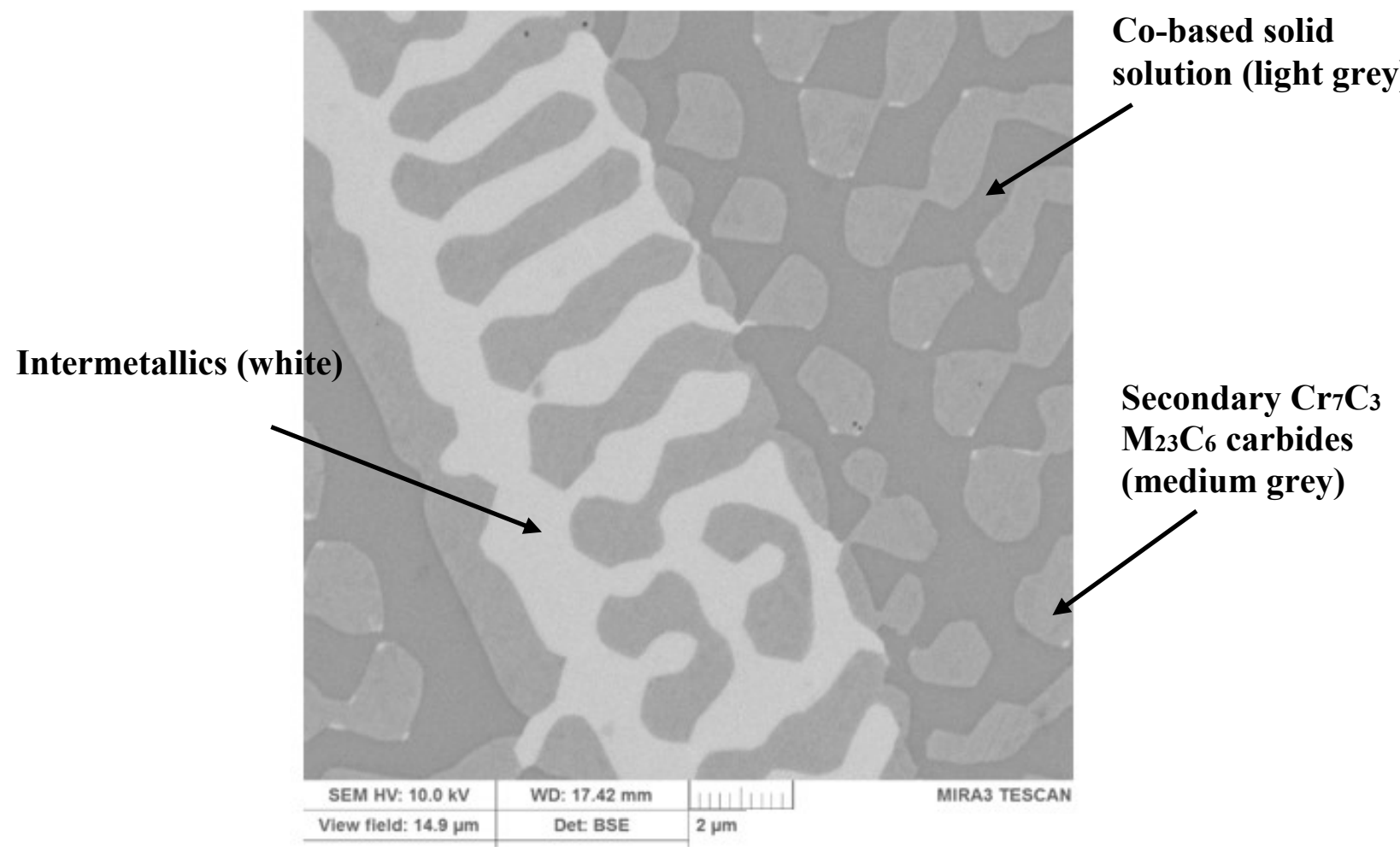

(b)

Figure 3-9: SEM microstructure of Stellite 720 using BSE image (a) lower magnification, (b) higher magnification. 


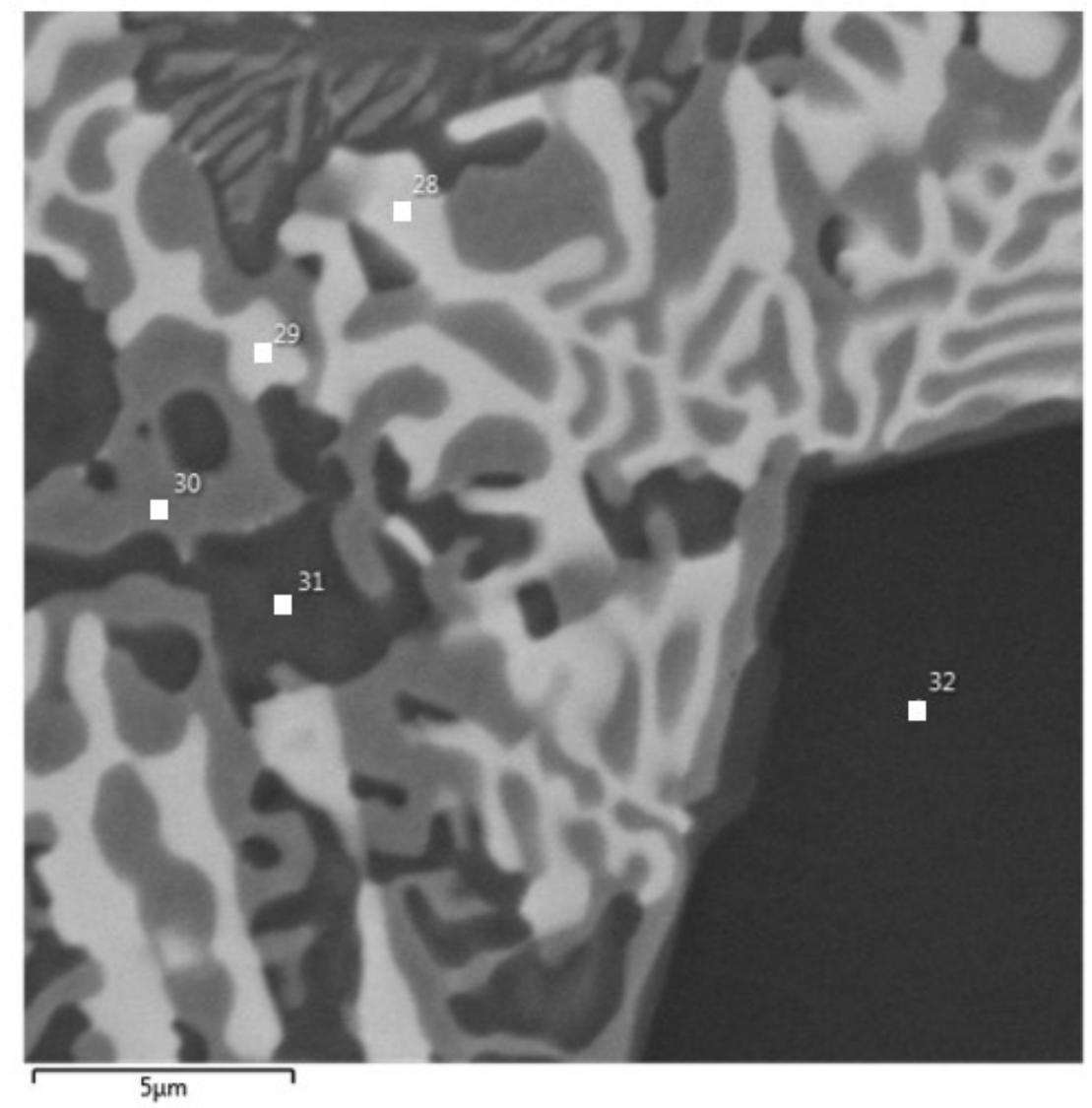

(a)

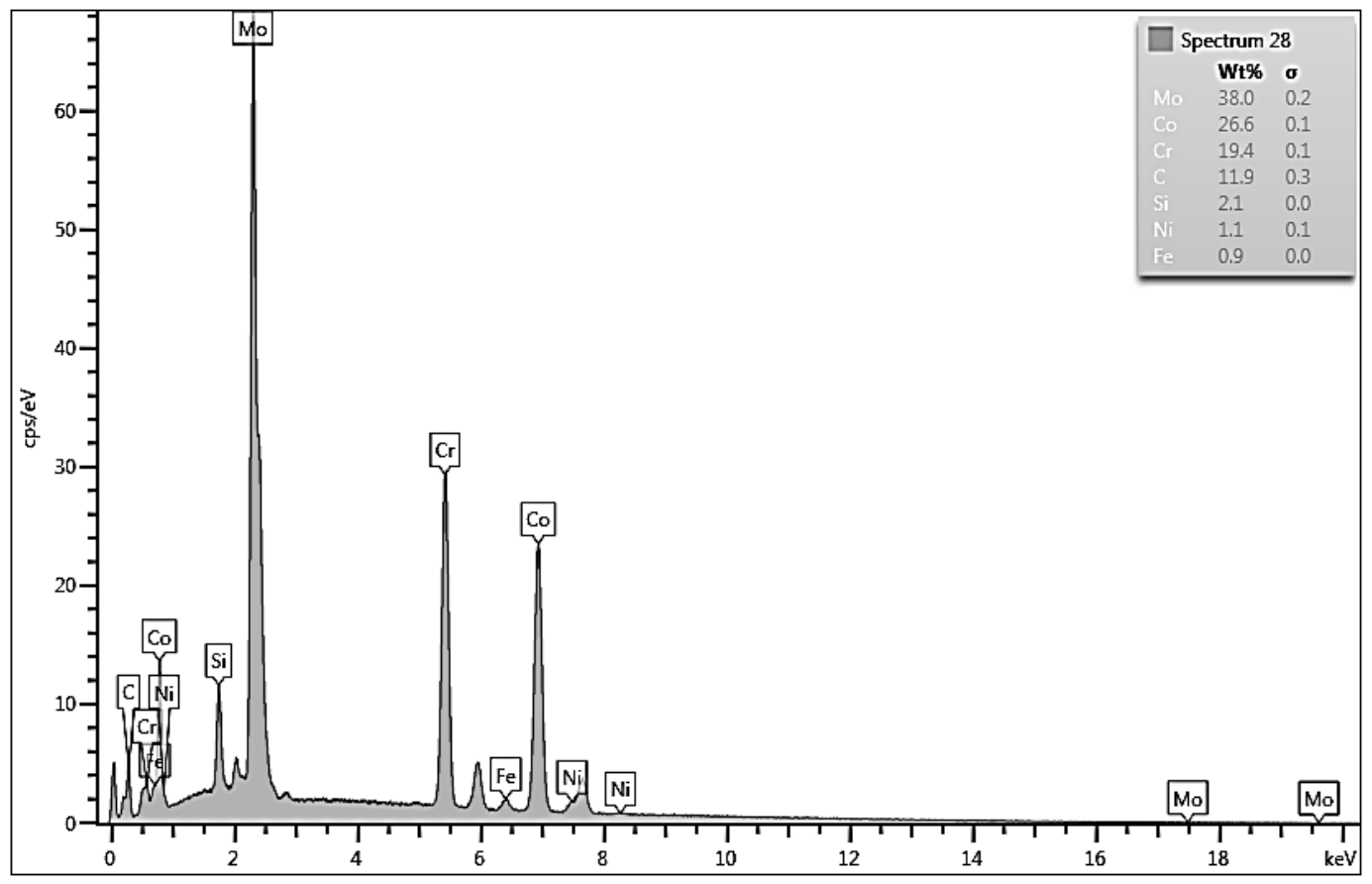

(b) 


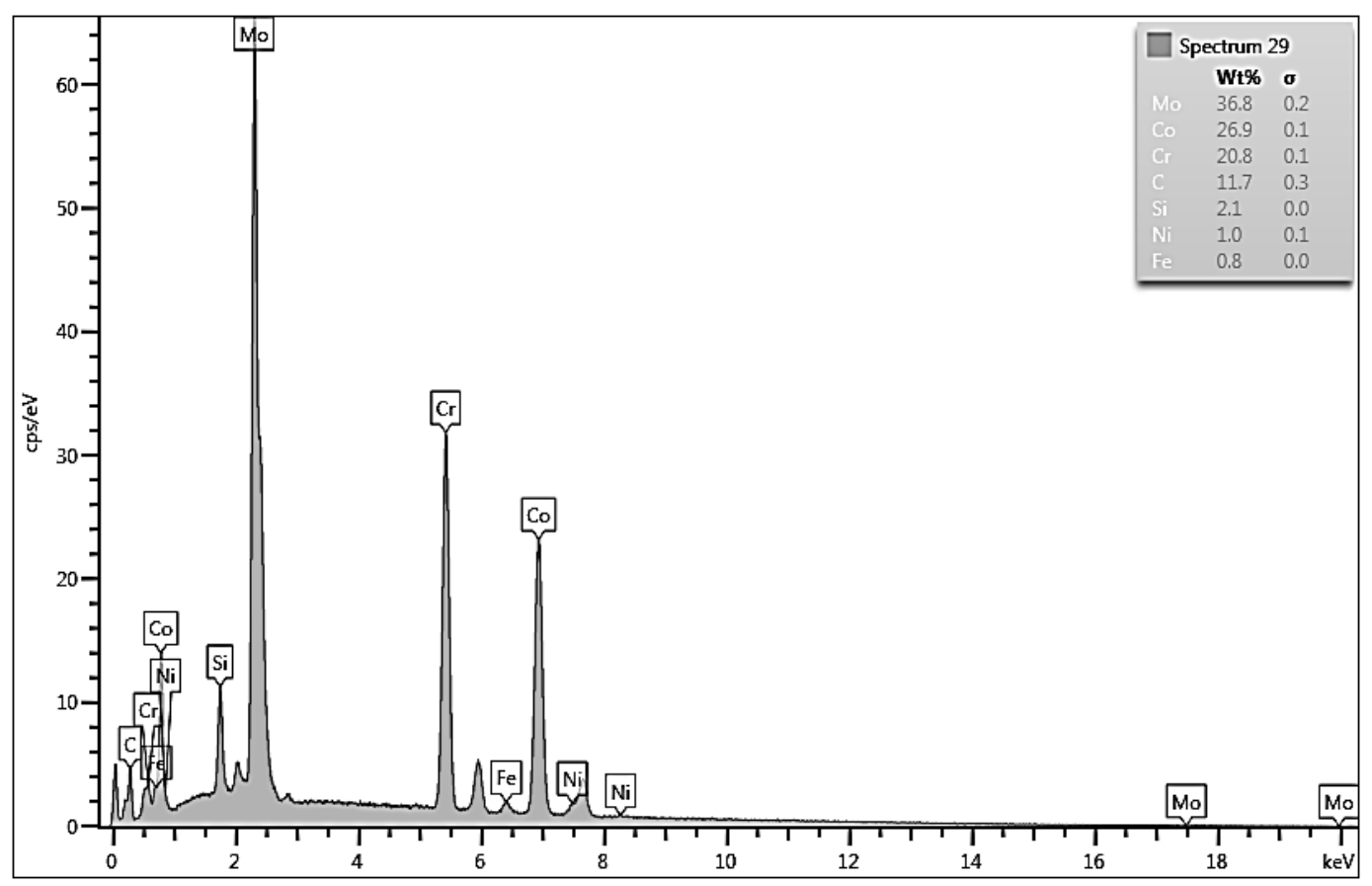

(c)

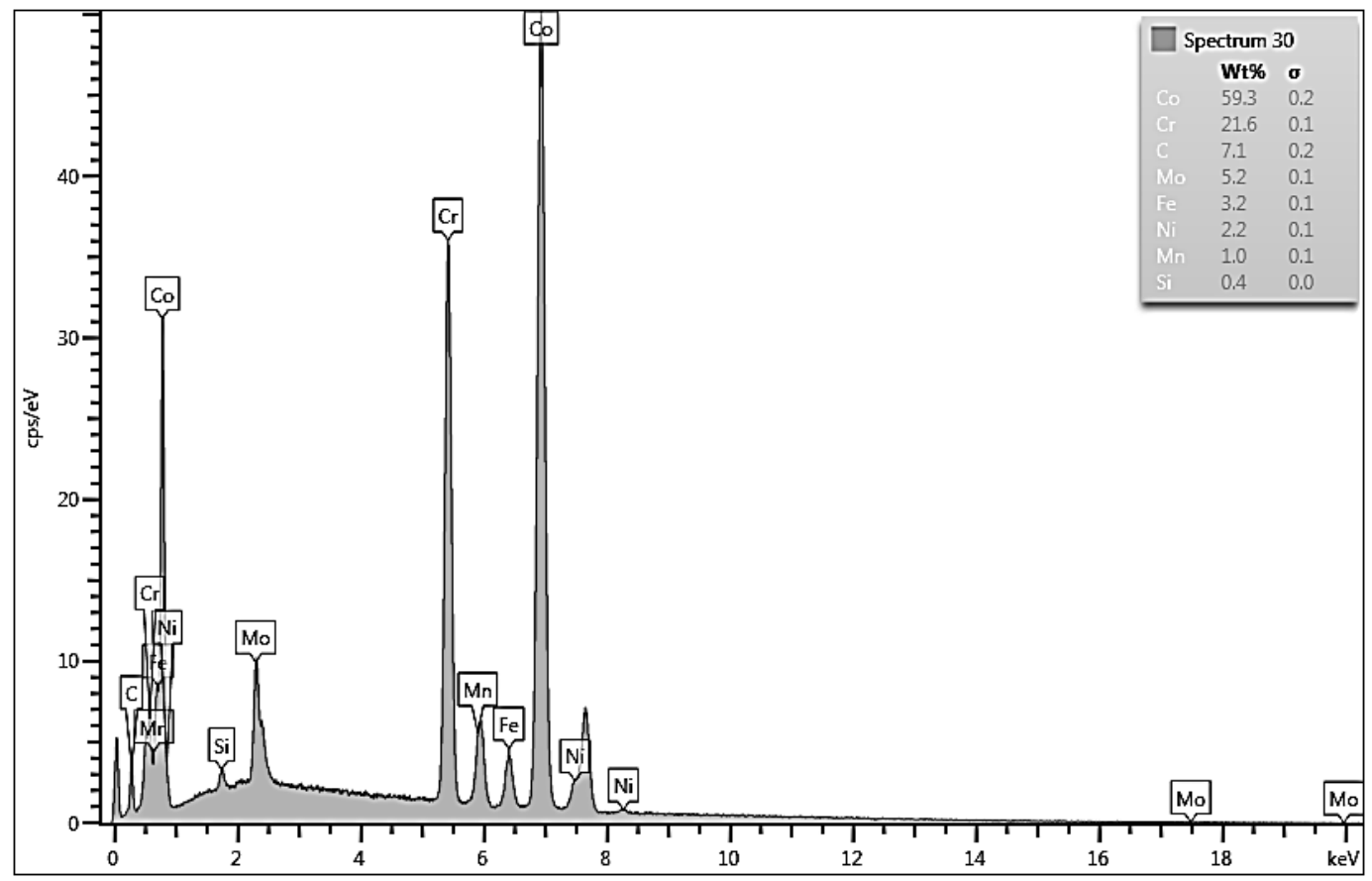

(d) 


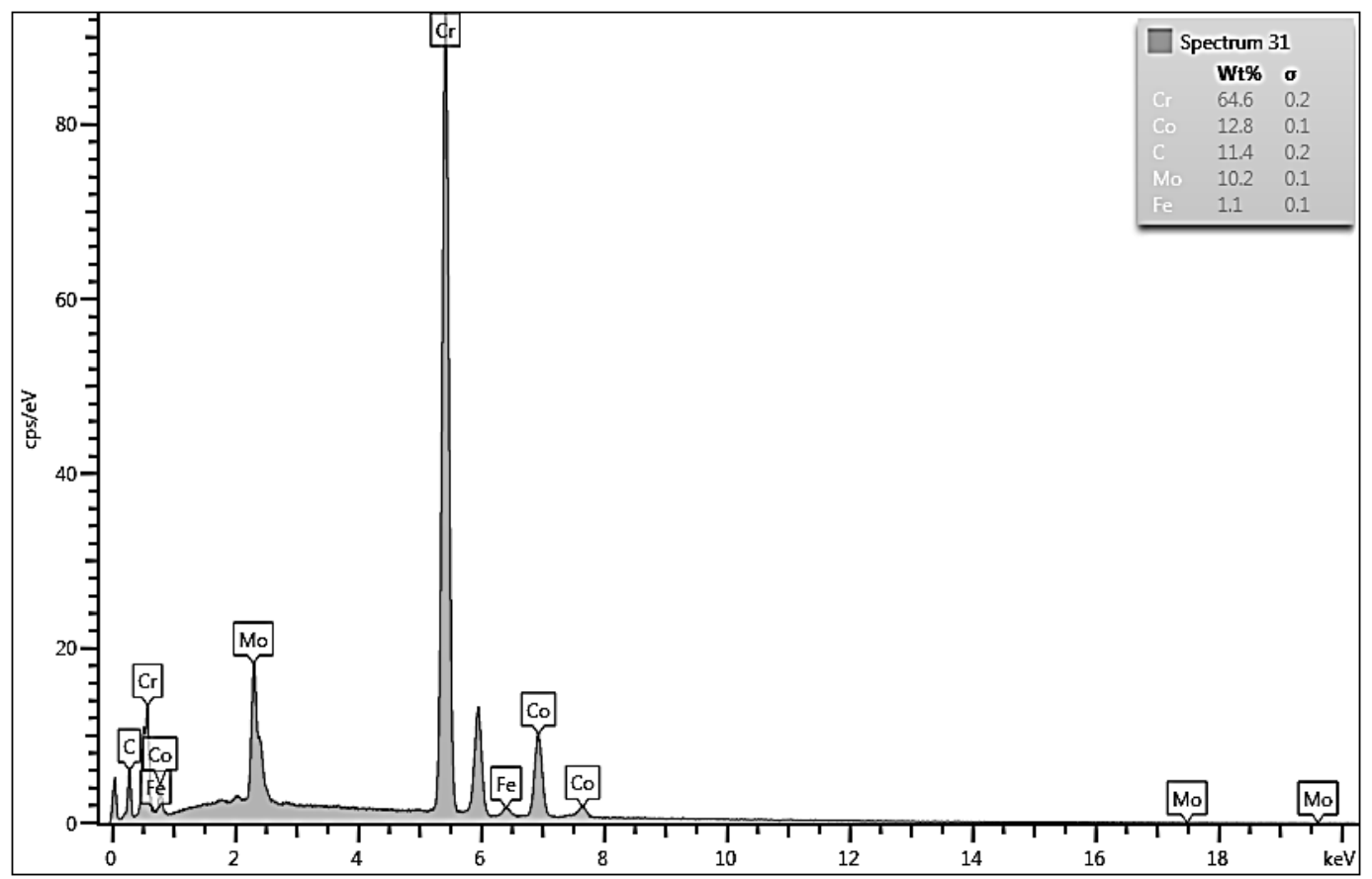

(e)

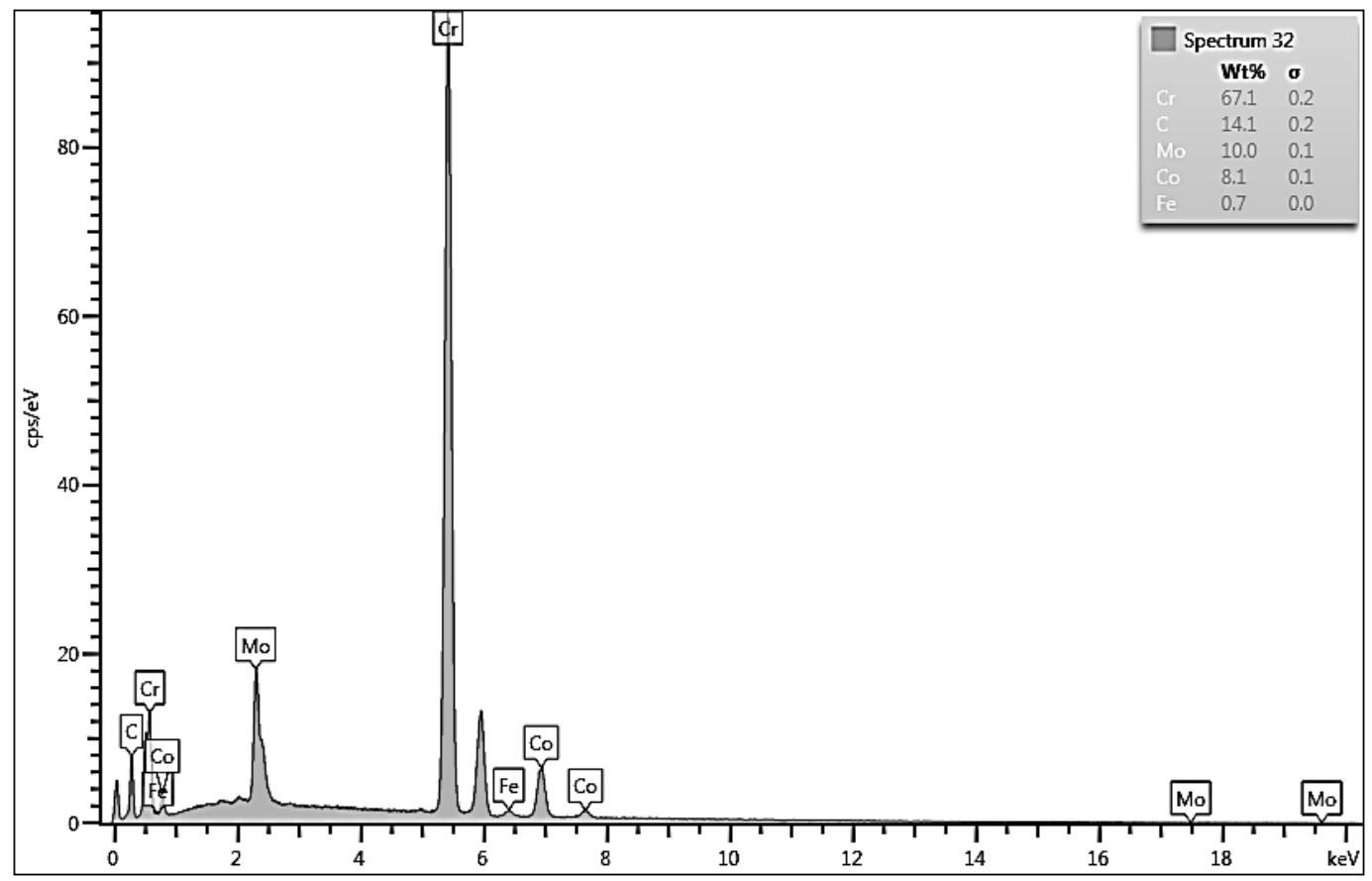

(f)

Figure 3-10: EDX analysis of as-cast Stellite 720 (a) locations for point analysis, (b) spectrum \#28 white phase, (c) spectrum \#29 white phase (second location), (d) spectrum \#30 light grey, (e) spectrum \#31 medium grey, (f) spectrum \#32 dark grey. 


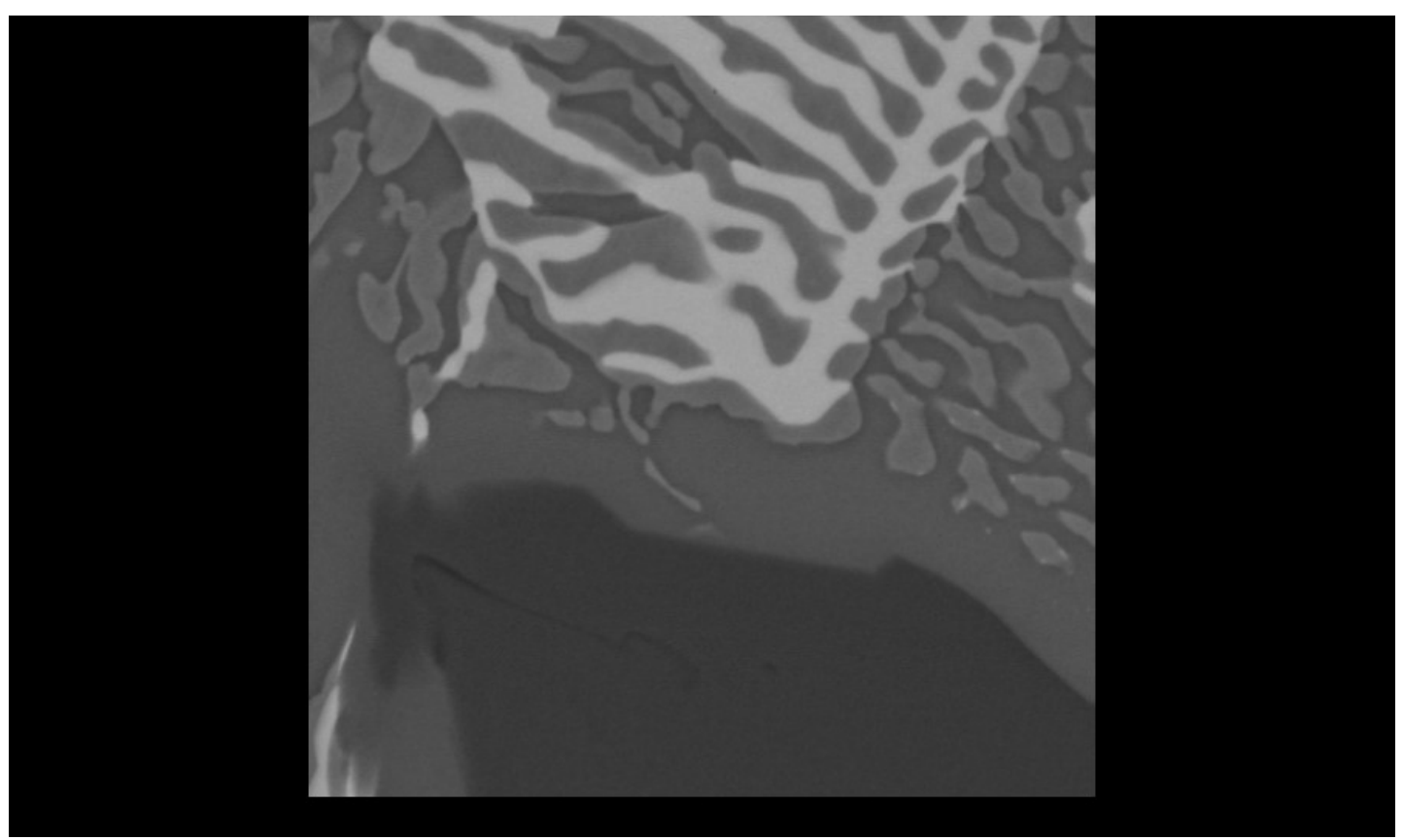

(a)
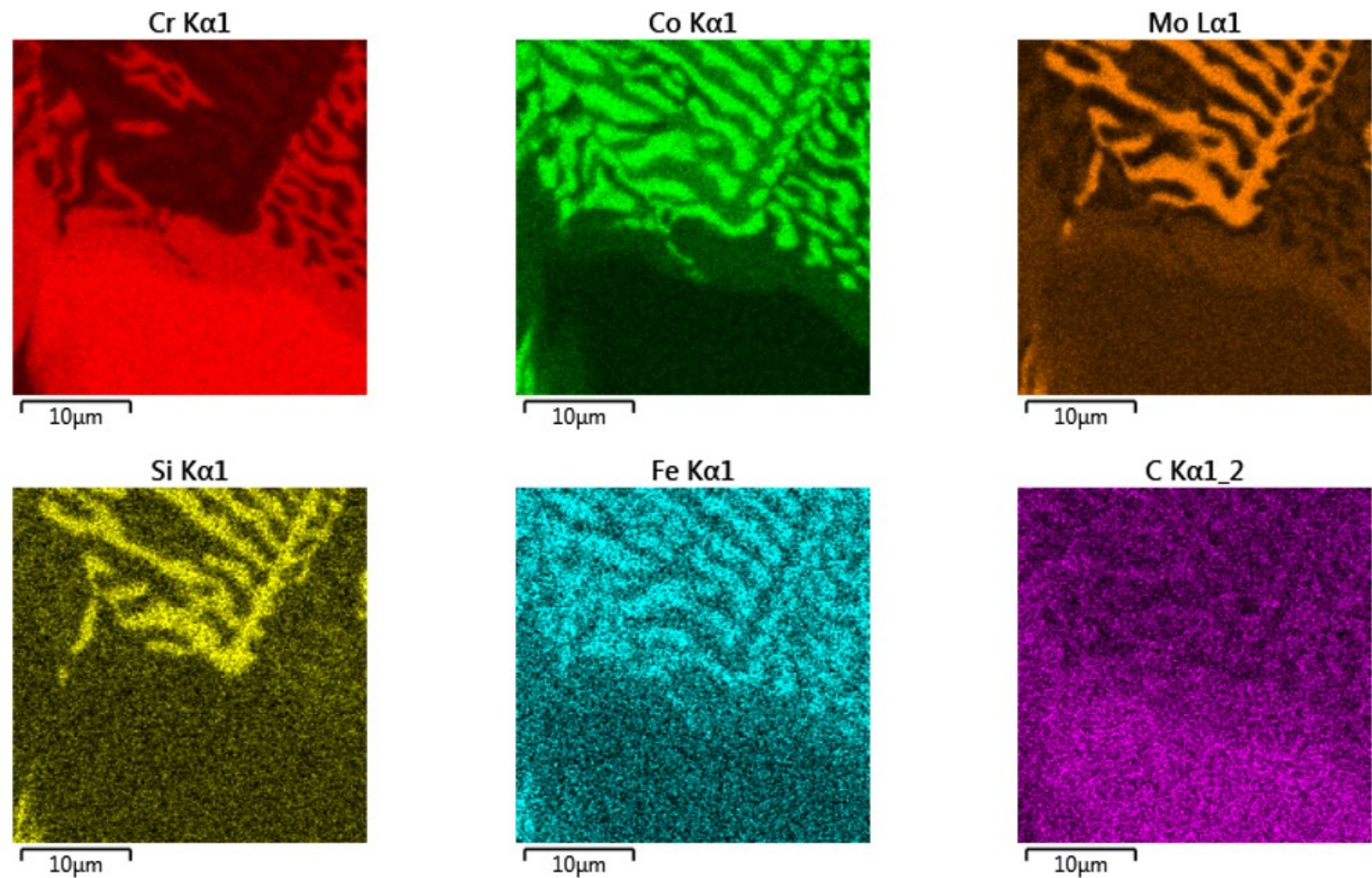

(b)

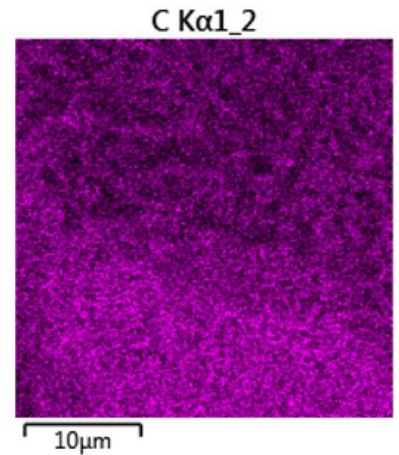

Figure 3-11: EDX map of as-cast Stellite 720 (a) EDX map image, (b) individual element maps. 


\subsubsection{SEM/EDX Analysis of Stellite 728}

Stellite 728 shows a typical patterned microstructure with three distinct phases, as shown in Figure 3-12. It consists of a white dendritic phase with a larger light grey particle ingrained, both of which sit in a dark grey matrix. The EDX analysis of the individual phases via point analysis is shown in Figure 3-13. The dark grey phase, identified as the solid solution, consists of $\mathrm{Co}, \mathrm{Cr}, \mathrm{Mo}, \mathrm{C}, \mathrm{Ni}, \mathrm{Si} \mathrm{Fe}, \mathrm{Mn}$ and $\mathrm{Nb}$. This phase is also present between the white phase shown in Figure 3-13a as point 40. The light grey phase shows numerous elements in the point analysis including $\mathrm{Co}, \mathrm{Cr}$, Mo $\mathrm{C}$ and $\mathrm{Nb}$. This phase is expected to be a mixture of complex carbides of the $\mathrm{M}_{23} \mathrm{C}_{6}$ type, with complex $\mathrm{Nb}$ carbides forming a small part of the phase. Mo and $\mathrm{Nb}$ have the strongest signature in the white phase when viewing the EDX map (Figure 3-14). This suggests that complex $\mathrm{Nb}$ carbides and complex intermetallics are present in the form of $\mathrm{Co}_{3} \mathrm{Mo}$.

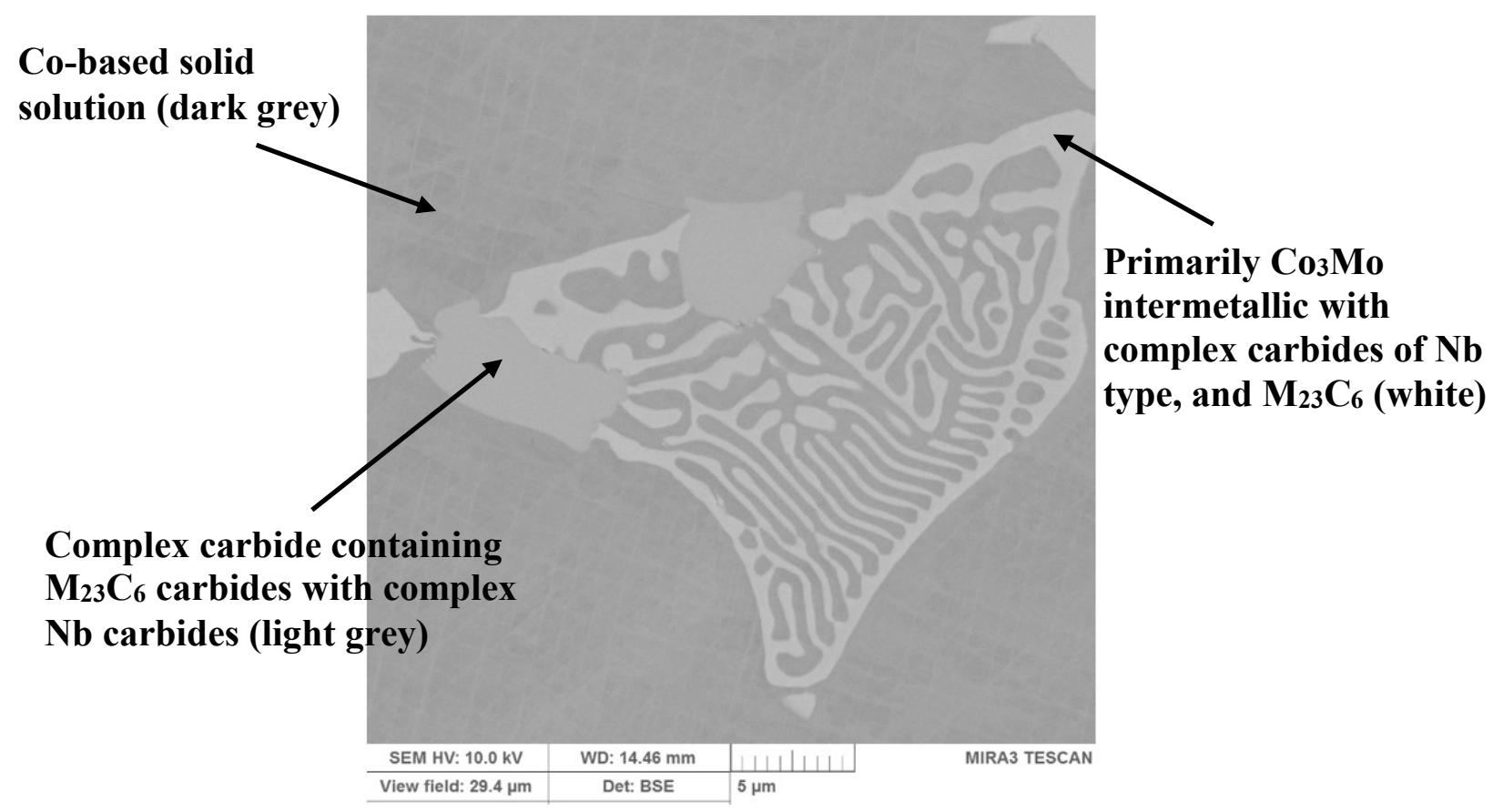

Figure 3-12: SEM microstructure of Stellite 728 using BSE image. 


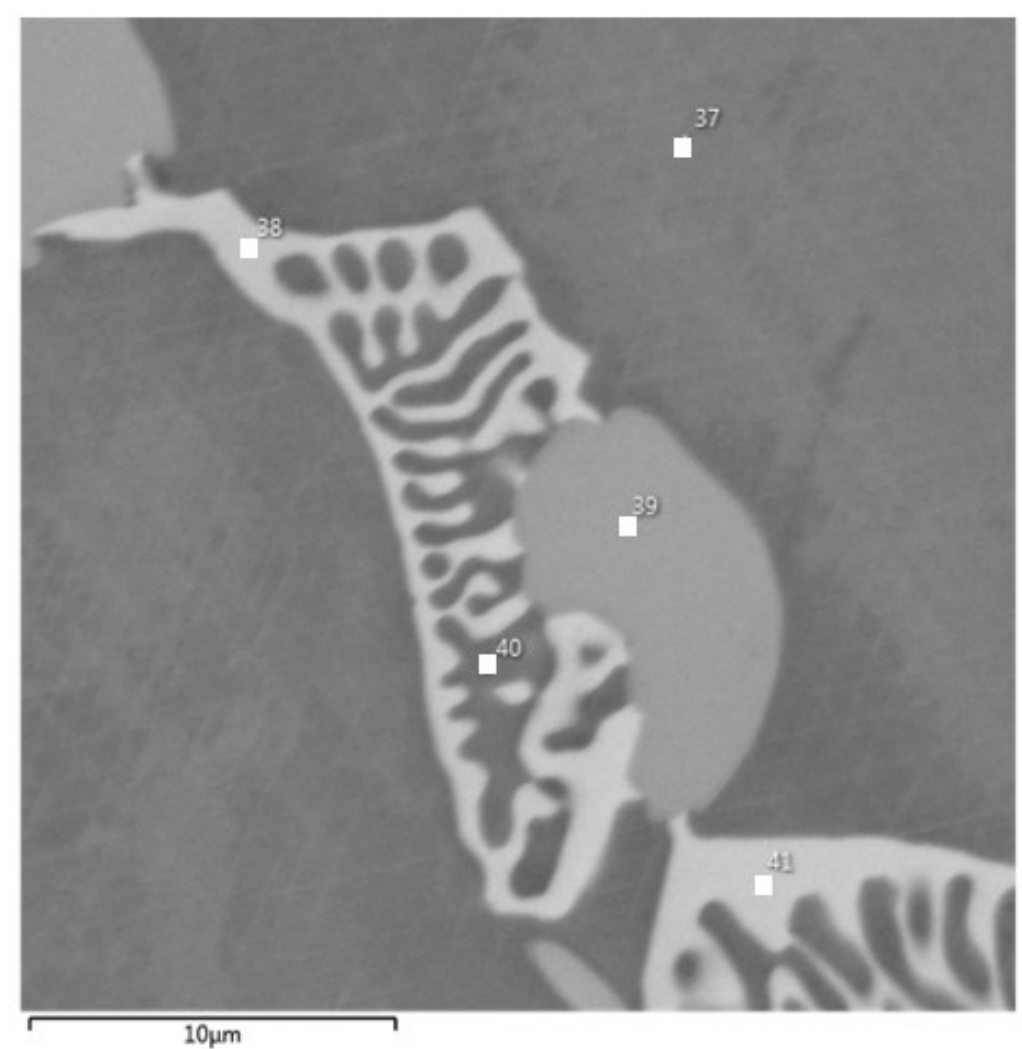

(a)

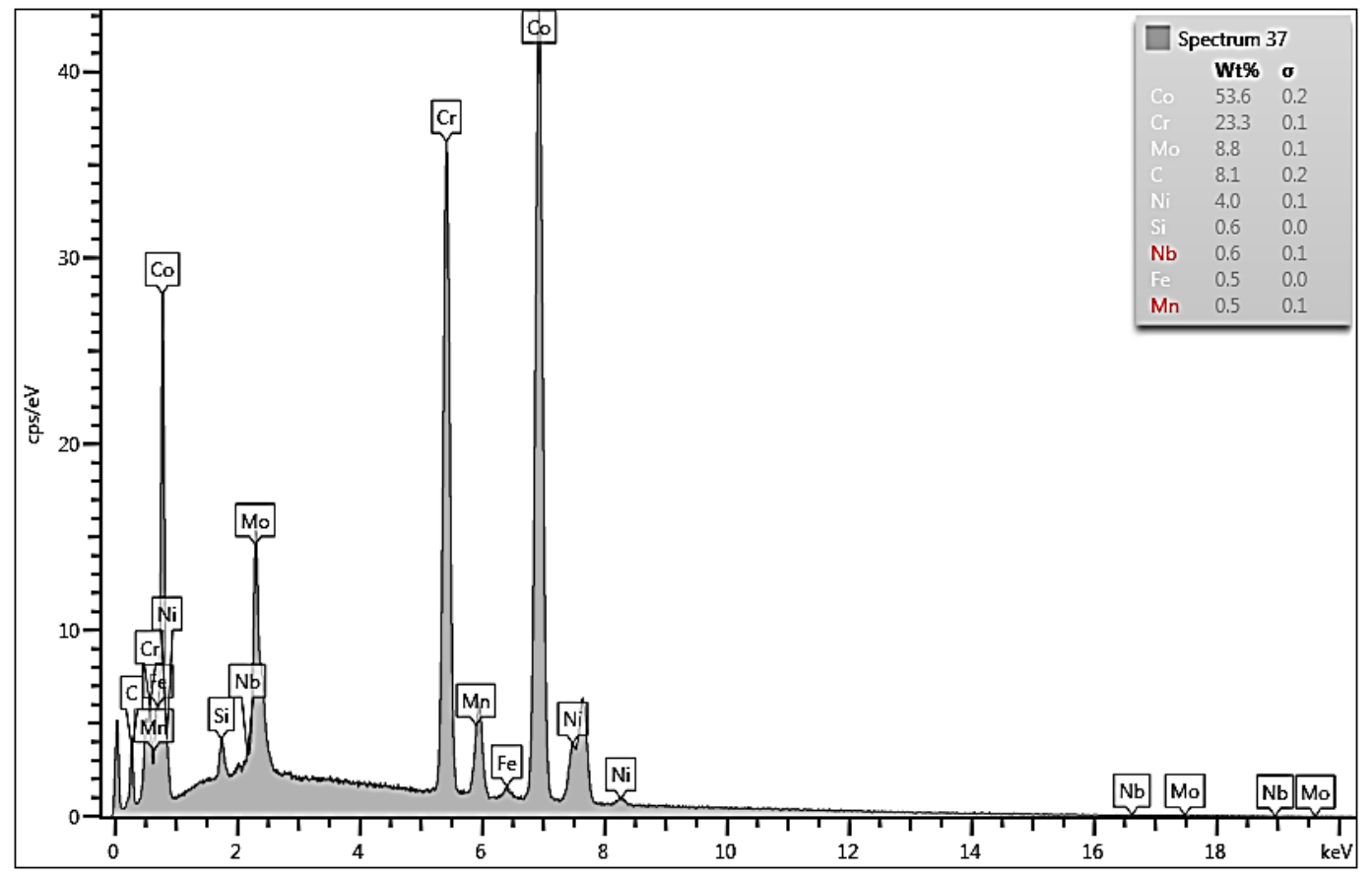

(b) 


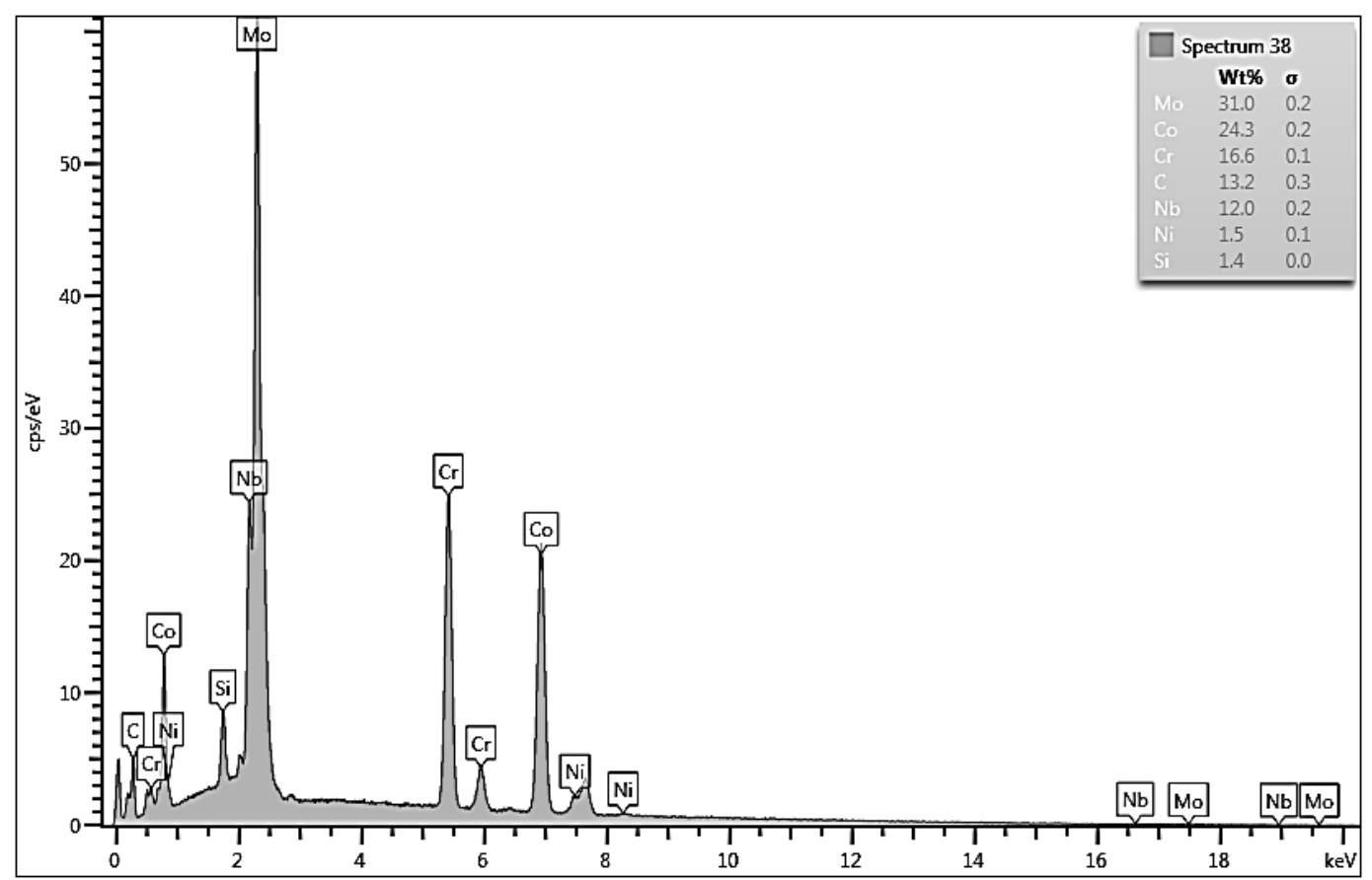

(c)

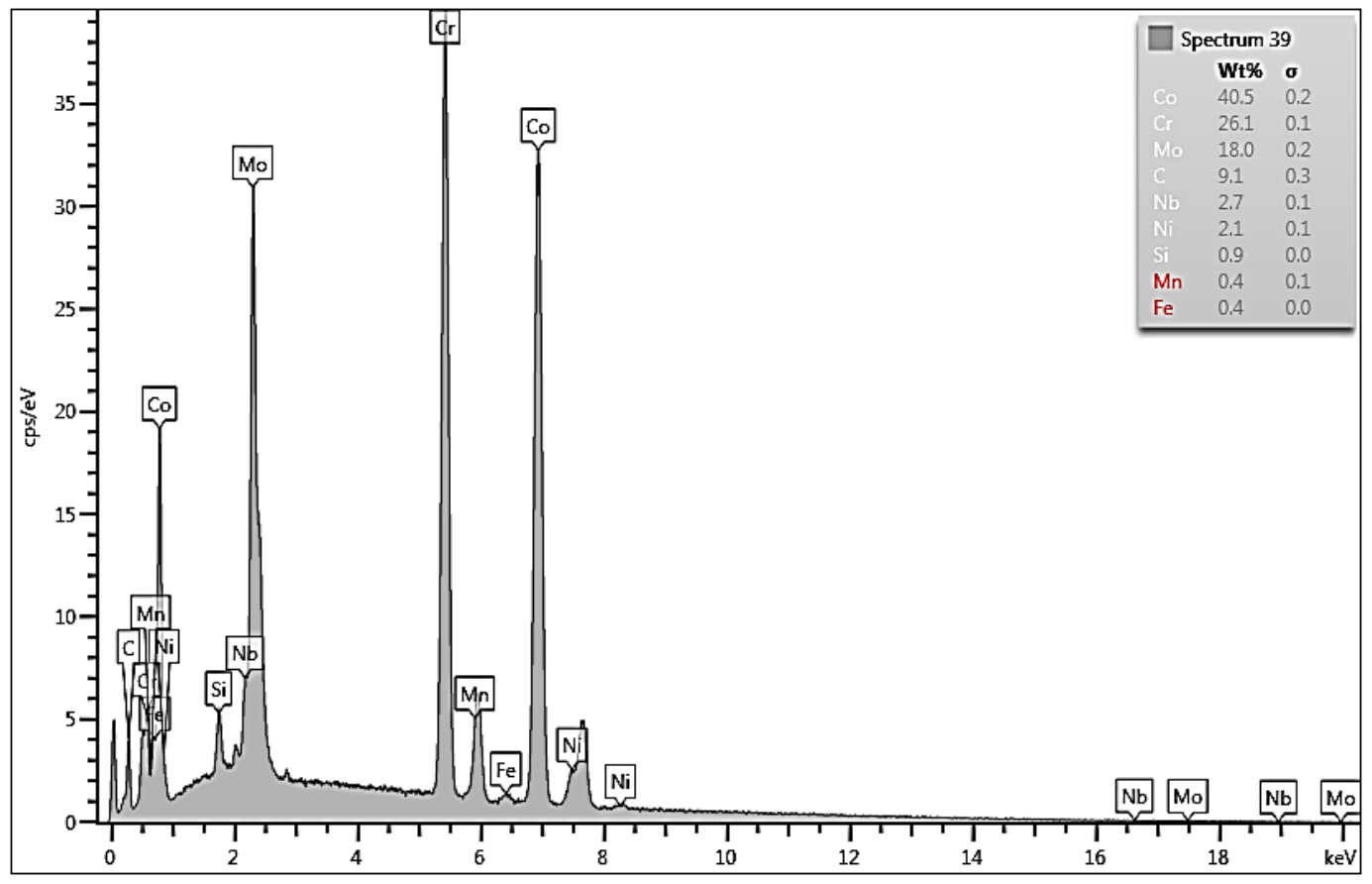

(d) 


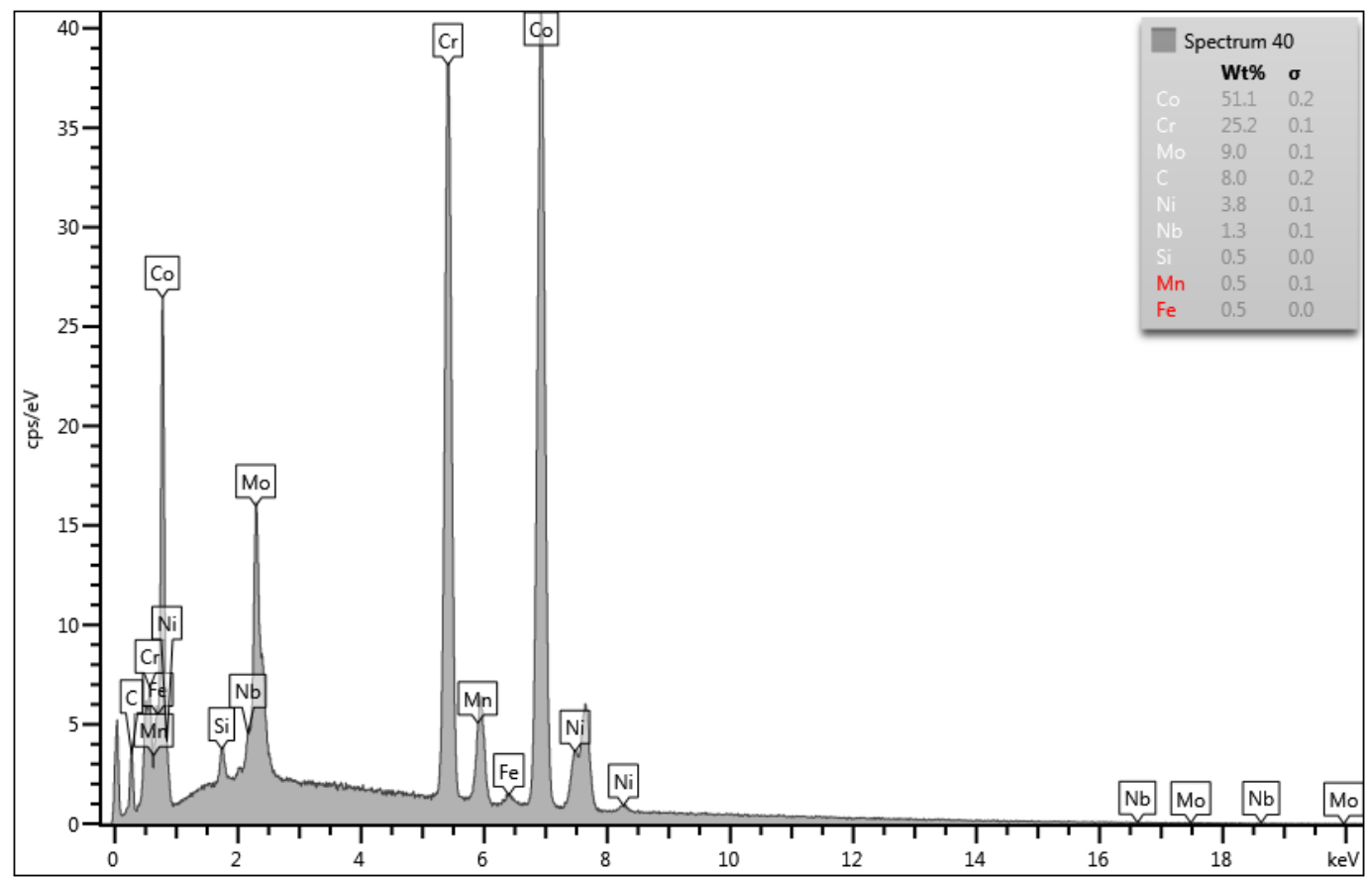

(e)

Figure 3-13: EDX analysis of as-cast Stellite 728 microstructure (a) locations for point analysis, (b) spectrum \#37 dark grey (solid solution), (c) spectrum \#38 white phase, (d) spectrum \#39 light grey, (e) spectrum \#40 dark grey (solid solution). 


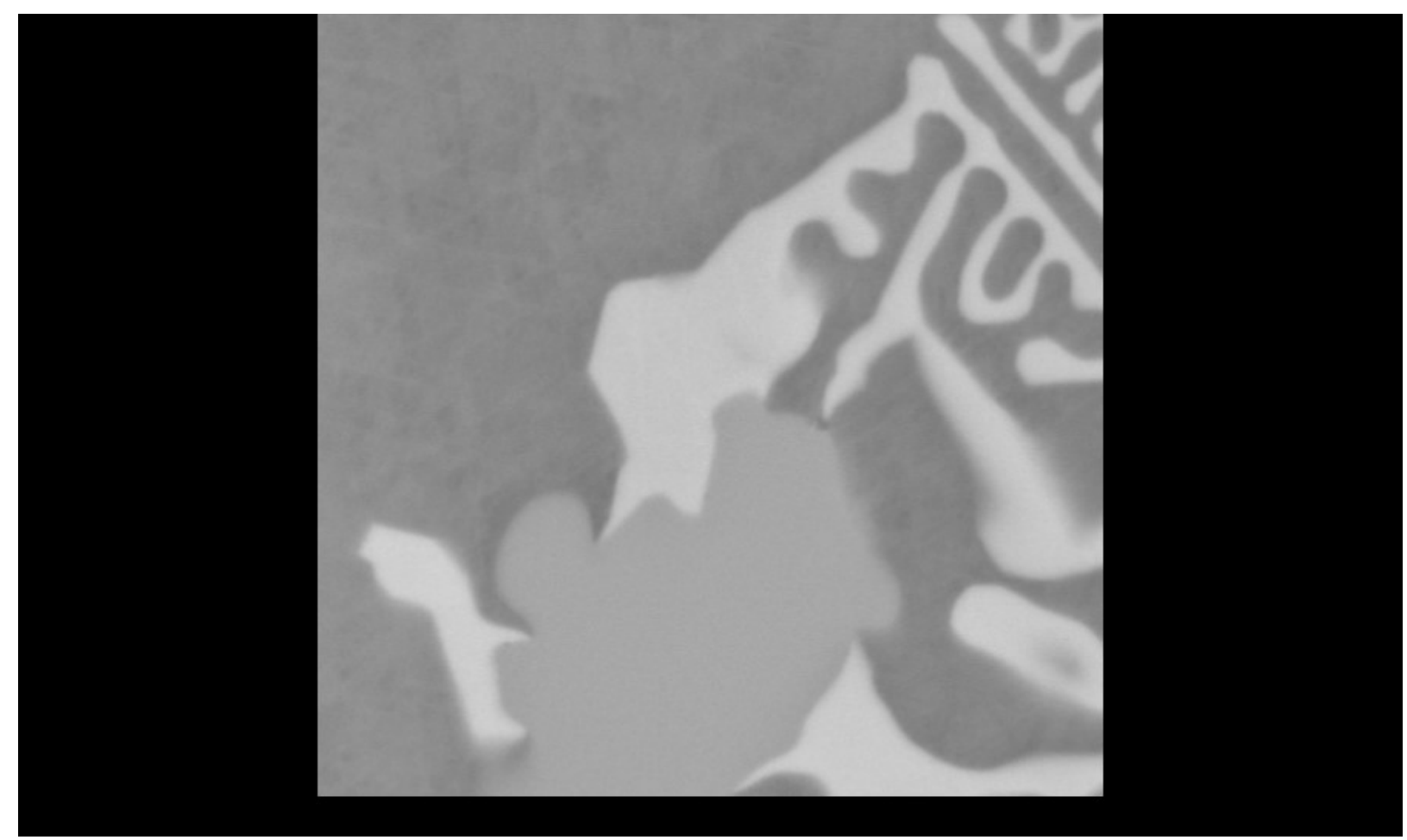

(a)
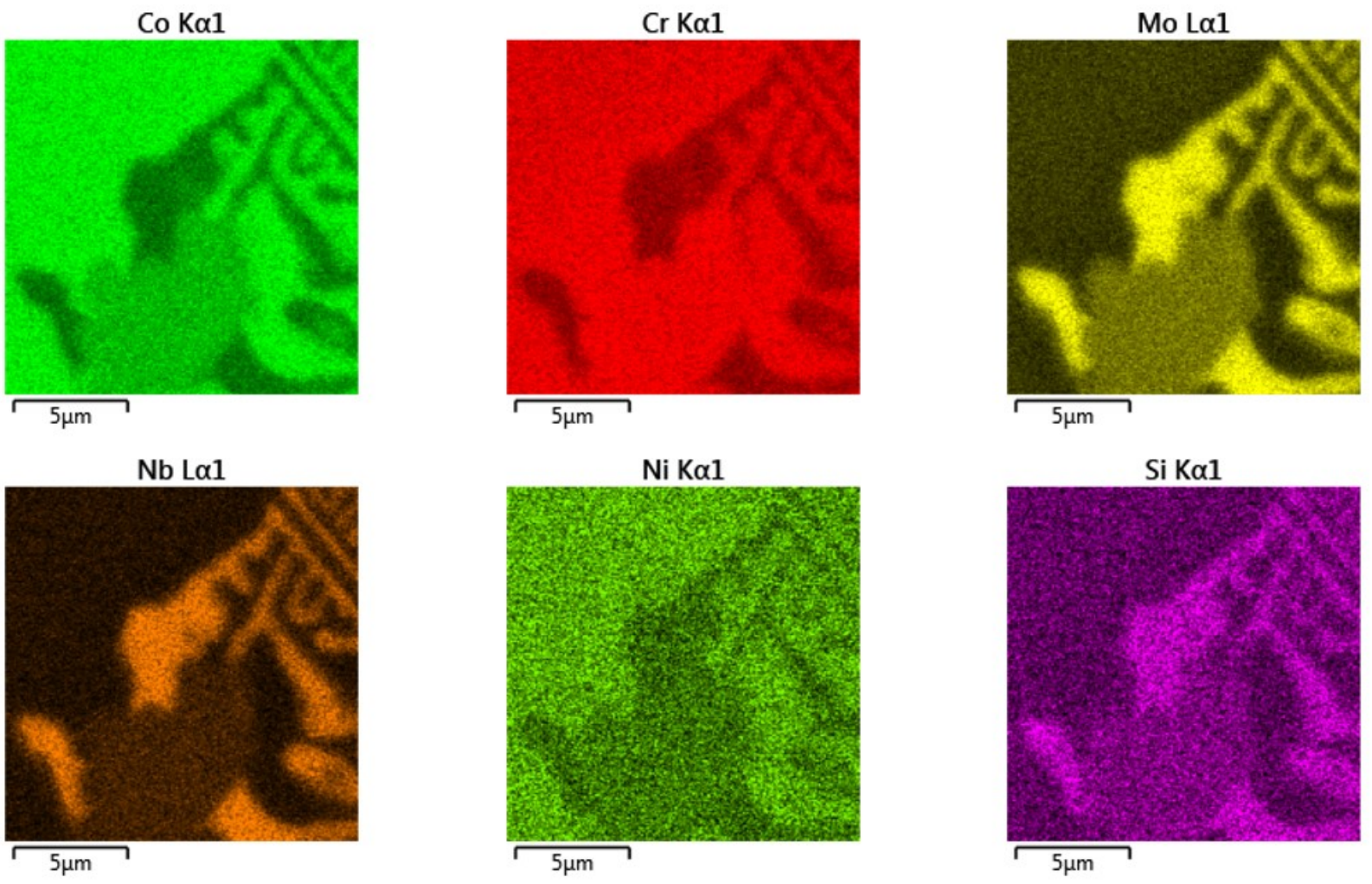

(b)

Figure 3-14: EDX map of as-cast Stellite 728 (a) EDX map image, (b) individual element maps. 


\subsection{XRD Analysis}

Identification of the phases proved to be very difficult with SEM/EDX, the multitude of elements detected revealed the complexity of the molybdenum Stellite structures. To complement the SEM/EDX analysis, XRD was used to generate a diffraction pattern for each of the three alloys. The diffraction pattern is essentially a fingerprint of the alloy, and this pattern can be interpreted using data cards from standards.

\subsubsection{XRD Data Collection}

The PANalytical XRD facility (Department of Earth Sciences, Carleton University), was utilized to identify sample phases in this study. The Gonio mode was adopted to scan sample surface from $20^{\circ}$ to $110^{\circ}$, and was operated at $40 \mathrm{kV}$ and $40 \mathrm{~mA}$ to generate monochromatic $\mathrm{Cu}$ $\mathrm{K} \alpha$ radiation. The XRD patterns were then analysed using analytical software, JADE 9 Plus, version 9.8.0, and coupled with the latest 2019 version of Powder Diffraction File (PDF) data base. The graphs were then imported to the software and smoothed one time. The data (PDF) cards showed varying degrees of reliability at source, so this added to the complexity of interpreting the diffraction pattern. There are also certain caveats for interpreting XRD curves which are:

i) The top three peaks are the most significant and must match.

ii) The intensity levels must match the same order of intensity as the XRD pattern being interpreted.

iii) The two-theta angle may be moved slightly left or right due to the alignment of the equipment, but the difference must be the same for all peaks on the PDF reference card. 
Taking these points into consideration, with only three or four elements selected, the number of potential PDF data cards can be in the hundreds. In addition, the combination of all the elements in Stellite alloys allows for many forms of carbides to be present. The standard nomenclature is to us $\mathrm{M}$ meaning any metal. However, some very complex structures were identified in the diffraction patterns.

The combined XRD patterns of the three alloys are shown in Figure 3-15, showing similarities in terms of the peak distribution and peak positions on the two-theta $(2 \theta)$ scale. However, Stellite 720 and Stellite 728 appear to be significantly more complex than Stellite 21.

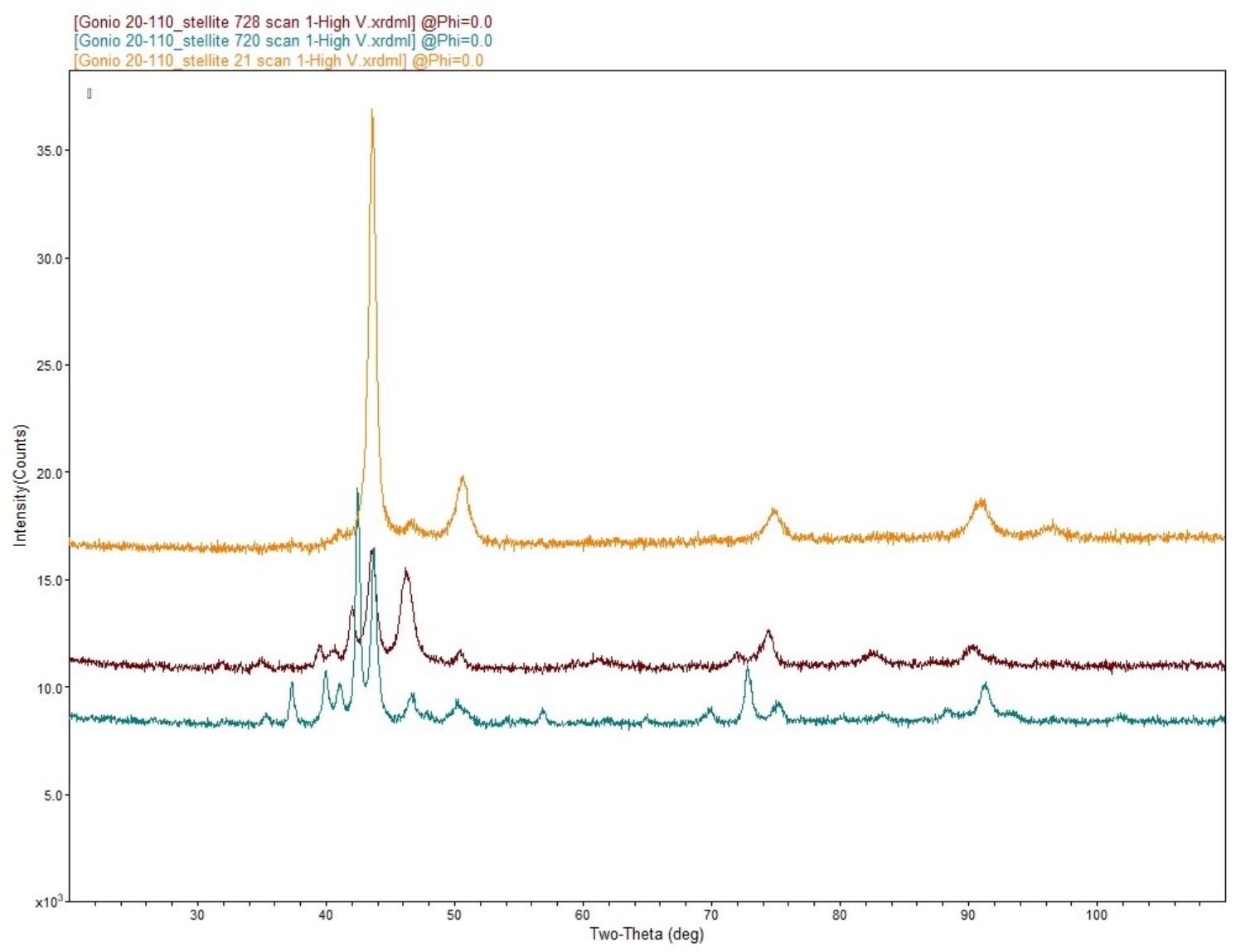

Figure 3-15: Combined XRD patterns for all three alloys 


\subsubsection{XRD Pattern of Stellite 21}

Stellite 21 is the least complex of the three alloys being studied in terms of the XRD pattern having the least amount of peaks. Evaluation is still difficult due to the complexity of the compounds being formed. However, the majority of the peaks resemble the expected $\mathrm{M}_{23} \mathrm{C}_{6}$ carbide, with varying degrees of $\mathrm{Cr}$ and Mo being present in the $\mathrm{M}$ portion of the compound. This is evident by the numerous amounts of applicable PDF cards some of which are listed in Figure 3-16. This confirms the major phase to be a eutectic mixture of various complex $\mathrm{M}_{23} \mathrm{C}_{6}$ carbides, with $\mathrm{M}_{\mathrm{x}} \mathrm{Ni}_{\mathrm{y}}$ intermetallic compounds where $\mathrm{M}$ can be $\mathrm{Cr}$, $\mathrm{Mo}$ or $\mathrm{Fe}$ of various ratios. The data cards show ratios such as $\mathrm{Cr}_{0.4} \mathrm{Ni}_{3.6}, \mathrm{Fe}_{3} \mathrm{Ni},(\mathrm{MoNi})_{0.4}$ and $\left(\mathrm{CrNi}_{9}\right)_{0.4}$. In one particular instance, two PDF cards show very similar compatibility in terms of intensity and $2 \theta$ placement to the XRD pattern. These two cards are shown in Figure 3-17 and Figure 3-18 and are different in elemental composition. One card shows a composition of $\left(\mathrm{MoNi}_{0}\right)_{0.4}$ and the other, $\mathrm{Cr}_{0.4 \mathrm{Ni}} \mathrm{Ni}_{\text {.6. }}$ This example follows true for the complexity in identifying the phases present in Stellite 720 and Stellite 728, and explains why in literature, the ' $\mathrm{M}$ ' nomenclature is used for the carbide identification.

To identify a phase in such complex alloy systems is a comprehensive work. It needs evidence from various perspectives, such as a precise chemical composition and accurate crystal structure, by using advanced analytical technologies, such as SEM, EDX, transmission electron microscopy (TEM), etc., all together. With the assistance of literature data, this thesis provides the phase identification to the author's best knowledge. The primary phases that constitute the microstructure and will contribute to the wear have been identified accordingly for the other alloys. The solid solution phases such as the elemental readings of $\mathrm{Co}, \mathrm{Cr}$ and Mo have been omitted to allow focus on the carbide and intermetallic phases. 


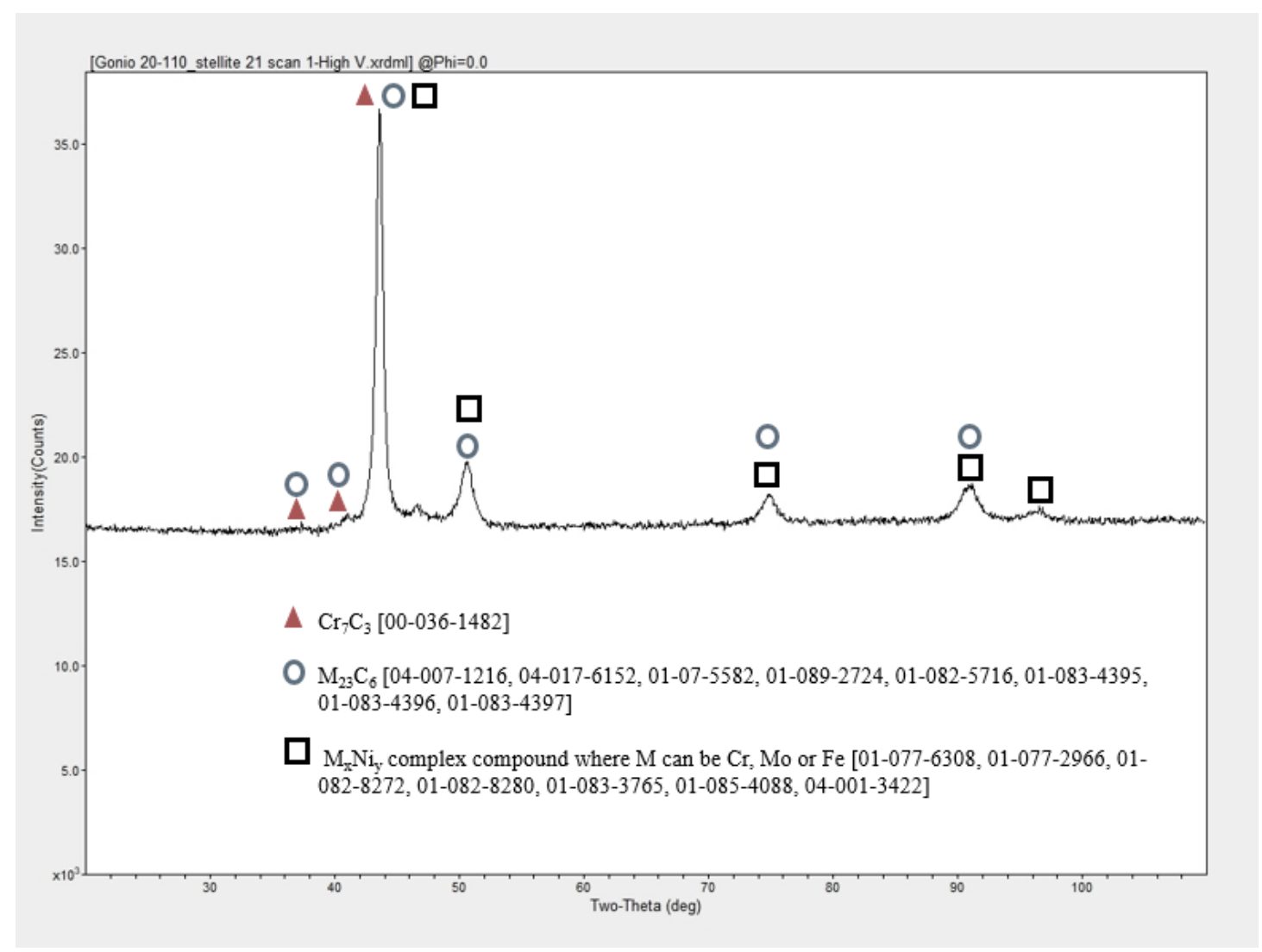

Figure 3-16: XRD pattern and peak identification for as-cast Stellite 21.

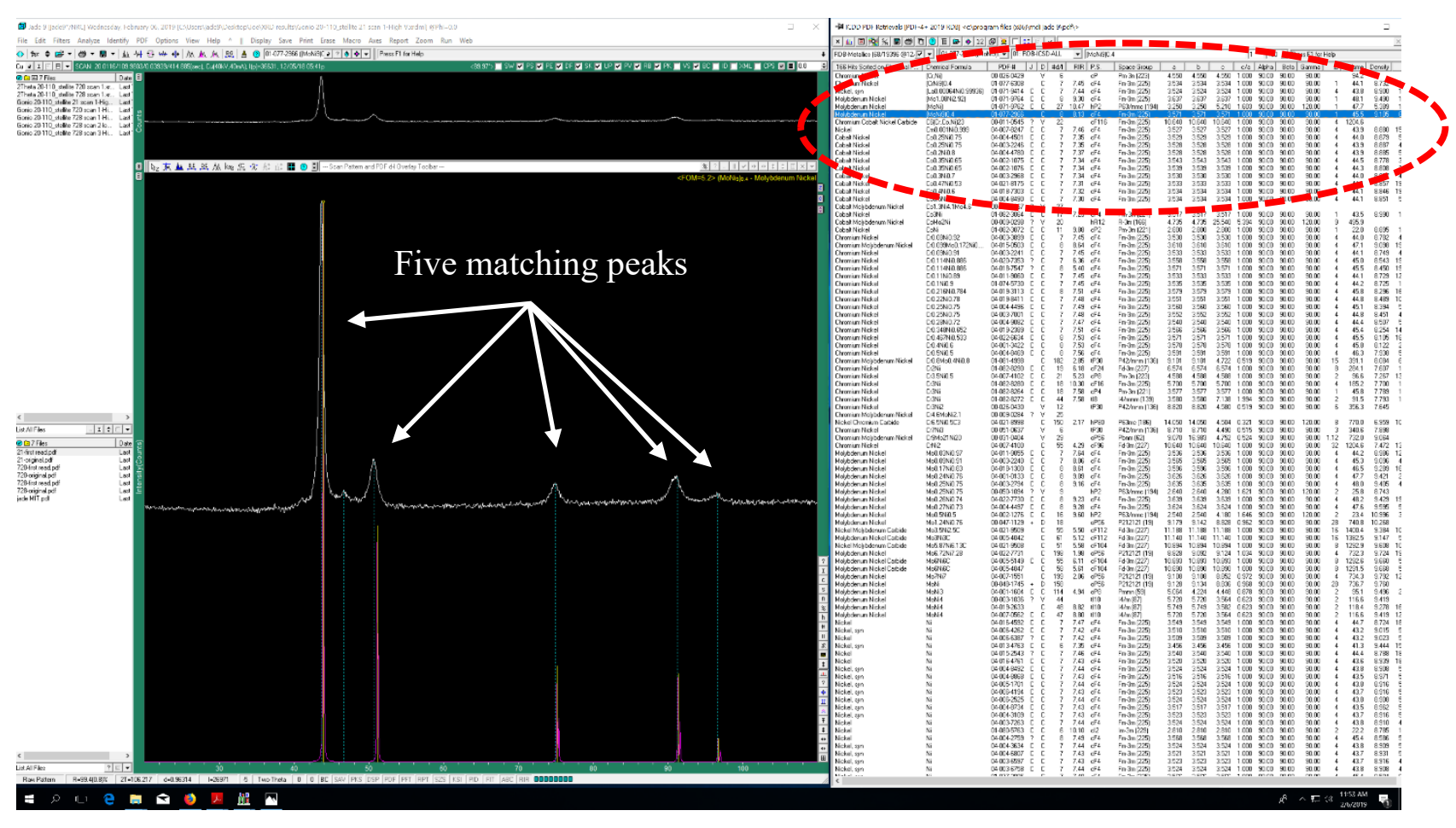

Figure 3-17: Matching PDF Card [01-077-2926] for (MoNi9)0.4. 


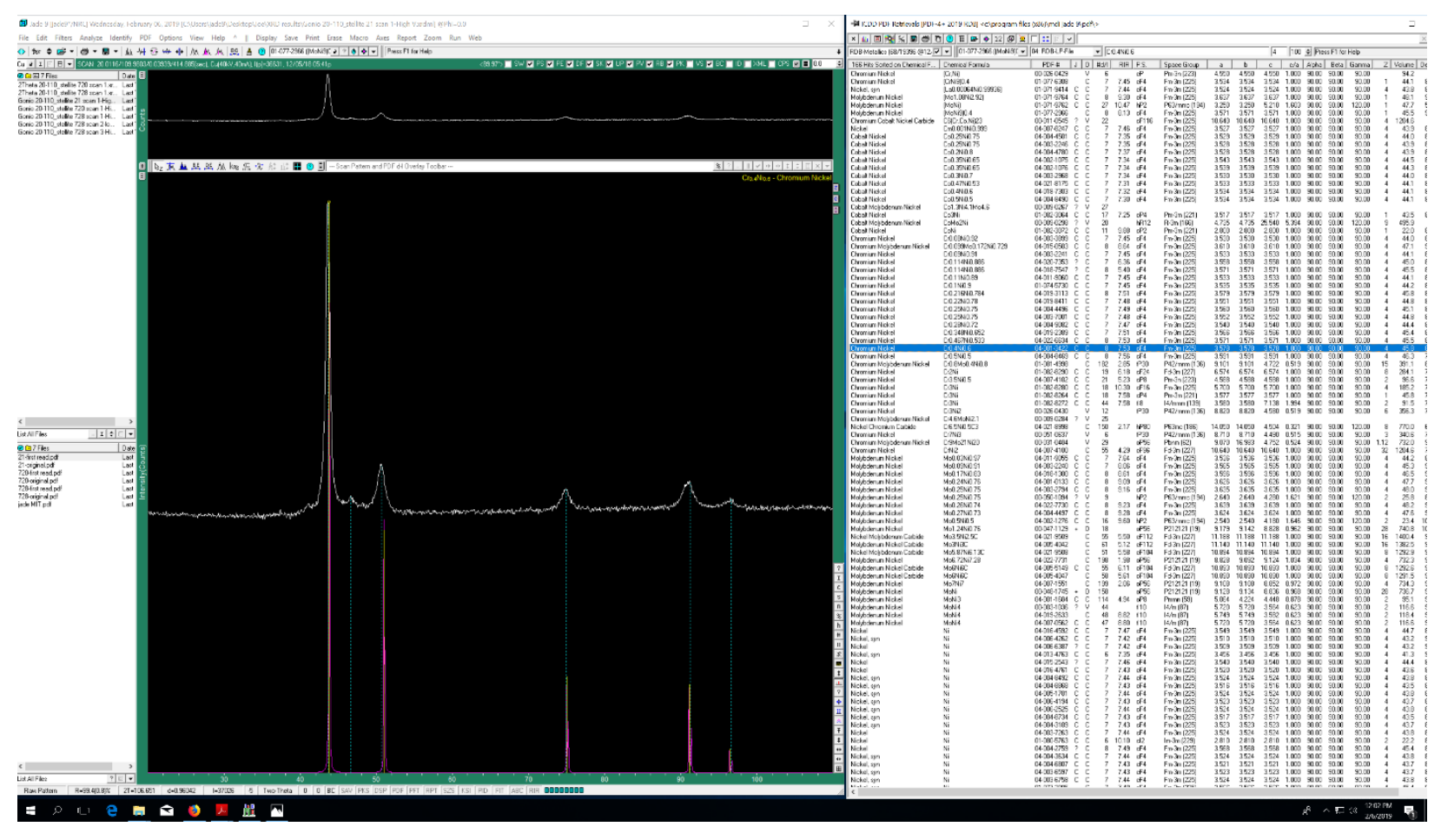

Figure 3-18: Matching PDF Card [01-077-2926] for $\mathrm{Cr}_{0.4} \mathrm{Ni}_{0.6}$.

\subsubsection{XRD Pattern of Stellite 720}

Stellite 720 is even more complex than Stellite 21 in terms of the number of potential phases present. The XRD pattern is shown in Figure 3-19, and the major peaks have been identified accordingly. The XRD analysis results show three types of carbides formed: $\mathrm{M}_{7} \mathrm{C}_{3}, \mathrm{M}_{6} \mathrm{C}$ and $\mathrm{M}_{23} \mathrm{C}_{6}$ in various compositions. An additional intermetallic phase of $\mathrm{Co}_{3} \mathrm{Mo}$ can also be identified. This data appears to match previous work [3], except for $\mathrm{M}_{23} \mathrm{C}_{6}$ being present in this work. This carbide has numerous compositions including $\mathrm{Cr}_{23} \mathrm{C}_{6}, \mathrm{Cr}_{21} \mathrm{Mo}_{2} \mathrm{C}_{6}, \mathrm{Cr}_{20} \mathrm{Mo}_{3} \mathrm{C}_{6}, \mathrm{Cr}_{16} \mathrm{Fe}_{5} \mathrm{Mo}_{2} \mathrm{C}_{6}$, $\left(\mathrm{Cr}_{0.77} \mathrm{Co}_{0.15} \mathrm{Mo}_{0.08}\right)_{23} \mathrm{C}_{6}$, and $\mathrm{Cr}_{7} \mathrm{MoCo}_{15} \mathrm{C}_{6}$. 


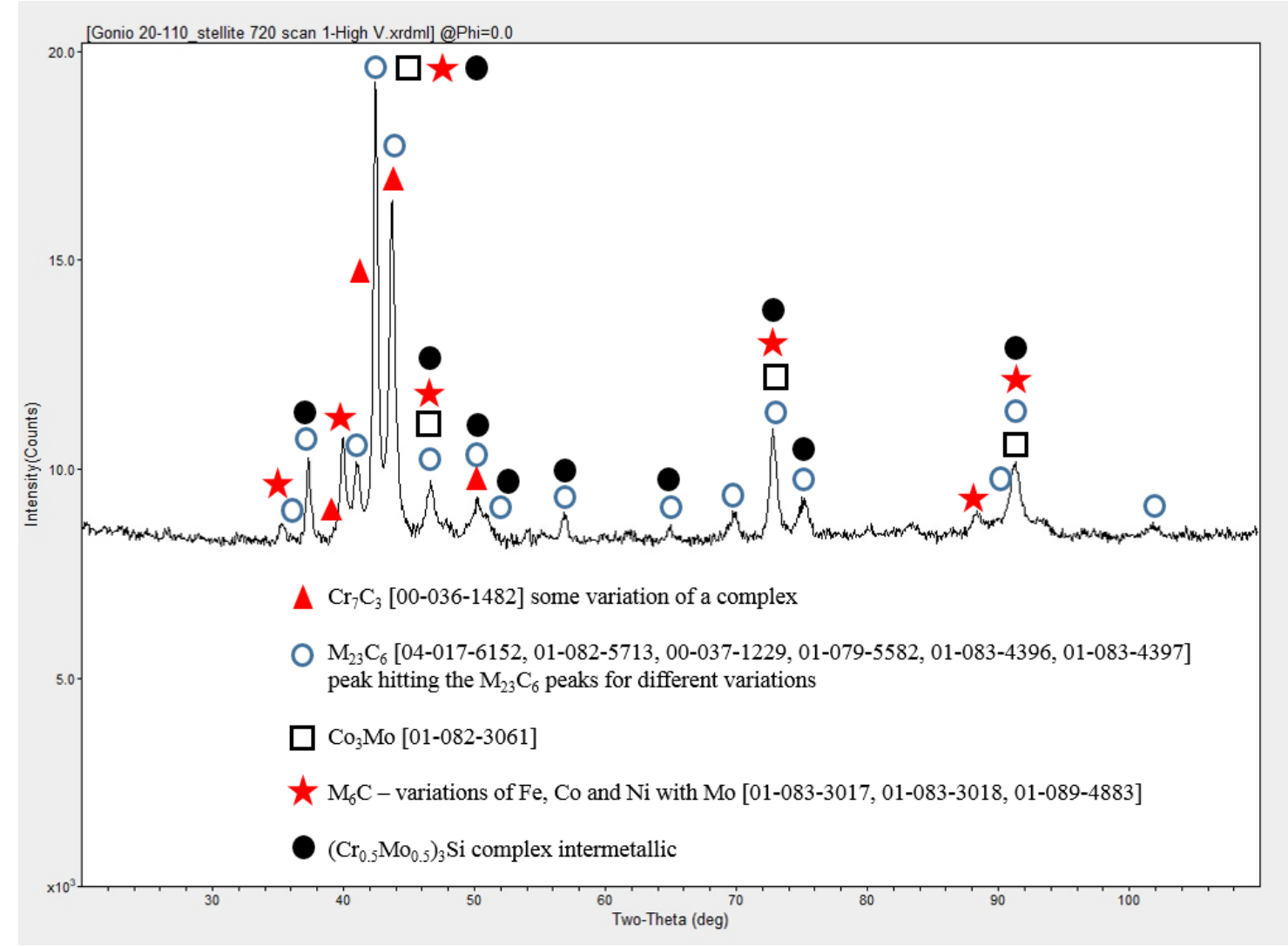

Figure 3-19: XRD pattern and peak identification for as-cast Stellite 720.

\subsubsection{XRD Pattern of Stellite 728}

Stellite 728 appears to be as complex as Stellite 720 in terms of the peak distribution and intensity. The XRD pattern is shown in Figure 3-20, and the major peaks have been identified accordingly. In addition to the three types of carbides: $\mathrm{M}_{23} \mathrm{C}_{6}, \mathrm{M}_{6} \mathrm{C}$, and $\mathrm{MC}_{4}$ in various compositions, and the intermetallic phase of $\mathrm{Co}_{3} \mathrm{Mo}$. The addition of niobium has increased the complexity of the interpretation. Additional complex carbide phases such as $\mathrm{NbCx}$, where $\mathrm{x}$ can represent 0.5 to 1 , and $\mathrm{Nb}_{6} \mathrm{C}_{5}, \mathrm{NbC}$, and an intermetallic $\mathrm{Nb}_{3} \mathrm{Mo} / \mathrm{Nb}$ phase. 


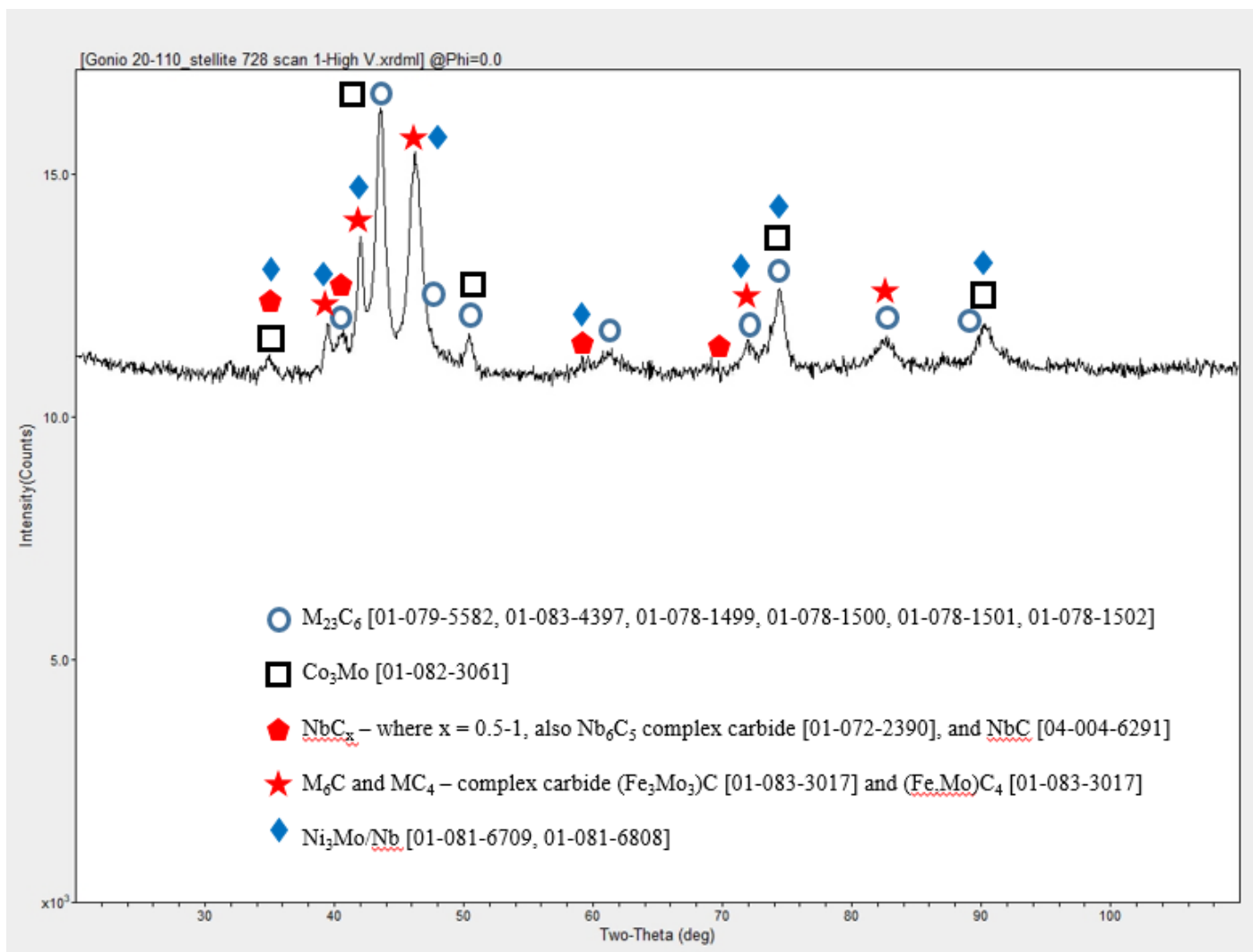

Figure 3-20: XRD pattern and peak identification for as-cast Stellite 728.

\subsection{Carbide Volume Fraction Measurement}

In general, the hardness of a material is proportional to the carbide volume fraction of the material. In the present work a measurement was conducted on the polished samples to ascertain the volume fraction for the three alloys being tested, which was performed with the ATLAS image processing software coupled with the TESCAN SEM. The volume fraction was calculated by converting the greyscale image into a binary image (thresholding). The level of thresholding depended on the contrast in the image, and with Stellite alloys, the BSEs can assist with the imaging process by highlighting the different phases (grey tones and white).

Figure 3-21, to Figure 3-23 show the SEM images and the resulting threshold (binary) coloured images for Stellite 21, Stellite 720 and Stellite 728 respectively. A calculation using the 
software was made based on the percentage area covered using the threshold colouring. Based on the information found in the SEM/EDX and XRD evaluation, the phases are not only carbide-type, but intermetallic phases are also present. Therefore the hard phases including carbides and intermetallics can be calculated in the volume fraction measurement.

The volume fraction of hard phases in Stellite 21 and Stellite 728 were relatively straightforward to calculate using the software. In the case of Stellite 720 , the microstructure is packed full of distinct phases. However, the thresholding did have limitations to the calculations, and the very fine medium grey and light grey phases identified in Figure 3-9, were difficult to delineate.

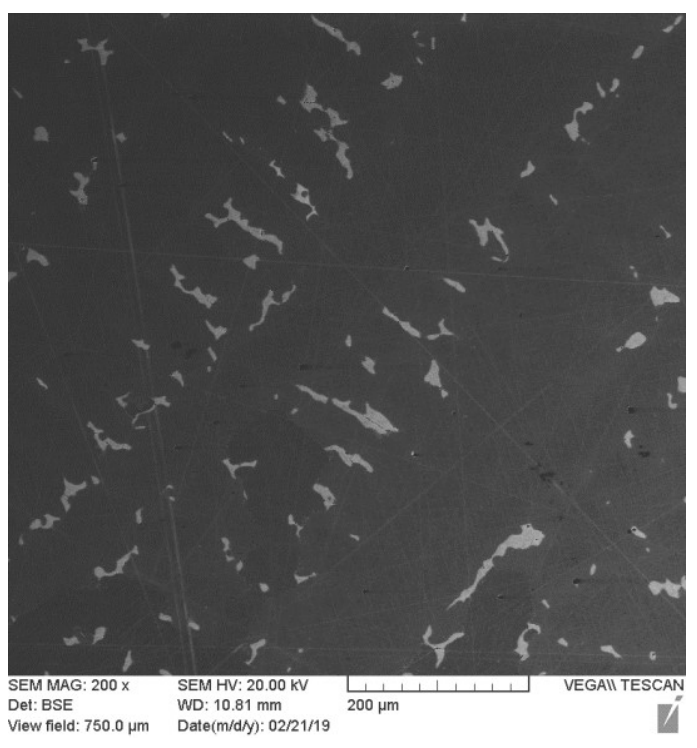

(a)

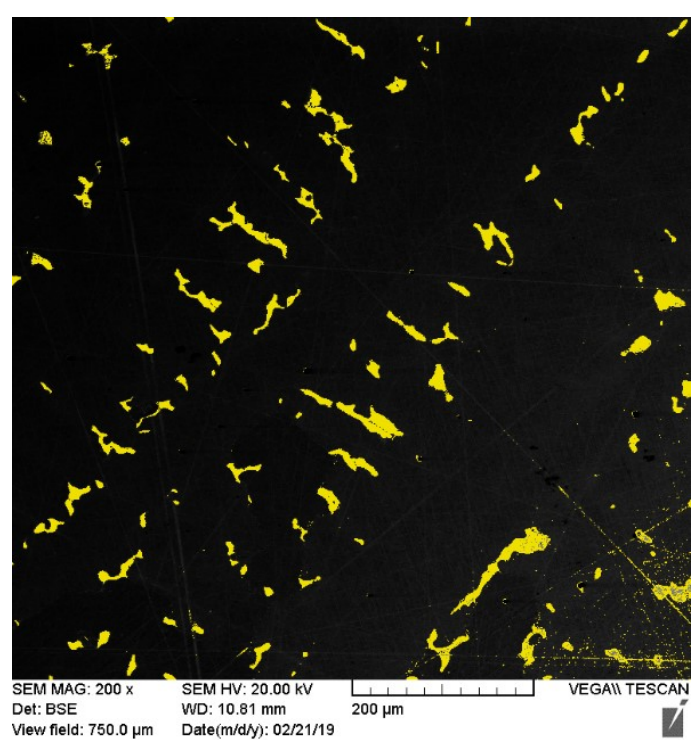

(b)

Figure 3-21: Carbide volume fraction images of as-cast Stellite 21 (a) SEM image, (b) thresholding image analysis 


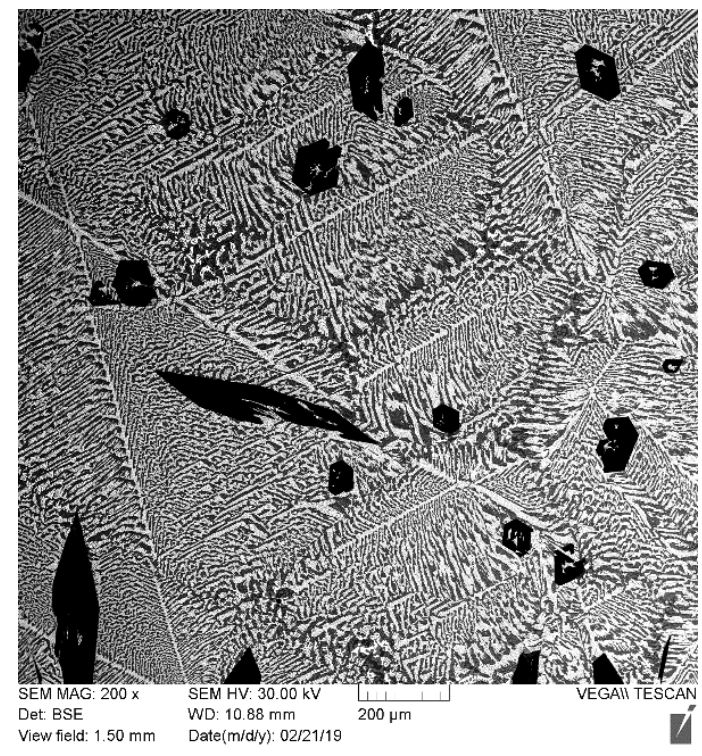

(a)

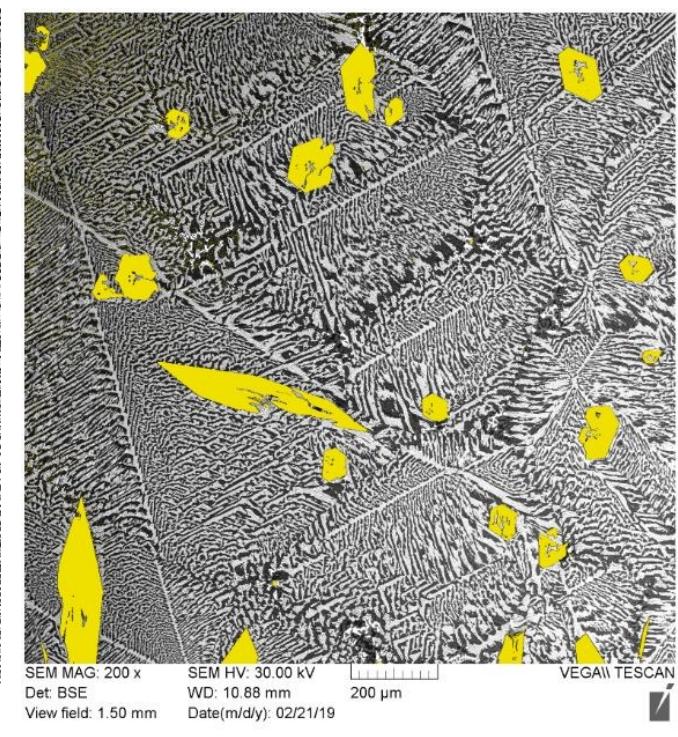

(b)

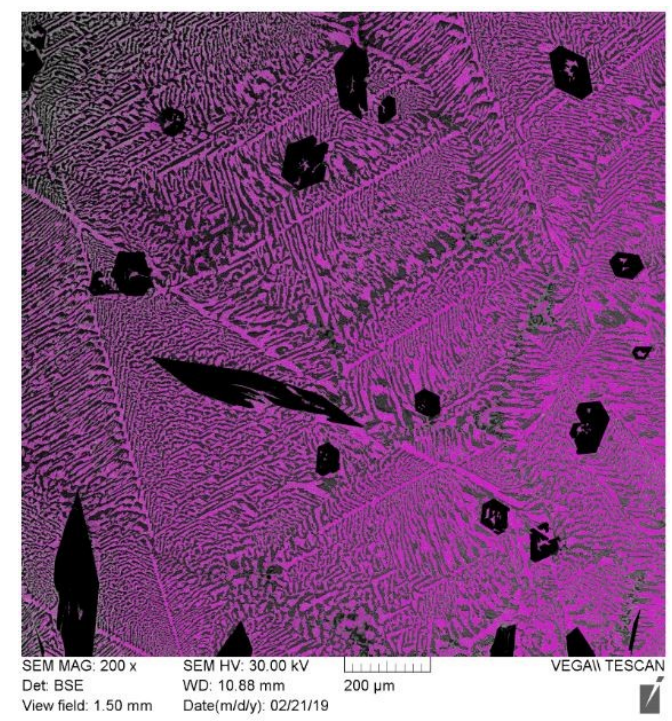

(c)

Figure 3-22: Carbide volume fraction of as-cast Stellite 720 (a) SEM image, (b) thresholding image analysis of the large blocky carbide, (c) thresholding image analysis of the dendritic phase 


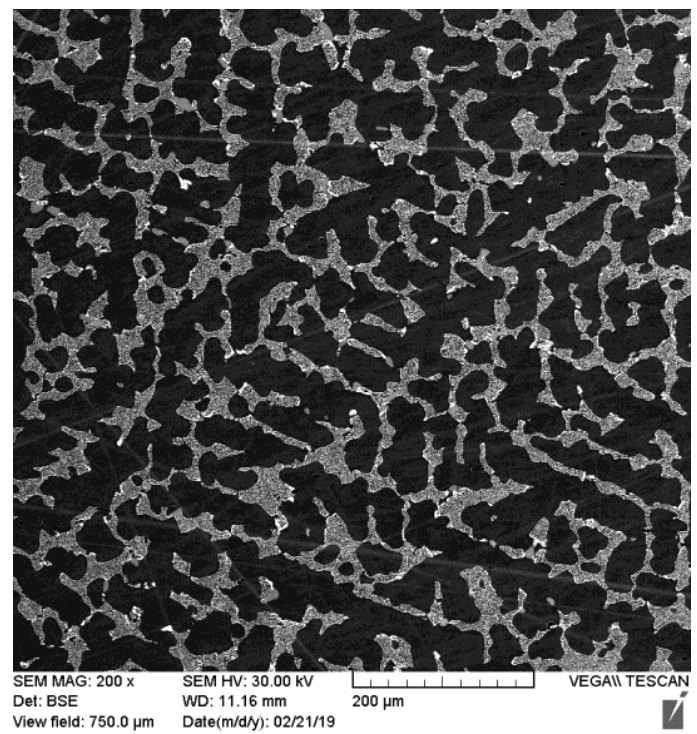

(a)

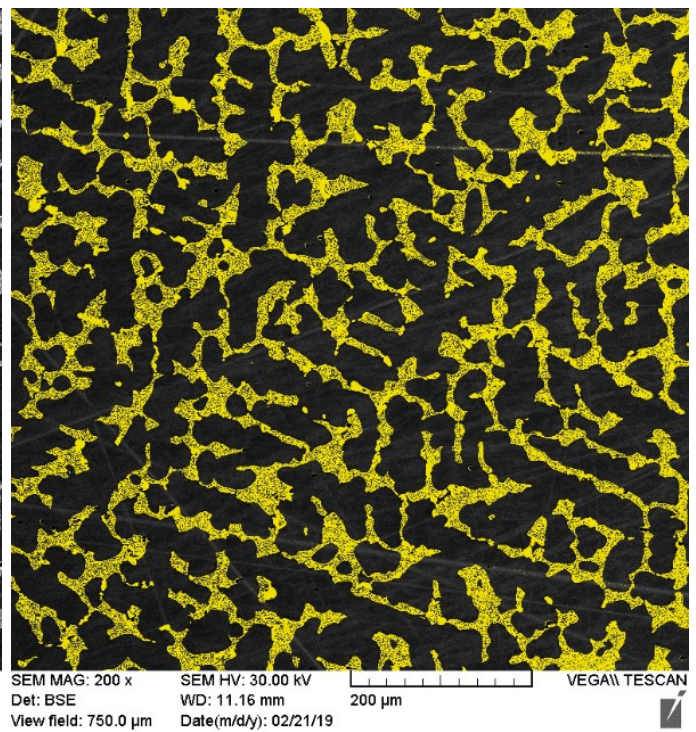

(b)

Figure 3-23: Carbide volume fraction images of as-cast Stellite 728 (a) SEM image, (b) thresholding image analysis highlighting hard phases.

Three readings were taken and averaged to calculate the volume fraction of hard phases in each of the three Stellite alloys.

Table 3-2: Volume fractions of carbides and intermetallics from the selected Stellite alloys

\begin{tabular}{|c|c|c|}
\hline Alloy & $\begin{array}{c}\text { Carbide and } \\
\text { intermetallic }\end{array}$ & Solid solution \\
\hline Stellite 21 & $4.6 \%$ & $95.4 \%$ \\
\hline Stellite 720 & $\begin{array}{c}\text { Blocky phase 7.5\%, } \\
\text { Dendritic 61.8\% }\end{array}$ & $30.8 \%$ \\
\hline Stellite 728 & $21.0 \%$ & $79.0 \%$ \\
\hline
\end{tabular}




\subsection{Summary of SEM/EDX and XRD Analysis}

Stellite 21 is solution-strengthened rather than carbide-strengthened so that it has a microstructure with a very small volume fraction of carbides (4.6\%) compared to Stellite 720 $(>60 \%)$ and Stellite 728 (21\%). Two types of carbides are present in its microstructure, $\mathrm{Cr}_{7} \mathrm{C}_{3}$ and $\mathrm{M}_{23} \mathrm{C}_{6}$, as well as a complex compound of $\mathrm{M}_{\mathrm{x}} \mathrm{Ni}_{\mathrm{y}}$ type.

Stellite 720 has a very compact microstructure with four distinct phases of varying degrees of complexity. The large hexagonal phase is reported to be a Cr-rich carbide of $\mathrm{Cr}_{7} \mathrm{C}_{3}$ type [3]. However, the PDF cards only showed some alignment with this phase in the present research. The solid solution intermingles with the other two dendritic phases of $\mathrm{M}_{23} \mathrm{C}_{6}$ complex carbides, $\mathrm{M}_{6} \mathrm{C}$ carbides, with intermetallics also being present.

Stellite 728 is the most complex of these three alloys having a wide variety of complex carbide and intermetallic phases. In general, this microstructure contains a network of two fine hard carbides and intermetallics within a solid solution of Co-Cr-Mo. The fine carbide network contains complex carbides of $\mathrm{M}_{23} \mathrm{C}_{6}, \mathrm{MC}_{4}, \mathrm{Nb}$ complex carbides, $\mathrm{Ni}_{3} \mathrm{Mo} / \mathrm{Nb}$, and $\mathrm{Co}_{3} \mathrm{Mo}$. 


\section{Wear Testing and Modeling}

\subsection{Pin-on-Disc Test}

As with many applications, special test apparatus would be needed to study the material behaviour associated with particular failure mode. Sliding wear test methods have been developed to study the wear of materials. The American Society for Testing of Materials (ASTM) has specified at least three types of sliding wear tests: ASTM G99 Standard Test Method for Wear Testing with a Pin-on-Disk Apparatus, ASTM G77 Standard Test Method for Ranking of Materials to Sliding Wear Using a Block-on-Ring Wear Test, and ASTM G98 Standard Test Method for Galling Resistance of Materials. These wear tests can be conducted in either lubricated or unlubricated (dry) condition.

A wear test usually employs either of the two methods to record the material loss: a) by removing the specimen at prescribed intervals and taking measurements, or b) by the use of an electrical or mechanical transducer which constantly monitors the dimensional changes in the system. From these measurements, the wear rate can be calculated.

During wear testing, the apparatus should record the friction force by measuring the tangential force (specimen) or torque (rotating counterface). Continuous monitoring can allow the analysis of oxide layer breakdown and the 'running-in' (transient) period before the steady-state wear is established. Eight types of friction running-in curves are shown in Figure 4-1 [18]. Each curve is not indicative of a single process or a unique combination of processes, so each curve must be analyzed according to the given tribo-system. The curve shown in Figure 4-1a, for example, is characteristic of a dry, unlubricated metal couple where a surface layer of some description, e.g. an oxide layer, has been quickly worn away to cause a significant rise in friction i.e. adhesion [18]. After a period of time, a steady-state (surface equilibrium) is established where 
the friction force is constant and the adhesion has stabilized. The behavior shown in Figure 4-1 (e) shows a marked transition from a relatively stable friction force to a higher friction force, which could be considered a break-in effect, but a longer duration would be required to establish a more conclusive result.

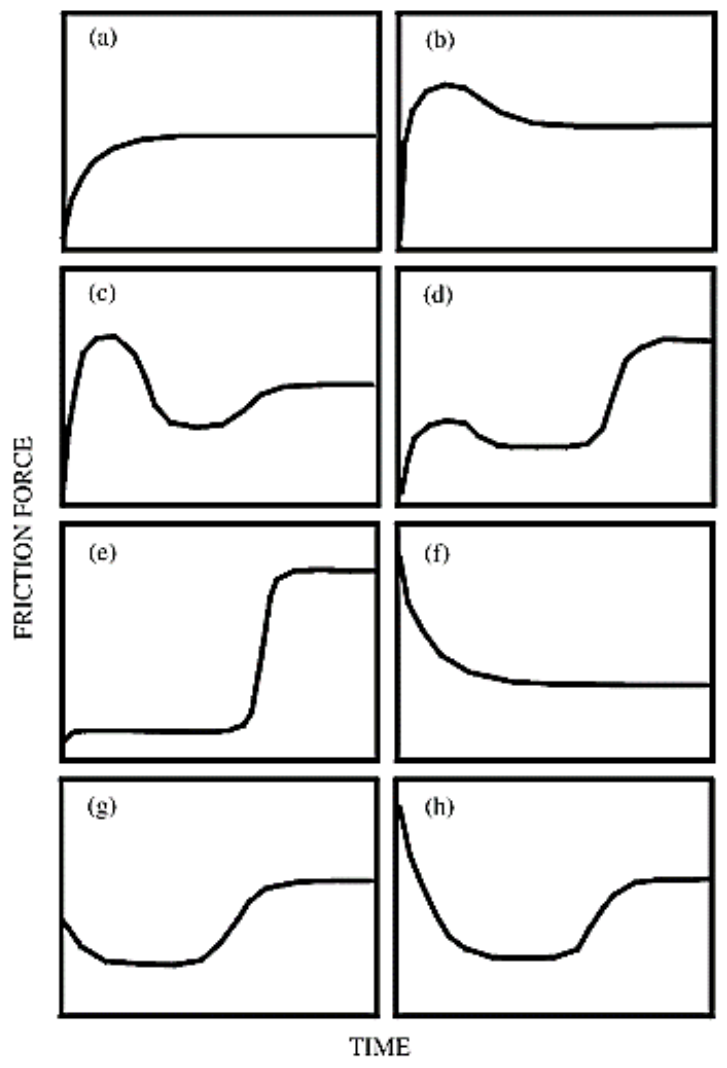

Figure 4-1: Types of break-in curve shapes, friction force versus time [18].

\subsubsection{Testing Facilities}

The wear testing in the present study was conducted using a NEO-PLUS, NEO-TRIBO Model MPW110 Pin-on-Disc Tribometer, in accordance with ASTM G 99 - 17 Standard Test Method for Wear Testing with a Pin-on-Disc Apparatus. The particular method employed from this standard incorporates a ball mounted in a holder (pin section) which sits directly on top of a disc (specimen). In the apparatus used for the present research shown in Figure 4-2 the ball holder 
is the rotating part, and the load is applied through the counter weights on the disc. During the test the pin (ball) was pressed under a normal force on the specimen without lubrication and the ball was spinning at a constant rotational speed. As the result of friction/wear, a wear track or pit was generated on the specimen surface, as shown in Figure 4-3. Sensors are loaded onto the apparatus to provide feedback of resistance experienced by the disc rotating mechanism, this in turn will calculate the friction coefficient based on the inputs into the control software.

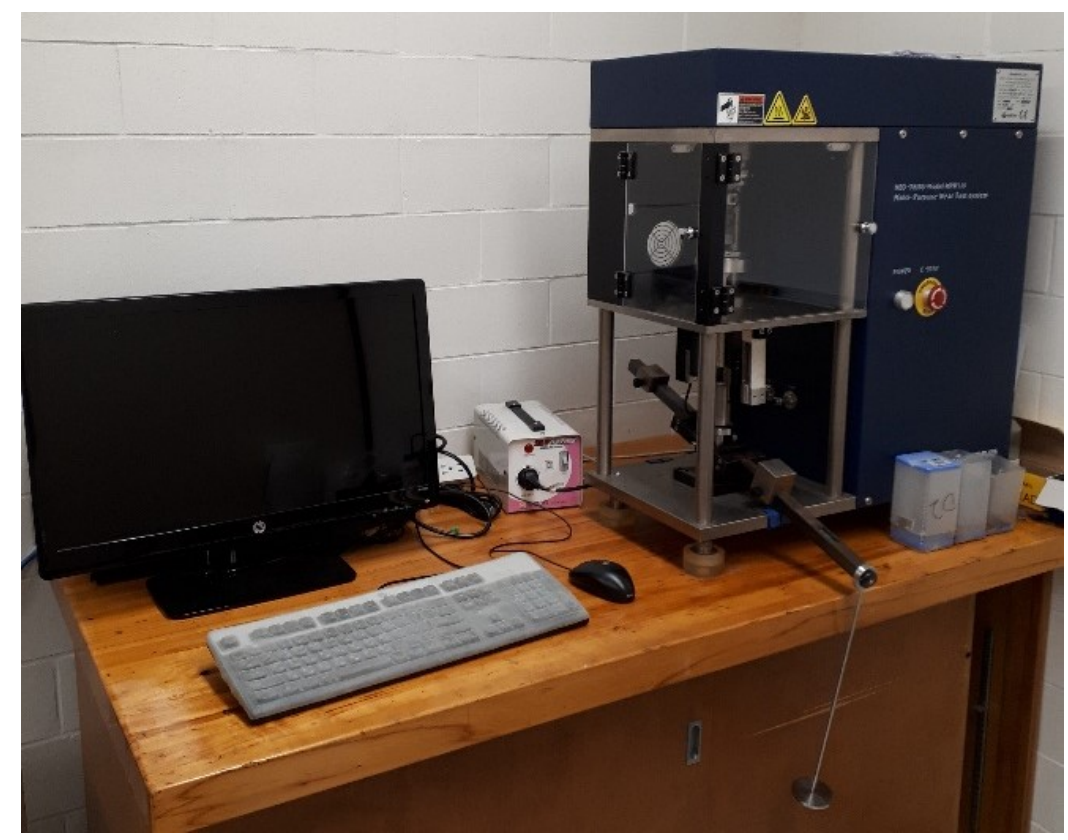

Figure 4-2: Pin-on-disc apparatus and computer control. 


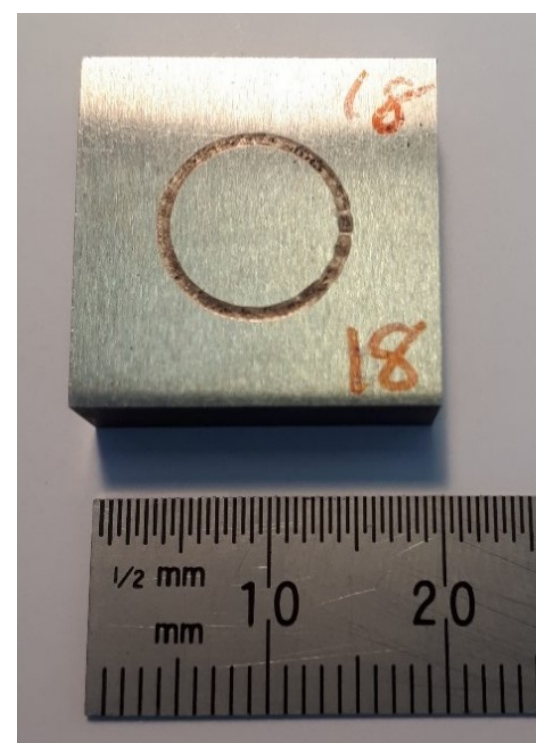

(a)

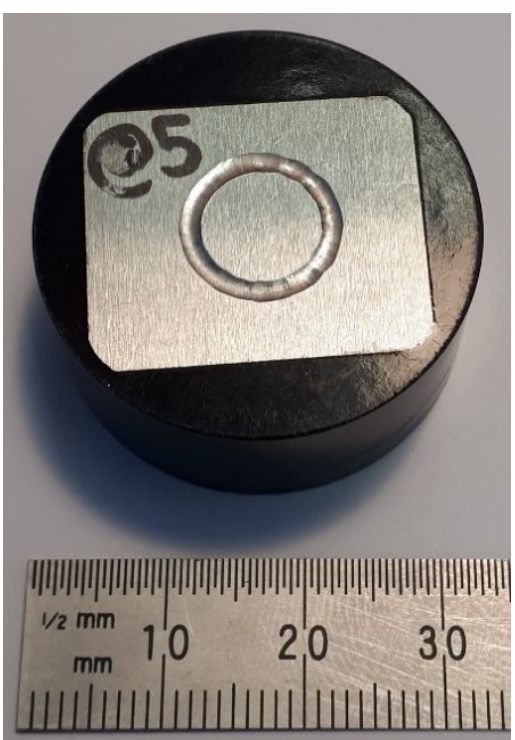

(b)

Figure 4-3: Two types of specimens used for wear testing (a) stand alone specimen (Stellite 720), (b) mounted specimen (Stellite 21, Stellite 728).

The pin is a $5 \mathrm{~mm}$ diameter ball, manufactured from tungsten carbide (WC 93.5 - 94.5 wt.\%) and cobalt (5.5 - 6.5 wt.\%). The hardness of the ball material is expected to be $\sim 1500 \mathrm{HV}$. The disc specimens are approximately $20 \times 20 \times 6 \mathrm{~mm}$ (Figure 4-3). In the case of Stellite 21 and Stellite 728 , these samples are thinner so that they were mounted in thermo-setting resin to increase the height for clearance in the disc fixture (Figure 4-3). All specimens were ground with a surface roughness of $<0.8 \mu \mathrm{m}$ arithmetic average or less as per ASTM G99. A typical set-up for the pinon-disc tester is shown in Figure 4-4. 


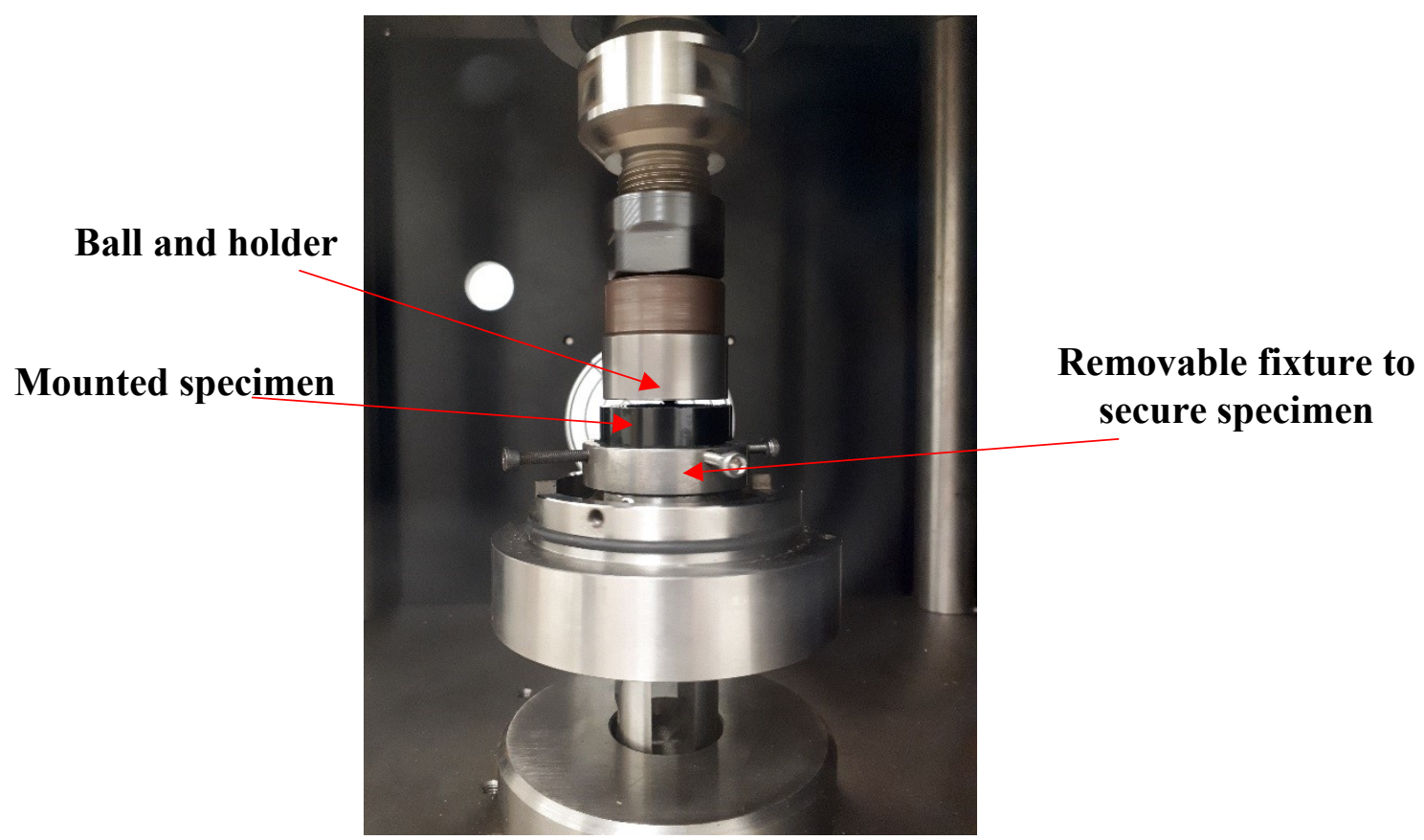

(a)

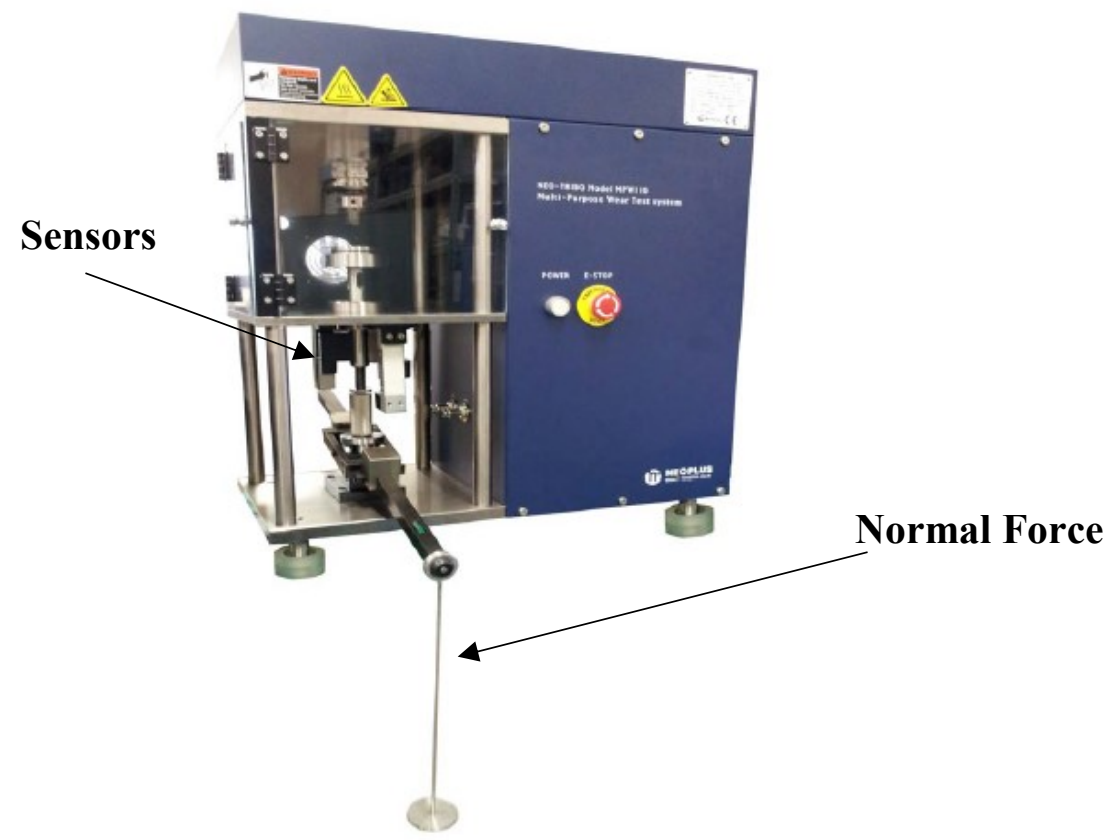

(b)

Figure 4-4: Pin-on-disc wear testing set-up (a) specimen in fixture, (b) overall structure of pin-on-disc tester. 


\subsubsection{Test Parameters and Data Collection}

To investigate the tribological behaviour of the three Stellite alloys, a test matrix was designed to accommodate three variables, (1) change in time/duration of the test, (2) normal load applied to the system, and (3) rotation speed of the ball. A summary of the variables and set parameters required for testing is given in Table 4-1.

Table 4-1: A summary of wear test parameters

\begin{tabular}{|l|l|}
\hline \multicolumn{1}{|c|}{ Parameter } & \multicolumn{1}{c|}{ Value(s) } \\
\hline Test Duration/Time & $1 \mathrm{hr}$ up to $60 \mathrm{hrs}$ \\
\hline Data Collection Intervals & $\begin{array}{l}\text { 100 data points were taken automatically for 2 seconds } \\
\text { at the beginning of the test to measure the coefficient } \\
\text { of static friction. Thereafter, a user setting of } 0.5 \mathrm{~Hz} \\
\text { (every two seconds) was used. }\end{array}$ \\
\hline Load (normal force) & $15 \mathrm{~N}$ and $25 \mathrm{~N}$ \\
\hline Rotation Speed & $50 \mathrm{rpm}$ and $60 \mathrm{rpm}$ \\
\hline Wear Track Radius & $5 \mathrm{~mm}$ \\
\hline Test Temperature & Room temperature $\left(\sim 20^{\circ}\right)$ \\
\hline
\end{tabular}

Wear tests were conducted in two modes: (a) continuous, and (b) interrupted (interval measurements) tests. The continuous tests involved the specimens being run to the designated duration without interruption. On the contrary, the interrupted tests were stopped several times for data collection. For example, if one test was scheduled to run 40 hours, it would be interrupted at the $1,3,5,7,9$ hour time intervals etc. At the stops, wear loss data points were measured and the specimen was returned to the test machine. The purpose of doing this was to ascertain early changes in the wear process and to gather more data in terms of wear loss over a given distance.

A drawback for the interrupted test was that any wear debris on the worn surface would be lost during the wear loss measurement process by cleaning and transporting the specimen to the measurement equipment. The removal of the wear debris may change the dynamics of the wear 
system since debris can act as abrasive particles between the contacts as previously discussed in Chapter 2. Continuous tests were performed at $50 \mathrm{rpm}$ under the normal load of $15 \mathrm{~N}$ and $25 \mathrm{~N}$, respectively. Interrupted tests were performed at both $50 \mathrm{rpm}$ and $60 \mathrm{rpm}$, again under the normal load of $15 \mathrm{~N}$ and $25 \mathrm{~N}$. A comparison between the continuous and interrupted tests was of interest, from service profile point of view, which would be part of the analysis. Preliminary data gathered from the continuous tests showed that $15 \mathrm{~N}$ load did not have a significant impact on the wear loss for any of the three alloys. Therefore, the interrupted tests were chosen to be performed using the load of $25 \mathrm{~N}$ only (Table 4-2). To include modeling as a part of the present research, an increased rotation speed was chosen to validate the findings. An example of wear data generated by the tribometer is presented in Figure 4-5. The sensors can measure the wear loss in terms of the depth changes, however, a direct depth measurement was considered to be more accurate and was used to calculate the volume loss.

Table 4-2: Test matrix showing three variables and method of data collection

\begin{tabular}{|c|c|c|c|c|c|}
\hline \multicolumn{2}{|c|}{$50 \mathrm{rpm}$ continuous test } & \multicolumn{2}{|c|}{$50 \mathrm{rpm}$ interrupted test } & \multicolumn{2}{|c|}{$60 \mathrm{rpm}$ interrupted test } \\
\hline $\begin{array}{l}\text { Force } \\
(\mathbf{N})\end{array}$ & $\begin{array}{c}\text { Duration/Time } \\
\text { (hrs) }\end{array}$ & $\begin{array}{l}\text { Force } \\
(\mathbf{N})\end{array}$ & $\begin{array}{c}\text { Duration/Time } \\
\text { (hrs) }\end{array}$ & $\begin{array}{l}\text { Force } \\
(\mathbf{N})\end{array}$ & $\begin{array}{c}\text { Duration/Time } \\
\text { (hrs) }\end{array}$ \\
\hline 15 & 10 & \multirow{6}{*}{25} & \multirow{6}{*}{$\begin{array}{c}\text { Time intervals } \\
\text { approximately at } 1 \text {, } \\
3,5,7,9,10,15, \\
20,30 \text { and } 40\end{array}$} & \multirow{6}{*}{25} & \multirow{6}{*}{$\begin{array}{l}\text { Various time } \\
\text { intervals from } 1 \\
\text { up to and } \\
\text { including } 60\end{array}$} \\
\hline 15 & 20 & & & & \\
\hline 15 & 40 & & & & \\
\hline 25 & 10 & & & & \\
\hline 25 & 20 & & & & \\
\hline 25 & 40 & & & & \\
\hline
\end{tabular}




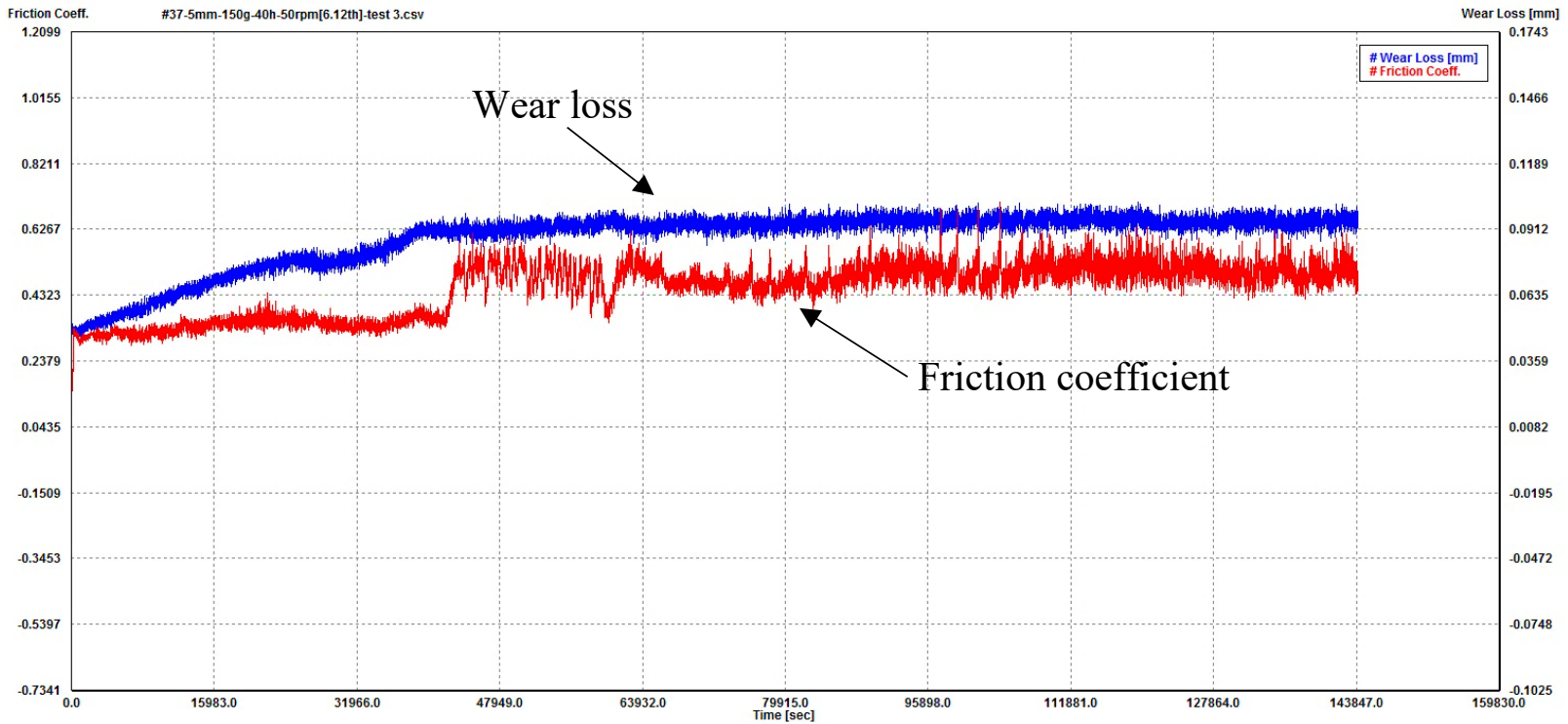

Figure 4-5: Continuous raw wear data of Stellite 21 under $15 \mathrm{~N}$ at $50 \mathrm{rpm}$.

After each continuous and/or interrupted interval, the wear loss was calculated via a 2D profilometer D150 Surface Profile Measuring System, shown in Figure 4-6, which computes the cross-sectional area of the wear track directly. Examples of the wear tracks on the tested alloys are shown in Figure 4-7 to Figure 4-11. In presenting the continuous testing, single data points were selected to represent the trend in the results. Some repeat tests were carried out but not on every data point. 


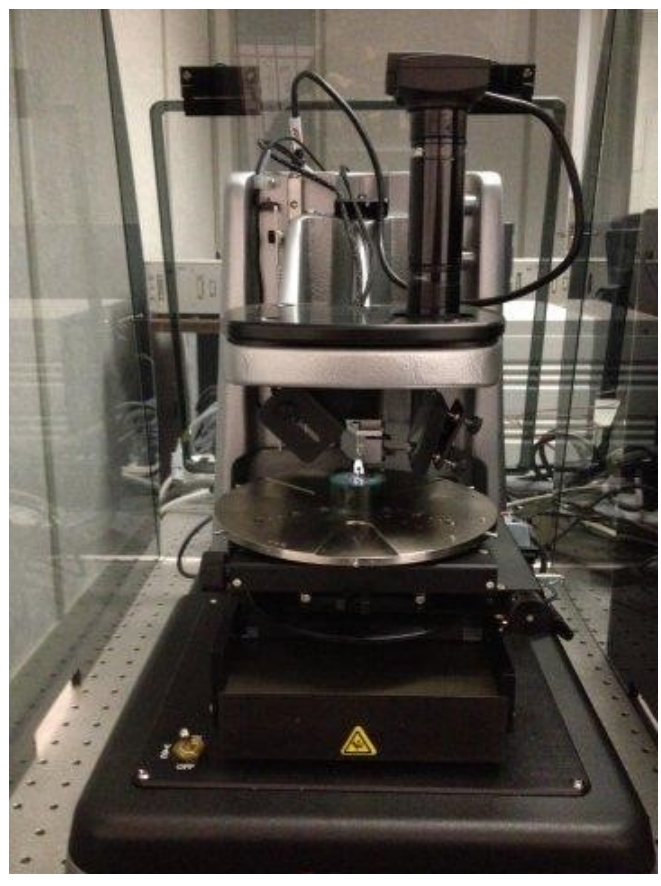

Figure 4-6: D150 Surface Profile Measuring System.

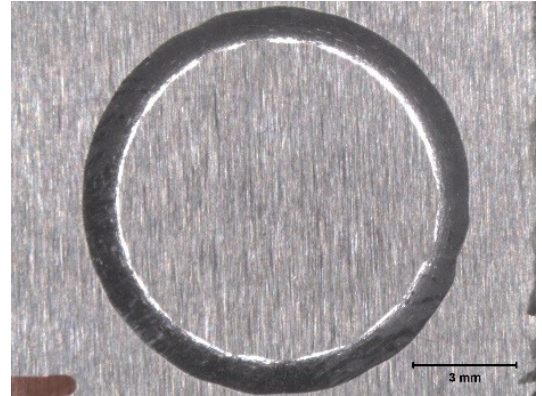

(a)

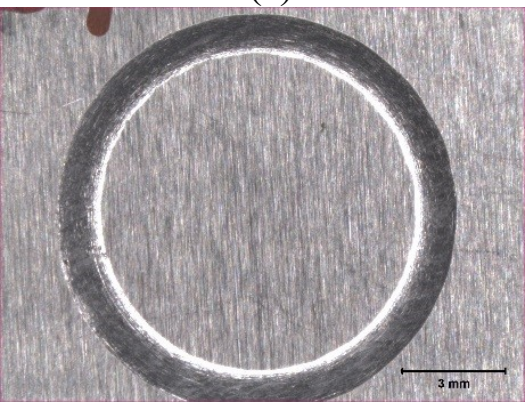

(d)

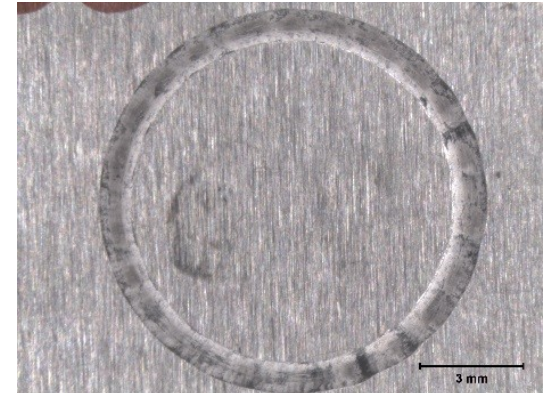

(b)

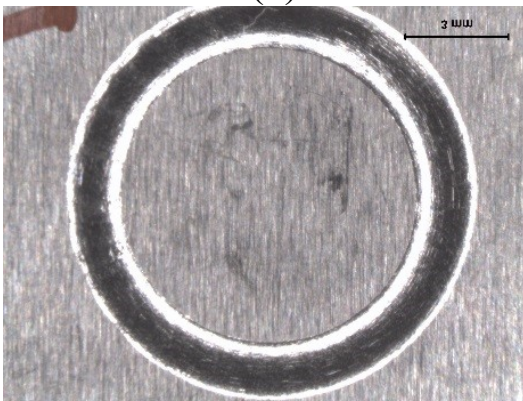

(e)

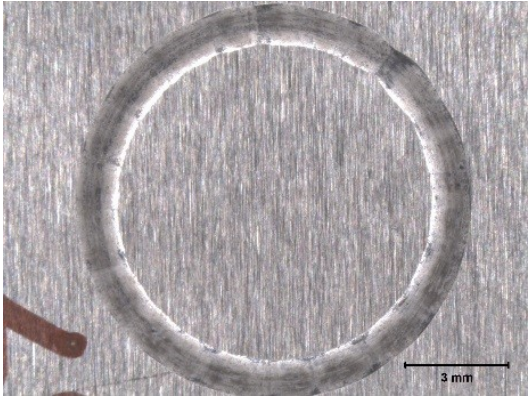

(c)

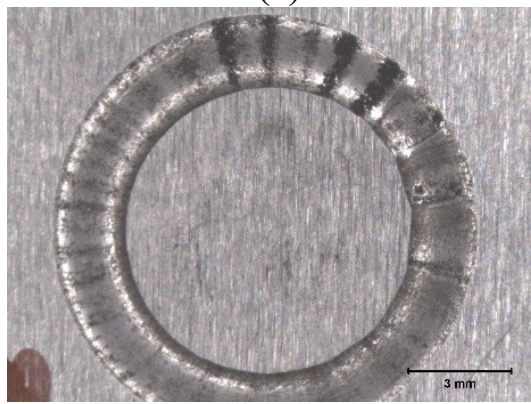

(f)

Figure 4-7: Wear tracks of Stellite 21 under $50 \mathrm{rpm}$ continuous testing (a) $15 \mathrm{~N}$ for $10 \mathrm{hrs}$, (b) $15 \mathrm{~N}$ for $20 \mathrm{hrs}$, (c) $15 \mathrm{~N}$ for $40 \mathrm{hrs,} \mathrm{(d)} 25 \mathrm{~N}$ for $10 \mathrm{hrs,} \mathrm{(e)} 25 \mathrm{~N}$ for $20 \mathrm{hrs}$, (f) $25 \mathrm{~N}$ for $40 \mathrm{hrs}$. 


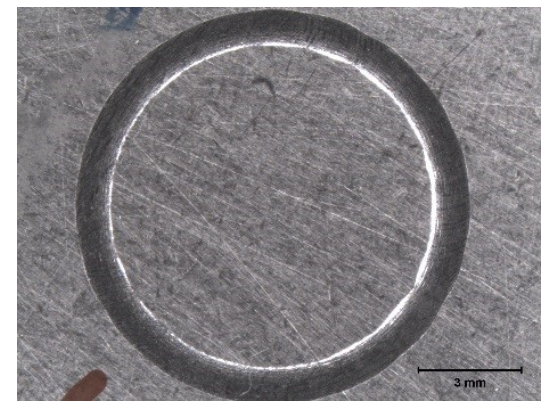

(a)

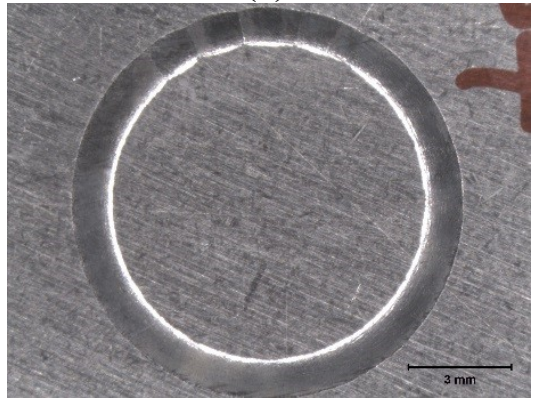

(d)

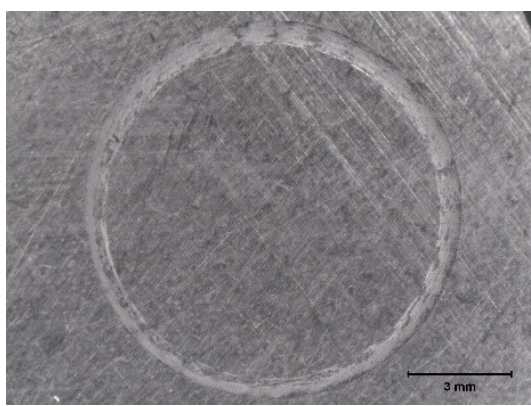

(b)

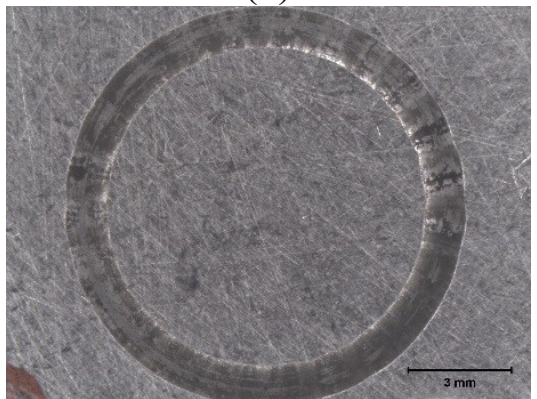

(e)

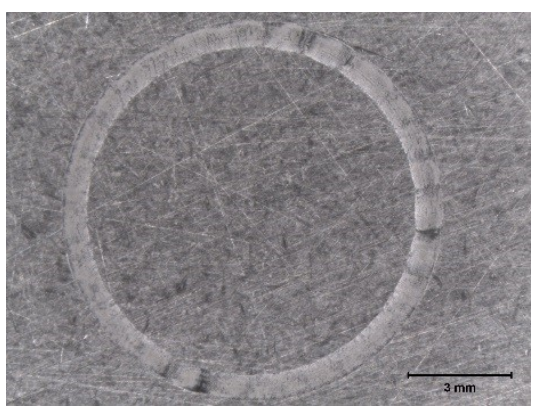

(c)

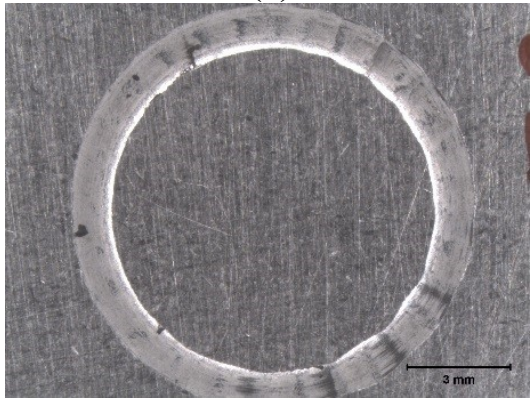

(f)

Figure 4-8: Wear tracks of Stellite 720 under $50 \mathrm{rpm}$ continuous testing at (a) $15 \mathrm{~N}$ for $10 \mathrm{hrs,}$ (b) $15 \mathrm{~N}$ for $20 \mathrm{hrs}$, (c) $15 \mathrm{~N}$ for $40 \mathrm{hrs}$, (d) $25 \mathrm{~N}$ for $10 \mathrm{hrs}$, (e) $25 \mathrm{~N}$ for $20 \mathrm{hrs}$, (f) 25 $\mathrm{N}$ for $40 \mathrm{hrs}$.

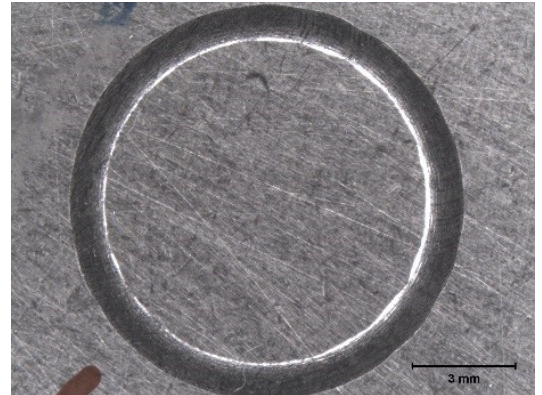

(a)

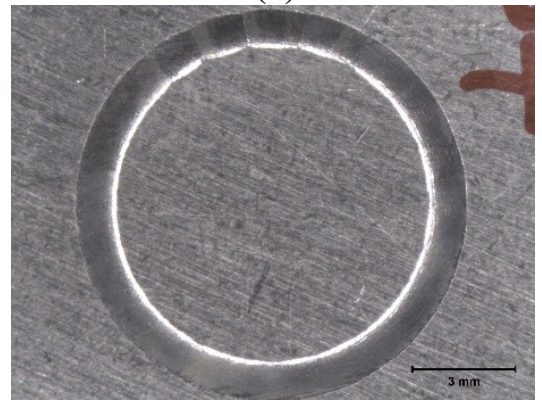

(d)

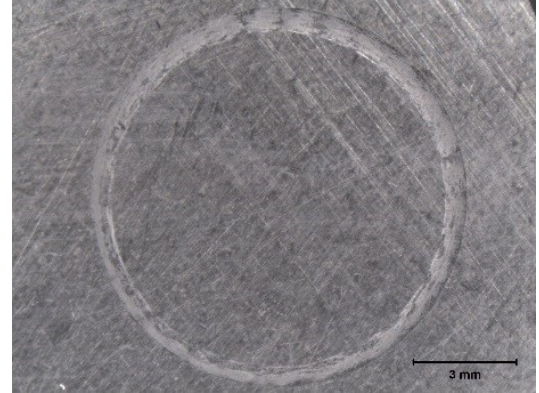

(b)

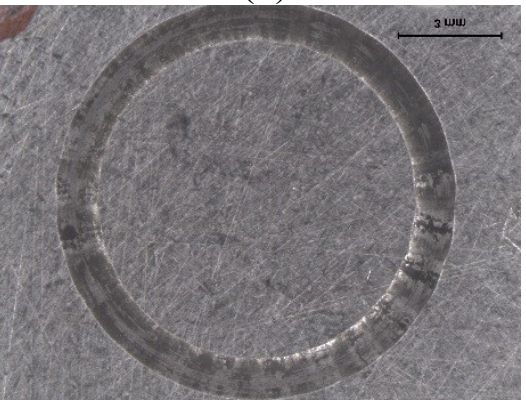

(e)

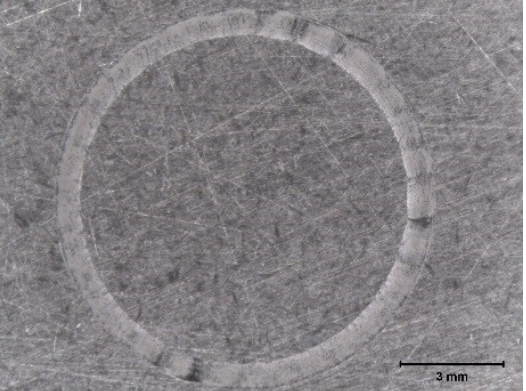

(c)

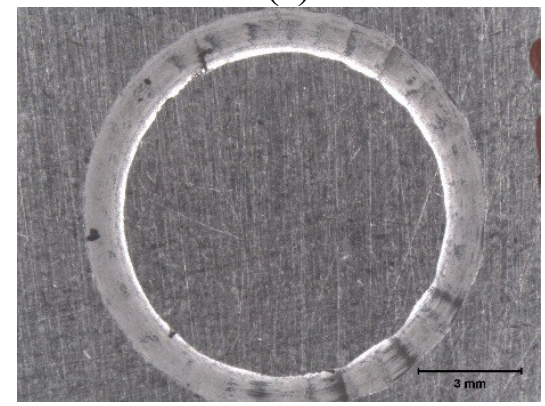

(f)

Figure 4-9: Wear tracks of Stellite 728 under $50 \mathrm{rpm}$ continuous testing at (a) $15 \mathrm{~N}$ for $10 \mathrm{hrs,}$ (b) $15 \mathrm{~N}$ for $20 \mathrm{hrs,} \mathrm{(c)} 15 \mathrm{~N}$ for $40 \mathrm{hrs,} \mathrm{(d)} 25 \mathrm{~N}$ for $10 \mathrm{hrs}$, (e) $25 \mathrm{~N}$ for $20 \mathrm{hrs,} \mathrm{(f)} 25$ $\mathrm{N}$ for 40 hrs. 


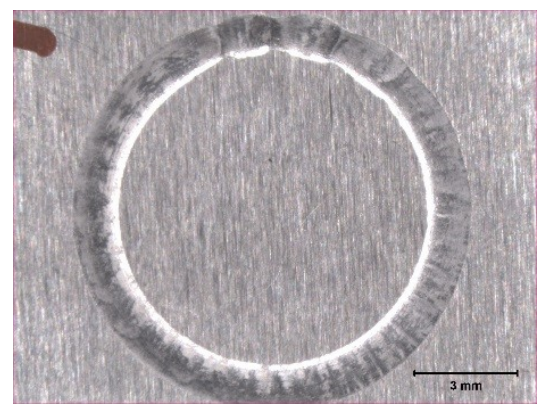

(a)

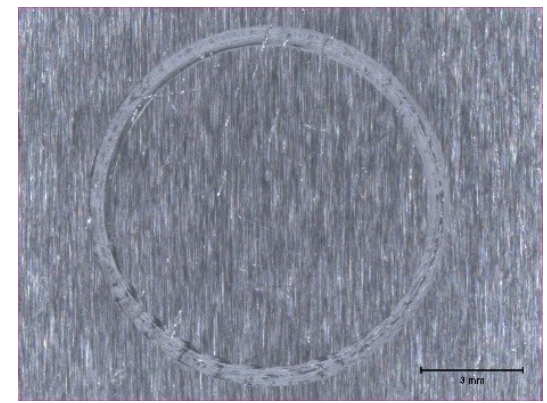

(b)

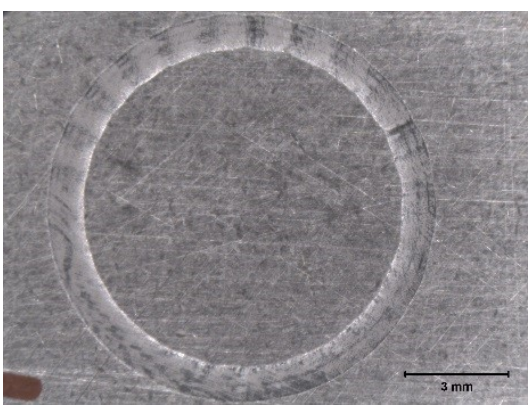

(c)

Figure 4-10: Wear tracks for $50 \mathrm{rpm}$ interrupted testing under $25 \mathrm{~N}$ load for (a) Stellite 21 for time intervals up to $40 \mathrm{hrs}$, (b) Stellite 720 for time intervals up to $40 \mathrm{hrs}$, (c) Stellite 728 for time intervals up to $40 \mathrm{hrs}$.

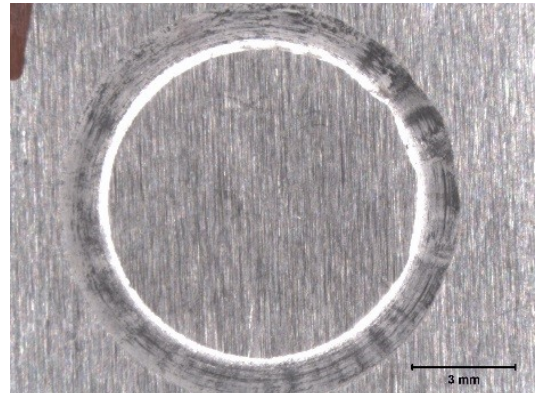

(a)

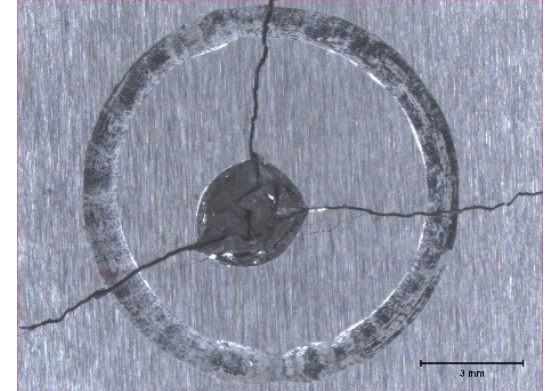

(b)

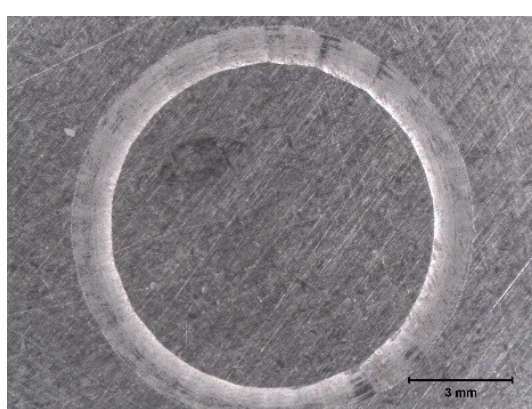

(c)

Figure 4-11: Wear tracks for $60 \mathrm{rpm}$ interrupted testing under $25 \mathrm{~N}$ load for (a) Stellite 21 for time intervals up to $40 \mathrm{hrs}$, (b) Stellite 720 for time intervals up to $40 \mathrm{hrs}$, cracking due to hardness test overloading (c) Stellite 728 for time intervals up to $40 \mathrm{hrs}$.

\subsection{Wear Volume Loss}

\subsubsection{Wear Track Analysis}

The wear resistance of a material can be estimated via the volume loss experienced by the

material. The surface profile measuring system was used to measure the loss on each specimen via a four-point measuring analysis on the wear track, as illustrated in Figure 4-12. An example of one data point (cross-section profile of the wear track) is shown in Figure 4-13. 


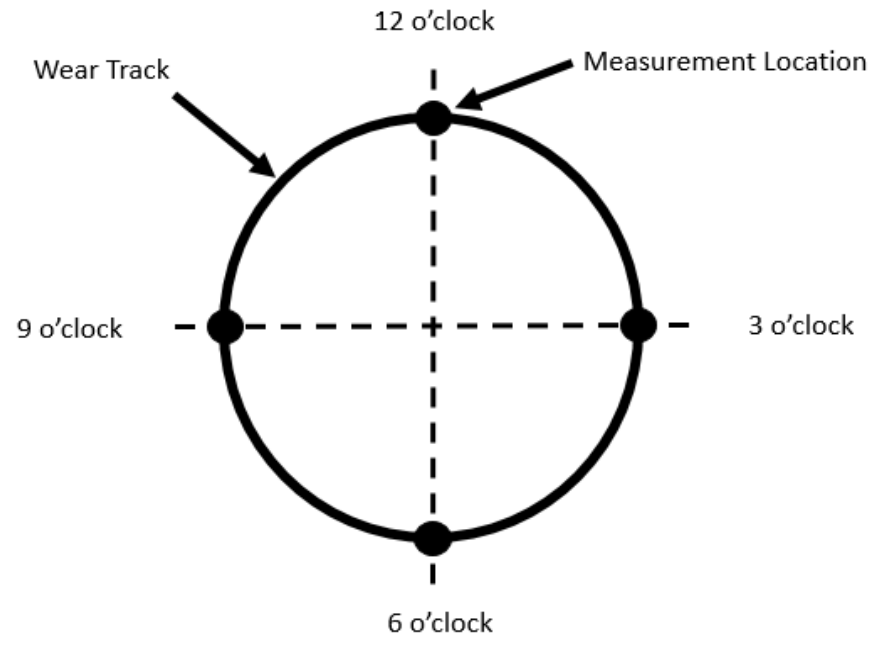

Figure 4-12: Locations of four measurement points on wear track.

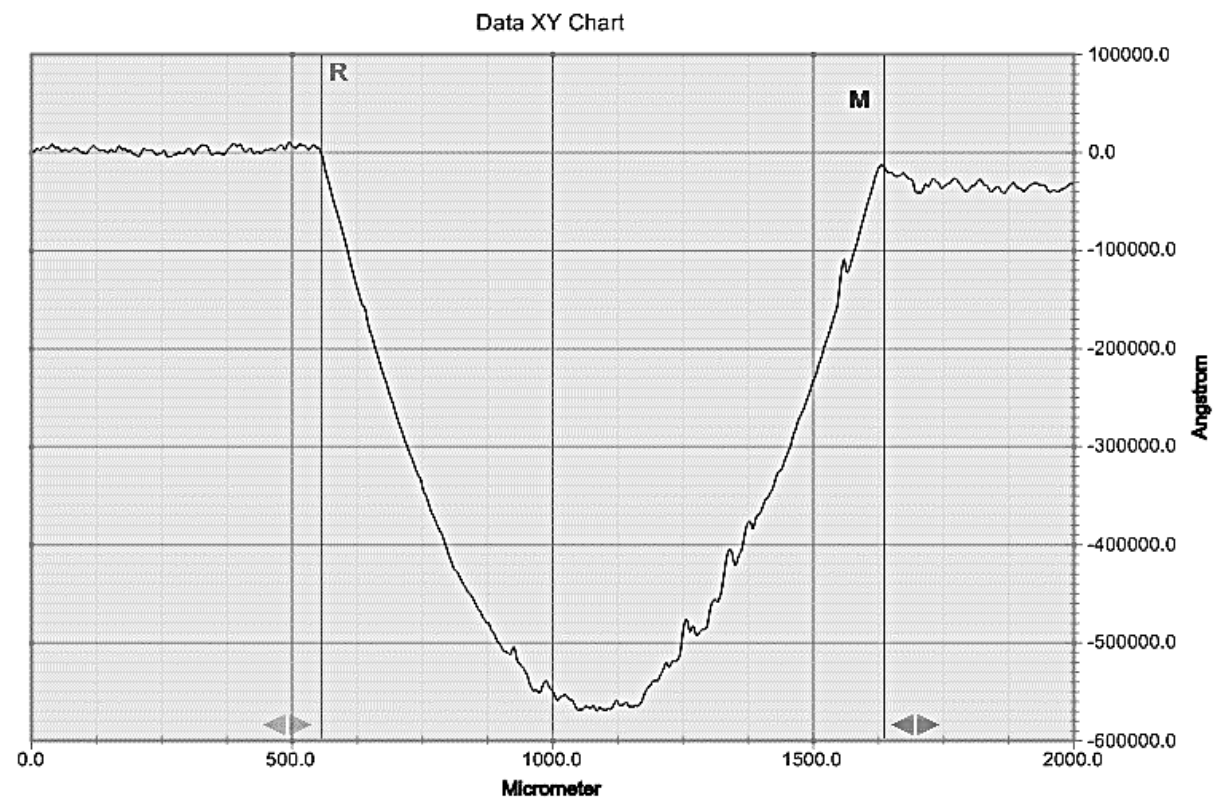

Figure 4-13: An example of wear track cross-section profilometer measurement.

An average was taken of the measurement results of the four points to find the average cross-sectional area on the cross-section of the wear track. This value was then multiplied by the circumference $(\pi d, d=10 \mathrm{~mm})$, to obtain the volume loss $\left(\mathrm{mm}^{3}\right)$ of the circumferentially shaped wear track. It should be noted that the wear tracks are not perfectly circular in width, as seen in 
Figure 4-7 to Figure 4-11. The wear tracks all show varying degrees of width, and subsequent depth changes around the periphery. In some cases, a "worm like" surface texture has developed where high and low points converge (Figure 4-8f). However, four points should adequately capture the wear track cross-section along the periphery of the wear track considering that the specimens did not show out of round features, i.e., a preferential wear on one side of the wear track from misalignment.

\subsubsection{Wear Loss from Continuous Testing at $50 \mathrm{rpm}$ under $15 \mathrm{~N}$ and $25 \mathrm{~N}$}

The overall volume loss was calculated for each specimen (Table 4-1) and is presented as volume loss over the distance travelled (Figure 4-14).

Table 4-3: Volume loss results for continuous testing

\begin{tabular}{|c|c|c|c|c|}
\hline Alloy & $\begin{array}{c}\text { Force } \\
\text { (N) }\end{array}$ & $\begin{array}{c}\text { Time } \\
\text { (hr) }\end{array}$ & $\begin{array}{c}\text { Distance } \\
(\mathbf{k m})\end{array}$ & Volume loss $\left(\mathrm{mm}^{3}\right)$ \\
\hline \multirow{6}{*}{ Stellite 21} & \multirow{3}{*}{15} & 10 & 0.9426 & 0.939 \\
\hline & & 20 & 1.8852 & 0.815 \\
\hline & & 40 & 3.7704 & 1.338 \\
\hline & \multirow{3}{*}{25} & 10 & 0.9426 & 1.782 \\
\hline & & 20 & 1.8852 & 4.541 \\
\hline & & 40 & 3.7704 & 6.155 \\
\hline \multirow{6}{*}{ Stellite 720} & \multirow{3}{*}{15} & 10 & 0.9426 & 0.059 \\
\hline & & 20 & 1.8852 & 0.325 \\
\hline & & 40 & 3.7704 & 0.058 \\
\hline & \multirow{3}{*}{25} & 10 & 0.9426 & 0.342 \\
\hline & & 20 & 1.8852 & 0.384 \\
\hline & & 40 & 3.7704 & 0.369 \\
\hline \multirow{6}{*}{ Stellite 728} & \multirow{3}{*}{15} & 10 & 0.9426 & 0.979 \\
\hline & & 20 & 1.8852 & 0.212 \\
\hline & & 40 & 3.7704 & 0.382 \\
\hline & \multirow{3}{*}{25} & 10 & 0.9426 & 1.106 \\
\hline & & 20 & 1.8852 & 1.448 \\
\hline & & 40 & 3.7704 & 1.418 \\
\hline
\end{tabular}




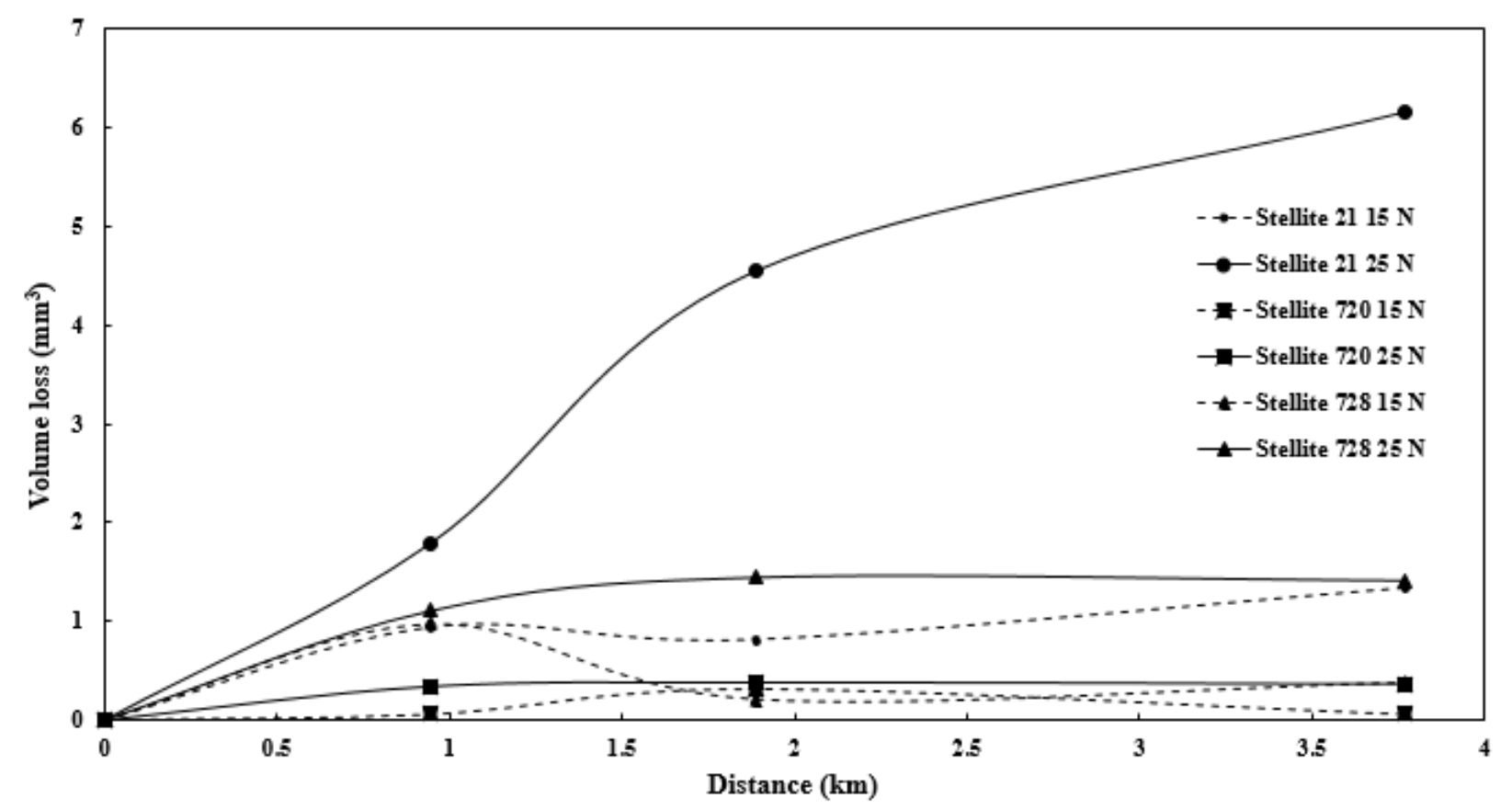

Figure 4-14: Combined volume loss at $50 \mathrm{rpm}$ under $15 \mathrm{~N}$ and $25 \mathrm{~N}$ continuous testing.

As shown in Figure 4-14, under $15 \mathrm{~N}$ the specimens show a very minor volume loss across all three alloys. The Stellite 21 specimen at $25 \mathrm{~N}$ shows a significant volume loss compared to Stellite 720 and Stellite 728 that appear to be quite consistent with respect to the distance, and do not show a significant volume loss $\left(<1.5 \mathrm{~mm}^{3}\right)$ over all the durations under both $15 \mathrm{~N}$ and $25 \mathrm{~N}$ loads. All the measurement standard deviation data is presented in the Appendix.

The significant transition appears to be around the $1 \mathrm{~km}$ marker, after this point the specimens do not show significant increase of volume losses for most of the specimens tested, except Stellite 21 under the higher load. This marker is the first initial measurement for the continuous testing specimens, therefore the zero to $1 \mathrm{~km}$ is a projected value for volume loss. This transition instigated the thought to look at the interrupted testing methodology. 


\subsubsection{Wear Volume Loss from Interrupted Testing at $50 \mathrm{rpm}$ under $25 \mathrm{~N}$}

Interrupted testing at $50 \mathrm{rpm}$ under a normal load of $25 \mathrm{~N}$ was carried out to obtain numerous data points in order to build a profile of the wear loss. A normal load of $25 \mathrm{~N}$ was chosen based on the preliminary data shown in Figure 4-14. A $15 \mathrm{~N}$ load did not show significant changes between the alloys in terms of volume loss. Table 4-4 and Figure 4-15 present the data obtained from the interrupted testing of the three Stellite alloys. 
Table 4-4: Volume loss results for interrupted testing

\begin{tabular}{|c|c|c|c|c|}
\hline Alloy & Force (N) & Time interval (hr) & Distance (km) & Volume loss $\left(\mathrm{mm}^{3}\right)$ \\
\hline \multirow{9}{*}{21} & \multirow{9}{*}{25} & 1 & 0.09426 & 0.148 \\
\hline & & 3 & 0.28278 & 0.399 \\
\hline & & 5 & 0.4713 & 0.701 \\
\hline & & 7 & 0.65982 & 1.024 \\
\hline & & 11 & 1.03686 & 1.860 \\
\hline & & 15 & 1.4139 & 1.705 \\
\hline & & 20 & 1.8852 & 1.879 \\
\hline & & 30 & 2.8278 & 1.847 \\
\hline & & 40 & 3.7704 & 1.928 \\
\hline \multirow{9}{*}{720} & \multirow{9}{*}{25} & 1 & 0.09426 & 0.102 \\
\hline & & 3 & 0.28278 & 0.146 \\
\hline & & 5 & 0.4713 & 0.125 \\
\hline & & 7 & 0.65982 & 0.144 \\
\hline & & 9 & 0.84834 & 0.138 \\
\hline & & 11 & 1.03686 & 0.150 \\
\hline & & 15 & 1.4139 & 0.144 \\
\hline & & 17 & 1.60242 & 0.140 \\
\hline & & 40 & 3.7704 & 0.152 \\
\hline \multirow{11}{*}{728} & \multirow{11}{*}{25} & 1 & 0.09426 & 0.094 \\
\hline & & 3 & 0.28278 & 0.263 \\
\hline & & 5 & 0.4713 & 0.460 \\
\hline & & 7 & 0.65982 & 0.640 \\
\hline & & 9 & 0.84834 & 0.857 \\
\hline & & 11 & 1.03686 & 0.854 \\
\hline & & 13 & 1.22538 & 0.873 \\
\hline & & 15 & 1.4139 & 0.874 \\
\hline & & 17 & 1.60242 & 0.651 \\
\hline & & 30 & 2.8278 & 0.875 \\
\hline & & 40 & 3.7704 & 0.911 \\
\hline
\end{tabular}


An evident difference in volume loss between the alloys can be identified. The continuous tests to finite distances generate few data points for studying the wear-loss mechanism and behaviour of the alloys, especially during the transient period. Figure 4-15 clearly shows a trend that the majority of volume loss occurs in the first $1.5 \mathrm{~km}$ for Stellite 21 and Stellite 728 under the interrupted test condition. As expected, Stellite 21 having a minimal volume fraction of carbides $(\sim 4 \%)$ has the highest volume loss, whereas Stellite 720 having the highest volume fraction $(\sim 70$ \%) has minimal volume loss, and Stellite 728 (21\%) falls between Stellite 21 and Stellite 720 in terms of volume loss.

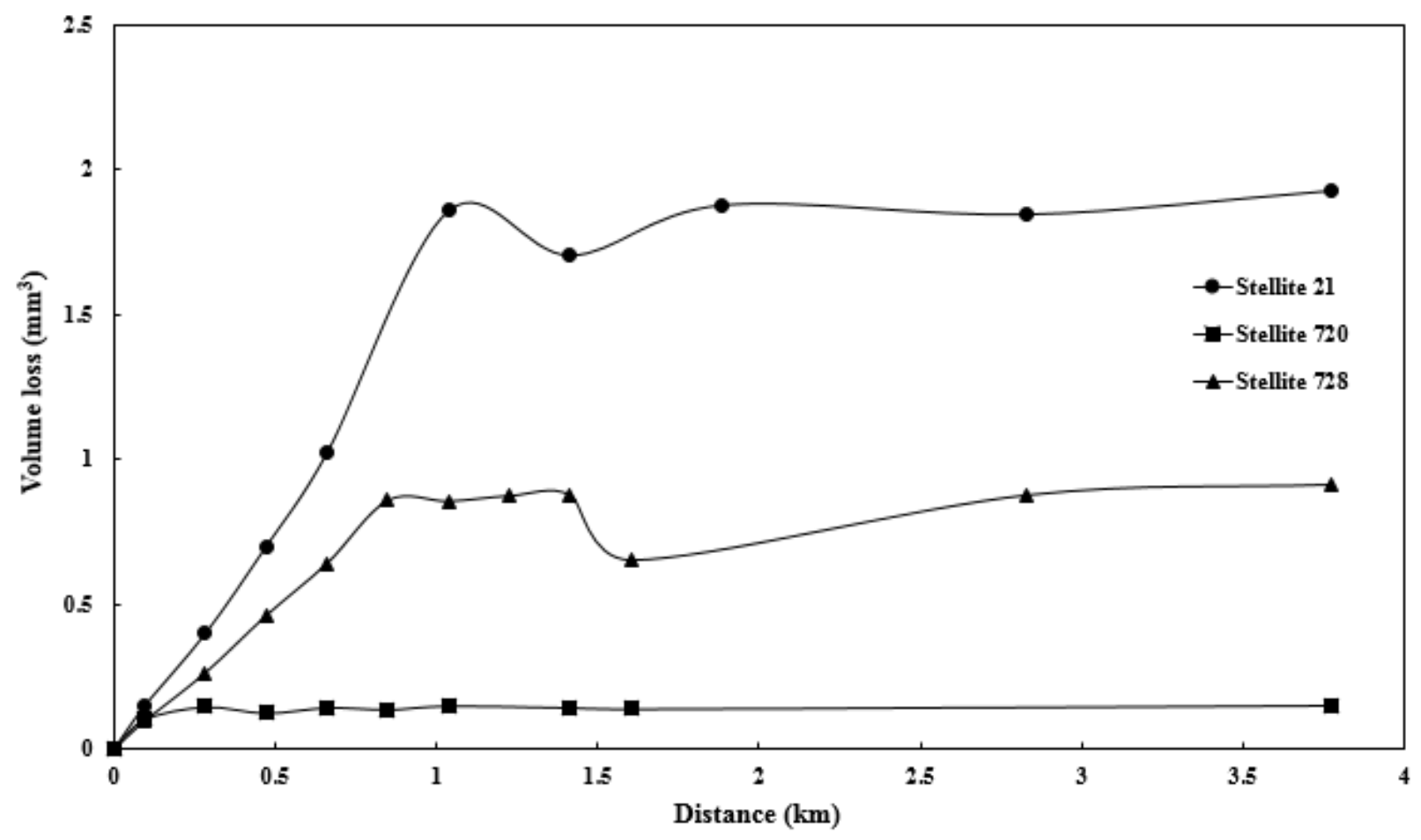

Figure 4-15: Volume loss for interrupted testing at $50 \mathrm{rpm}$ under $25 \mathrm{~N}$.

\subsubsection{Comparison of Interrupted and Continuous Testing at $50 \mathrm{rpm}$ under $25 \mathrm{~N}$}

The individual differences between the continuous test and the interrupted testing for each alloy are presented in Figure 4-16. The longer duration test results show a relatively significant 
difference for each of the alloys, and they all show an increase in volume loss for the continuous specimens over the interrupted ones. Taking into consideration the removal of the specimen during the interrupted testing, and the falling-off of the wear debris during measurement, this will influence changes in the overall wear system in terms of particles being available to act as the third-body participating in wear. However, the changes do not become apparent until $\sim 0.8 \mathrm{~km}$ duration. This suggests that wear in the initial stages is similar even whether the wear debris is present or not. However, over longer durations, the loose debris contributes significantly to the wear of the surface causing a higher volume loss in the continuous specimens.

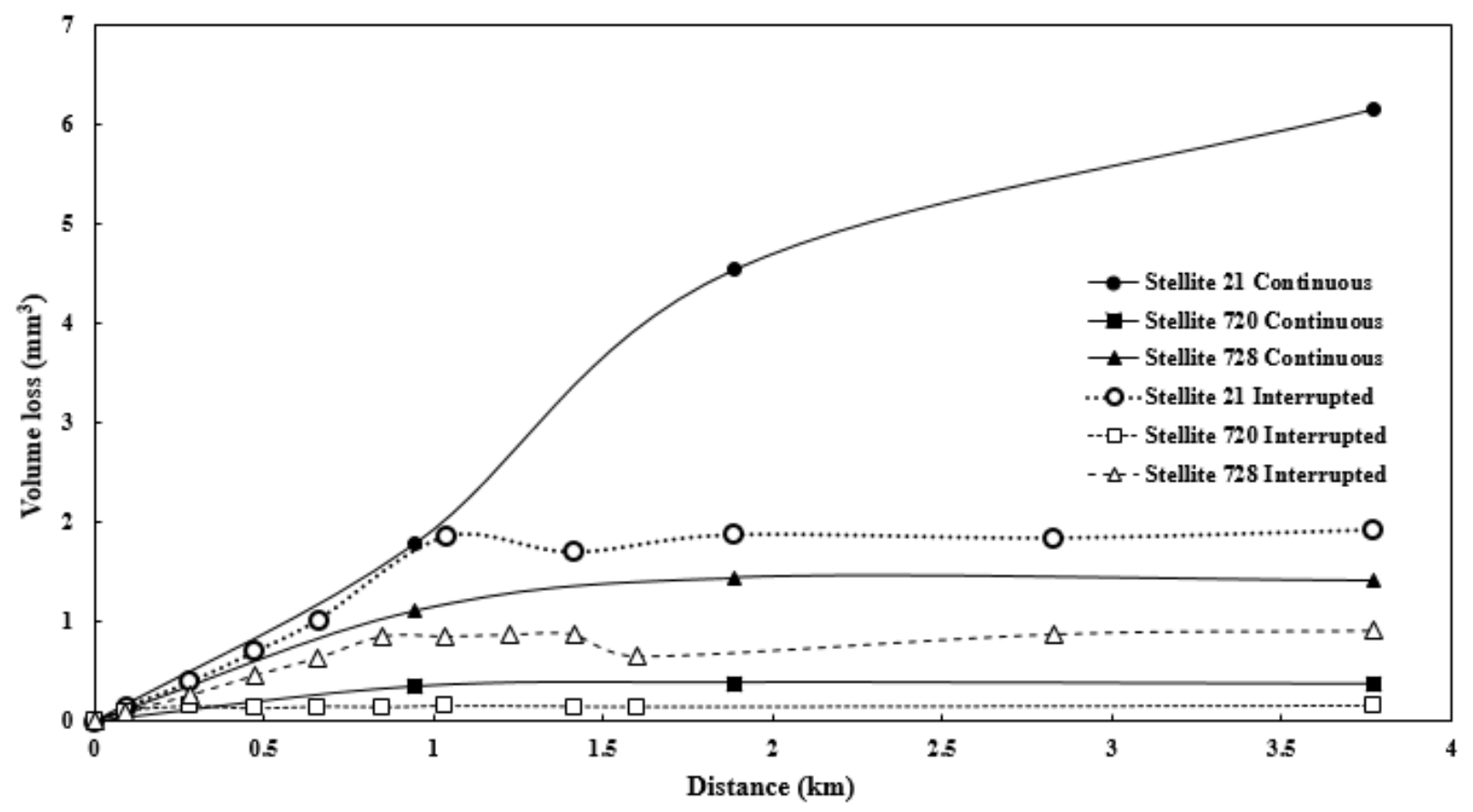

Figure 4-16: Volume loss of continuous vs. interrupted testing under $25 \mathrm{~N}$ at $50 \mathrm{rpm}$. 


\subsubsection{Wear Loss of Interrupted Testing at $50 \mathrm{rpm}$ and $60 \mathrm{rpm}$ under $25 \mathrm{~N}$}

A test at $60 \mathrm{rpm}$ under $25 \mathrm{~N}$ was conducted at similar intervals as those for the tests at 50 rpm under $25 \mathrm{~N}$. The obtained data are presented in Figure 4-17, which show similar trends for Stellite 21 and Stellite 728. However, Stellite 720 shows an increased volume loss at the higher rpm suggesting that a more rapid speed was affecting the breakdown of the oxide layer and/or the breakdown of the carbides, which in turn would expose the solid solution to attack by losing the protection of the carbides.

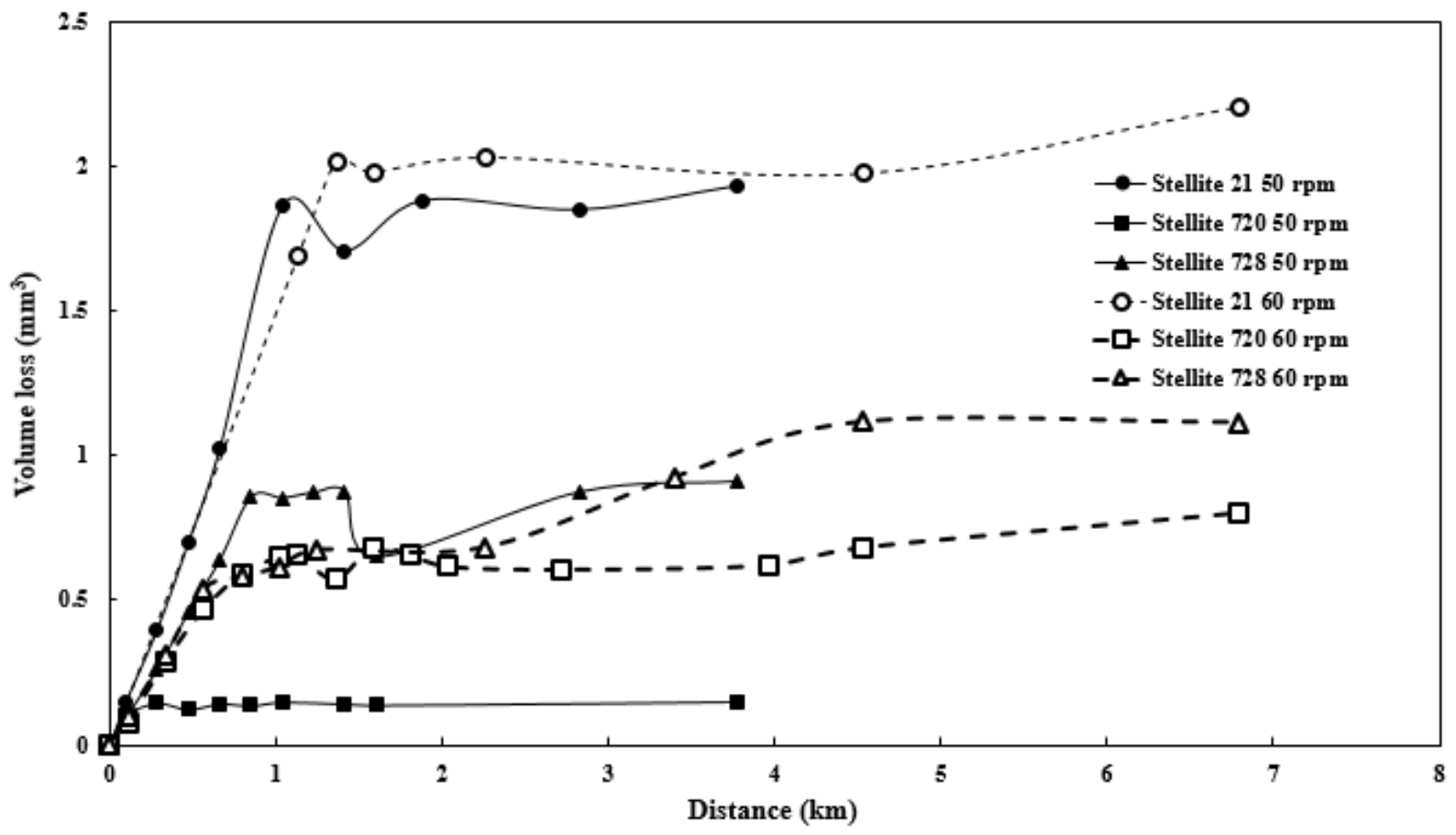

Figure 4-17: Volume loss during interrupted testing at $50 \mathrm{rpm}$ and $60 \mathrm{rpm}$ under $25 \mathrm{~N}$.

\subsection{Friction Coefficient}

Friction coefficient is defined by the measure of friction force between two contacting objects under a normal force. Usually, the rougher the surfaces in contact with each other the higher the value of the friction coefficient is. The software and sensors on the pin-on-disc 
tribometer generate friction coefficient data during testing. The raw data are processed to look at the effect of friction coefficient by calculating the average friction coefficient for the duration of the testing.

\subsubsection{Friction Coefficient Analysis for Continuous Testing at $50 \mathrm{rpm}$ under $15 \mathrm{~N}$ and $25 \mathrm{~N}$}

The average friction coefficients for the continuous wear tests were calculated by taking the sum of the entire data set and averaging and the results are presented in Figure 4-18. Some variation in the data can be identified at the individual points. However, all three Stellite alloys appear to have a similar trend and value for the average friction coefficient at $15 \mathrm{~N}$. At $25 \mathrm{~N}$, the data points spread out slightly to show that Stellite 21 has a reduced average friction coefficient. This indicates a smoother wear track, which is to be expected from this alloy having a small volume fraction of hard phases and mostly a softer solid solution to breakdown in the wear path.

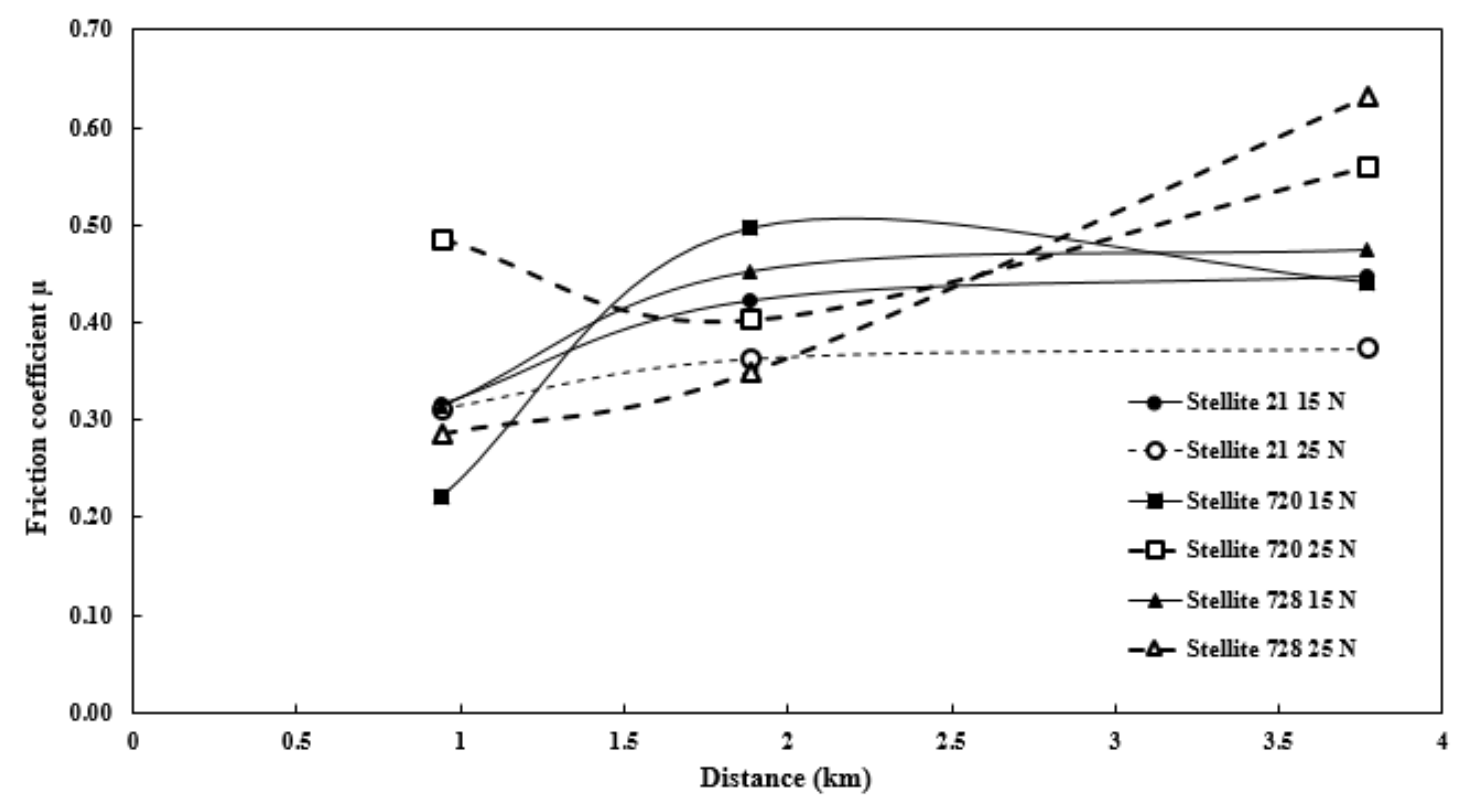

Figure 4-18: Average friction coefficient for continuous testing at $50 \mathrm{rpm}$ under $15 \mathrm{~N}$. 


\subsubsection{Friction Coefficient Analysis Comparison of Interrupted and Continuous}

\section{Testing at $50 \mathrm{rpm}$ under $25 \mathrm{~N}$}

The friction coefficient can represent changes in the topography of the wear surface during testing, which, in the case of the interrupted testing, is of significant importance due to the removal of wear debris. A change in the wear system can be identified when looking at the average wear coefficient differences between continuous and interrupted testing (Figure 4-19). To calculate the friction coefficient for the interrupted testing, the average coefficient of friction was cumulatively added to create the trend presented in Figure 4-19.

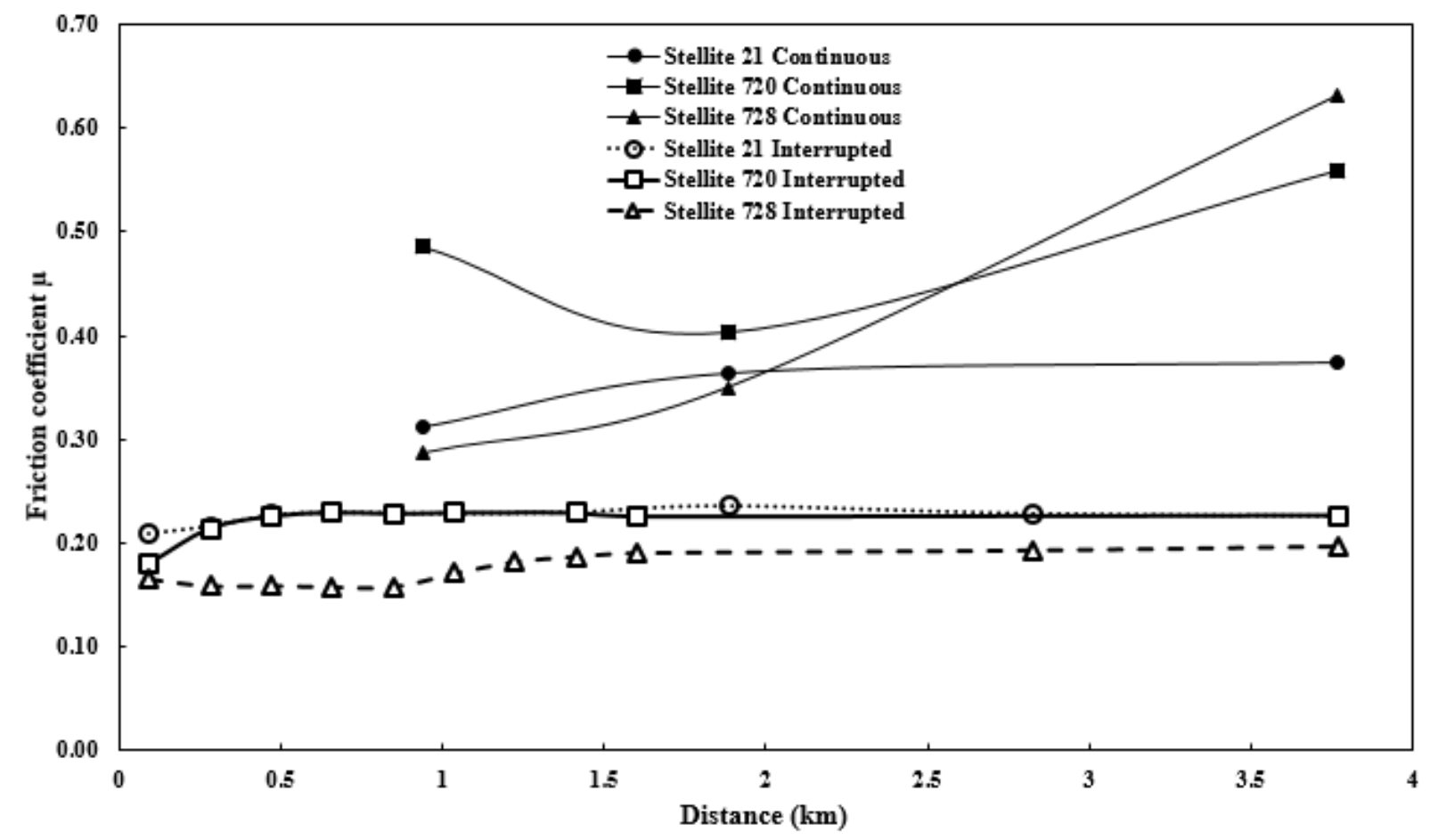

Figure 4-19: Friction coefficient of continuous vs. interrupted testing at $50 \mathrm{rpm}$ under $25 \mathrm{~N}$.

From Figure 4-19, it can be observed that the interrupted test specimens have a lower average friction coefficient than the continuous test ones. This was expected due to the removal of debris creating a smoother wear path. The average friction coefficient for the interrupted test 
specimens also shows a relatively flat linear profile as the distance increases, another feature of the debris removal.

The continuous testing data show that Stellite 21 has an overall lower coefficient of friction, which is to be expected due to the lack of carbides and a softer solid soution forming the bulk material loss. However, Stellite 728 shows a lower overall coefficent of friction for the interrupted testing. The actual value is negligible between the interrupted data sets, but this provides some insight to the beahviour of Stellite 728 .

\subsubsection{Friction Coefficient Comparison of Interrupted Testing at $50 \mathrm{rpm}$ and 60 rpm under $25 \mathrm{~N}$}

The interrupted testing at $50 \mathrm{rpm}$ and $60 \mathrm{rpm}$ showed a steady trend in friction coefficient values. Figure 4-20 shows a similar trend for both sets of data that proved to be quite flat compared to the changes seen in the continuous testing. The increase in rotation speed resulted in an increase in the friction coefficient for all the three alloys.

Stellite 21 would generally be considered to have the lowest friction coefficient from the limited number of carbides and mostly the softer solid solution forming the bulk of the wear debris. However, one interesting point to note is that Stellite 728 shows the lowest coefficient of friction for both sets of data. As previously discussed, the lowest friction coefficient value would be expected from Stellite 21. Stellite 728 has more carbides than Stellite 21 but the carbides have a different form so may not break down easily leaving mostly solid solution in the wear path. It would be interesting to evaluate the hardness of the matrix (solid solution) of both Stellite 21 and Stellite 728 to ascertain if there is a difference between the two alloys. 


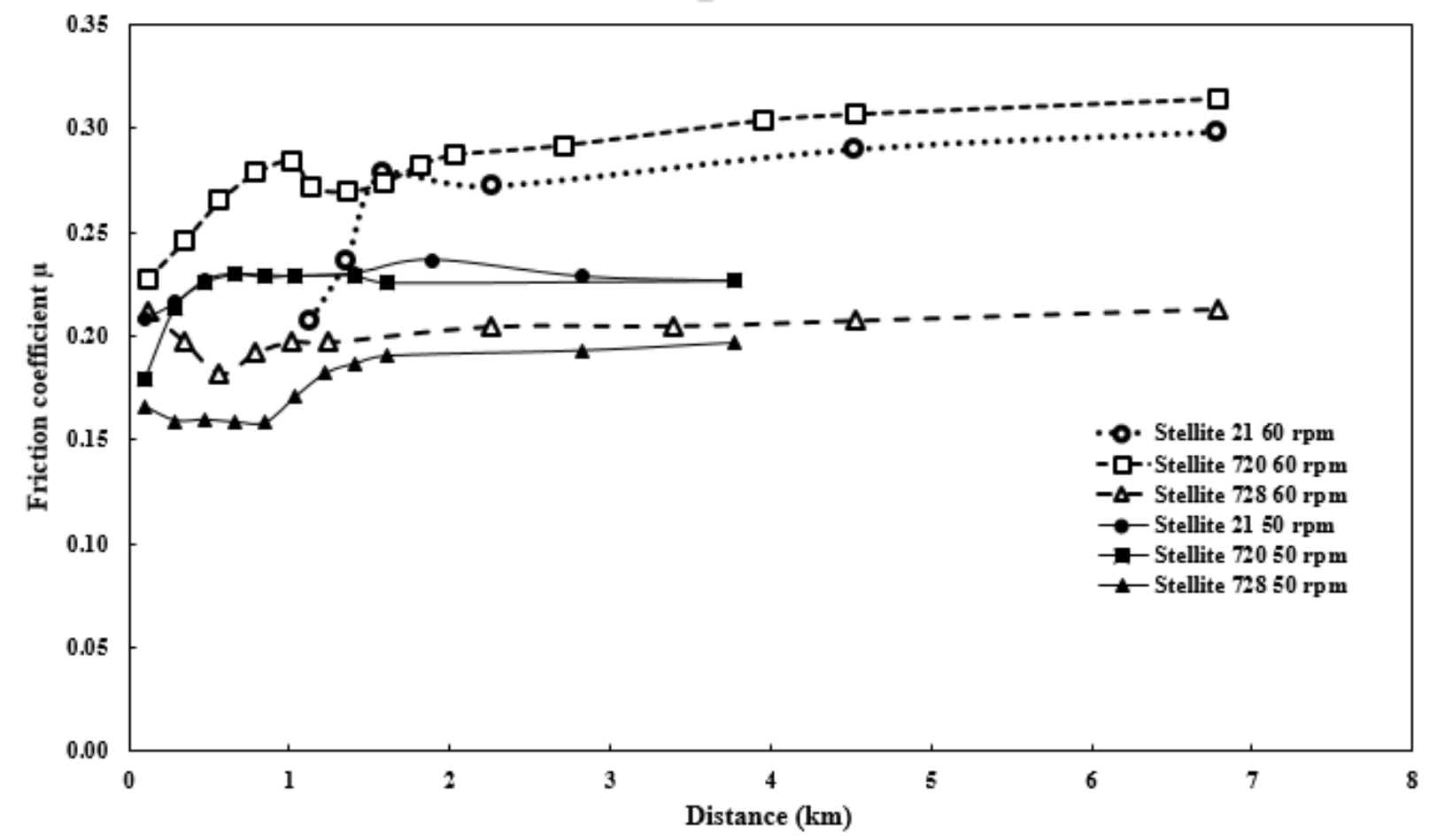

Figure 4-20: Friction coefficient for interrupted testing at $50 \mathrm{rpm}$ and $60 \mathrm{rpm}$ under $25 \mathrm{~N}$.

\subsection{Wear Data Analysis}

\subsubsection{Steady-State Wear and the Archard Equation}

In attempting to model the molybdenum Stellite alloys to predict wear (volume) loss, the literature review indicates that there are many variables associated with sliding wear. A fundamental equation, which can be considered a starting point to modeling sliding wear is the Archard equation [21]. However, this equation contains a variable for distance covered, but does not include a time factor. In understanding the wear process, the distance covered over a particular time, i.e, speed, can be of significant importance. In addition, the Archard equation only applies when steady-state conditions are present. Steady-state (surface equilibrium) can be identified by a steady increase in volume loss over the number of cycles/time/distance, as shown in Figure 2-18. The volume loss graphs in the present research show this steady-state, particularly for the 
continuous testing on Stellite 21 at $25 \mathrm{~N}$ (Figure 4-14). The steady state is represented by the almost linear volume loss as the duration increases. In contrast, the interrupted testing data shown in Figure 4-15 shows a steady-state for all three alloys. However, the volume loss is consistently low as the duration increases which makes the line more horizontal than linear.

The raw data presented in Figure 4-5 include the friction coefficient measurement on one axis. Transitions in the friction coefficient graphs reveal a change in the wear process, i.e. oxide formation and/or debris forming in the wear path. However, the graphs directly generated from the wear testing show an immense amount of fluctuations as shown in Figure 4-5. This demonstrates the variability in the testing, and how it is important to take into account the different ways to evaluate the data.

It is necessary to recognize that the different data sets as well as the final result of volume loss can generate a picture of the events occurring during the wear process. For example, if the friction coefficient suddenly increases, this likely indicates a surface oxide breaking down and moving into the wear path as debris causes the friction force to increase. However, a corresponding volume loss measurement can show a minimal change because the oxide layer can be quite thin. Therefore, both sets of data have value in interpreting the results.

\subsubsection{Wear Coefficient}

Another interpretation of data can be calculating the wear coefficient. This value is a simple estimate of how a system responds to wear. As with most wear processes, there is a wear-in stage at the beginning which has a dynamic changing wear rate and is challenging to predict. However, once a steady-state has been established, the wear coefficient can be used as a guide to comparing the wear losses that can potentially be experienced by materials. This has a particular use in 
considering materials during the design stages. In the present research, the Archard equation was employed to calculate the wear coefficient per unit sliding distance:

$$
w=\frac{K P}{H}
$$

where $w$ is the worn volume $\left(\mathrm{mm}^{3}\right.$ per unit distance), $K$ is referred to as the wear coefficient (or, coefficient of wear), $P$ is the applied load $(\mathrm{N})$, and $H$ is the hardness of the worn material (HRC). The Archard equation is considered to be a simple model for the steady-state zone of sliding wear.

In Eq. (4.1), the hardness and normal load are constants, and the variable, $s$, which is distance, can be added to this equation to find the total volume loss. No hardness scale is generally given in literature, but Vickers Hardness appears to be a means to represent the hardness of the specimens. However, in the case of Stellite alloys, Rockwell Hardness C-Scale (HRC) is the most common scale used to differentiate the alloys. The size of some of the phases may not capture the Vickers diamond indentation, which would not accurately represent the overall hardness of the material. In addition, localized cracking of the Vickers impression also prevents accurate measurements via this technique and converting measurements between scales is not generally considered good practice. Therefore, hardness measurements were taken on the specimens using a Wilson Rockwell Series B2000 hardness testing machine. The results are presented in Table 4-5, and show an average of three readings.

Table 4-5: Hardness test results for the Stellite alloys

\begin{tabular}{|c|c|}
\hline Stellite alloy & Hardness (HRC) \\
\hline Stellite 21 & 28.6 \\
\hline Stellite 720 & 60.5 \\
\hline Stellite 728 & 38.1 \\
\hline
\end{tabular}


The wear coefficient can be calculated by expressing the total volume loss as the volume loss per unit sliding distance. In the present work, the unit sliding distance is per metre. Then $K$, can be calculated as:

$$
K=\frac{w H}{P}
$$

An analysis of the wear coefficient values from the continuous testing at $50 \mathrm{rpm}$ under 15 $\mathrm{N}$ and $25 \mathrm{~N}$ is depicted in Figure 4-21. The data shows that the specimens under $15 \mathrm{~N}$ has a consistently low value of wear coefficient and a trend towards zero. For the test at $50 \mathrm{rpm}$ under $25 \mathrm{~N}$ the wear coefficients show a gradual decrease as the duration is increased. Stellite 21 has a higher wear coefficient, which is to be expected since it has minimal carbides to combat wear.

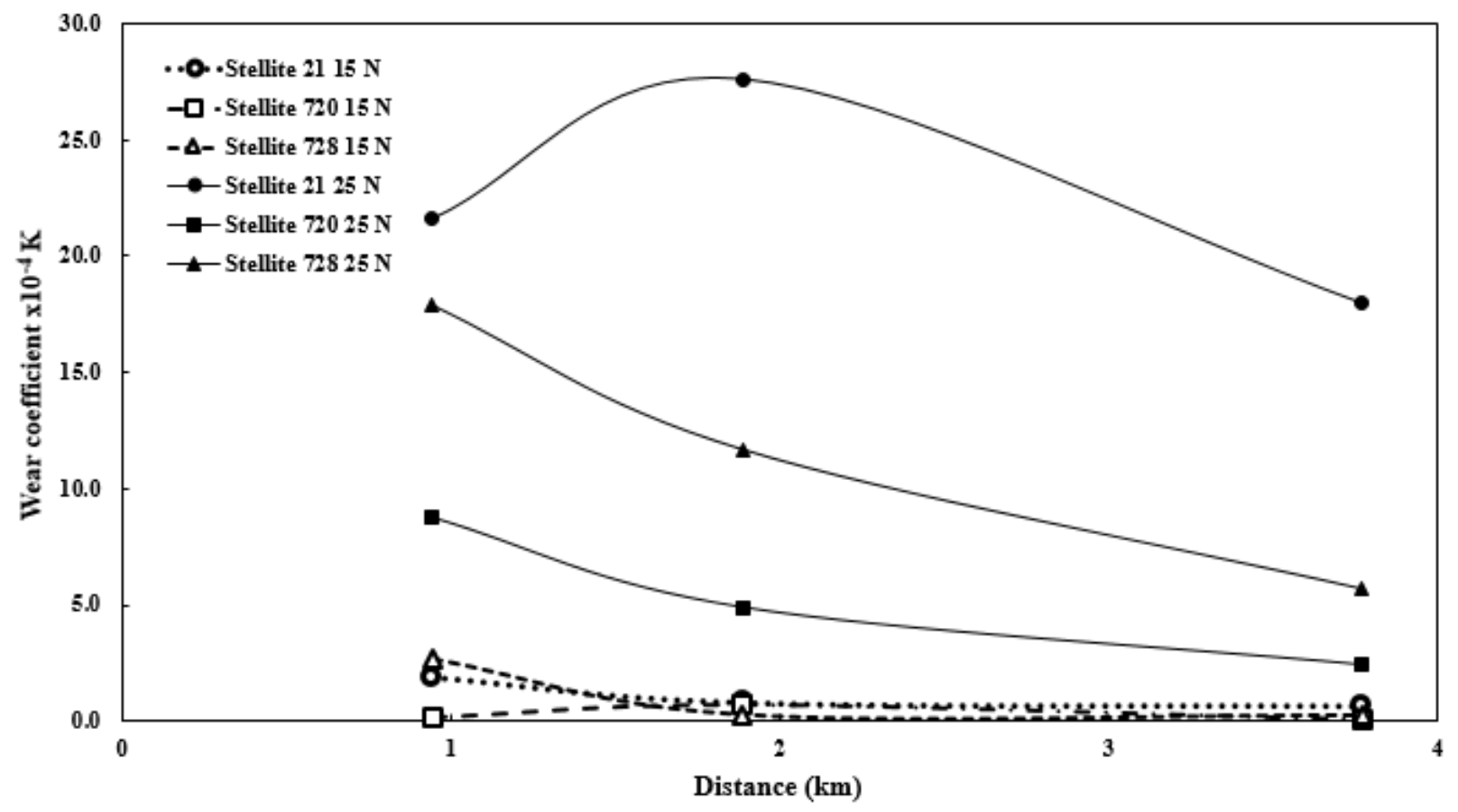

Figure 4-21: Wear coefficient for continuous testing at $50 \mathrm{rpm}$. 
The interrupted testing schedule at $50 \mathrm{rpm}$ captures more data intervals during the testing. These points were also calculated using the Archard equation to find the wear coefficient. Each point has been calculated by using the cumulative volume loss. Figure 4-22 illustrates how the continuous tests at $50 \mathrm{rpm}$ under $25 \mathrm{~N}$ compare to the interrupted test results with the same parameters.

Considering the interrupted testing removes debris and therefore changes the wear system to some degree, Figure 4-22 shows how the wear coefficients for both the continuous and interrupted testing relate to each other. A relatively large difference in friction coefficient is established between the two wear systems. However, except for the continuous test on Stellite 21 under $25 \mathrm{~N}$, the wear coefficient does trend towards zero for the other wear systems.

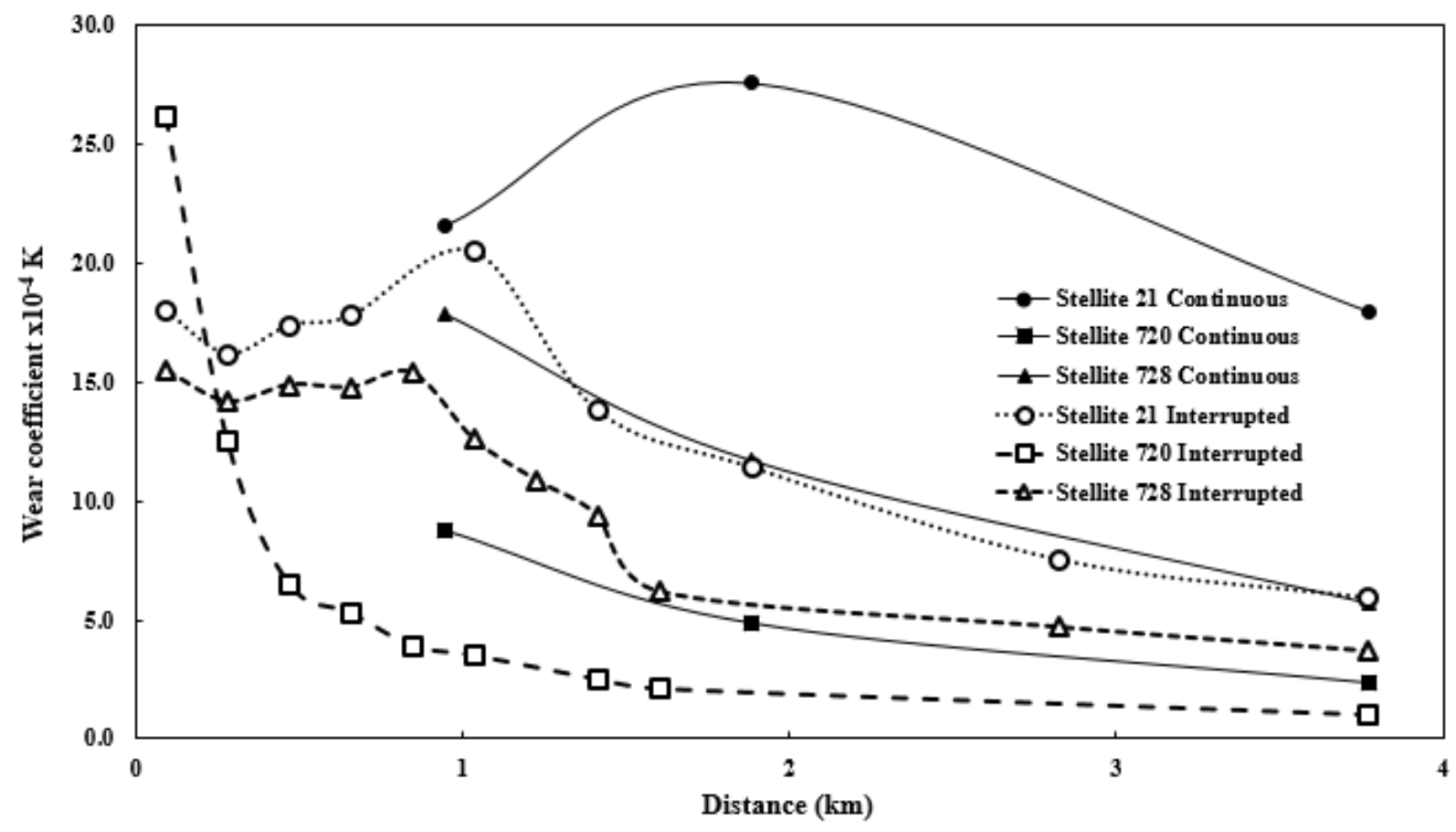

Figure 4-22: Combined interrupted and continuous wear coefficients at $50 \mathrm{rpm}$ under $25 \mathrm{~N}$. 
To compare the interrupted test data for $50 \mathrm{rpm}$ at $25 \mathrm{~N}$, a further interrupted test at $60 \mathrm{rpm}$ under $25 \mathrm{~N}$ was also conducted. Figure 4-23 shows how variable the wear coefficient can be between specimens run with a minimal change in rpm. This would make full modeling to predict volume loss over different speeds and loads extremely challenging. Understanding this wear mechanism is also challenging with the interrupted testing because the wear debris has been removed. It is expected that the abrasive wear on the system would be increased due to the debris being present.

Wear coefficient while providing an approximate number to predict wear loss, also has its challenges because it is essentially a moving target, particularly if the wear debris has been removed from the wear system, which can happen in industrial applications when machines are stopped and cleaned but the parts may not necessarily be replaced. This is an important factor to consider when interpreting the data and thinking about which alloy will perform better under a given set of circumstances.

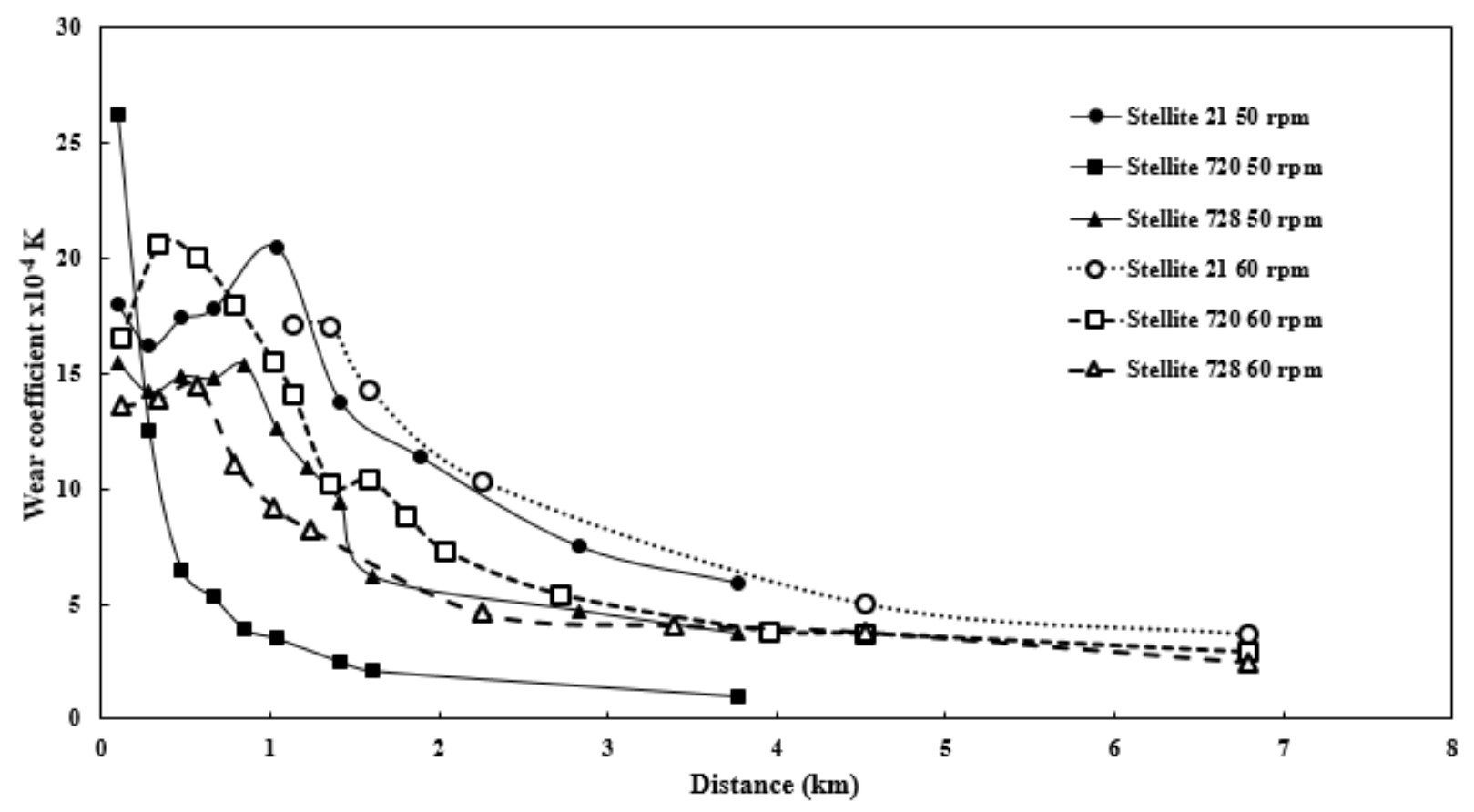

Figure 4-23: Wear coefficient for interrupted testing at $50 \mathrm{rpm}$ and $60 \mathrm{rpm}$ under $25 \mathrm{~N}$. 


\subsection{Analysis of the Worn Surfaces}

In order to interpret the quantitative data either from raw data or calculated, visual examination of the worn surfaces using a Tescan Scanning Electron Microscope (SEM) was carried out. A full peripheral examination was undertaken and representative images of each surface will be presented.

\subsubsection{Worn Surfaces of Stellite 21}

Stellite 21 experienced the most volume loss during the continuous and interrupted testing and this is shown in the SEM image analysis. Figure 4-24 to Figure 4-31 show wide, deep tracks where large areas of material have been removed in bulk. Stellite 21 having the lowest carbon content contains mostly solid solution and very few carbides.

The SEM image at high magnification in Figure 4-24b shows scoring and ploughing of the surface at $10 \mathrm{hrs}$ duration $(0.94 \mathrm{~km})$, and Figure $4-25 \mathrm{~b}$ and Figure $4-25 \mathrm{c}$ for the higher duration tests show a surface layer/debris generated from the wear process. This surface layer shows an accumulated build-up of plastically deformed particles from the cyclic mechanical attack (staircasing profile). This layer is 'electrically' charged during SEM analysis using secondary electron (SE) imaging. Charging means there is a build-up of electrons on the surface because it is not conductive to allow the electrons to flow, and therefore the surface becomes extremely bright. This occurs when the surface being analyzed is detached from the bulk material (thus no grounding via a physical connection), and/or, the surface being analyzed is not conductive. The latter occurs with ceramics and plastic type materials. During wear, even at room temperature, friction heat is generated at the contact surface along the wear track. This localized heat generation will cause high temperature oxidation to occur at the ball/disc interface. Stellite alloys are expected to oxidize 
and the 'charged' layer is the oxidized layer (wear debris) lying on the metal surface. In addition to surface features from wear, the carbide distribution in Stellite alloys is of great importance. Therefore it is important to use both secondary electrons (SEs) to evaluate wear features, and back scatter electrons (BSEs) for analyzing the carbide phases and reducing the charging effects. Primarily SEs were used for Stellite 21 and Stellite 728, and BSEs were used for Stellite 70. However, the individual images display the type of detection method.

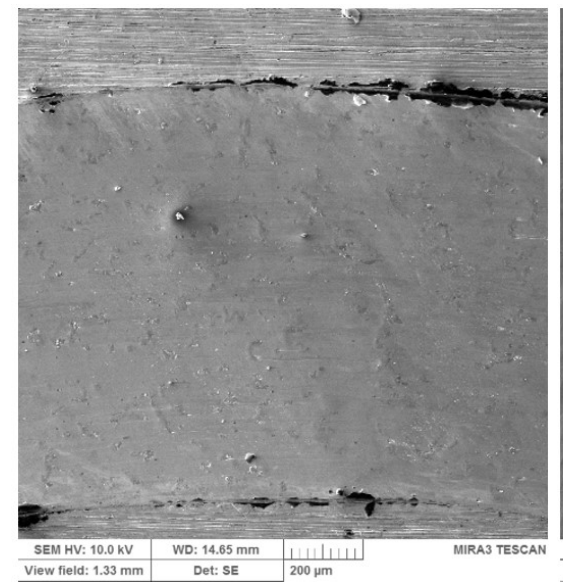

(a)

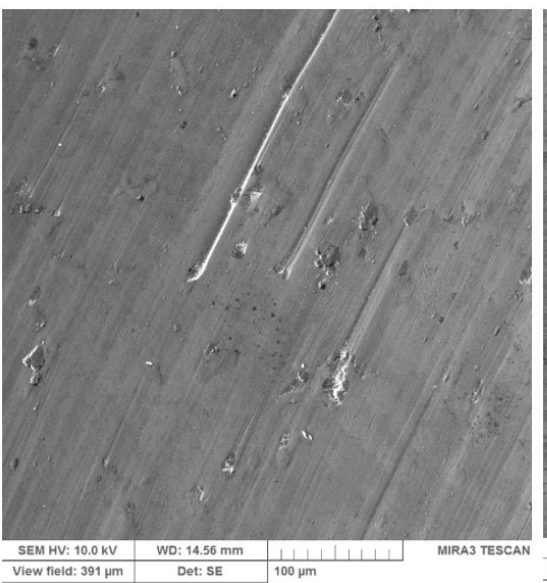

(b)

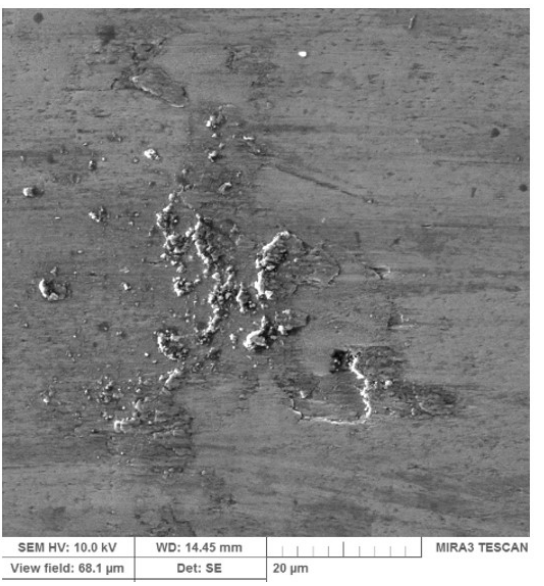

(c)

Figure 4-24: Worn surface of Stellite 21 continuously tested at $50 \mathrm{rpm}$ under $15 \mathrm{~N}$ for $10 \mathrm{hrs}$ (0.94 km) at (a) low magnification, (b) medium magnification, (c) high magnification. 


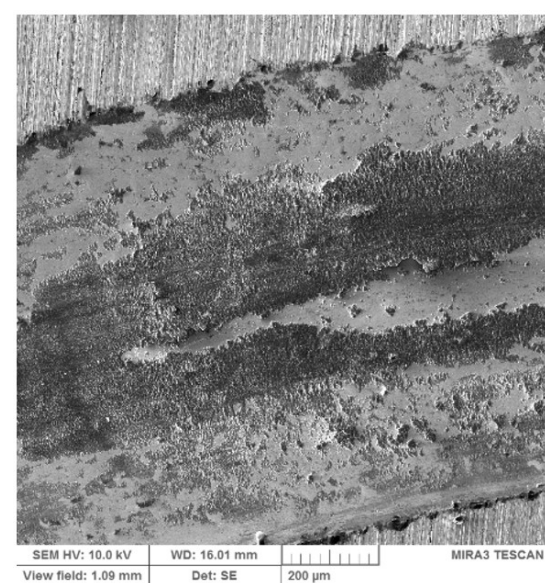

(a)

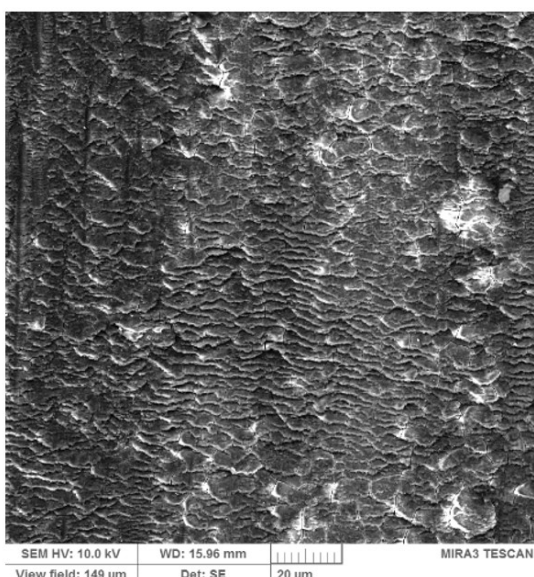

(b)

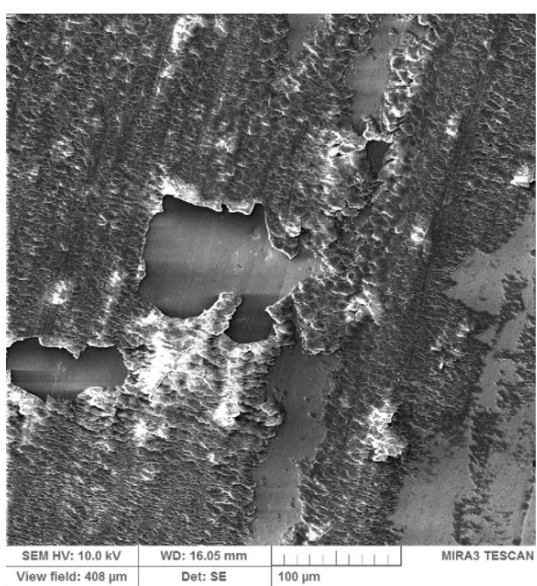

(c)

Figure 4-25: Worn surface of Stellite 21 continuously tested at $50 \mathrm{rpm}$ under $15 \mathrm{~N}$ for $20 \mathrm{hrs}$ $(1.88 \mathrm{~km})$ at (a) low magnification, (b) high magnification, (c) medium magnification.

With an increase in duration (time/distance), it can be seen from Figure 4-26 that an oxide layer has formed at certain locations within the wear track. This oxide layer appears to show cracking and some removal during the wear process (Figure 4-26b).

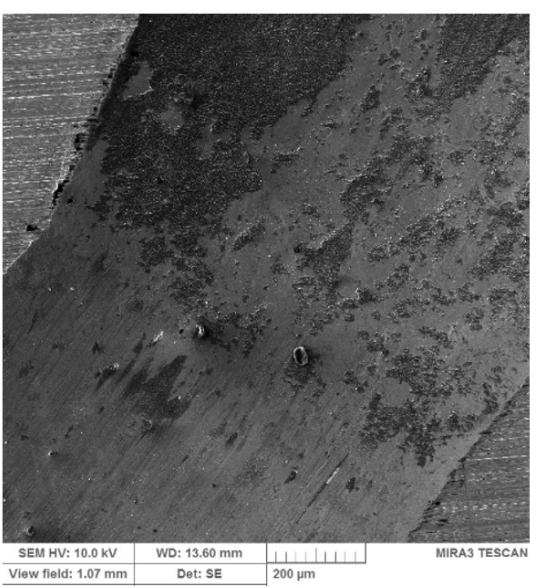

(a)

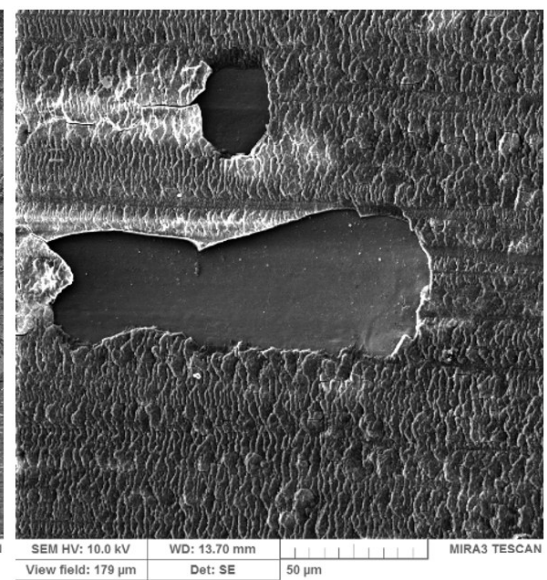

(b)

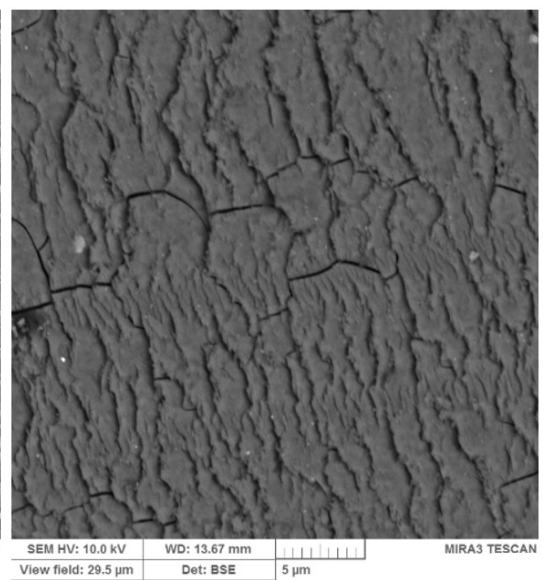

(c)

Figure 4-26: Worn surface of Stellite 21 continuously tested at $50 \mathrm{rpm}$ under $15 \mathrm{~N}$ for $40 \mathrm{hrs}$ $(3.77 \mathrm{~km})$ at (a) low magnification, (b) medium magnification, (c) high magnification. 
Under a higher normal load of $25 \mathrm{~N}$ the worn surface appears similar to the specimen tested under $15 \mathrm{~N}$ but more accentuated with higher degrees of surface deformation and scoring (Figure 4-27 and Figure 4-28).

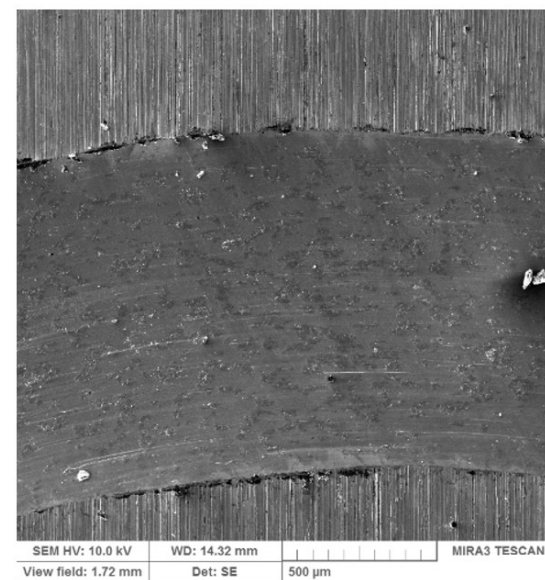

(a)

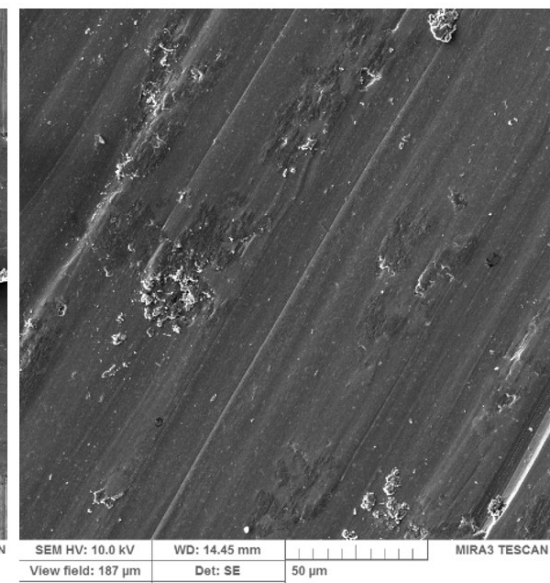

(b)

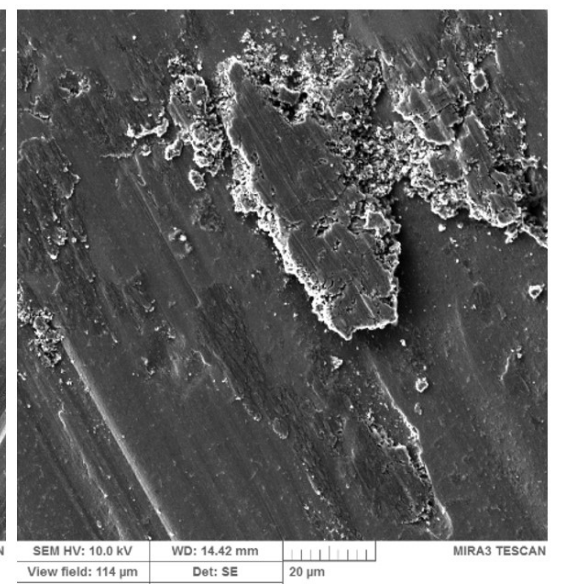

(c)

Figure 4-27: Worn surface of Stellite 21 continuously tested at $50 \mathrm{rpm}$ under $25 \mathrm{~N}$ for $10 \mathrm{hrs}$ (0.94 km) at (a) low magnification, (b) medium magnification, (c) high magnification.

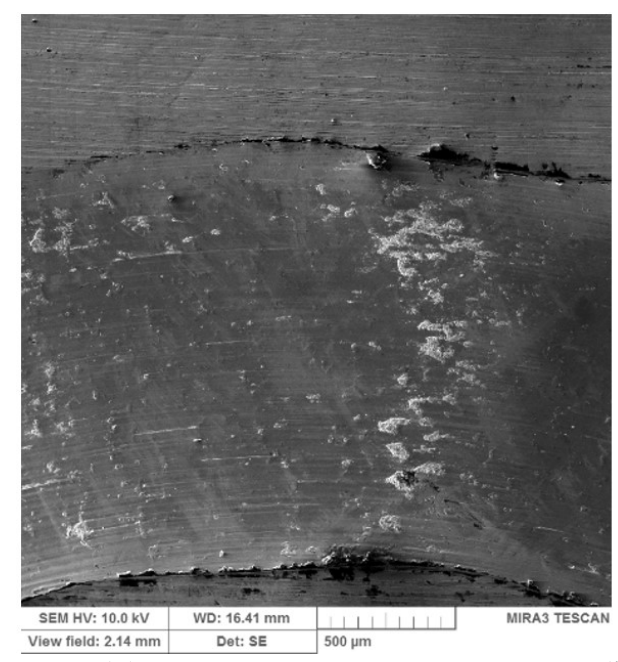

(a)

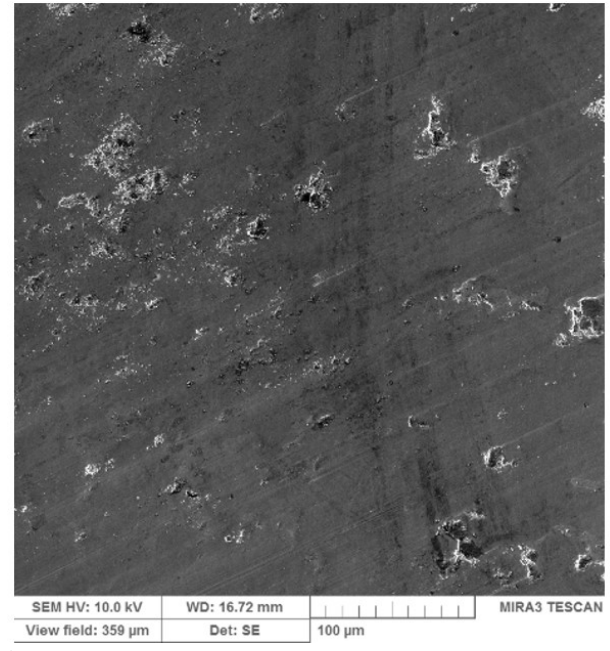

(b)

Figure 4-28: Worn surface of Stellite 21 continuously tested at $50 \mathrm{rpm}$ under $25 \mathrm{~N}$ for $20 \mathrm{hrs}$ $(1.88 \mathrm{~km})$ at (a) low magnification, (b) medium magnification. 
The feature of the wear track at the higher load of $25 \mathrm{~N}$ and duration (40 hrs) at $50 \mathrm{rpm}$ show extensive build-up in particular areas (Figure 4-29). This build up assists in forming a 'wormlike' feature around the periphery of the wear track showing preferential wear loss (Figure 4-7f).

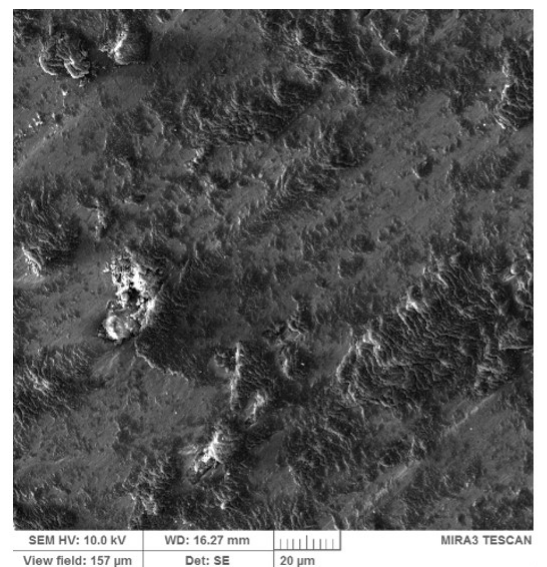

(a)

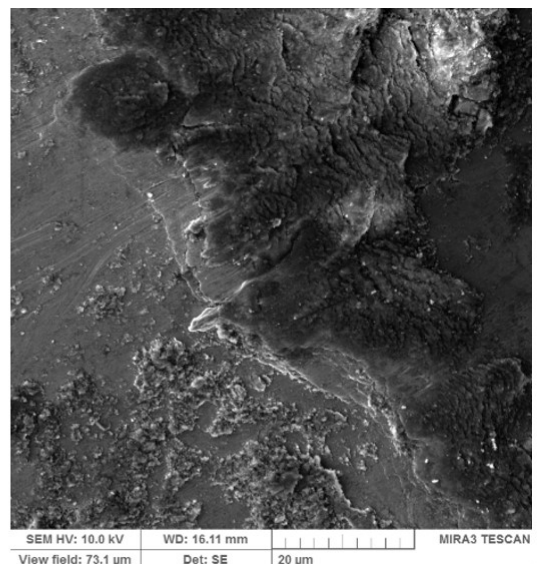

(b)

Figure 4-29: Worn surface of Stellite 21 continuously tested at $50 \mathrm{rpm}$ under $25 \mathrm{~N}$ for $40 \mathrm{hrs}$ $(3.77 \mathrm{~km})$ at (a) medium magnification, (b) high magnification.

The interrupted tests at $50 \mathrm{rpm}$ and $60 \mathrm{rpm}$ under $25 \mathrm{~N}$ showed similar features to the continuous tests, although the extent of the total damage was visually less. The worm-like features are still apparent (Figure 4-30a, and Figure 4-31a), as well as the accumulated particles from plastic deformation (Figure 4-31b). The worm-like wear track, which consists of narrowing and widening sections with also varying depth, is probably caused by a large amount of oxidized debris buildups being pushed through the track. The WC ball then moved in effect like an almost up-and-down roller coaster to overcome the hard oxide build-up, which made the preferential wear track topography. 


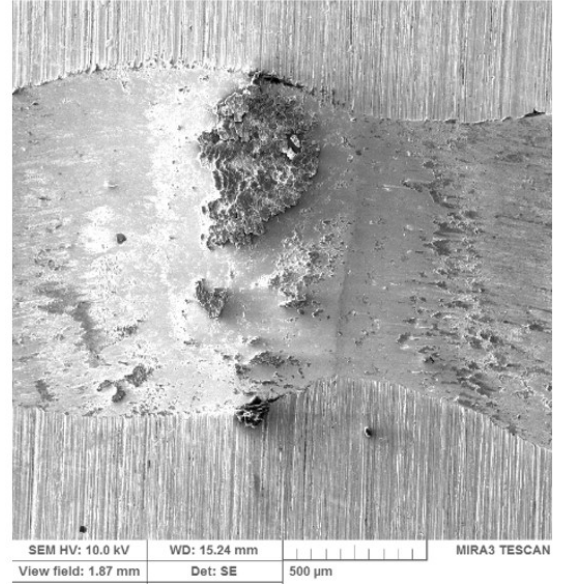

(a)

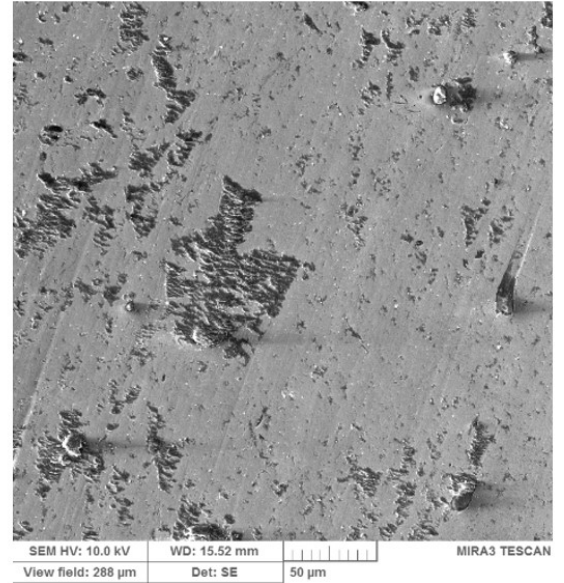

(b)

Figure 4-30: Worn surface of Stellite 21 after interrupted test at $50 \mathrm{rpm}$ under $25 \mathrm{~N}$ for $40 \mathrm{hrs}$ $(3.77 \mathrm{~km})$ at (a) low magnification, (b) medium magnification.

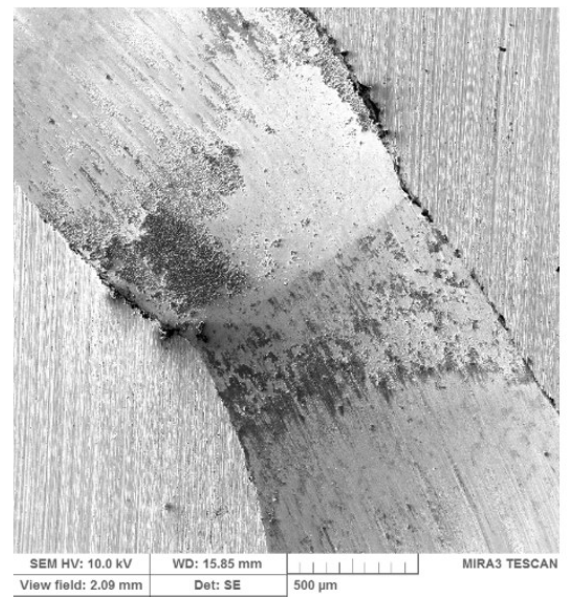

(a)

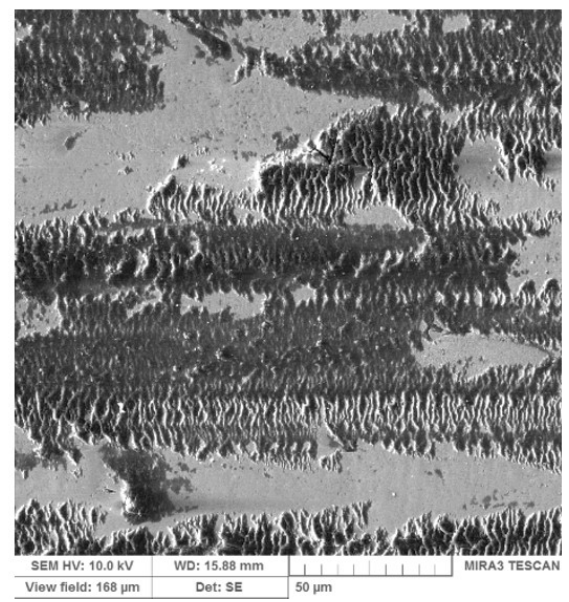

(b)

Figure 4-31: Worn surface of Stellite 21 after interrupted test at $60 \mathrm{rpm}$ under $25 \mathrm{~N}$ for $40 \mathrm{hrs}$ $(3.77 \mathrm{~km})$ at (a) low magnification, (b) medium magnification.

In summary, the wear tracks from testing of Stellite 21 show mostly ploughing and scoring with debris building up along the wear track. This stems from the low volume fraction of carbides and consequent removal of softer solid-solution. An oxide layer can be identified which subsequently cracks and breaks down in the wear track. 


\subsubsection{Worn Surfaces of Stellite 720}

Stellite 720 experienced the least amount of volume loss during the continuous and interrupted tests, and did not show significant ploughing and scoring of the surface such as that identified in Stellite 21. As shown in Figure 4-32, scoring and oxide build up is evident at $15 \mathrm{~N}$ for a short duration of 10 hrs. Staircasing is evident at $15 \mathrm{~N}$ for 20 hrs as shown in Figure 4-33, and is more pronounced as the duration continues up to 20 hrs (Figure 4-34). A particular instance of staircasing can be identified adjacent to a large blocky carbide (Figure 4-34b). This indicates that the particles were running into a hard phase and stopping at this interface where a slight difference in height had developed from wearing away of the softer surrounding matrix. An oxide layer with staricasing features can be identified in the wear track (Figure 4-32c). This oxidized surface layer shows extensive cracking and breaking even under $15 \mathrm{~N}$ for $10 \mathrm{hrs}$, and Figure 4-32c shows a single instance of a particle breaking away from the surface, which would have then been pulled into the wear track. This network of cracks in the oxide and the onslaught of the WC ball will eventually cause more particles to break free and be engulfed into the wear tack. The tenacity of this oxide layer and the subsequent properties of the Stellite will ultimately result in how the wear process continues. 


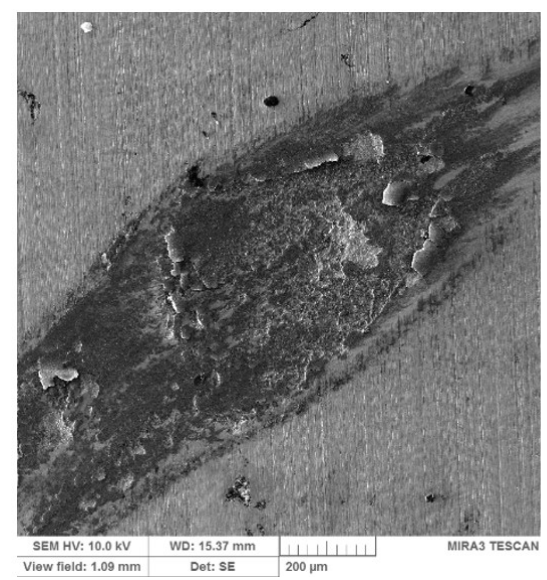

(a)

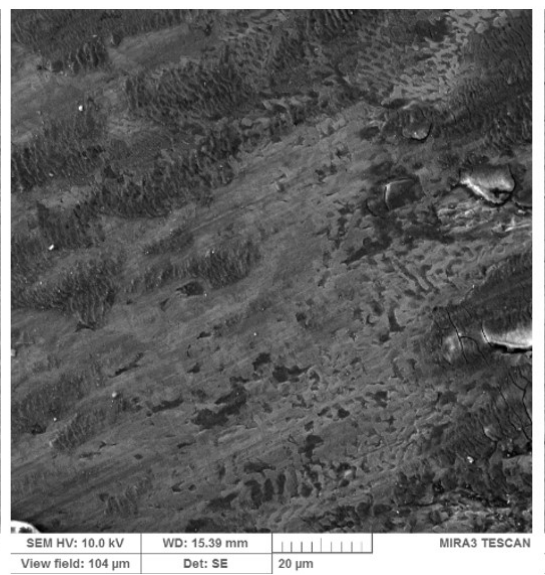

(b)

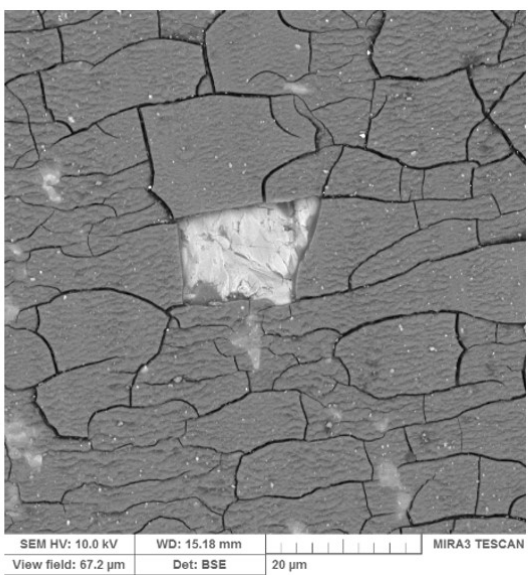

(c)

Figure 4-32: Worn surface of Stellite 720 continuously tested at $50 \mathrm{rpm}$ under $15 \mathrm{~N}$ for $10 \mathrm{hrs}$ $(0.94 \mathrm{~km})$ at (a) low magnification, (b) medium magnification, (c) high magnification.

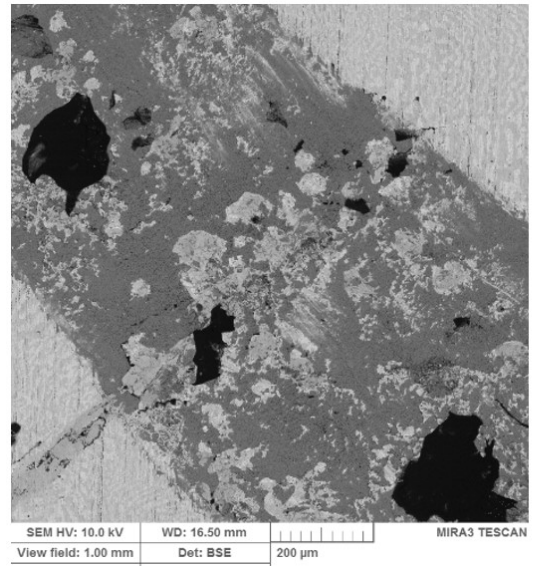

(a)

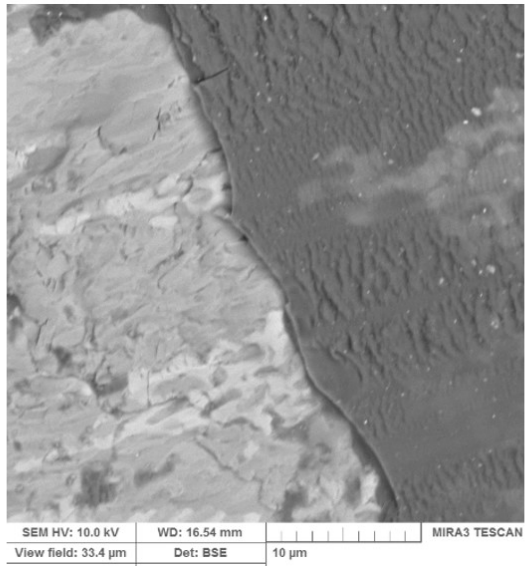

(b)

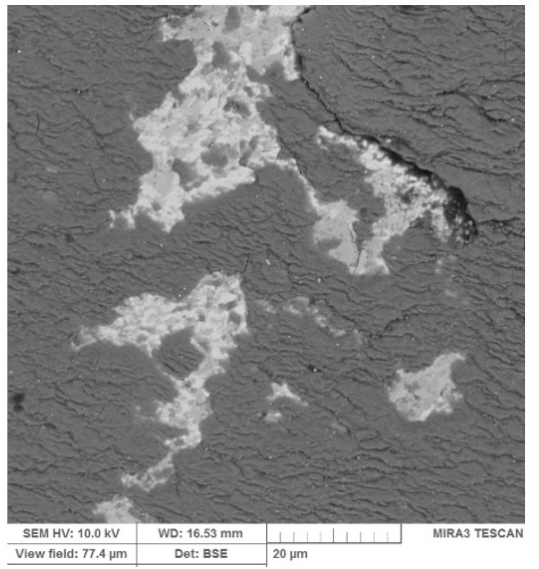

(c)

Figure 4-33: Worn surface of Stellite 720 continuously tested at $50 \mathrm{rpm}$ under $15 \mathrm{~N}$ for $20 \mathrm{hrs}$ $(1.88 \mathrm{~km})$ at (a) low magnification, (b) high magnification, (c) medium magnification. 


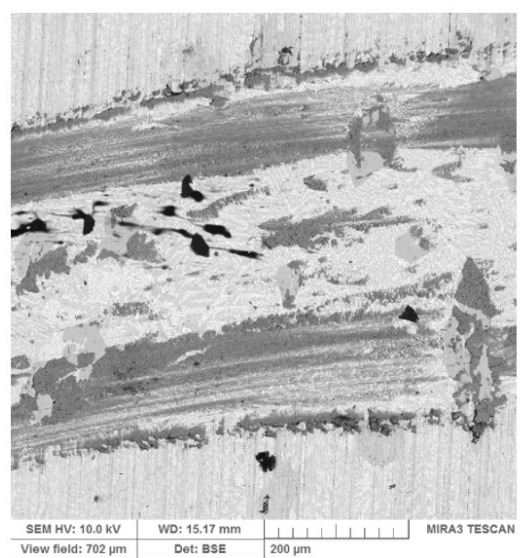

(a)

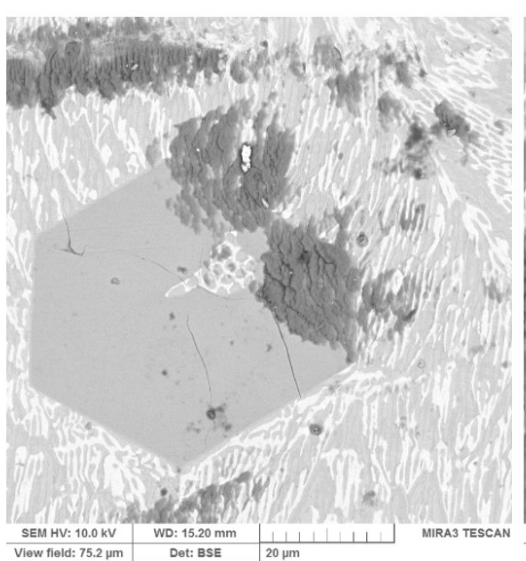

(b)

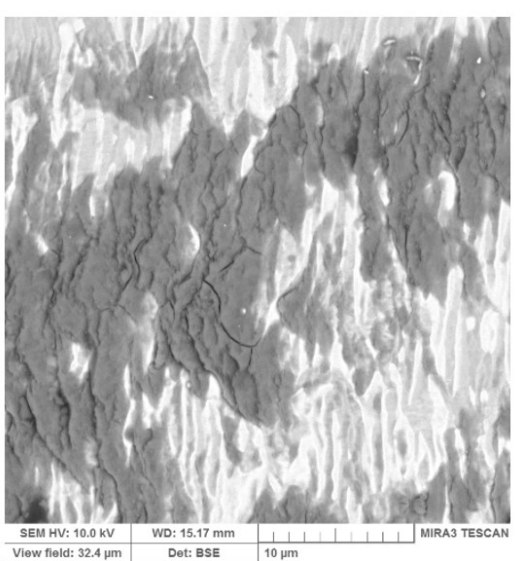

(c)

Figure 4-34: Worn surface of Stellite 720 continuously tested at $50 \mathrm{rpm}$ under $15 \mathrm{~N}$ for $40 \mathrm{hrs}$ $(3.77 \mathrm{~km}$ ) at (a) low magnification, (b) medium magnification, (c) high magnification.

A greater degree of surface oxide can be identified on the specimen tested at $25 \mathrm{~N}$ loading (Figure 4-35). This is further accentuated at longer durations of $20 \mathrm{hrs}$ and 40 hours (Figure 4-36 and Figure 4-37). In some cases, large oxide particles have formed on the surface (Figure 4-35a). A fine network of cracks in the oxide layer due to the loading can also be identified, as seen in Figure 4-36b. Heavy staircasing can be identified in Figure 4-37b at $40 \mathrm{hrs}$ duration where it would be expected to see more wear after the oxide has been broken down. 


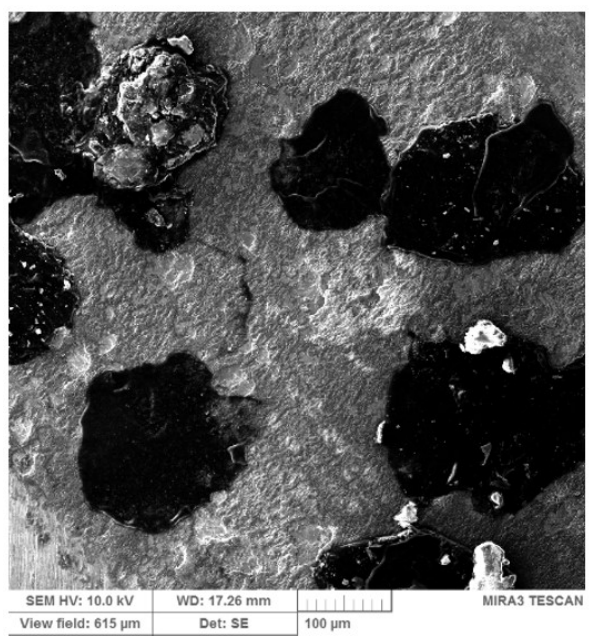

(a)

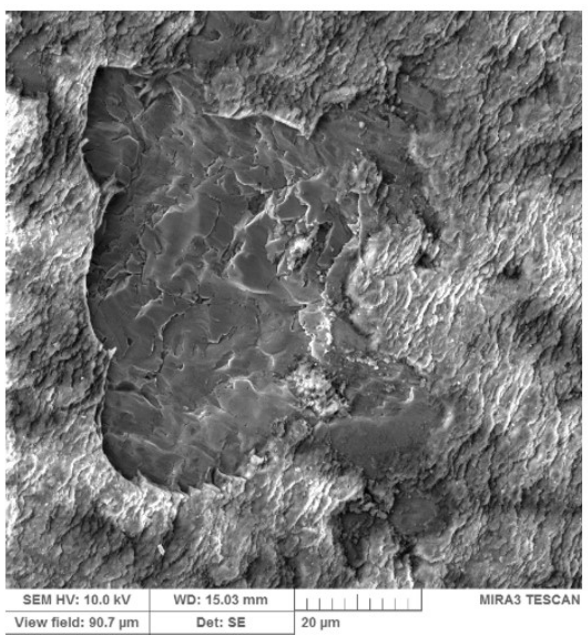

(b)

Figure 4-35: Worn surface of Stellite 720 continuously tested at $50 \mathrm{rpm}$ under $25 \mathrm{~N}$ for $10 \mathrm{hrs}$ $(0.94 \mathrm{~km})$ at (a) low magnification, (b) medium magnification.

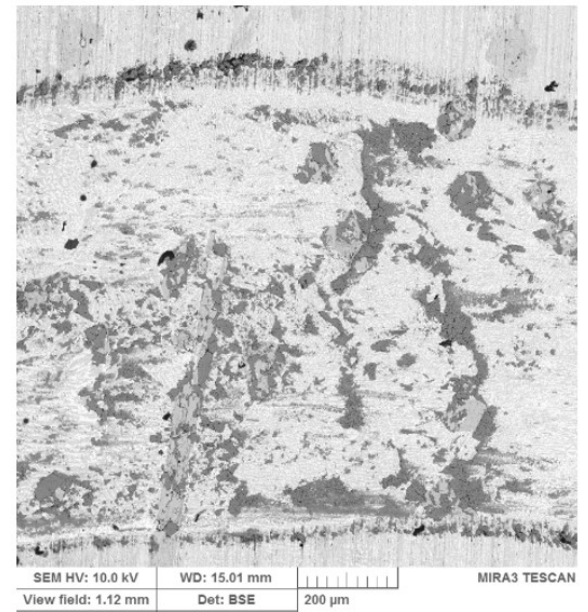

(a)

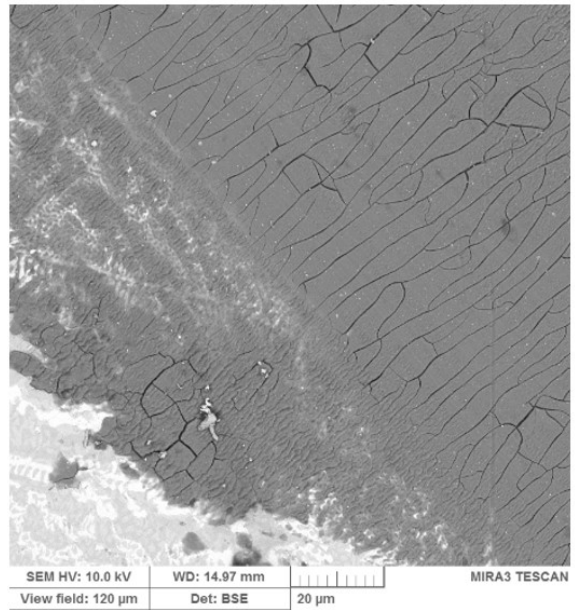

(b)

Figure 4-36: Worn surface of Stellite 720 continuously tested at $50 \mathrm{rpm}$ under $25 \mathrm{~N}$ for $20 \mathrm{hrs}$ $(1.88 \mathrm{~km})$ at (a) low magnification, (b) medium magnification. 


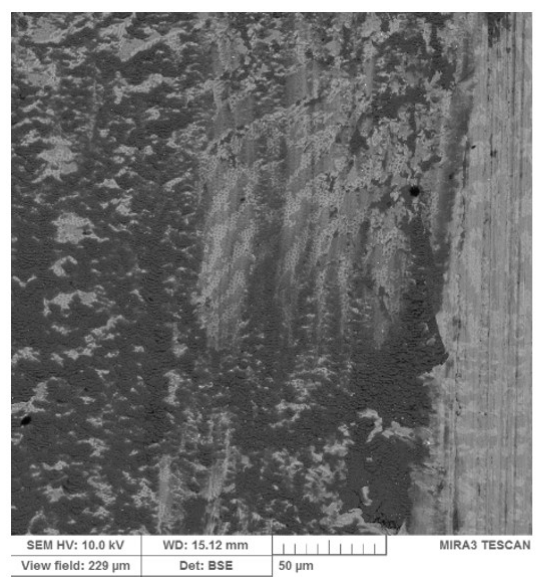

(a)

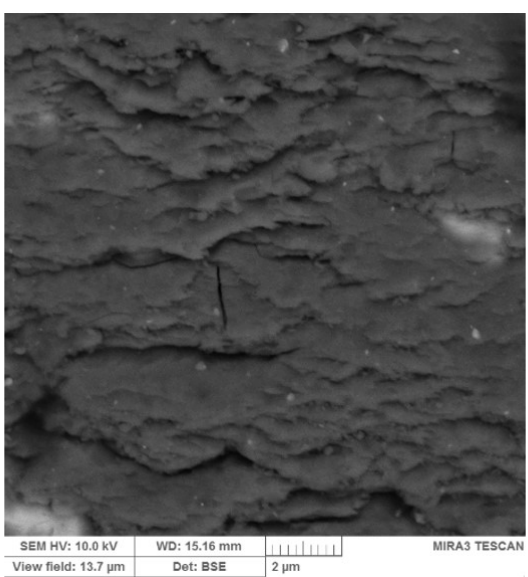

(b)

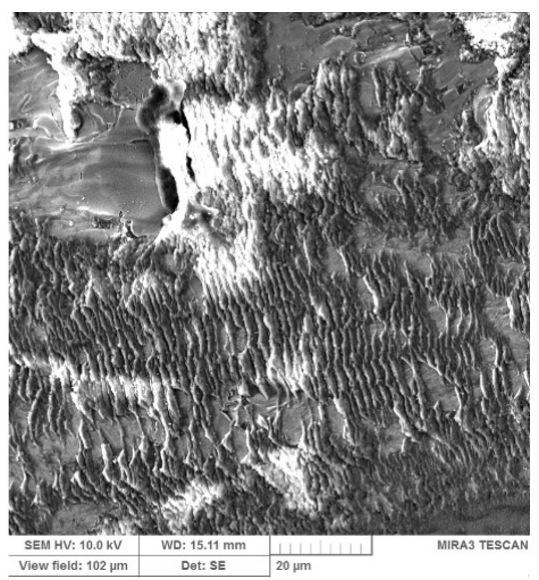

(c)

Figure 4-37: Worn surface of Stellite 720 continuously tested at $50 \mathrm{rpm}$ under $25 \mathrm{~N}$ for $40 \mathrm{hrs}$ $(3.77 \mathrm{~km})$ at (a) low magnification, (b) high magnification, (c) medium magnification.

The interrupted tests at $50 \mathrm{rpm}$ and $60 \mathrm{rpm}$ under $25 \mathrm{~N}$ showed similar features. Scoring of the surface oxide layer is evident in Figure 4-38 where particular areas appear to have built up and then been worn away. This could also indicate that preferential staircasing has occurred at these particular sites along the wear track. Stellite 720 contains a large block Cr-rich carbide with a hexagonal shape, which did show to be a site for preferential build-up in some localized areas (Figure 38a - large grey particle, centre image). During the analysis of the wear track, cracking and spalling of the large carbide particle can be identified, as shown in Figure 4-39b. The spalling and breaking of the carbides will introduce very hard particles into the wear track, which should in theory increase wear. 


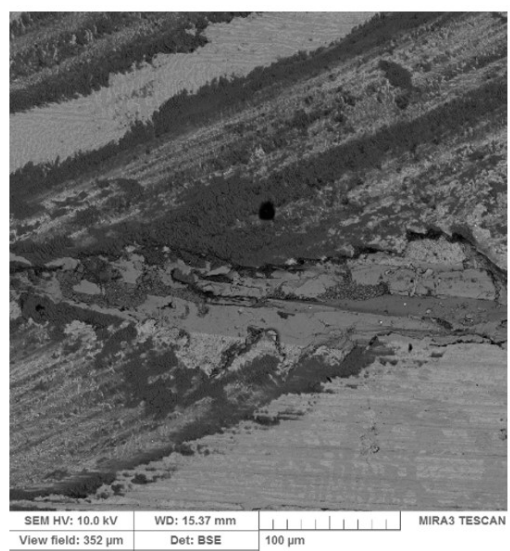

(a)

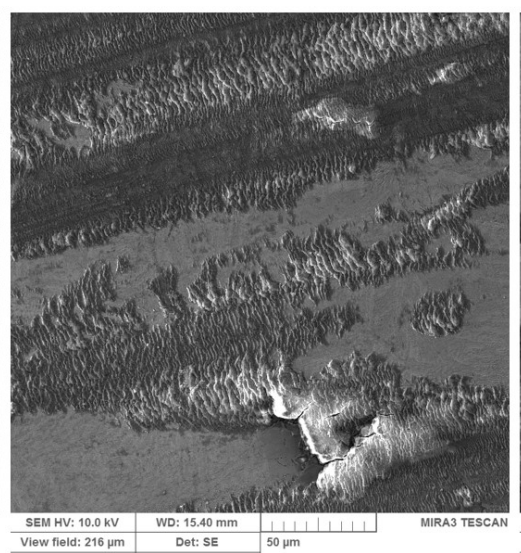

(b)

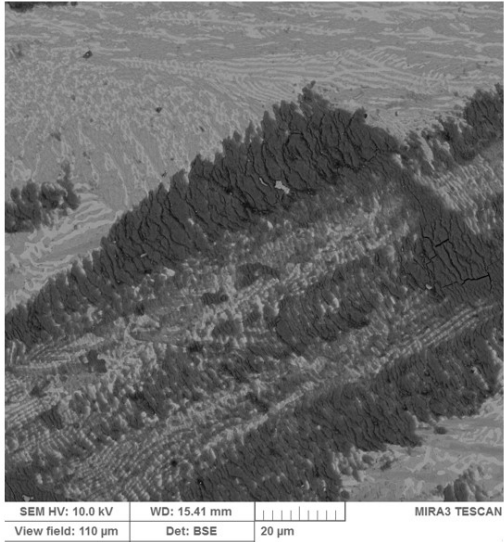

(c)

Figure 4-38: Worn surface of Stellite 720 after interrupted test at $50 \mathrm{rpm}$ under $25 \mathrm{~N}$ for $40 \mathrm{hrs}$ $(3.77 \mathrm{~km})$ at (a) low magnification, (b) medium magnification, (c) high magnification.

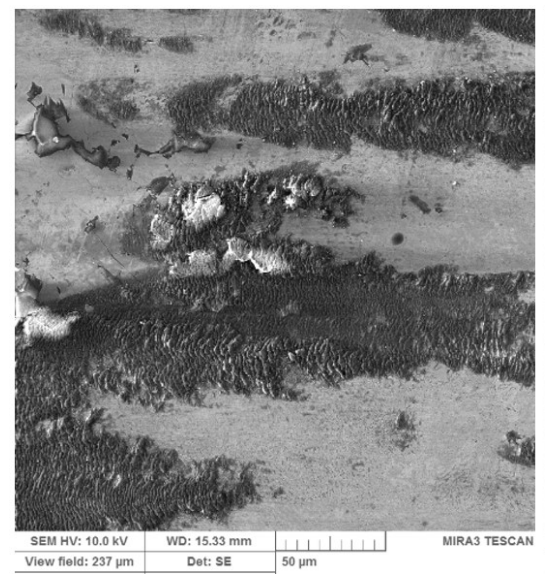

(a)

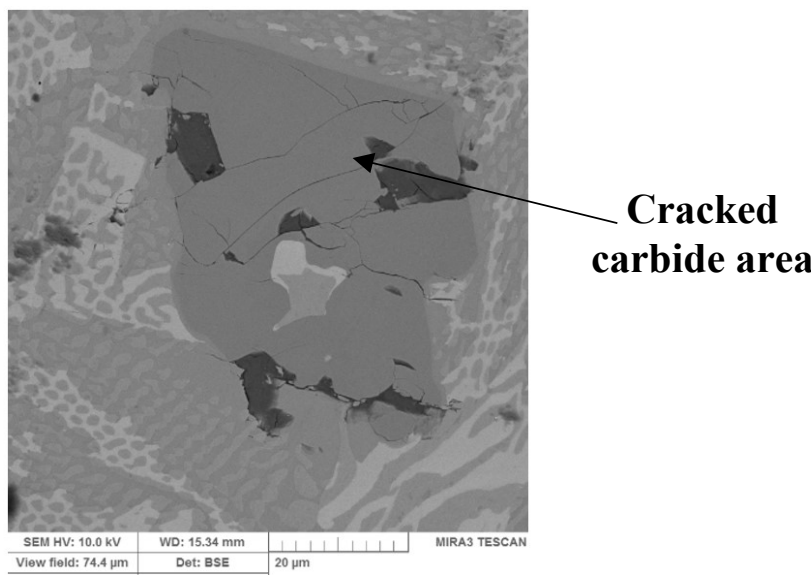

(b)

Figure 4-39: Worn surface of Stellite 720 after interrupted test at $60 \mathrm{rpm}$ under $25 \mathrm{~N}$ for $40 \mathrm{hrs}$ $(3.77 \mathrm{~km})$ at (a) low magnification, (b) high magnification.

In summary, Stellite 720 appears to show a significant amount of oxide layer driven features. Very little ploughing and scoring of the surface could be identified. Instead, a build-up of oxidized particles on the surface appears to be a feature of this alloy. A fine network of cracks developed in the oxide layer and some particles spalled off into the wear path. In general, Stellite 720 still showed a consistently low volume loss during all tests, which indicates that the oxide 
layer is relatively tough in terms of its adherence to the surface. In addition, very little fracture of the large Cr-rich carbides could be identified in the analysis. The volume loss coupled with the relatively tough oxide layer indicates that the size and distribution of carbides in this alloy are favourable for sliding wear at these loads and speeds.

\subsubsection{Worn Surfaces of Stellite 728}

Stellite 728 appears to show traits of both Stellite 21 and Stellite 720. The wear track shows worm-like features around the periphery (Figure 4-44a), also ploughing and scoring of the surface (Figure 4-40b and Figure 4-40c). This is indicative of plastic deformation of the solid solution matrix material. Figure 4-41 shows scoring of the oxide layer as shown in Stellite 720 as the duration increases. As the duration is increased further, Figure 4-42 shows wear debris being deposited in the vicinity of the carbides. Particularly in Figure 4-42a where appears to form adjacent to the carbides, which is similar to that seen for the Cr-rich carbides in Stellite 720. The volume loss overall for the Stellite 728 samples is slightly higher than Stellite 720, which indicates that the carbides play a major role in preventing wear loss under these particular conditions. 


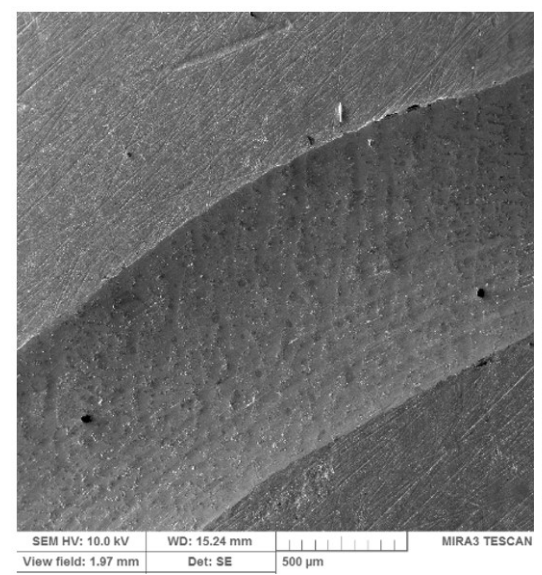

(a)

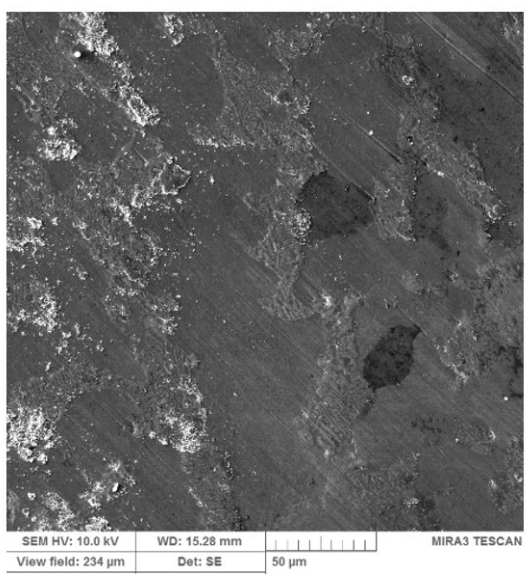

(b)

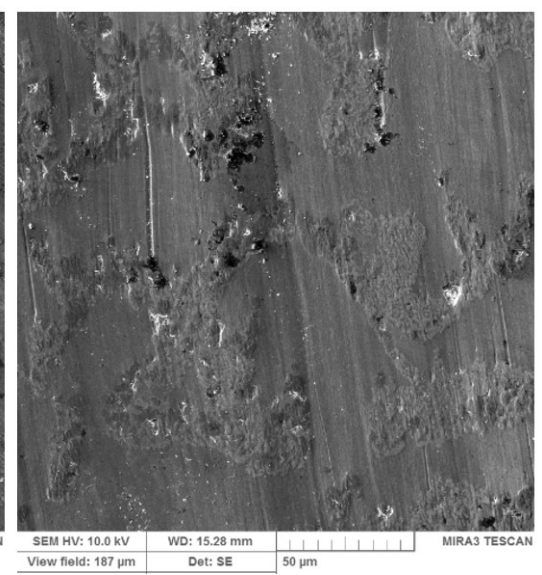

(c)

Figure 4-40: Worn surface of Stellite 728 continuously tested at $50 \mathrm{rpm}$ under $15 \mathrm{~N}$ for $10 \mathrm{hrs}$ (0.94 km) at (a) low magnification, (b) medium magnification, (c) high magnification.

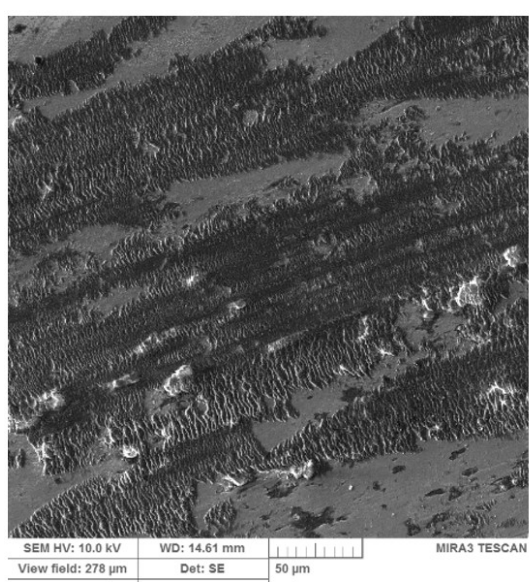

(a)

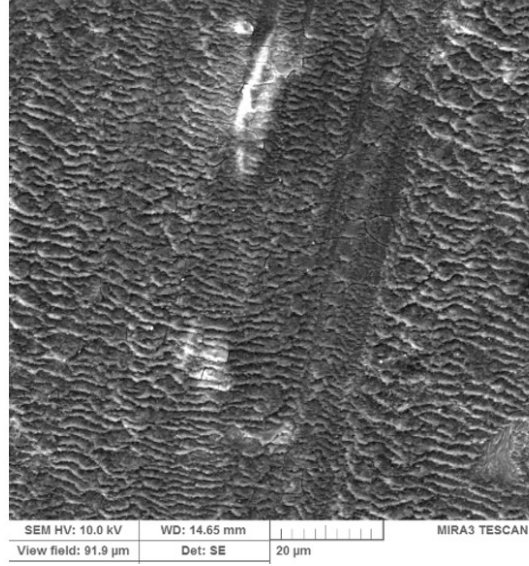

(b)

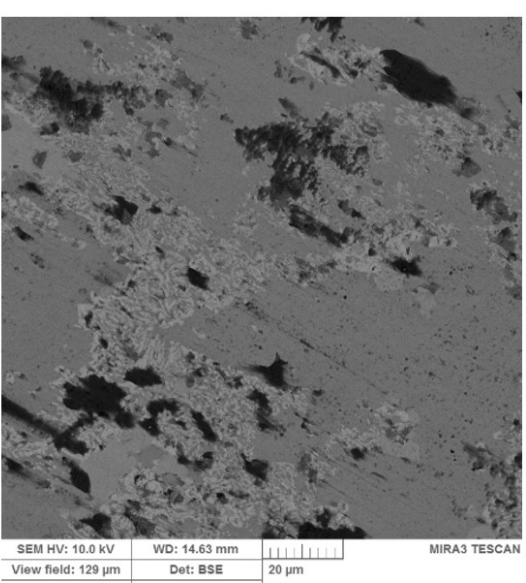

(c)

Figure 4-41: Worn surface of Stellite 728 continuously tested at $50 \mathrm{rpm}$ under $15 \mathrm{~N}$ for $20 \mathrm{hrs}$ $(1.88 \mathrm{~km})$ at (a) low magnification, (b) high magnification, (c) medium magnification. 


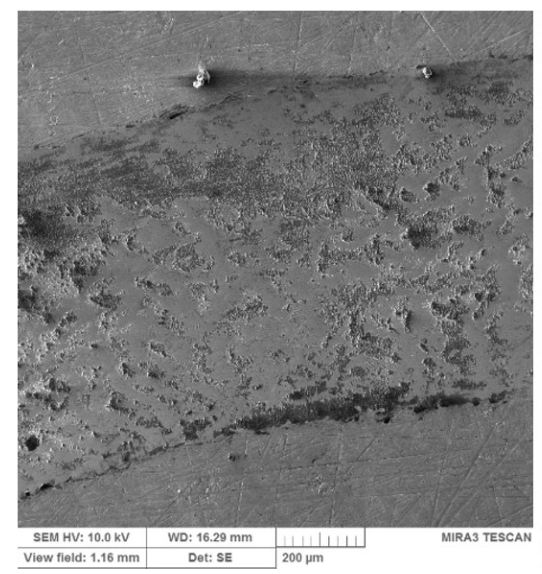

(a)

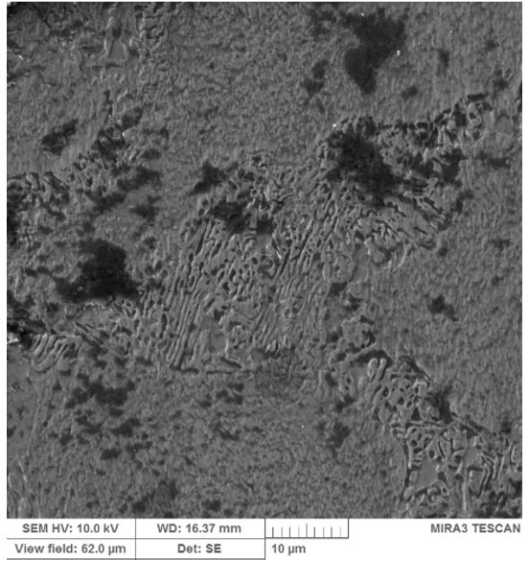

(b)

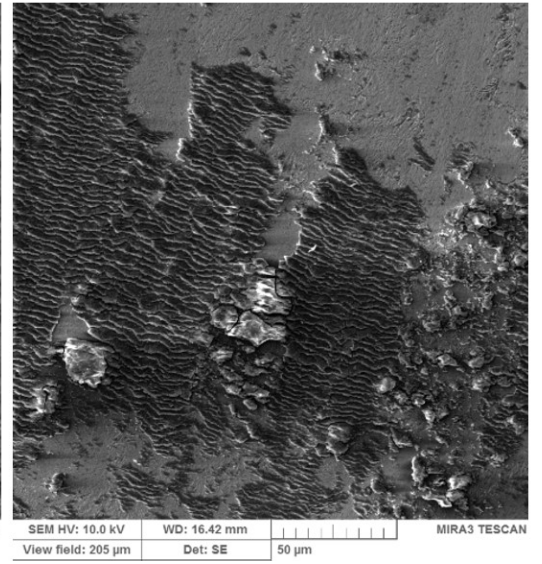

(c)

Figure 4-42: Worn surface of Stellite 728 continuously tested at $50 \mathrm{rpm}$ under $15 \mathrm{~N}$ for 40 hrs (3.77 km) at (a) low magnification, (b) high magnification, (c) medium magnification.

Figure 4-43 shows both a BSE and a SE image of the same area side by side. These images show the difference in performing an evaluation using BSEs or SEs. The SE image (Figure 4-43b) shows the wear debris 'charging' and sitting on the surface. In this image it is not obvious if any pattern or any data can be concluded. When the BSE image (Figure 4-43a) is analyzed, the bulk of the wear debris appears to sit at the dendritic carbide (white areas) and solid solution (light grey) interfaces. This shows how the carbides are acting similar to the blocky carbides in Stellite 720 . 


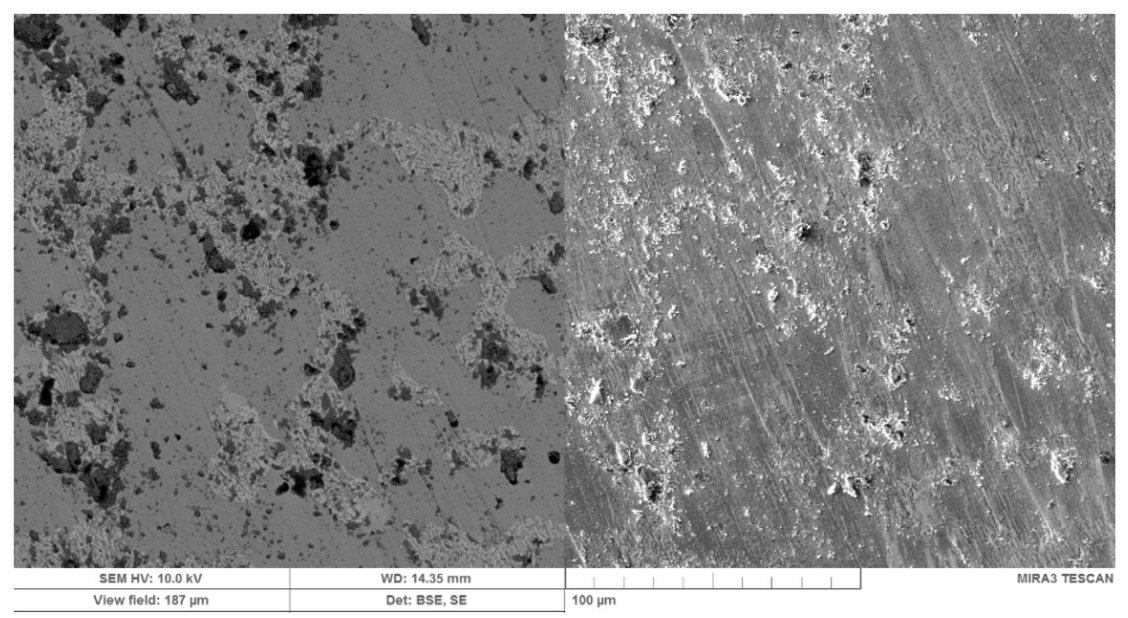

(a)

(b)

Figure 4-43: Worn surface of Stellite 728 continuously tested at $50 \mathrm{rpm}$ under $25 \mathrm{~N}$ for $10 \mathrm{hrs}$ $(0.94 \mathrm{~km})$ (a) BSE image of a local area, (b) SE image of the same location.

A worm-like profile around the periphery of the wear track can be identified in Figure 44a. In addition, staircasing and a build-up of oxide debris are also visible on the wear tracks of the specimen tested for a longer duration (Figure 4-44b and Figure 4-44 respectively). This shows that Stellite 728 is acting similar to Stellite 21 in that a worm-like profile is being established with a build-up of debris. However, at the same time, staircasing and cracking of the oxide layer is also occurring which is similar to the features found the Stellite 720 wear tracks. 


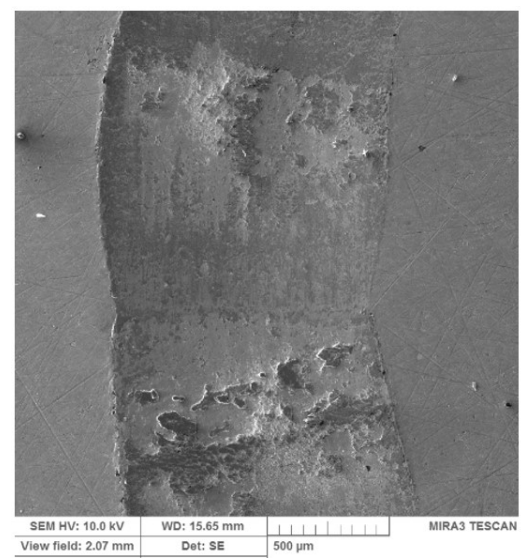

(a)

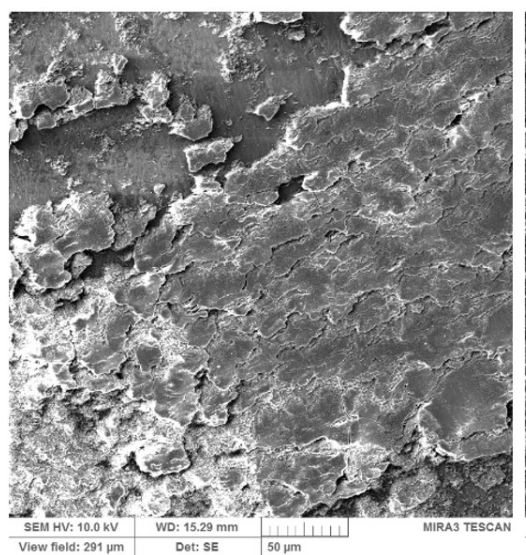

(b)

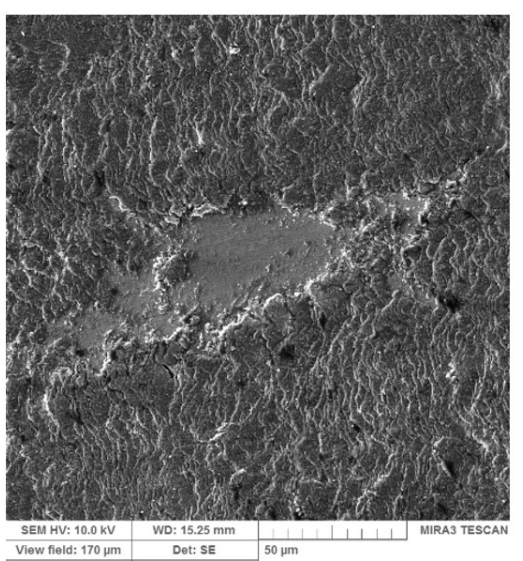

(c)

Figure 4-44: Worn surface of Stellite 728 continuously tested at $50 \mathrm{rpm}$ under $25 \mathrm{~N}$ for $20 \mathrm{hrs}$ (1.88 km). (a) low magnification, (b) medium magnification, (c) high magnification.

The longest duration at $40 \mathrm{hrs}$ under $25 \mathrm{~N}$ shows the staircasing profile of debris build-up (Figure 4-45a) as well as preferential deposits of debris in the carbide zones (Figure 4-45b). This is an interesting feature and could be explained by the removal of solid solution from between the carbides leaving 'holes' in localized areas. Debris could easily be anchored to the small lamellar carbides and show preferential deposits in these areas.

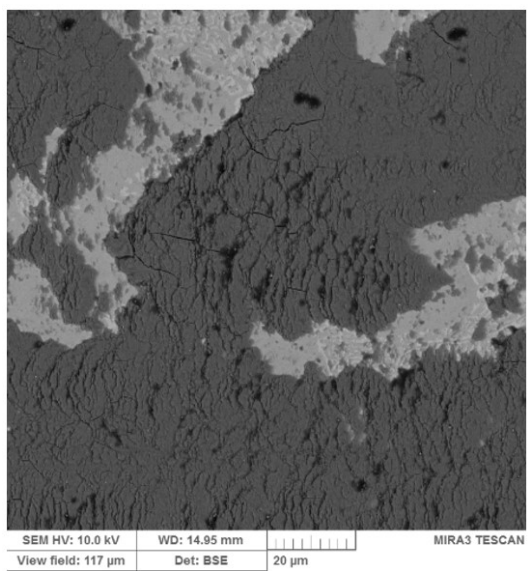

(a)

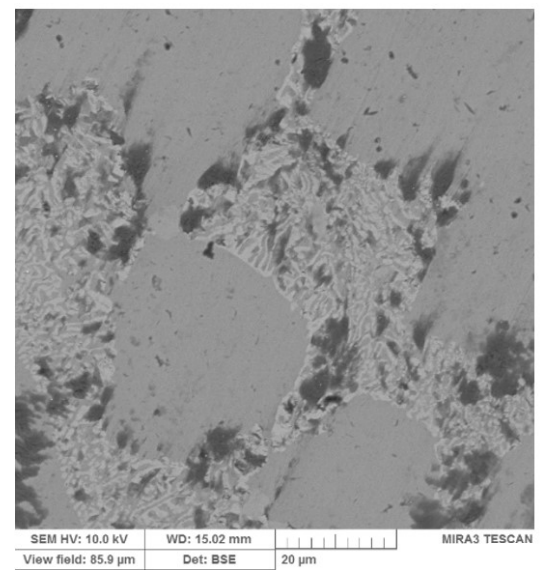

(b)

Figure 4-45: Worn surface of Stellite 728 continuously tested at $50 \mathrm{rpm}$ under $25 \mathrm{~N}$ for $40 \mathrm{hrs}$ $(3.77 \mathrm{~km})$ at (a) medium magnification, (b) high magnification. 
The interrupted tests showed an interesting feature of wear debris bring aligned with the carbide formation as shown in Figure 4-46a and 4-47b. The carbides in Stellite 728 are high in niobium which may form particularly hard carbides to combat wear, which could be considered a wear feature of the alloy. In addition, a network of fine cracks in the oxide layer such as that seen in Stellite 720 can also be identified (Figure 4-46c).

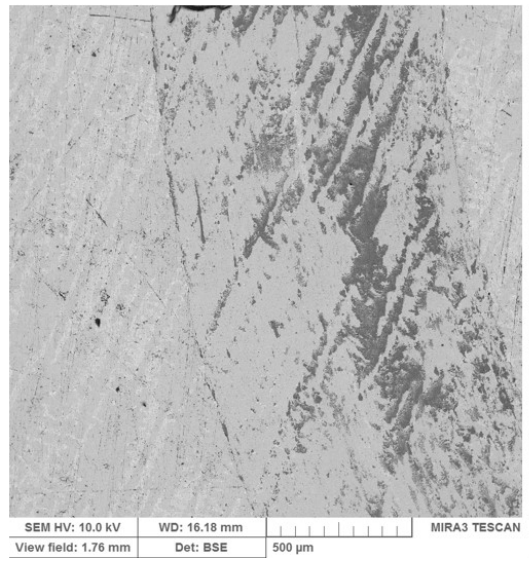

(a)

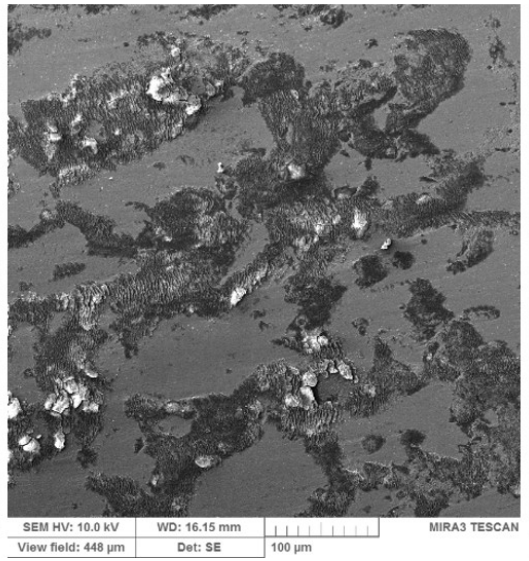

(b)

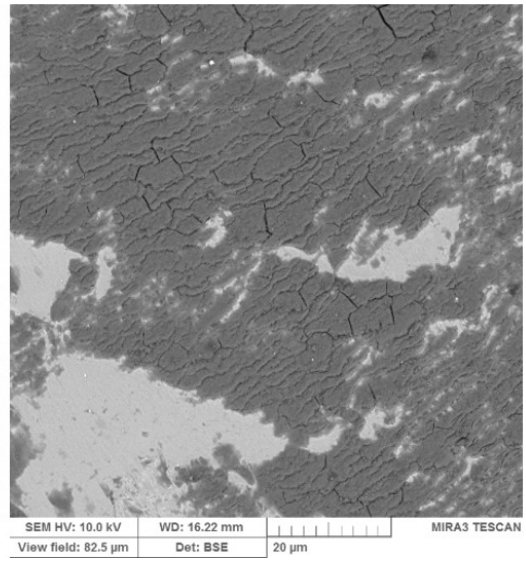

(c)

Figure 4-46: Worn surface of Stellite 728 after interrupted test at $50 \mathrm{rpm}$ under $25 \mathrm{~N}$ for 40 hrs $(3.77 \mathrm{~km})$ at (a) low magnification, (b) medium magnification, (c) high magnification.

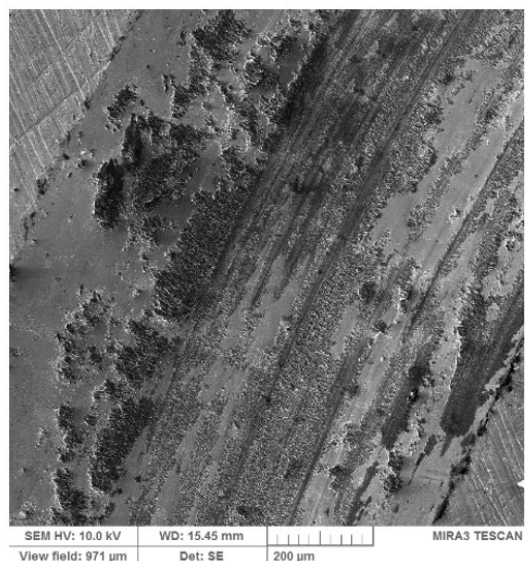

(a)

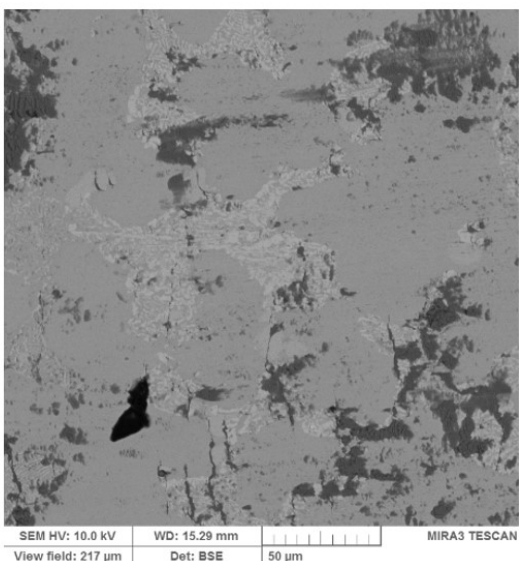

(b)

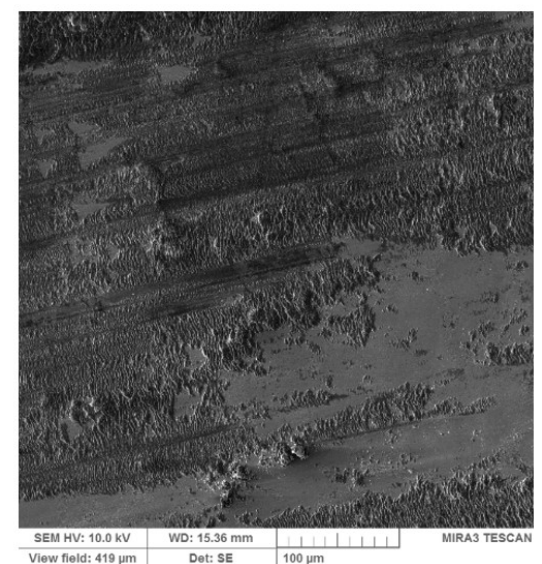

(c)

Figure 4-47: Worn surface of Stellite 728 after interrupted test at $60 \mathrm{rpm}$ under $25 \mathrm{~N}$ for 40 hrs (3.77 km) at (a) low magnification, (b) medium magnification, (c) low magnification. 
A feature which is not particularly prevalent in the analysis is the cracking of the carbide structure. An example of cracking in the carbide structure is shown in Figure 4-48. This would suggest that the fine nature of the carbides in Stellite 728 are quite tough and rigidly hold their position in the microstructure.

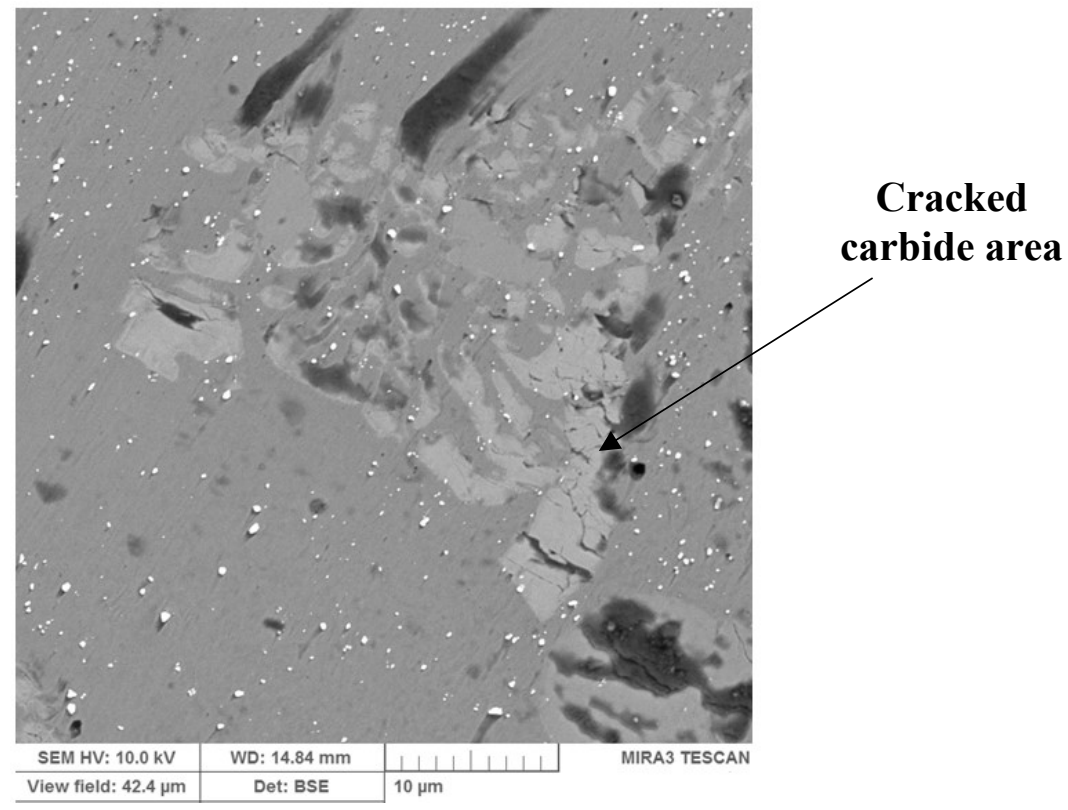

Figure 4-48: Cracking in carbide structure of Stellite 728 worn surface.

In summary, Stellite 728 appears to show features of both Stellite 21 and Stellite 720 . There was an initial ploughing and scoring mechanism occurring at the lower loading and shorter durations which exposed the carbides via removal of the solid solution in the adjacent areas. The carbides stood on the surface shortly and then became obstacles for the WC ball. However, as the duration was extended an extensive staircasing profile of oxidized particles began to develop. The staircasing in Stellite 728 surface is more pronounced than that seen on Stellite 720 surface, which indicates a slightly 'softer' oxide forming on the surface. In addition, the build-up occurred, as like that seen in Stellite 720, adjacent to the carbides. This is an interesting feature of Stellite 728 which 
suggests that there may be fewer and smaller carbides in Stellite 728 than in Stellite 720, but their hardness and morphology are ideal for the sliding wear conditions tested in this study.

\subsection{Wear Debris Analysis}

Examination of the wear debris can also provide useful information in addition to analysis of the wear surface. The main drawback is that the wear debris may not be in the same state as when it first appeared in the wear zone. The breaking and then tracking of the wear debris as wear continues can crush and amalgamate the particles in repeated cycles prior to detachment from the surfaces.

\subsubsection{Caveats to SEM/EDX Data}

SEM/EDX analysis was performed on the wear debris generated during the interrupted testing at $60 \mathrm{rpm}$. An attempt was made to ascertain the chemical composition of the developing debris during the wear process. As previously mentioned, quantitative analysis is not generally considered easy to accomplish using SEM/EDX. Certain caveats should be considered in the approach to understanding the data [33]:

i) The electron beam is assumed to hit a volume of material that is homogeneous.

ii) The specimen must be flat and highly polished and placed at known angles to the x-ray spectrometer and incident beam. The roughness of the surface, for example, influences the interactions of electrons and the propagation of $\mathrm{x}$-rays.

iii) Chemical polishing or etching will selectively leach the constituents that the $\mathrm{x}$-ray microanalysis characterizes. 
These caveats form an important role in understanding the limitations of the SEM/EDX data. The wear debris will likely be inhomogeneous particles forming from the surface wear and oxidation processes happening in the wear track. In addition, the error whilst reading the elements on a rough surface such as the debris particles will be compounded by oxygen being a prime contributor to the analysis through oxidation. Analysis of oxygen and carbon is very difficult to evaluate using SEM/EDX but they should be included as prime elements to check for.

After taking these caveats into consideration, a direct comparison between this data set may be of some benefit to understanding the wear mechanism, and will be presented in this section.

\subsubsection{SEM Morphology of Wear Debris}

The analysis of the debris showed interesting particle morphologies occurring in the wear track. A general overview showed all three alloys having a fine distribution of wear particles in the range from $1 \mu \mathrm{m}$ or less to as larger as $10 \mu \mathrm{m}$ plus angular pieces in the debris collected (Figure 4-49 to Figure 4-52). The size population of the debris is discussed later in this chapter.

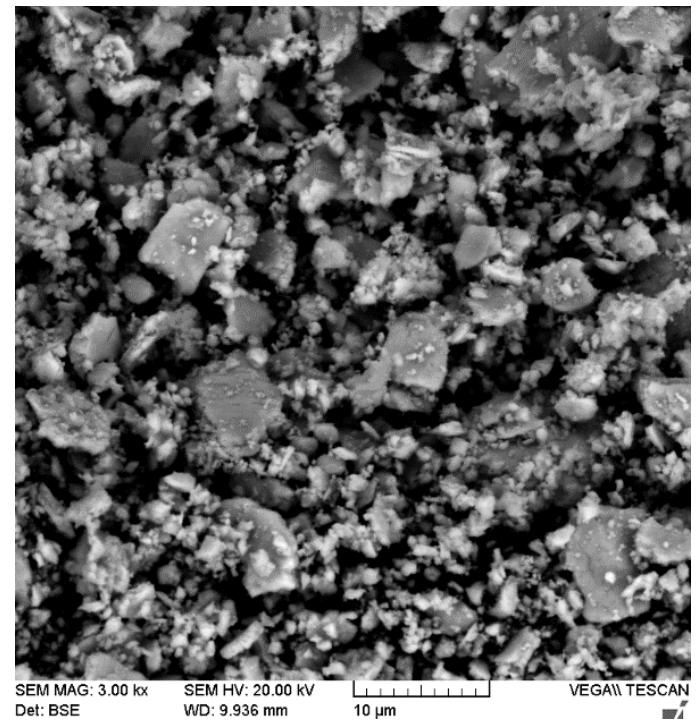

(a)

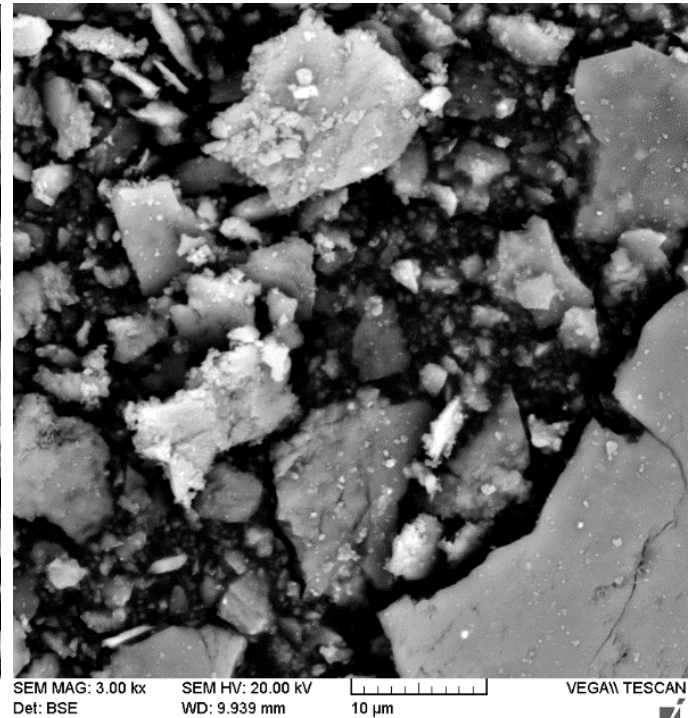

(b)

Figure 4-49: Stellite 21 wear debris at $25 \mathrm{~N} 60 \mathrm{rpm}$ for (a) 1 hour, (b) 60 hours duration. 


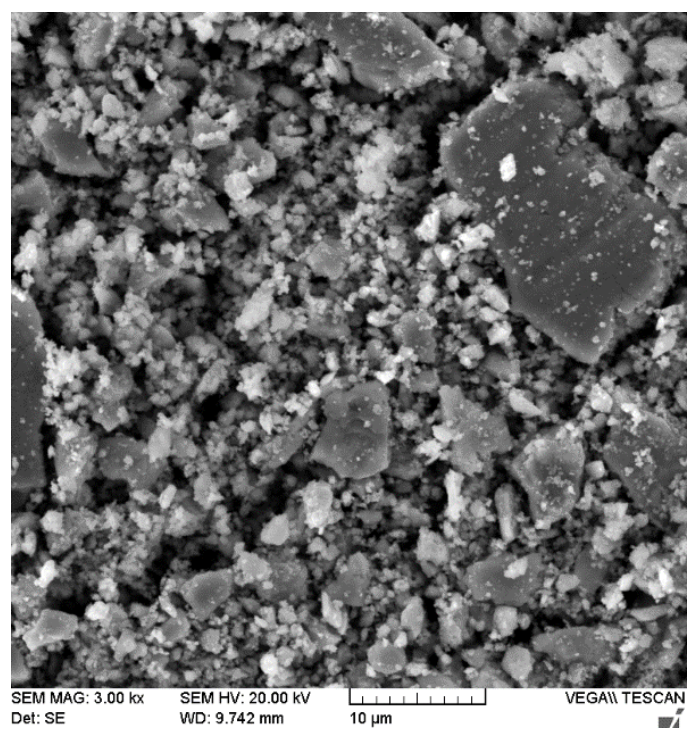

(a)

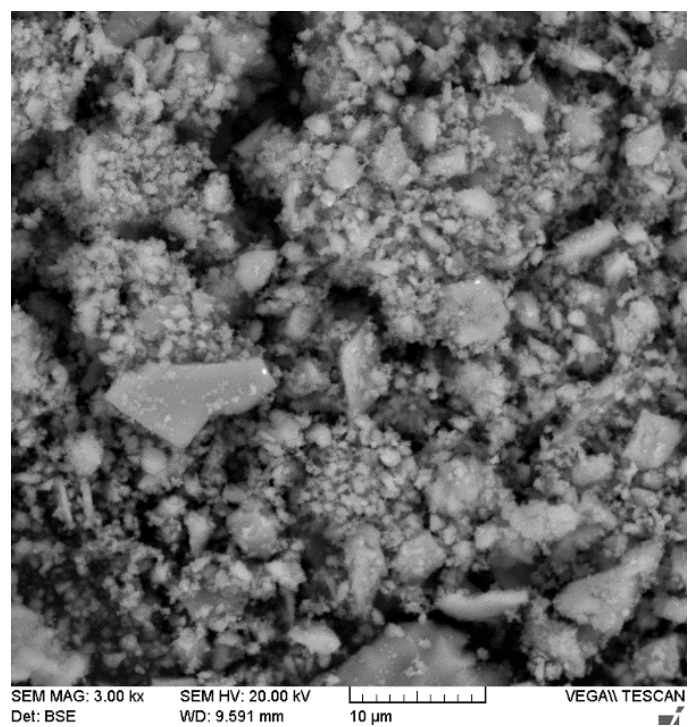

(b)

Figure 4-50: Stellite 720 wear debris at 25 N 60 rpm for (a) 1 hour, (b) 60 hours duration.

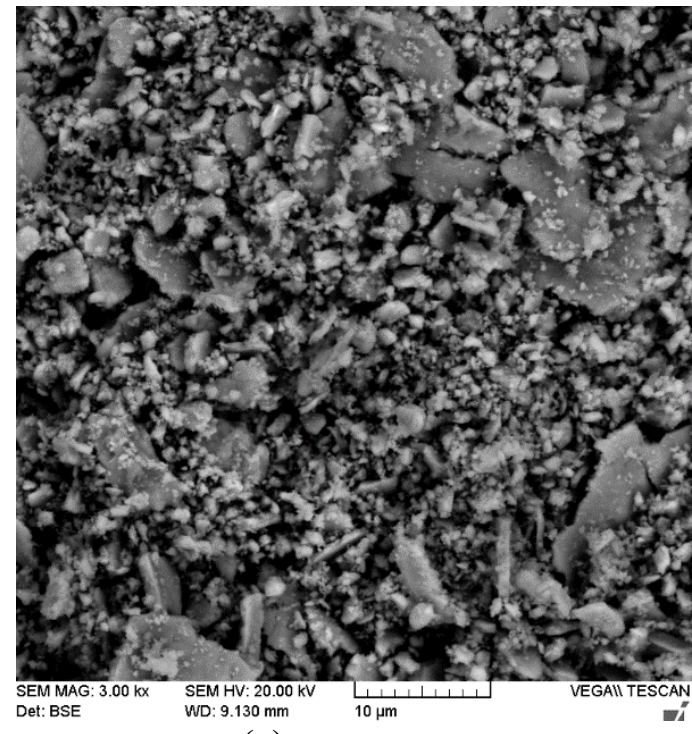

(a)

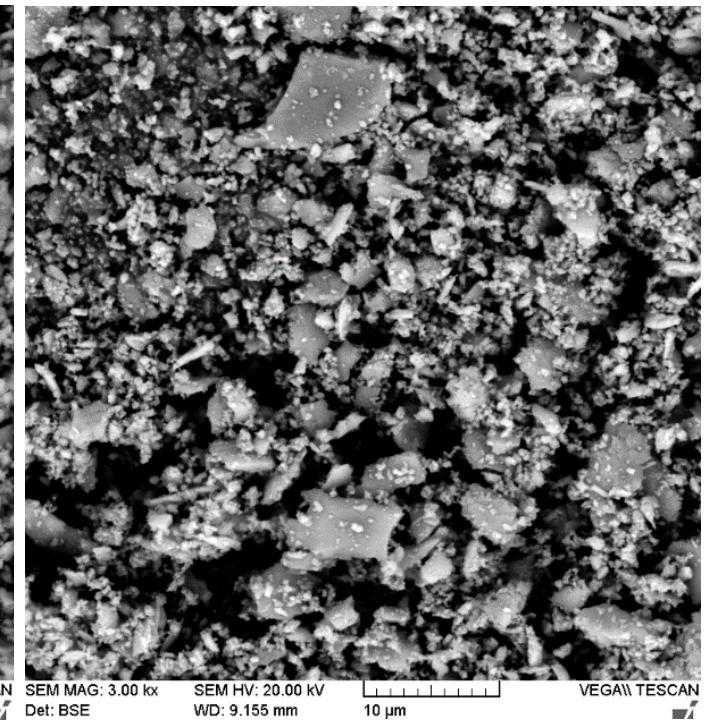

(b)

Figure 4-51: Stellite 728 wear debris at 25 N 60 rpm for (a) 1 hour, (b) 60 hours duration. 


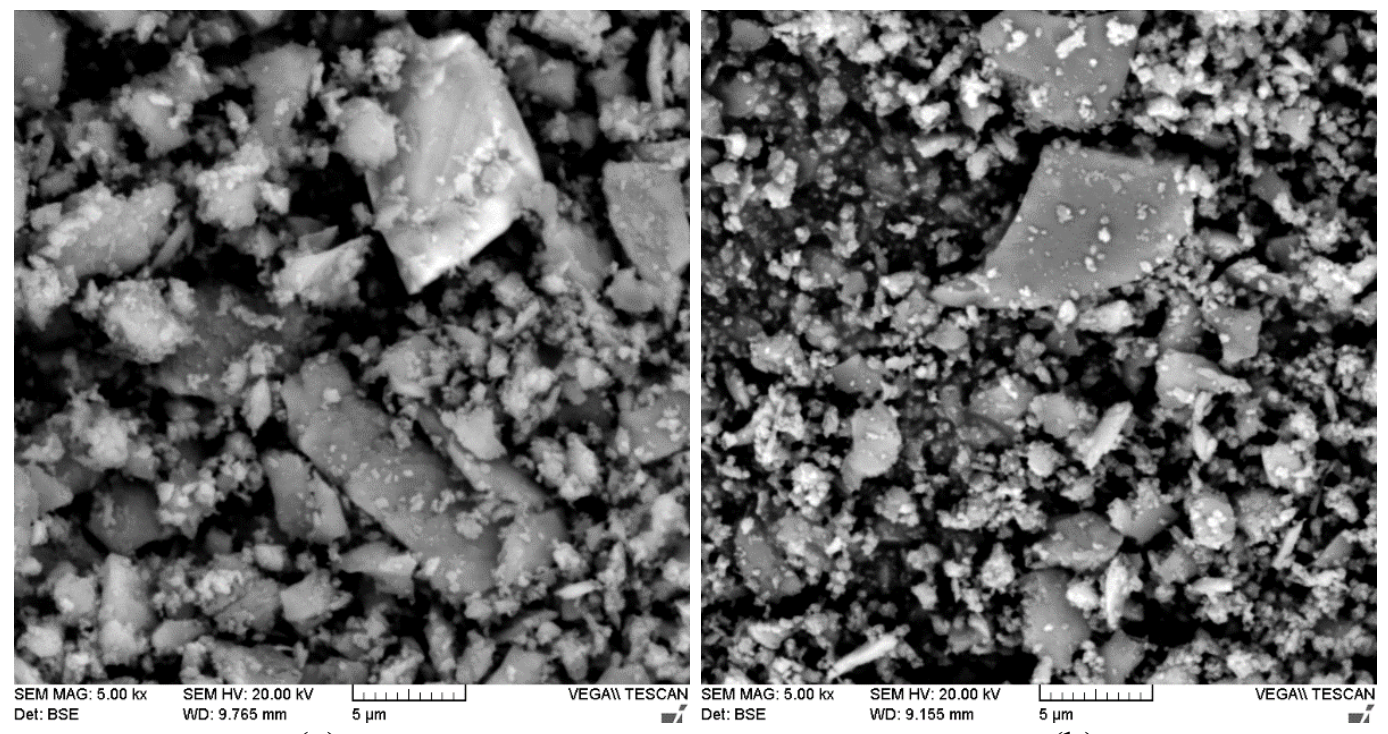

(a)

(b)

Figure 4-52: Wear debris for (a) Stellite 21 20hours, (b) Stellite 728 60hours duration.

Figure 4-53 shows a larger piece of debris that appears to have broken off the surface and remained relatively intact for SEM analysis. What also appeared noticeable is the staircase features of plastic wear on some of the particles.

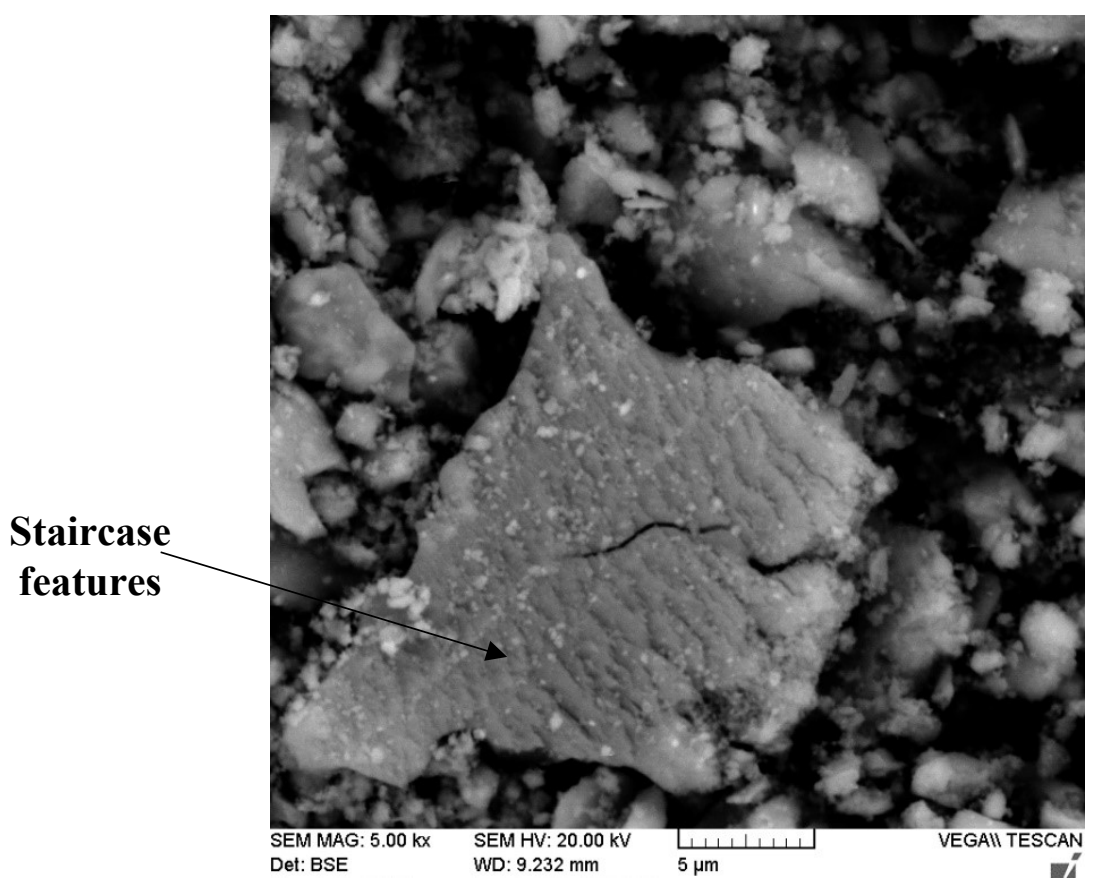

Figure 4-53: Stellite 720 wear debris from the test for $24 \mathrm{hrs}$. 


\subsubsection{EDX Data of Wear Debris}

Semi-quantitative SEM/EDX analysis was undertaken on the wear debris. Two analysis points were taken from each batch of debris collection, an example of the EDX spectrum site and reading are shown in Figure 4-54.

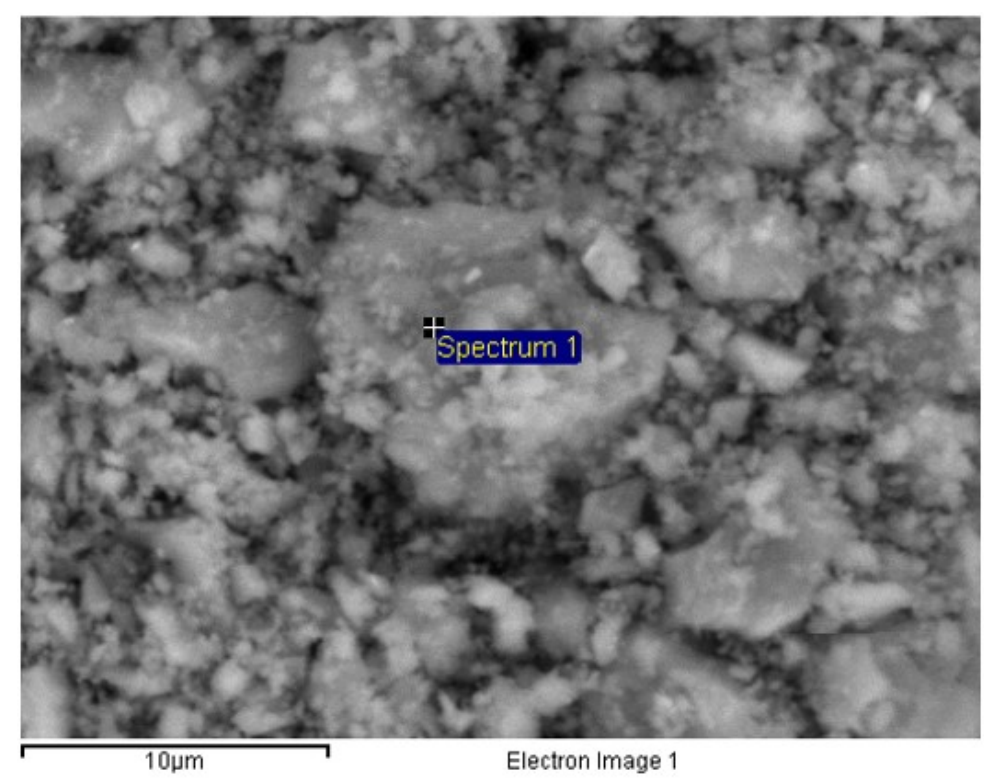

(a)

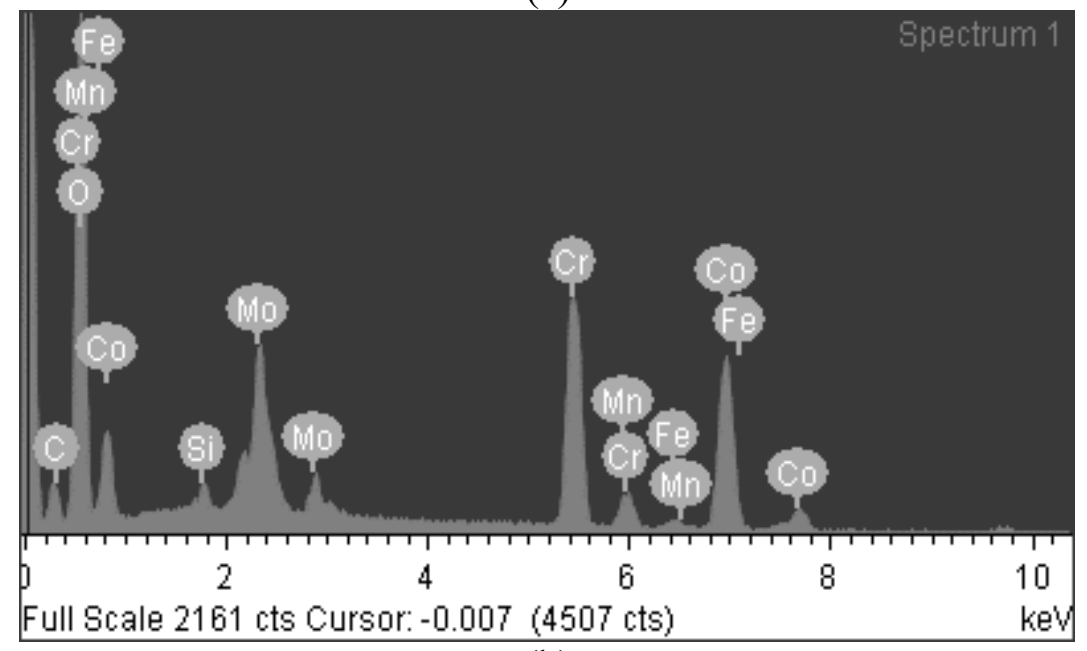

(b)

Figure 4-54: Debris EDX spectrum for Stellite 72060 rpm 25 N 60 hrs (a) analysis site, (b) spectrum. 
The variation of oxygen content with testing duration is illustrated in Figure 4-55. This data, although difficult to interpret because of the caveats to both EDX analysis techniques and the detection of oxygen, is primarily used to describe the oxidation process occurring in the wear track. A general trend across all three alloys shows that the oxygen content increases with duration during the initial readings up to $2 \mathrm{~km}$. However, after this point the oxygen appears to stabilize around the 35 to $45 \mathrm{wt} . \%$ range. This indicates that the oxide layer on the surface over time can oxidize further and eventually breaks away from the surface. It would be of interest to analyze debris from continuous testing to ascertain if a similar trend is seen.

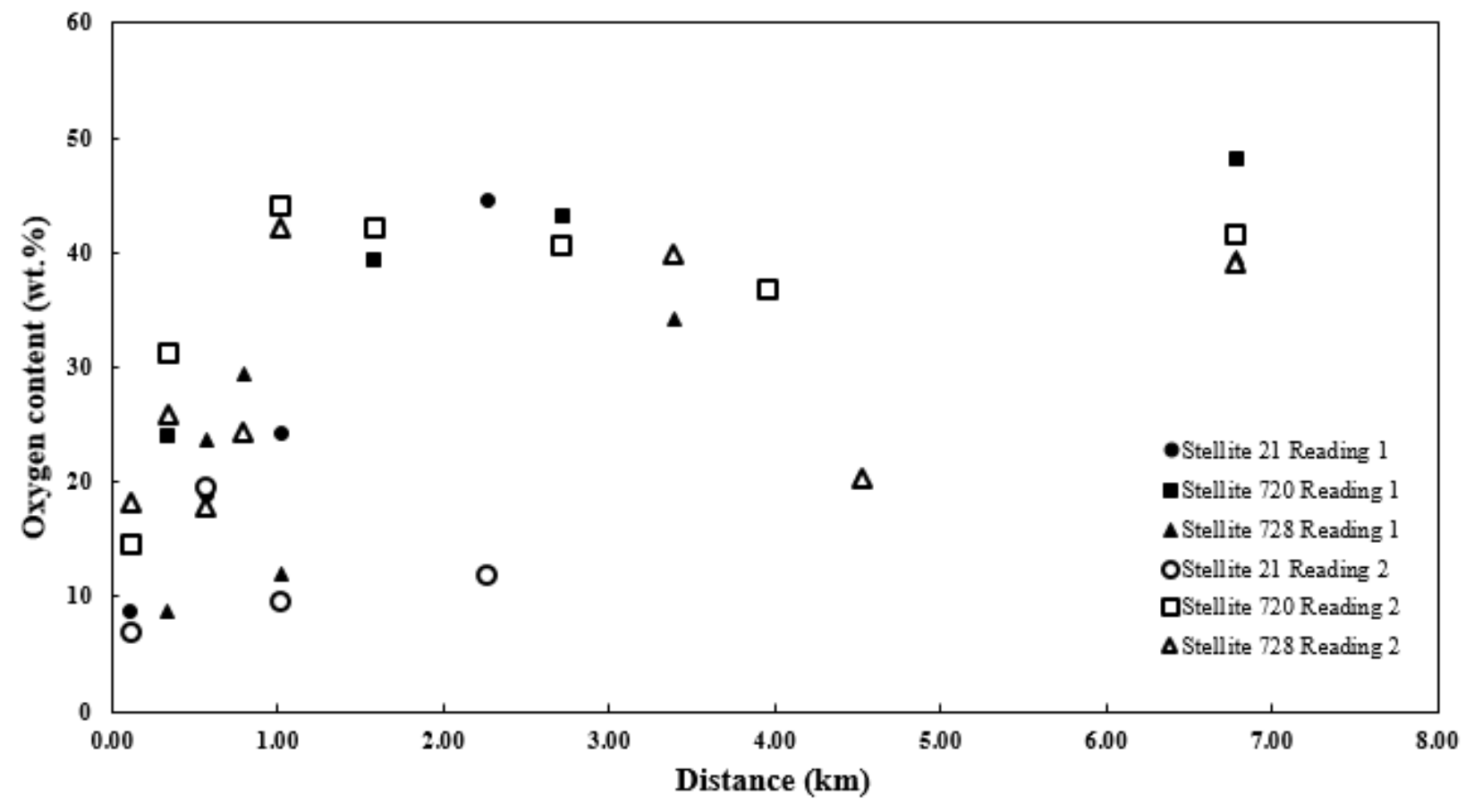

Figure 4-55: Oxygen content versus distance. 


\subsubsection{Wear Debris Particle Size Measurement}

The instrument ImageJ, version 51k was applied using the SEM images of the debris to analyze trends in particle size over the duration of testing. The method involved three steps: 1) Collect a sample of debris on a piece of conductive tape; 2) Take a SEM image of the sample; 3) Make a cross diagonally on the image; 4) Circle the particles lying on the cross; 3) Use the ImageJ to calculate the area of each particle on the cross automatically; and 4) Take the data and make an average. The debris sample from the test at $60 \mathrm{rpm}$-under $25 \mathrm{~N}$ was used to demonstrate the method of debris particle size measurement. Figure 4-56 shows an example of the line intercept and area measurement technique.

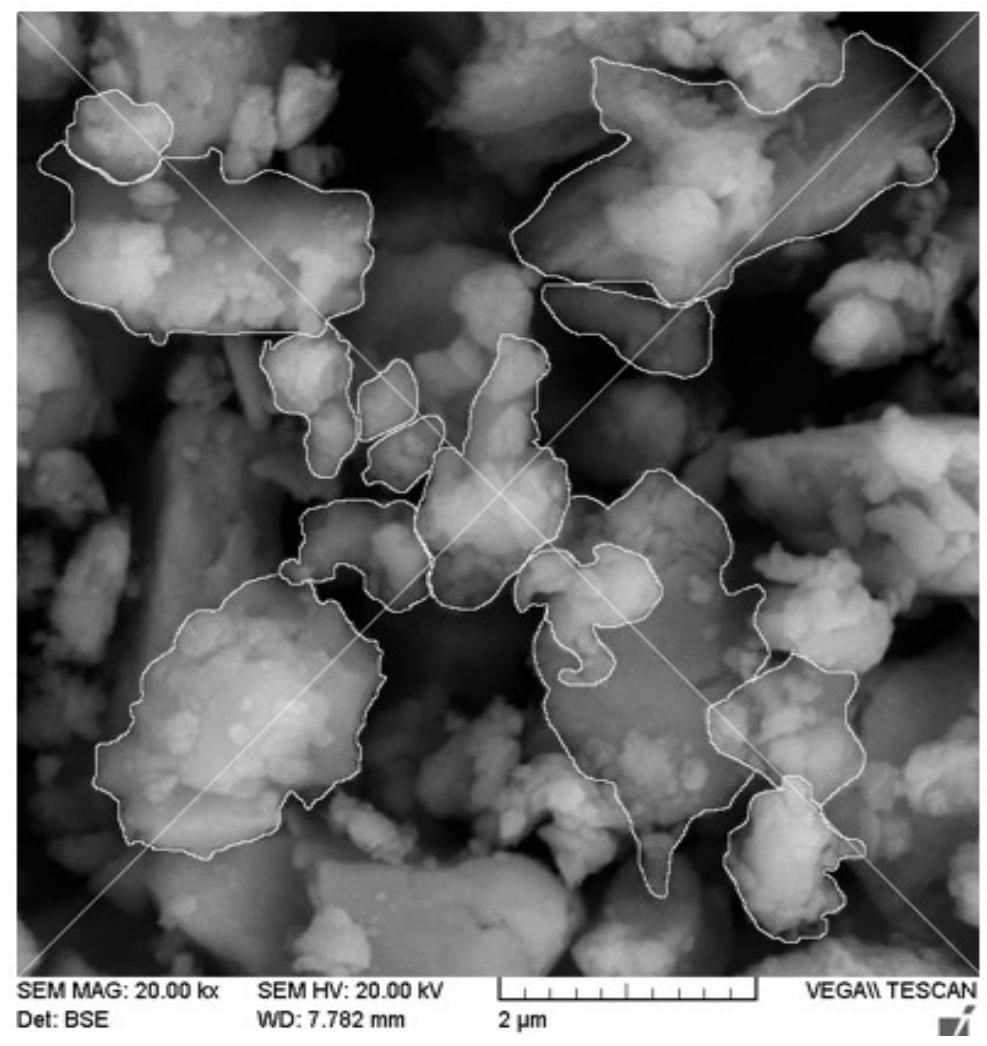

Figure 4-56: Particle size analysis of Stellite 21 wear debris from the test at $60 \mathrm{rpm}$ under $25 \mathrm{~N}$. 
It must be noted that a large portion of the particles appeared to show either heavy amalgamation of particles, and/or smaller particles attached to the surfaces of large particles, which made assessment using computer software difficult. These extremely fine particles may not be captured adequately in the interpretation and may skew the results in favour of large particles. However, even with the limited data from the Stellite 21 sample, and a potential to capture the larger particles with the software, a trend of decreasing particle size can be identified as the test duration increases, as summarized in Table 4-7.

Table 4-6: Average particle size $(\mu \mathrm{m})$ of wear debris from Stellite 21 specimen in interrupted test at $60 \mathrm{rpm}$ under $25 \mathrm{~N}$

\begin{tabular}{|c|c|c|c|c|}
\cline { 2 - 5 } \multicolumn{1}{c|}{} & \multicolumn{4}{c|}{ Debris interval check point } \\
\hline Alloy & $\mathbf{1 ~ h r}$ & $\mathbf{9}$ hrs & $\mathbf{4 0}$ hrs & $\mathbf{6 0}$ hrs \\
\hline Stellite 21 & 1.44 & 0.82 & N/A & N/A \\
\hline Stellite 720 & 1.91 & 1.61 & 0.95 & 0.67 \\
\hline Stellite 728 & 1.70 & 1.14 & 0.64 & 0.47 \\
\hline
\end{tabular}

As previously discussed, the volume loss is heavily influenced by the removal of wear debris in the system. Therefore, it could be expected that the size of the wear debris is also affected by removal of the particles. The particles appear to show amalgamation as shown in the staircasing profile and large particles breaking free (Figure 4-32c and Figure 4-53). This is an important note that should be considered when interpreting the results presented in Table 4-6.

\subsection{Modeling Study}

Modeling attempts to predict the wear loss of a material by a given set of circumstances within a wear system. This prediction can assist with reducing the number of physical wear tests, as well as predicting wear in similar materials and/or similar wear couples. In the present research physical data was obtained and used to model wear loss. 
In attempting to model the wear behaviour of the molybdenum-containing Stellite alloys, consideration was given to the trends shown by the volume loss curves over the distance of wear. For the molybdenum Stellite alloys Stellite 720 and Stellite 728, the majority of wear loss appears to occur in the first ten hours of testing, which is around one to two kilometers of distance travelled (Figure 4-14 to Figure 4-17). Thereafter, the volume loss appears to be mostly stabilized to the point where very little volume loss is experienced in both the continuous and interrupted testing. However, in the continuous testing at $50 \mathrm{rpm}$ under $25 \mathrm{~N}$, Stellite 21 shows a couple of transition points (Figure 4-14). Understanding these transitions and stabilized zones during the wear test is important for modeling.

\subsubsection{Modeling for Continuous Testing}

The volume loss evaluation shows that the transient stage is the most important to understand in the present research. The following equation can be proposed to model the wear being experienced by the Stellite alloys being studied:

$$
W=C_{1}\left(1-e^{-C_{2} L}\right)
$$

where $W$ is volumetric wear of the Stellite alloy, $C_{1}$ is a constant representing the total volume loss measured over the total distance, $C_{2}$ is a constant representing the transition point into the steady-state zone quantified as distance, and $L$ is the distance travelled. This preliminary model utilizes the Archard wear equation with wear test data fitting, and is based on the transient model (Eq. 2.10) proposed by Zhang et al. in their study of the running-in wear of a steel/SiC $\mathrm{p}_{\mathrm{p}} \mathrm{Al}$ 
composite system [27]. In the case of Stellite alloy materials and test methods, the constants have been grouped together attempting to understand the transient into steady-state relationship.

Stellite 720 and Stellite 728 show minimal volume loss after the transient stage. Stellite 21, on the other hand, shows an increased volume loss as the distance increases under $25 \mathrm{~N}$ loading (Figure 4-14). A further equation is proposed to model Stellite 21 with an additional term to address the linear relationship exhibited by this alloy as the distance increases:

$$
W=C_{1}\left(1-e^{-C_{2} L}\right)+K_{1} L
$$

where $K_{1}$ is the slope of the linear wear loss in the steady-state stage.

The best fit model for the continuous testing at $50 \mathrm{rpm}$ under $15 \mathrm{~N}$ and $25 \mathrm{~N}$ is presented in Figure 4-56 and the parameter values are given in Table 4-8.

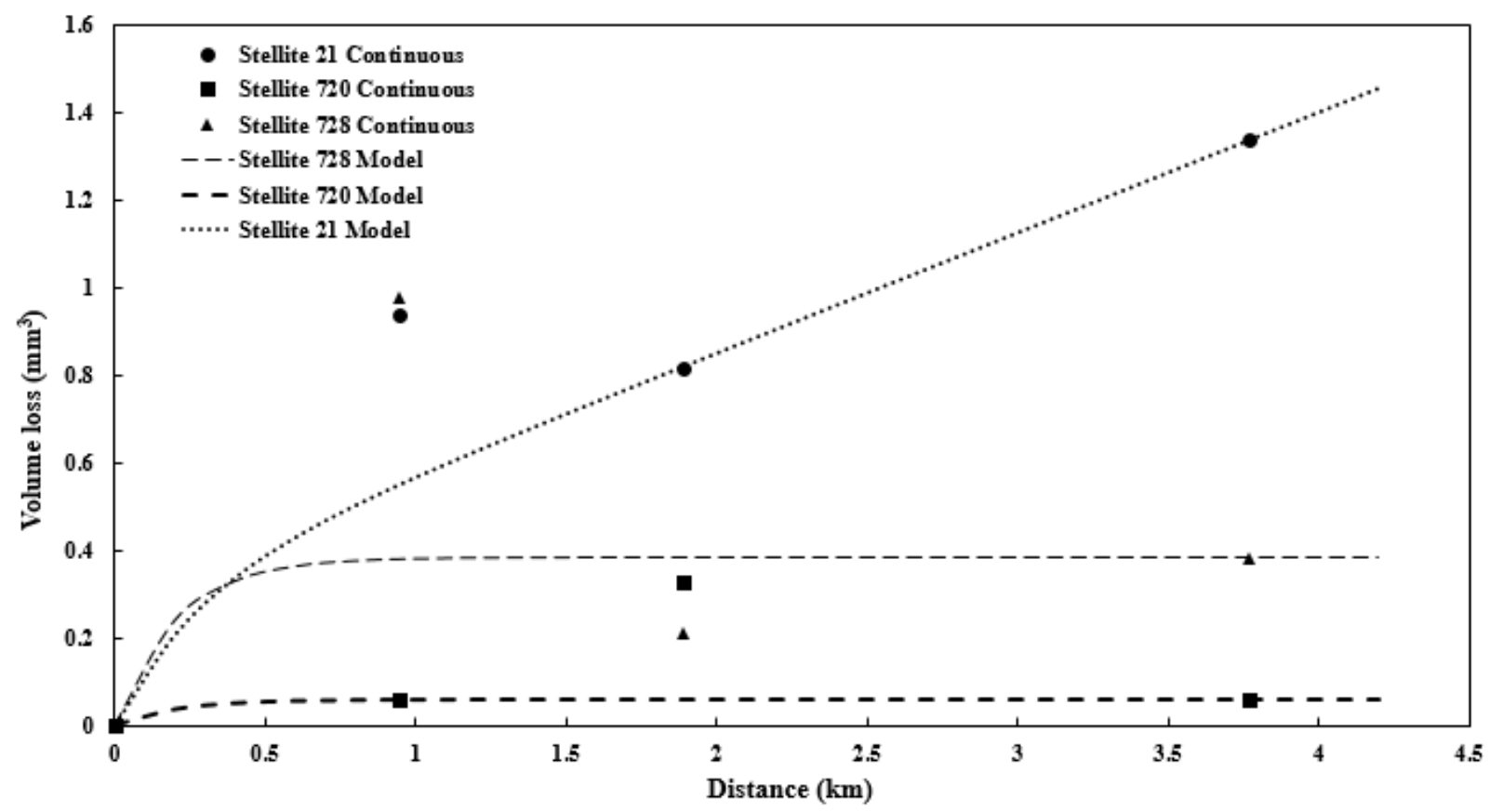

(a) 


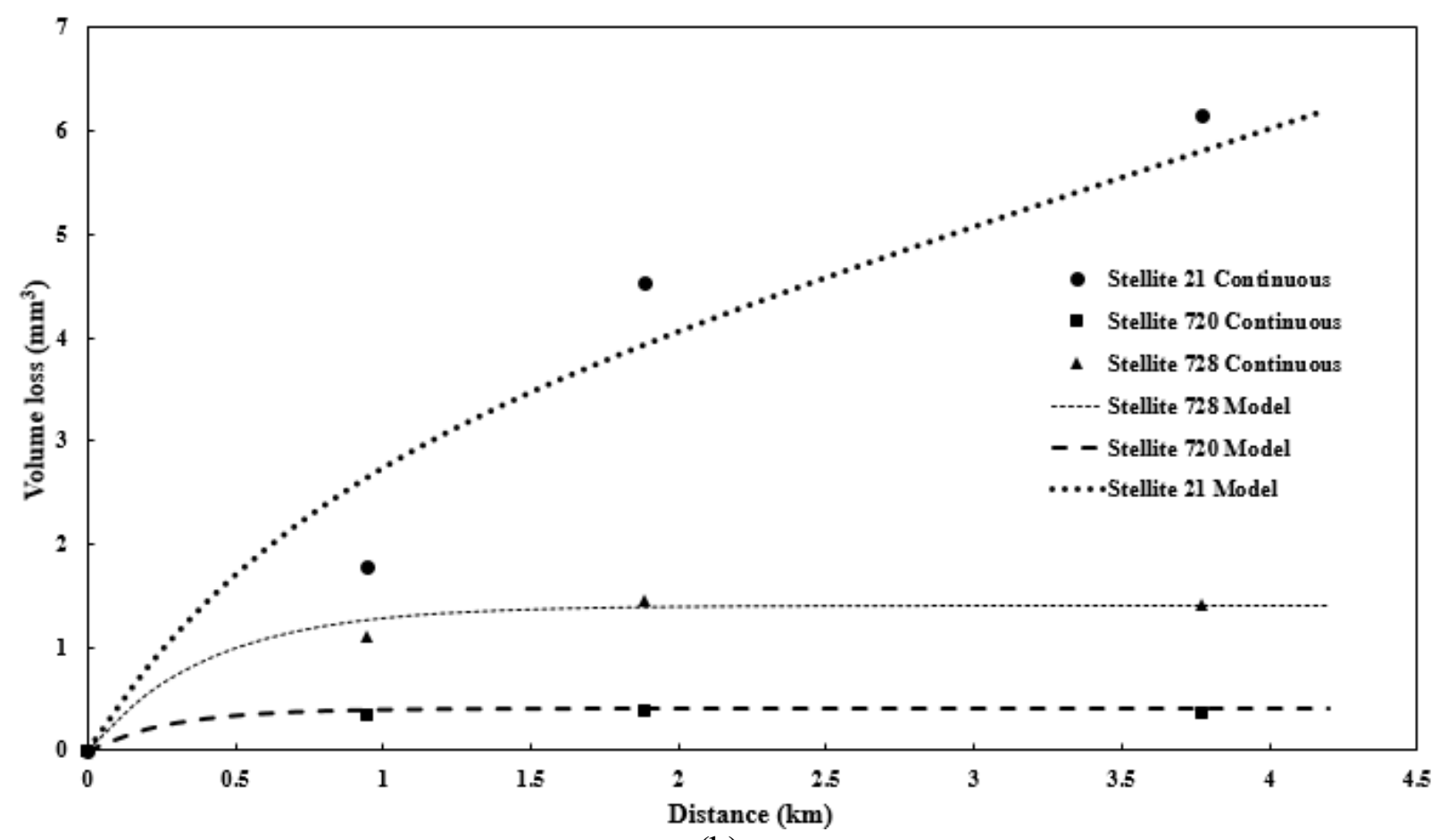

(b)

Figure 4-57: Modeling results for continuous testing at $50 \mathrm{rpm}$ (a) under $15 \mathrm{~N}$, (b) under $25 \mathrm{~N}$.

Table 4-7: Mode constants best-fit data for continuous testing

\begin{tabular}{|c|c|c|c|c|}
\hline \multirow{2}{*}{$\begin{array}{c}\text { Normal Load } \\
(\mathbf{N})\end{array}$} & \multirow{2}{*}{ Alloy } & \multicolumn{3}{|c|}{ Constant } \\
\cline { 2 - 5 } & & $\boldsymbol{C}_{\mathbf{1}}$ & $\boldsymbol{C}_{\mathbf{2}}$ & $\boldsymbol{K}_{\mathbf{1}}$ \\
\hline \multirow{3}{*}{$15 \mathrm{~N}$} & Stellite 21 & 0.30 & 1.30 & 0.275 \\
\cline { 2 - 5 } & Stellite 720 & 0.06 & 0.94 & $\mathrm{~N} / \mathrm{A}$ \\
\cline { 2 - 5 } & Stellite 728 & 0.38 & 0.94 & N/A \\
\hline \multirow{3}{*}{$25 \mathrm{~N}$} & Stellite 21 & 2.30 & 3.00 & 0.93 \\
\cline { 2 - 5 } & Stellite 720 & 0.40 & 1.30 & N/A \\
\cline { 2 - 5 } & Stellite 728 & 1.40 & 1.88 & N/A \\
\hline
\end{tabular}

The continuous testing data for $15 \mathrm{~N}$ and $25 \mathrm{~N}$ show two relationships, 1) primarily an exponential relationship for Stellite 720 and Stellite 728, and 2) exponential plus linear for Stellite 21. The behavior of Stellite 21 in particular shows a greater complexity having two components in the volume loss data set. The first is an exponential relationship in the transient stage, and the second is a linear relationship in the steady-state zone. Stellite 720 and Stellite 728 show only an 
exponential relationship in the transient stage, and the volume loss appears to approach a constant level.

Continuous testing data for $15 \mathrm{~N}$ loading show that both the model and data are somewhat limited in interpretation. The limited data points and scatter between the durations do not allow adequate conclusions to be made. The first point to note is that the data for Stellite 21 and Stellite 728 under $15 \mathrm{~N}$ shows a disagreement with the volume loss experienced at longer distances. The two points at the $\sim 1 \mathrm{~km}$ mark can be considered outliers with respect to the best-fit modeling. This is indicative of the ploughing identified in the early stages, and then some recovery in the form of a staircasing type mode of solid solution build-up on the surface. The best fitting value of $C_{1}$, a constant to indicate that the total volume loss appears to be highest for both Stellite 728 at 0.38 , and Stellite 21 at 0.30 . This result is reflected in the SEM worn surface analysis where Stellite 21 and Stellite 728 show scoring and ploughing on the surface at the $10 \mathrm{hrs}$ stage (Figure 4-24 and Figure 4-40 respectively.). However, for the longer durations, SEM worn surface analysis of Stellite 728 shows that the carbides are more pronounced with a build-up of wear debris adjacent to the carbides (Figure 4-43), suggesting the wear debris lost early in the process adheres to the surface to protect/prevent further wear loss. The continued volume loss of Stellite 21 is in keeping with the low level of carbides and more solid-solution breaking down as the duration increases. This is represented by $K_{1}$ as the slope of the linear wear in the steady-state stage. No adjustment is made to best fit $K_{1}$ for Stellite 21. For the steady-state transition constant $C_{2}$, this again is difficult to interpret for all of the alloys at $15 \mathrm{~N}$ due to the limited data set and the fluctuations in the volume loss. An important consideration for the $15 \mathrm{~N}$ testing data is that the volume loss is not very significant, $<1 \mathrm{~mm}^{3}$ for most of the specimens across all three alloys. In this respect, modeling the behaviour at such a low level of volume loss will be difficult because of scatter in the results. 
The data for the $25 \mathrm{~N}$ test appears to follow more of the expected outcomes to differentiate between the alloys based on volume loss and volume fraction of carbides. Stellite 720 having the highest volume fraction of carbides shows the lowest value of $C_{1}$, and Stellite 21 having the lowest volume fraction of carbides shows the highest value of $C_{1}$. Stellite 728 falling between the other two alloys shows a middle ground value which is in keeping with the carbide volume fraction. However, the best fit model for Stellite 21 requires a reduction in the value of $C_{1}$ by more than half, which demonstrates that the model predicts too high of an expected volume loss based on actual data. The value of $C_{2}$ also requires adjustment to address the steady-state transition point. Stellite 720 and Stellite 728 appear to show an easily identifiable steady-state transition point with very little volume loss occurring after this point. The as-modeled $C_{2}$ value is increased slightly for Stellite 720 and Stellite 728 to model the steady-state developing at a longer than predicted duration. For Stellite 21, the value of $C_{2}$ is more than doubled to increase the transition into steadystate, which is difficult to discern from the data set. However, the best fit for $K_{1}$ is not adjusted, and compared to the $15 \mathrm{~N}$ data set, the $25 \mathrm{~N}$ data set shows an increase in $K_{1}$ for Stellite 21, which follows for the higher load to cause increased wear loss over the same duration.

The main concern with evaluating and modeling the data is both the limited data set and loading level on the system. In order to test the model a higher load and/or rpm would be required to break down and really test the alloy to show significant volume losses and prove out the model.

\subsubsection{Modeling for Interrupted Testing}

In terms of the transient and steady-state wear transitions, during interrupted testing, all three alloys showed similar trends for volume loss when tested at $50 \mathrm{rpm}$ and $60 \mathrm{rpm}$ with a $25 \mathrm{~N}$ 
load (Figure 4-17). The wear model previously discussed has been applied to the interrupted testing data (Figure 4-58 and Figure 4-59), and the best fitting data are presented in Table 4-9.

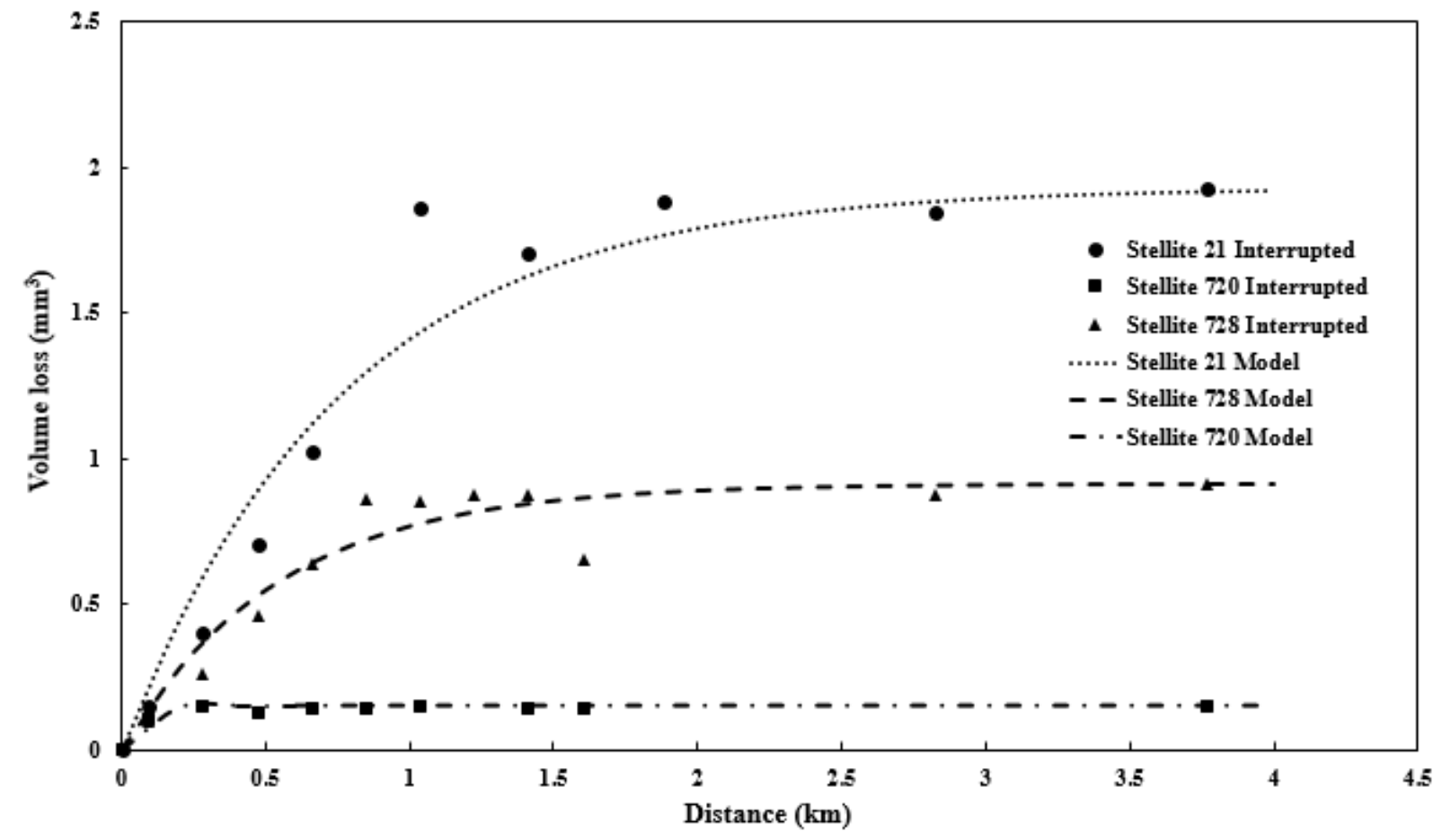

Figure 4-58: Modeling of interrupted test at $50 \mathrm{rpm}$. 


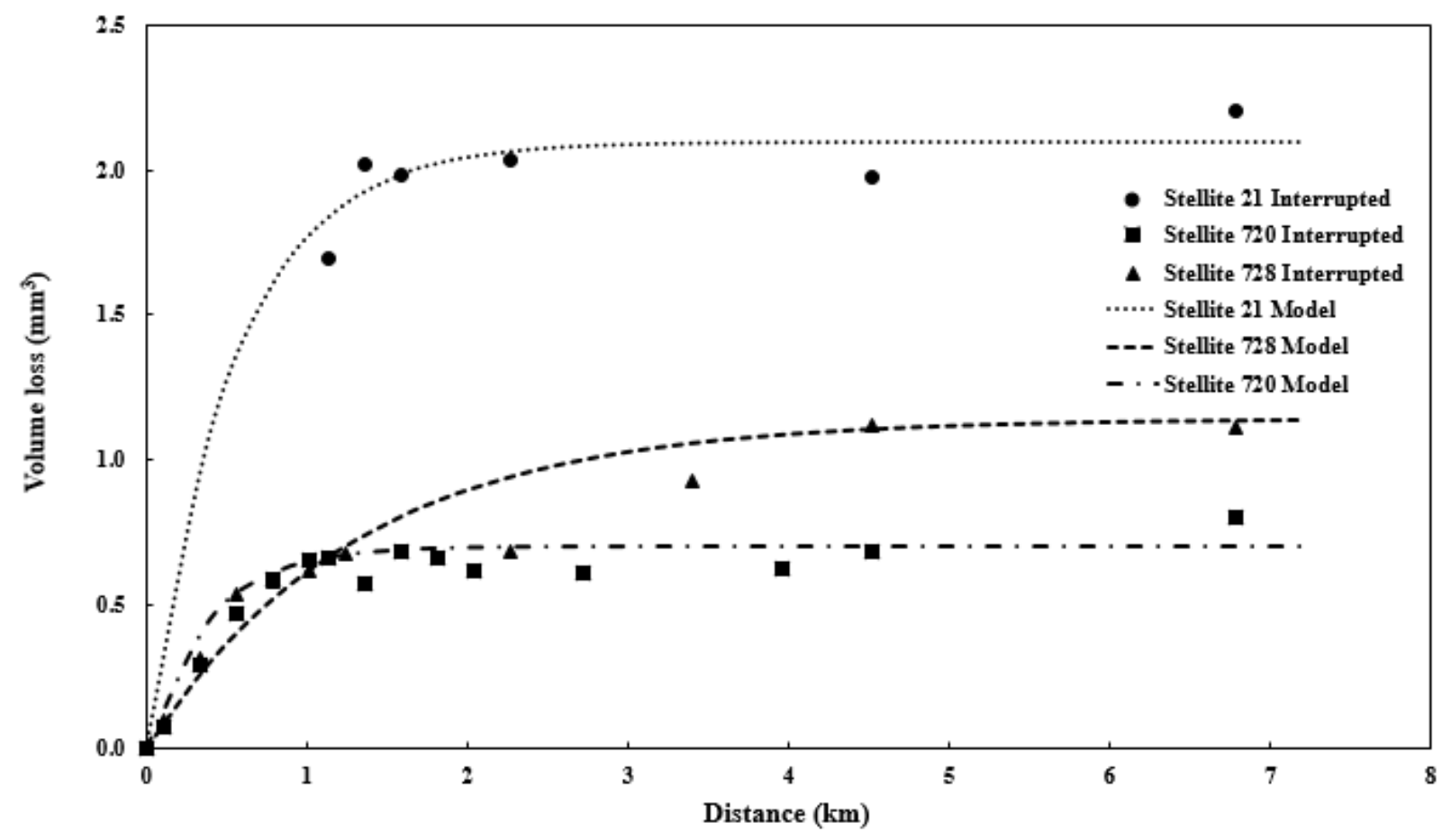

Figure 4-59: Modeling of interrupted test at $60 \mathrm{rpm}$.

Table 4-8: Model constants best-fit data for interrupted testing

\begin{tabular}{|c|c|c|c|}
\hline \multirow{2}{*}{ Speed (rpm) } & \multirow{2}{*}{ Alloy } & \multicolumn{2}{|c|}{ Constant } \\
\cline { 3 - 4 } & & $\mathbf{C}_{\mathbf{1}}$ & $\mathbf{C}_{\mathbf{2}}$ \\
\hline \multirow{3}{*}{50} & Stellite 21 & 1.93 & 3.5 \\
\cline { 2 - 4 } & Stellite 720 & 0.15 & 0.28 \\
\cline { 2 - 4 } & Stellite 728 & 0.91 & 2.5 \\
\hline \multirow{3}{*}{60} & Stellite 21 & 2.1 & 2.5 \\
\cline { 2 - 4 } & Stellite 720 & 0.7 & 1.8 \\
\cline { 2 - 4 } & Stellite 728 & 1.14 & 6 \\
\hline
\end{tabular}

The interrupted testing of Stellite 21 did not show a linear volume loss in the steady-state zone, instead it followed similar trends as that exhibited by Stellite 720 and Stellite 728 . The majority of as-modeled $C_{1}$ values did not need any adjustment. However, a best-fit was required for the as-modeled $C_{2}$ constant which predicted an earlier transition to steady-state than that found by actual interrupted testing. Stellite 728 in particular does not show a sharp transition into steady- 
state as that exhibited by Stellite 21 and Stellite 720 . This transition delay could be reflected in the type of carbides present in Stellite 728. Niobium being added to the composition creates potentially more stable niobium type carbides which may show more of a resistance to pulling out and/or breaking down in the microstructure during the sliding wear process. This is represented by minimal cracking of the carbide structure in Stellite 728 as shown in Figure 4-48.

A summary of the modeling results is illustrated in Figure 4-60 where $C_{1}$ is plotted against hardness. This shows for the continuous testing at $15 \mathrm{~N}$ (Figure 4-60a) the hardness does not significantly affect the constant, and the $C_{1}$ values are similar across all three alloys even though they are all very different in terms of their hardness and microstructure. In contrast to this, the $C_{1}$ values for continuous testing at $25 \mathrm{~N}$ show a more linear relationship in terms of a decreasing $C_{1}$ value as hardness increases. This data could prove useful for predicting wear for other molybdenum Stellite alloys having different hardness values in between the three chosen alloys.

The interrupted testing at $50 \mathrm{rpm}$ and $60 \mathrm{rpm}$ with a $25 \mathrm{~N}$ load showed similar trends to the continuous at $25 \mathrm{~N}$. Stellite 21 and Stellite 728 appear to show a similar decrease in $C_{1}$ value for both speeds (Figure 4-60b). However, Stellite 720 showed a slight difference suggesting the increased speed affects the wear mechanism (Figure 4-60b). 


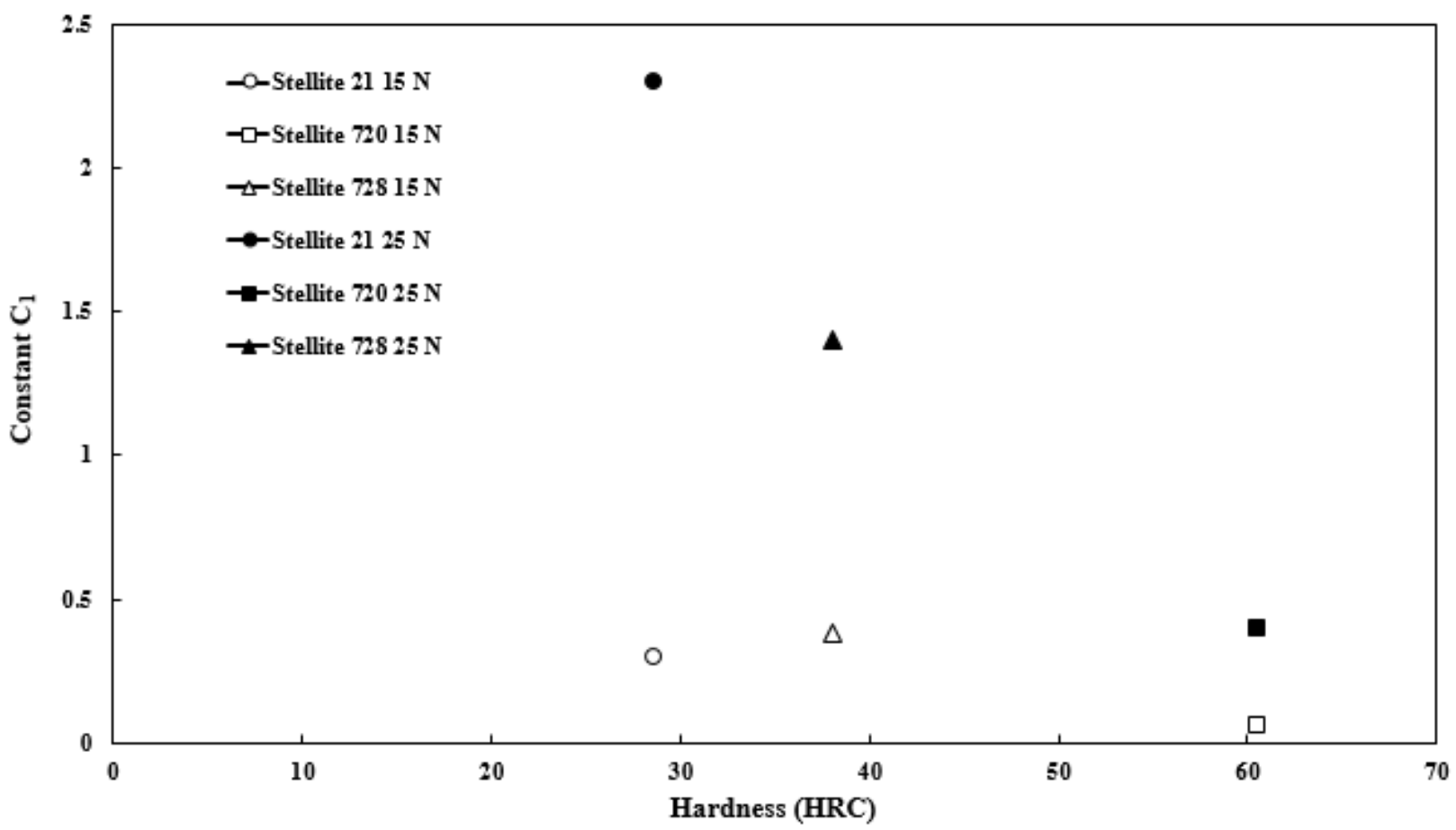

(a)

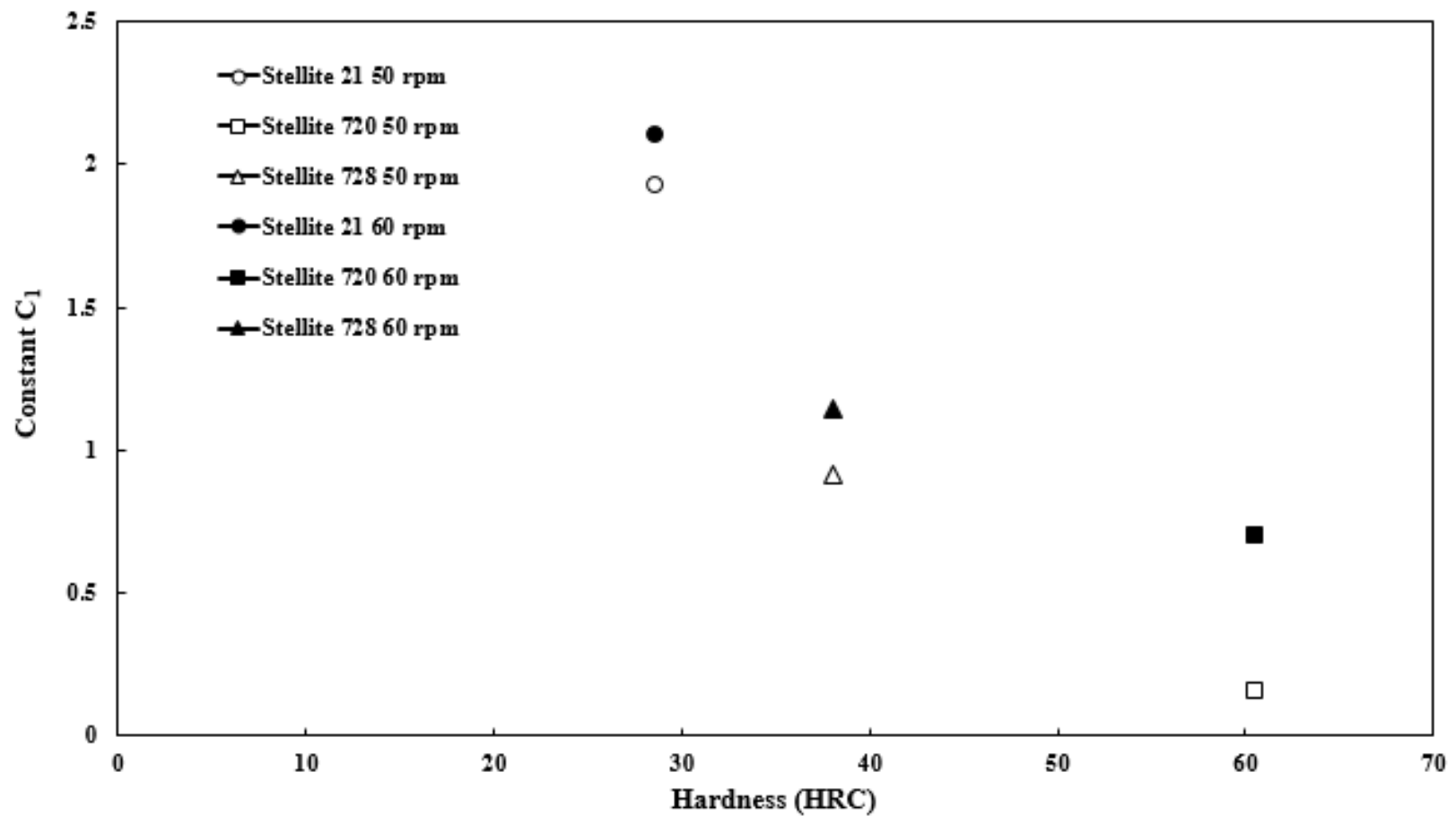

(b)

Figure 4-60: $C_{1}$ value vs. hardness (a) Continuous testing at $50 \mathrm{rpm}$ under $15 \mathrm{~N}$ and $25 \mathrm{~N}$, (b) Interrupted testing at $50 \mathrm{rpm}$ and $60 \mathrm{rpm}$ under $25 \mathrm{~N}$. 


\subsection{Discussion}

In performing the wear testing an obvious difference between continuous and interrupted testing can be established. It is important to distinguish that the key difference is the removal of debris from the system in the interrupted testing. This validates how the wear mechanism can change from one mode to another. Removing debris showed lower average friction coefficients, lower wear coefficients, and lower volume losses than compared to the continuous testing under the same conditions.

In assessing the volume loss, the test at $50 \mathrm{rpm}$ with a load of $15 \mathrm{~N}$ under continuous testing conditions revealed minimal volume loss. No significant trends could be identified with this data set due to such low volume losses. When the load was increased to $25 \mathrm{~N}$, trends could be identified in the continuous data set. The largest volume loss appeared to occur during the first $1.5 \mathrm{~km}$ for all three alloys (Figure 4-14). This transient zone could be considered an initial running-in period which eventually led to a more protective oxide layer forming on the surface to combat wear thus creating a more steady-state zone. Based on the volume fraction of carbides, Stellite 21 having $\sim 4 \%$, Stellite 720 having $\sim 70 \%$, and Stellite having $728 \sim 21 \%$, it would be expected to see Stellite 21 to have the highest volume loss, and Stellite 720 to have the lowest, with Stellite 728 falling somewhere in between the two. This result was found in the $25 \mathrm{~N}, 50 \mathrm{rpm}$ continuous test data set (Figure 4-14).

The volume loss for the interrupted testing was interesting in terms of the similarities between Stellite 21 and Stellite 728 . At $50 \mathrm{rpm}$ and $60 \mathrm{rpm}$, Stellite 21 showed a similar volume loss profile for both the $50 \mathrm{rpm}$ and $60 \mathrm{rpm}$ test conditions (Figure 4-17), and the same can be observed for Stellite 728. This indicates that the change from $50 \mathrm{rpm}$ to $60 \mathrm{rpm}$ does not change the wear mechanism significantly. However, for Stellite 720 the wear loss increases as the speed 
is increased from $50 \mathrm{rpm}$ to $60 \mathrm{rpm}$. This indicates that some change is starting to occur with an increased rpm. This could possibly be related to breaking down of the large carbides (Figure 439). However, it should be kept in mind that the overall wear loss for this alloy is still very minimal $<1 \mathrm{~mm}^{3}$.

Review of the friction coefficient for each of the alloys under the continuous testing conditions shows that the average value increases as the duration increased (Figure 4-18). Overall Stellite 21 at $25 \mathrm{~N}$ shows the lowest friction coefficient at the longest duration. The three Stellite alloys at $15 \mathrm{~N}$ also shows very similar values at each of the durations tested at $15 \mathrm{~N}$. This data reveals that the $15 \mathrm{~N}$ normal load does not cause too much in the way of friction changes during the testing. The higher friction coefficient values are seen at the higher load of $25 \mathrm{~N}$ for Stellite 720 and Stellite 728 . This would be expected in terms of the potential of abrasive particles to be introduced in the wear path having been separated from the surface.

In assessing the friction coefficient for the interrupted testing conditions, the removal of wear particles caused each alloy to have a reduced and steady average friction coefficient compared to the continuous testing (Figure 4-19). An interesting feature of the friction coefficient data from the $50 \mathrm{rpm}$ and $60 \mathrm{rpm}$ interrupted testing data is that Stellite 728 has the lowest value in both instances (Figure 4-20). This suggests that the remaining wear particles from the Stellite 728 and/or the oxide layer somehow contribute to a lower coefficient of friction than those from Stellite 21 and Stellite 720. In the case of Stellite 728, the major difference is the niobium addition which involves the formation of niobium carbides and intermetallics. The hardness and properties of the oxide layer appear to lend themselves to reducing the average friction coefficient which is a good attribute in wear processes, particularly in unlubricated conditions. 
The Archard equation was used to calculate the wear coefficient for each of the Stellite alloys in the present study. The $25 \mathrm{~N}$ test specimen show a greater wear coefficient than the $15 \mathrm{~N}$ test ones for the continuous testing conditions (Figure 4-21) which is to be expected given the higher load. In relation to the volume fraction of carbides, the wear coefficient shows the expected results for the three alloys. Stellite 21 having $\sim 4 \%$ volume fraction of carbides has the highest wear rate, Stellite 720 having $\sim 70 \%$ has the lowest wear rate, and Stellite 728 having $\sim 21 \%$ falls between the previous two alloys. This also holds true for the $25 \mathrm{~N}, 50 \mathrm{rpm}$ and $60 \mathrm{rpm}$ interrupted testing conditions. However, an exception to this is Stellite 720 when the test was run at $60 \mathrm{rpm}$ under a $25 \mathrm{~N}$ load. As seen in the friction coefficient data, Stellite 720 underwent a change during interrupted testing from $50 \mathrm{rpm}$ to $60 \mathrm{rpm}$.

An important feature shown in the wear surface analysis is the formation of a worm-like shape to the wear track. This feature stems from a build-up of wear debris occurring at intermittent areas along the wear track. Analysis of the worn surfaces clearly shows ploughing and scoring of the surface in the samples for Stellite 21. This is related to the low volume fraction of carbides to resist wear. The resulting debris forms mostly from the solid solution and becomes embedded on the surface in the form of staircasing as plastic deformation continues to occur. The oxide layer on the Stellite 21 samples does not show the extensive network of cracking as that shown on Stellite 720 and Stellite 728. Another interesting feature in Stellite 720 (Figure 4-34b) and Stellite 728 (4$45 \mathrm{~b}$ ) is the build-up of wear debris at the carbides. In Stellite 720, this would be expected because of the large size of the carbide. In Stellite 728 , the hardness of the carbide and the size will influence the behaviour of the alloy.

The analysis of the wear debris from the interrupted $25 \mathrm{~N}, 60 \mathrm{rpm}$ testing at $25 \mathrm{~N}, 60 \mathrm{rpm}$ showed a general increase in oxygen content for each of the alloys. The caveats associated with 
this type of analysis prevent solid conclusions to be made. However, for this set of results, as the duration increases, it is expected that more oxidation will occur in the wear debris.

In terms of modeling of the volume loss, the continuous testing at $50 \mathrm{rpm}$ and $15 \mathrm{~N}$, did not show a significant difference in volume loss between each of the alloys as the duration increases. Therefore, modeling was difficult to accomplish (Figure 4-57a). However, for the samples of the continuous testing at $50 \mathrm{rpm}$ under $25 \mathrm{~N}$, a difference between the alloys can be clearly identified in the volume loss as the duration increases (Figure 4-57b). Stellite 720 and Stellite 728 show a steady-state from $\sim 1 \mathrm{~km}$ duration, and Stellite 21 shows a linear steady-state volume loss as the duration increases. This difference results in a different model being required to fit Stellite 21 from that identified for Stellite 720 and Stellite 728. In contrast to the continuous testing conditions, the interrupted testing shows a similar trend between all three alloys for both $50 \mathrm{rpm}$ and $60 \mathrm{rpm}$. Therefore, one equation could be used with a change only to the constant values with different values for the two constants. The modeling for the interrupted testing showed that the important transition from transient to steady-state is the key to establishing a good model. 


\section{Conclusions and Future Work}

\subsection{Conclusions}

In the present study, an unlubricated sliding wear test was conducted on three molybdenum-containing Stellite alloys using a pin-on-disc tribometer with a WC ball. The properties of Stellite alloys are primarily attributed to the carbon content and hence the volume fraction of carbides forming in the alloy. Other alloying elements such as chromium and molybdenum also contribute to the formation of the carbides and solid solution properties. Three molybdenum Stellite alloys: Stellite 21, Stellite 720 and Stellite 728, were chosen based on the carbon content ranging from $0.25 \sim 2.5$ wt.\%, chromium content between $24 \sim 33$ wt. $\%$, molybdenum content between $5.5 \sim 18$ wt.\%, and one alloy, Stellite 728 , has the addition of 2 wt.\% niobium. It was shown that these alloys have varying degrees of volume fraction of carbides. Stellite 21 having $\sim 4 \%$, Stellite 720 having $\sim 70 \%$, and Stellite having $728 \sim 21 \%$. It would be expected to see Stellite 21 to have the highest volume loss, and Stellite 720 to have the lowest, with Stellite 728 falling somewhere in between the two.

Data in the form of friction coefficients, wear coefficients, wear debris analysis and worn surface analysis were obtained to provide different perspectives in order to evaluate the samples. Through analyzing the wear data, a basic wear model was applied to identify common features that may be applicable to predicting wear in molybdenum Stellite alloys. An extensive study of the microstructure was also undertaken to establish the type of carbides and intermetallics present in the three alloys being studied. The following conclusions can be made from the present research: 
1) The findings in this study built on the microstructural analysis of the Stellite molybdenum alloys. A greater understanding of the various and complex carbides and intermetallics has been presented.

2) A load of $15 \mathrm{~N}$ at $50 \mathrm{rpm}$ did not show significant volume loss for any of the Stellite alloys tested.

3) The greatest degree of wear, i.e. volume loss, appeared to occur within the first $1.5 \mathrm{~km}$ of the wear testing for all three alloys under the majority of wear conditions. This can be considered the transient state.

4) The steady-state appeared to show very little volume loss for Stellite 728 and Stellite 720 under both continuous and interrupted testing conditions. Stellite 21 showed a greater rate of wear under the continuous conditions at $25 \mathrm{~N}$ load and $50 \mathrm{rpm}$.

5) In terms of total volume loss, the interrupted testing showed a lesser degree of volume loss on the system due to the removal of the wear debris compared to the continuous testing.

6) Under $25 \mathrm{~N}$ at $50 \mathrm{rpm}$ the volume loss for the continuous and interrupted testing appeared to show similar volume loss rates up to $1.5 \mathrm{~km}$. This suggests that even after the debris was removed for the interrupted testing, the surfaces of the alloys underwent some arbitrary running-in process to establish a steady-state.

7) Analysis of the wear surfaces revealed a primarily staricasing feature from the constant plastic deformation of broken asperities falling into the wear path. This layer of plastically deformed debris also showed a network of cracks which resulted in pieces breaking away and falling into the wear path. 
8) The wear debris appeared to adhere to the large blocky Cr-rich carbide in Stellite 720, and on the lamellar eutectic carbides in Stellite 728. Minimal cracking of the carbides was observed in both of these alloys.

9) Analysis of the wear debris is a relatively new topic for discussion in the wear of molybdenum Stellite alloys. SEM results of the debris showed the staircasing features to be intact within the debris, and an attempt to investigate the oxygen content was undertaken using SEM/EDX to find trend between the data sets. A general conclusion would be that oxygen increased over the duration of testing for each of the alloys.

10) Particle size measurement was also attempted on the wear debris for the interrupted testing condition. As the duration increased, a general trend of smaller particles being generated was found.

11) A wear model was developed for both the continuous, and interrupted testing condition. The models show good correlation with the data sets. The continuous wear loss during testing at 50rpm under $15 \mathrm{~N}$ was generally low but with large scatter (outliers).

12) The constant signifying the volume loss in the wear model was found to be dependent on the hardness of the alloys, with a particular trend to decreasing value with increasing hardness in the continuous testing at $50 \mathrm{rpm}$ under $25 \mathrm{~N}$, and the interrupted testing under $25 \mathrm{~N}$ at $50 \mathrm{rpm}$ and $60 \mathrm{rpm}$. The $15 \mathrm{~N}$ test data set did not show clear correlations due to the minimal volume loss on each of the alloys. 


\subsection{Future Work}

The degree of testing and evaluation undertaken in the present work has paved a way for a variety of different approaches to studying the behaviour of molybdenum Stellite alloys under unlubricated sliding wear conditions:

1) Validating the continuous test conditions and obtaining more data points to establish the relationship of the model performance with the wear condition parameters.

2) Performing more continuous tests with Stellite 21, Stellite 720 and Stellite 728 at closer intervals to differentiate the dominant wear mechanisms at different wear stages.

3) Testing other molybdenum Stellite alloys, such as Stellite 706, Stellite 712 (Table 2-1) under the same conditions to validate the model using Figure 4-60 to predict the constant.

4) Running increased levels of normal load and rpm using the same Stellite alloys and establishing the difference in volume loss and checking the modeling behaviour.

5) Investigating the carbides and intermetallics in the alloys further by using EPMA (Electron probe micro-analyzer) equipped with WDS (wavelength-dispersive spectroscopy). 


\section{Appendix Summary of Raw Data}

\section{AP-1 Continuous Testing}

Table AP- 1: Raw data of wear track measurements for Stellite 21 continuous tests $50 \mathrm{rpm}$

\begin{tabular}{|l|c|c|c|c|c|c|}
\cline { 2 - 5 } \multicolumn{1}{c|}{} & \multicolumn{4}{c|}{ Cross-sectional area $\left(\boldsymbol{\mu m}^{2}\right)$} & \multirow{2}{*}{$\begin{array}{c}\text { Standard } \\
\text { Deviation }\end{array}$} & \multirow{2}{*}{ Mean $\left(\boldsymbol{\mu m}^{\mathbf{2}}\right)$} \\
\hline Test Condition & Point 1 & Point 2 & Point 3 & Point 4 & \\
\hline 15 N 10 hrs & 20796 & 33768 & 37048 & 27989 & 6176.4 & 29900.3 \\
\hline 15 N 20 hrs & 30791 & 25818 & 23070 & 24093 & 2966.3 & 25943.0 \\
\hline 15 N 40 hrs & 40244 & 48124 & 39298 & 42692 & 3427.0 & 42589.5 \\
\hline 25 N 10 hrs & 56264 & 62147 & 38849 & 69659 & 11362.8 & 56729.8 \\
\hline 25 N 20 hrs & 129461 & 154814 & 156657 & 137199 & 11550.4 & 144532.8 \\
\hline 25 N 40 hrs & 304892 & 224885 & 215649 & 38094 & 97483.5 & 195880.0 \\
\hline
\end{tabular}

Table AP- 2: Raw data of wear track measurements for Stellite 720 continuous tests $50 \mathrm{rpm}$

\begin{tabular}{|l|c|c|c|c|c|c|}
\cline { 2 - 5 } \multicolumn{1}{c|}{} & \multicolumn{4}{c|}{ Cross-sectional area $\left(\boldsymbol{\mu m}^{2}\right)$} & \multirow{2}{*}{$\begin{array}{c}\text { Standard } \\
\text { Deviation }\end{array}$} & \multirow{2}{*}{ Mean $\left(\boldsymbol{\mu m}^{\mathbf{2}}\right)$} \\
\hline Test Condition & Point 1 & Point 2 & Point 3 & Point 4 & \\
\hline 15 N 10 hrs & 2779 & 1230 & 2182 & 1864 & 651.7 & 1265.0 \\
\hline 15 N 20 hrs & 10442 & 13936 & 8755 & 8289 & 2217.0 & 10355.5 \\
\hline 15 N 40 hrs & 2291 & 2132 & 2077 & 936 & 417.6 & 1859.0 \\
\hline 25 N 10 hrs & 9224 & 13679 & 11579 & 9120 & 1881.6 & 10900.5 \\
\hline 25 N 20 hrs & 14234 & 12923 & 12494 & 9182 & 1861.1 & 12208.3 \\
\hline 25 N 40 hrs & 11556 & 14017 & 11224 & 10163 & 1411.7 & 11740.0 \\
\hline
\end{tabular}

Table AP- 3: Raw data of wear track measurements for Stellite 728 continuous tests $50 \mathrm{rpm}$

\begin{tabular}{|c|c|c|c|c|c|c|}
\hline \multirow[b]{2}{*}{ Test Condition } & \multicolumn{4}{|c|}{ Cross-sectional area $\left(\mu \mathrm{m}^{2}\right)$} & \multirow{2}{*}{$\begin{array}{l}\text { Standard } \\
\text { Deviation }\end{array}$} & \multirow{2}{*}{ Mean $\left(\mu \mathrm{m}^{2}\right)$} \\
\hline & Point 1 & Point 2 & Point 3 & Point 4 & & \\
\hline $15 \mathrm{~N} 10 \mathrm{hrs}$ & 30167 & 31540 & 33484 & 29390 & 1554.3 & 31145.3 \\
\hline 15 N 20 hrs & 7377 & 7326 & 5324 & 6943 & 836.0 & 6742.5 \\
\hline $15 \mathrm{~N} 40 \mathrm{hrs}$ & 12755 & 10676 & 13635 & 11624 & 1120.1 & 12172.5 \\
\hline $25 \mathrm{~N} 10 \mathrm{hrs}$ & 27153 & 44945 & 42945 & 25784 & 8780.2 & 35206.8 \\
\hline $25 \mathrm{~N} 20 \mathrm{hrs}$ & 39178 & 53637 & 46878 & 44698 & 5179.1 & 46097.8 \\
\hline $25 \mathrm{~N} 40 \mathrm{hrs}$ & 46151 & 53943 & 40919 & 39466 & 5668.4 & 45119.8 \\
\hline
\end{tabular}


Table AP- 4: Summary of volume loss for all continuous tests $50 \mathrm{rpm}$

\begin{tabular}{|c|c|c|c|c|c|}
\hline Alloy & Force $(\mathbf{N})$ & Time (hr) & Total volume loss $\left(\mathrm{mm}^{3}\right)$ & $\begin{array}{l}\text { Mean } \\
\left(\mathrm{mm}^{3}\right)\end{array}$ & $\begin{array}{l}\text { Standard } \\
\text { deviation }\end{array}$ \\
\hline \multirow{10}{*}{21} & \multirow{6}{*}{15} & 10 & 1.428 & \multirow{2}{*}{1.184} & \multirow{2}{*}{0.244} \\
\hline & & 10 & 0.939 & & \\
\hline & & 20 & 2.069 & \multirow{2}{*}{1.442} & \multirow{2}{*}{0.627} \\
\hline & & 20 & 0.815 & & \\
\hline & & 40 & 1.215 & & \\
\hline & & 40 & 1.338 & 1.276 & 0.062 \\
\hline & \multirow{4}{*}{25} & 10 & 1.782 & \multirow{2}{*}{1.756} & \multirow{2}{*}{0.027} \\
\hline & & 10 & 1.729 & & \\
\hline & & 20 & 4.541 & N/A & $\mathrm{N} / \mathrm{A}$ \\
\hline & & 40 & 6.155 & N/A & N/A \\
\hline \multirow{10}{*}{720} & \multirow{5}{*}{15} & 10 & 0.143 & \multirow{2}{*}{0.101} & \multirow{2}{*}{0.042} \\
\hline & & 10 & 0.059 & & \\
\hline & & 20 & 0.325 & N/A & N/A \\
\hline & & 40 & 0.075 & \multirow{2}{*}{0.066} & \multirow{2}{*}{0.008} \\
\hline & & 40 & 0.058 & & \\
\hline & \multirow{5}{*}{25} & 10 & 0.342 & \multirow{3}{*}{0.272} & \multirow{3}{*}{0.125} \\
\hline & & 10 & 0.377 & & \\
\hline & & 10 & 0.095 & & \\
\hline & & 20 & 0.384 & $\mathrm{~N} / \mathrm{A}$ & N/A \\
\hline & & 40 & 0.369 & N/A & N/A \\
\hline \multirow{12}{*}{728} & \multirow{8}{*}{15} & 10 & 0.979 & \multirow{2}{*}{0.881} & \multirow{2}{*}{0.098} \\
\hline & & 10 & 0.783 & & \\
\hline & & 20 & 0.212 & \multirow{3}{*}{0.536} & \multirow{3}{*}{0.283} \\
\hline & & 20 & 0.902 & & \\
\hline & & 20 & 0.494 & & \\
\hline & & 40 & 0.168 & \multirow{3}{*}{0.358} & \multirow{3}{*}{0.146} \\
\hline & & 40 & 0.382 & & \\
\hline & & 40 & 0.523 & & \\
\hline & \multirow{4}{*}{25} & 10 & 1.106 & \multirow{2}{*}{0.943} & \multirow{2}{*}{0.163} \\
\hline & & 10 & 0.780 & & \\
\hline & & 20 & 1.448 & N/A & N/A \\
\hline & & 40 & 1.418 & N/A & N/A \\
\hline
\end{tabular}

Note: Highlighted rows were selected for modeling analysis 


\section{AP-2 Interrupted Testing}

Table AP- 5: Raw data of wear track measurements for Stellite 21 interrupted tests $50 \mathrm{rpm}$

\begin{tabular}{|c|c|c|c|c|c|c|}
\hline \multirow[b]{2}{*}{ Test Condition } & \multicolumn{4}{|c|}{ Cross-sectional area $\left(\mu \mathrm{m}^{2}\right)$} & \multirow{2}{*}{$\begin{array}{l}\text { Standard } \\
\text { Deviation }\end{array}$} & \multirow{2}{*}{ Mean $\left(\mu \mathrm{m}^{2}\right)$} \\
\hline & Point 1 & Point 2 & Point 3 & Point 4 & & \\
\hline $25 \mathrm{~N} 1 \mathrm{hr}$ & 4122 & 4862 & 4905 & 5006 & 351.3 & 4723.8 \\
\hline $25 \mathrm{~N} 3 \mathrm{hrs}$ & 14067 & 12366 & 10849 & 13548 & 1237.5 & 12707.5 \\
\hline $25 \mathrm{~N} 5 \mathrm{hrs}$ & 28020 & 24153 & 16654 & 20384 & 4233.8 & 22302.8 \\
\hline $25 \mathrm{~N} 7 \mathrm{hrs}$ & 23033 & 30724 & 38052 & 38571 & 6332.9 & 32595.0 \\
\hline $25 \mathrm{~N} 11 \mathrm{hrs}$ & 51527 & 66054 & 68724 & 50506 & 8248.4 & 59202.8 \\
\hline $25 \mathrm{~N} 15 \mathrm{hrs}$ & 53282 & 42194 & 58316 & 63268 & 7812.5 & 54265.0 \\
\hline $25 \mathrm{~N} 20 \mathrm{hrs}$ & 50546 & 66308 & 70360 & 51969 & 8672.2 & 59795.8 \\
\hline $25 \mathrm{~N} 30 \mathrm{hrs}$ & 68369 & 70971 & 51908 & 43862 & 11295.4 & 58777.5 \\
\hline $25 \mathrm{~N} 40 \mathrm{hrs}$ & 65403 & 68715 & 59244 & 52146 & 6320.9 & 61377.0 \\
\hline
\end{tabular}

Table AP- 6: Raw data of wear track measurements for Stellite 720 interrupted tests $50 \mathrm{rpm}$

\begin{tabular}{|c|c|c|c|c|c|c|}
\hline \multirow[b]{2}{*}{ Test Condition } & \multicolumn{4}{|c|}{ Cross-sectional area $\left(\mu \mathrm{m}^{2}\right)$} & \multirow{2}{*}{$\begin{array}{l}\text { Standard } \\
\text { Deviation }\end{array}$} & \multirow{2}{*}{ Mean $\left(\mu \mathrm{m}^{2}\right)$} \\
\hline & Point 1 & Point 2 & Point 3 & Point 4 & & \\
\hline $25 \mathrm{~N} 1 \mathrm{hr}$ & 3519 & 3132 & 3093 & 3255 & 166.6 & 3249.8 \\
\hline $25 \mathrm{~N} 3 \mathrm{hrs}$ & 3287 & 3041 & 5536 & 6774 & 1560.7 & 4659.5 \\
\hline $25 \mathrm{~N} 5 \mathrm{hrs}$ & 4414 & 2966 & 5129 & 3345 & 857.2 & 3963.5 \\
\hline $25 \mathrm{~N} 7 \mathrm{hrs}$ & 4465 & 4765 & 5607 & 3528 & 743.0 & 4591.3 \\
\hline $25 \mathrm{~N} 9 \mathrm{hrs}$ & 3967 & 3730 & 5606 & 4210 & 728.9 & 4378.3 \\
\hline $25 \mathrm{~N} 11 \mathrm{hrs}$ & 5924 & 4528 & 4575 & 4054 & 696.6 & 4770.3 \\
\hline $25 \mathrm{~N} 15 \mathrm{hrs}$ & 3686 & 4564 & 4182 & 5871 & 810.0 & 4575.8 \\
\hline $25 \mathrm{~N} 17 \mathrm{hrs}$ & 4436 & 3517 & 6686 & 3125 & 1380.7 & 4441.0 \\
\hline $25 \mathrm{~N} 40 \mathrm{hrs}$ & 4092 & 4249 & 4319 & 6646 & 1053.7 & 4826.5 \\
\hline
\end{tabular}


Table AP- 7: Raw data of wear track measurements for Stellite 728 interrupted tests 50rpm

\begin{tabular}{|l|c|c|c|c|c|c|}
\cline { 2 - 5 } \multicolumn{1}{c|}{} & \multicolumn{4}{c|}{ Cross-sectional area $\left(\boldsymbol{\mu m}^{\mathbf{2}}\right)$} & \multirow{2}{*}{$\begin{array}{c}\text { Standard } \\
\text { Deviation }\end{array}$} & \multirow{2}{*}{ Mean $\left.\mathbf{( \mu m}^{\mathbf{2}}\right)$} \\
\hline Test Condition & Point 1 & Point 2 & Point 3 & Point 4 & & \\
\hline 25 N 1hr & 3324 & 2782 & 3027 & 2804 & 218.3 & 2984.3 \\
\hline 25 N 3 hrs & 7239 & 10261 & 8607 & 7357 & 1218.3 & 8366.0 \\
\hline 25 N 5 hrs & 15018 & 13135 & 13971 & 16478 & 1248.3 & 14650.5 \\
\hline 25 N 7 hrs & 18717 & 23535 & 19198 & 20052 & 1885.8 & 20375.5 \\
\hline 25 N 9 hrs & 24690 & 25286 & 29178 & 29986 & 2324.3 & 27285.0 \\
\hline 25 N 11 hrs & 22424 & 26052 & 29137 & 31093 & 3280.0 & 27176.5 \\
\hline 25 N 13 hrs & 25092 & 30152 & 28940 & 26905 & 1934.1 & 27772.3 \\
\hline 25 N 15 hrs & 29582 & 29153 & 26685 & 25886 & 1574.0 & 27826.5 \\
\hline 25 N 17 hrs & 6226 & 28348 & 25565 & 22719 & 8598.4 & 20714.5 \\
\hline 25 N 30 hrs & 30258 & 23164 & 27630 & 30328 & 2913.2 & 27845.0 \\
\hline 25 N 40 hrs & 27408 & 31423 & 30107 & 27058 & 1830.5 & 28999.0 \\
\hline
\end{tabular}

Table AP- 8: Raw data of wear track measurements for Stellite 21 interrupted tests $60 \mathrm{rpm}$

\begin{tabular}{|c|c|c|c|c|c|c|}
\hline & \multicolumn{4}{|c|}{ Cross-sectional area $\left(\mu \mathrm{m}^{2}\right)$} & \multirow{2}{*}{$\begin{array}{l}\text { Standard } \\
\text { Deviation }\end{array}$} & \multirow{2}{*}{ Mean $\left(\mu \mathrm{m}^{2}\right)$} \\
\hline Test Condition & Point 1 & Point 2 & Point 3 & Point 4 & & \\
\hline $25 \mathrm{~N} 10 \mathrm{hr}$ & 61747 & 38232 & 47260 & 68081 & 11749.8 & 53830.0 \\
\hline $25 \mathrm{~N} 12 \mathrm{hrs}$ & 51199 & 72375 & 70562 & 62845 & 8339.1 & 64245.3 \\
\hline $25 \mathrm{~N} 14 \mathrm{hrs}$ & 70365 & 58938 & 51320 & 71665 & 8399.8 & 63072.0 \\
\hline $25 \mathrm{~N} 20 \mathrm{hrs}$ & 55658 & 73590 & 74094 & 55433 & 9150.3 & 64693.8 \\
\hline $25 \mathrm{~N} 40 \mathrm{hrs}$ & 57099 & 72278 & 75764 & 46391 & 11828.2 & 62883.0 \\
\hline $25 \mathrm{~N} 60 \mathrm{hrs}$ & 81329 & 78938 & 56268 & 64234 & 10367.0 & 70192.3 \\
\hline
\end{tabular}


Table AP- 9: Raw data of wear track measurements for Stellite 720 interrupted tests $60 \mathrm{rpm}$

\begin{tabular}{|c|c|c|c|c|c|c|}
\hline & \multicolumn{4}{|c|}{ Cross-sectional area $\left(\mu \mathrm{m}^{2}\right)$} & \multirow{2}{*}{$\begin{array}{l}\text { Standard } \\
\text { Deviation }\end{array}$} & \multirow{2}{*}{ Mean $\left(\mu \mathrm{m}^{2}\right)$} \\
\hline Test Condition & Point 1 & Point 2 & Point 3 & Point 4 & & \\
\hline $25 \mathrm{~N} 1 \mathrm{hr}$ & 2099 & 3736 & 1588 & 2390 & 794.3 & 2453.3 \\
\hline $25 \mathrm{~N} 3 \mathrm{hrs}$ & 6047 & 12464 & 10433 & 7877 & 2442.6 & 9205.3 \\
\hline $25 \mathrm{~N} 5 \mathrm{hrs}$ & 19914 & 12394 & 12320 & 14928 & 3085.3 & 14889.0 \\
\hline $25 \mathrm{~N} 7 \mathrm{hrs}$ & 25016 & 15497 & 13657 & 20772 & 4468.5 & 18735.5 \\
\hline $25 \mathrm{~N} 9 \mathrm{hrs}$ & 28902 & 15708 & 13718 & 24516 & 6235.0 & 20711.0 \\
\hline $25 \mathrm{~N} 10 \mathrm{hrs}$ & 29537 & 15952 & 17068 & 21420 & 5338.5 & 20994.3 \\
\hline $25 \mathrm{~N} 12 \mathrm{hrs}$ & 10575 & 31757 & 15572 & 14943 & 8053.7 & 18211.8 \\
\hline $25 \mathrm{~N} 14 \mathrm{hrs}$ & 14724 & 25550 & 18557 & 28211 & 5379.2 & 21760.5 \\
\hline $25 \mathrm{~N} 16 \mathrm{hrs}$ & 26186 & 16491 & 15441 & 25563 & 4973.0 & 20920.3 \\
\hline $25 \mathrm{~N} 18 \mathrm{hrs}$ & 16116 & 16948 & 25923 & 19604 & 3845.2 & 19647.8 \\
\hline $25 \mathrm{~N} 24 \mathrm{hrs}$ & 21081 & 21803 & 16384 & 17961 & 2221.1 & 19307.3 \\
\hline $25 \mathrm{~N} 35 \mathrm{hrs}$ & 19412 & 19196 & 19988 & 20589 & 541.6 & 19796.3 \\
\hline $25 \mathrm{~N} 40 \mathrm{hrs}$ & 18757 & 29282 & 21674 & 17277 & 4628.8 & 21747.5 \\
\hline $25 \mathrm{~N} 60 \mathrm{hrs}$ & 19515 & 25755 & 30553 & 26445 & 3946.3 & 25567.0 \\
\hline
\end{tabular}

Table AP- 10: Raw data of wear track measurements for Stellite 728 interrupted tests 60rpm

\begin{tabular}{|c|c|c|c|c|c|c|}
\cline { 2 - 5 } \multicolumn{1}{c|}{} & \multicolumn{3}{c|}{ Cross-sectional area $\left.\mathbf{( \mu m}^{2}\right)$} & \multirow{2}{*}{$\begin{array}{c}\text { Standard } \\
\text { Deviation }\end{array}$} & \multirow{2}{*}{ Mean $\left.\boldsymbol{( \mu m}^{\mathbf{2}}\right)$} \\
\hline Test Condition & Point 1 & Point 2 & Point 3 & Point 4 & \\
\hline 25 N 1 hr & 3291 & 2743 & 3622 & 3174 & 314.5 & 3207.5 \\
\hline 25 N 3 hrs & 9861 & 11668 & 8842 & 8962 & 1130.1 & 9833.3 \\
\hline 25 N 5 hrs & 16425 & 19449 & 14596 & 17865 & 1790.8 & 17083.8 \\
\hline 25 N 7 hrs & 16539 & 19118 & 18096 & 19988 & 1283.4 & 18435.3 \\
\hline 25 N 9 hrs & 18850 & 19531 & 21012 & 18867 & 879.4 & 19565.0 \\
\hline 25 N 11 hrs & 15994 & 23586 & 21892 & 21431.5 & 3255.3 & 21431.5 \\
\hline 25 N 20 hrs & 19259 & 21399 & 21808 & 24384 & 1821.0 & 21712.5 \\
\hline 25 N 30 hrs & 24254 & 32857 & 32973 & 23041 & 4049.1 & 29404.3 \\
\hline 25 N 40 hrs & 19259 & 35907 & 33299 & 35508 & 1294.3 & 35508.0 \\
\hline 25 N 60 hrs & 28746 & 35893 & 34415 & 35610 & 608.4 & 35450.3 \\
\hline
\end{tabular}




\section{Bibliography}

[1] J. R. Davis, ASM Specialty Handbook: Nickel, Cobalt, and Their Alloys. ASM International, 2000.

[2] S. (Ed) Zhang and D. (Ed) Zhao, Aerospace Materials Handbook. CRC Press, 2013.

[3] R. Liu, J. H. Yao, Q. L. Zhang, M. X. Yao, and R. Collier, "Microstructures and Hardness/Wear Performance of High-Carbon Stellite Alloys Containing Molybdenum," Metall. Mater. Trans. A Phys. Metall. Mater. Sci., vol. 46, no. 12, pp. 5504-5513, 2015.

[4] M. X. Yao, and R. Collier, "Improved Corrosion and Wear Resistance of a Niobium Alloyed Co-Cr-Mo Alloy," NACE Int. Conf., no. 5960, pp. 1-15, 2015.

[5] R. P. J. Blau, and B. T. Consulting, "Sliding and Adhesive Wear," Frict. Lubr. Wear Technol., vol. 18, pp. 313-322, 2017.

[6] P. Crook, "Cobalt and Cobalt Alloys," ASM Handb. Vol. 2 Prop. Sel. Nonferrous Alloy. Spec. Mater., vol. 2, pp. 446-454, 1990.

[7] ASM, Volume 18, Friction, Lubrication, and Wear Technology. 1995.

[8] R. Ahmed, and H. L. De Villiers Lovelock, "Friction and Wear of Cobalt-Base Alloys," Frict. Lubr. Wear Technol., vol. 18, pp. 487-501, 2017.

[9] C. Guyard, A. Barbangelo, C. H. Allibert, and J. Driole, "Solidification Path and Phase Equilibria in the Liquid-Solid Range of Cobalt-Base Alloy," J. Mater. Sci., vol. 16, no. 3, pp. 604-612, 1981.

[10] C. Lund, and H. Wagner, "Identification of Microconstituents in Superalloys," Def. Met. Inf. Center, Battelle Meml. InsitituteD, no. AD289 664, 1962.

[11] M. J. Donachie, and S. J. Donachie, "Understanding Superalloy Metallurgy," Superalloys A Tech. Guid., pp. 25-39, 2002.

[12] H. Yu, R. Ahmed, and H. de Villiers Lovelock, "A Comparison of the Tribo-Mechanical Properties of a Wear Resistant Cobalt-Based Alloy Produced by Different Manufacturing Processes," J. Tribol., vol. 129, no. 3, p. 586, 2007.

[13] D. Li, “Abrasive Wear,” Frict. Lubr. Wear Technol., vol. 18, no. c, pp. 243-251, 2017.

[14] K. Zum Gahr, "Microstructure and Wear of Materials." Elsevier B.V., 1987.

[15] P. J. Alison, and H. Wilman, "The Different Behaviour of Hexagonal and Cubic Metals in their Friction, Wear and Work Hardening during Abrasion," Br. J. Appl. Phys., vol. 15, no. 3, pp. 281-289, 1964.

[16] R. P. Steijn, "Friction and Wear of Single Crystals," Wear, vol. 7, no. I, pp. 48-66, 1964.

[17] W. L. Silence, "Effect of Structure on Wear Resistance of Co-, Fe-, and Ni-Base Alloys," J. Lubr. Technol., vol. 100, no. 3, pp. 428-435, 1978.

[18] P. J. Blau, "On the Nature of Running-In," Tribol. Int., vol. 38, no. 11-12 SPEC. ISS., pp. 1007-1012, 2005.

[19] G. C. Wood, and F. H. Stott, "The Influence of Oxides on the Friction and Wear of Alloys," Tribol. Int., vol. 11, no. August, pp. 211-218, 1978.

[20] I. A. Inman, S. R. Rose, and P. K. Datta, "Studies of High Temperature Sliding Wear of Metallic Dissimilar Interfaces II: Incoloy MA956 versus Stellite 6," Tribol. Int., vol. 39, no. 11, pp. 1361-1375, 2006.

[21] J. F. Archard, "Contact and Rubbing of Flat Surfaces," J. Appl. Phys., vol. 24, no. 8, pp. 981-988, 1953.

[22] J. F. Archard, and W. Hirst, "The Wear of Metals Under Unlubricated Conditions," Proc. R. Soc. London. Ser. A, Math. Phys. Sci., vol. 236, no. 1206, pp. 397-410, 1956. 
[23] I. M. Hutchings, Tribology - Friction and Wear of Materials. Great Britain: Edward Arnold, 1992.

[24] A. D. Sarkar, Friction and Wear. London: Academic Press, 1980.

[25] V. Hegadekatte, S. Kurzenhäuser, N. Huber, and O. Kraft, "A Predictive Modeling Scheme for Wear in Tribometers," Tribol. Int., vol. 41, no. 11, pp. 1020-1031, 2008.

[26] R. McCarron, D. Stewart, P. Shipway, and D. Dini, "Sliding Wear Analysis of Cobalt Based Alloys in Nuclear Reactor Conditions," Wear, vol. 376-377, pp. 1489-1501, 2017.

[27] Zhenfang Zhang, Liangchi Zhang, Yiu-Wing Mai, Z. Zhang, L. Zhang, and Y. Mai, "The Running-In Wear of a Steel/SiC p-Al Composite System," Wear, vol. 194, pp. 38-43, 2014.

[28] K. C. Antony, "Wear-Resistant Cobalt-Base Alloys," J. Met., no. February, pp. 52-60, 1983.

[29] R. E. Reed-Hill, Physical Metallurgy Principles, 2nd ed. Brooks/Cole Engineering Division, Monterey, California 93940, 1972.

[30] A. Frenk, "Laser CLadding with Cobalt-Based Hardfacing Alloys," J. Phys. IV Colloq., vol. 1 (C7), pp. C7-65-C7-68, 1991.

[31] A. Frenk, and W. Kurz, "Microstructural Effects on the Sliding Wear Resistance of a Cobalt- Based Alloy," Wear, vol. 174, pp. 81-91, 1994.

[32] M. X. Yao, J. B. C. Wu, and Y. Xie, "Wear, Corrosion and Cracking Resistance of some W- or Mo-Containing Stellite Hardfacing Alloys," Mater. Sci. Eng. A, vol. 407, no. 1-2, pp. 234-244, 2005.

[33] E. Goldstein, J.I., Newbury, D.E., Echlin, P., Joy, D.C., Romig Jr, A.D., Lyman, C.E., Firori, C., Lifshin, Scanning Electron Microscopy and X-Ray Microanalysis. Plenum Press, New York, 1992.

[34] Stellite.com., "Wear Solutions Components", 2013. Available at: http://www.stellite.com/en/products/components.html

[35] J. Yao, Y. Ding, R. Liu, Q. Zhang, and L. Wang, "Wear and Corrosion Performance of Laser-Clad Low-Carbon High- Molybdenum Stellite alloys," Opt. Laser Technol., vol. 107, pp. 32-45, 2018.

[36] J. F. Archard, W. Hirst, "An Examination of a Mild Wear Process," Proc. R. Soc. London . Ser. A , Math. Phys., vol. 238, no. 1215, pp. 515-528, 1957.

[37] U. Malayoglu, and A. Neville, "Mo and W as Alloying Elements in Co-based Alloys - Their Effects on Erosion-Corrosion Resistance," Wear, vol. 259, no. 1-6, pp. 219-229, 2005.

[38] R. Ahmed, H. L. De Villiers Lovelock, N. H. Faisal, and S. Davies, "Structure-Property Relationships in a CoCrMo Alloy at Micro and Nano-Scales," Tribol. Int., vol. 80, pp. 98$114,2014$.

[39] R. Liu, J. H. Yao, Q. L. Zhang, M. X. Yao, and R. Collier, "Sliding Wear and Solid-Particle Erosion Resistance of a Novel High-Tungsten Stellite alloy," Wear, vol. 322-323, pp. 4150, 2015.

[40] M. F. Amateau, and W. A. Glaeser, "Survey of Materials for High-Temperature Bearing and Sliding Applications," Wear, vol. 7, no. 5, pp. 385-418, 1964.

[41] S. Kapoor, "High-Temperature Hardness and Wear Resistance of Stellite Alloys", Master Degree Thesis, Carleton University, Ottawa, Canada, 2012.

[42] L. J. Yang, "The Transient and Steady Wear Coefficients of A6061 Aluminium Alloy Reinforced with Alumina Particles," Compos. Sci. Technol., vol. 63, no. 3-4, pp. 575-583, 2003. 
[43] V. Popov, Contact Mechanics and Friction. Springer-Verlag Berlin Heidelberg, 2010.

[44] S. T. Method, "Standard Test Method for Wear Testing with a Pin-on-Disk Apparatus 1," Wear, vol. 05, no. Reapproved 2010, pp. 1-5, 2011.

[45] P. J. Blau, M. Yao, J. Qu, and J. Wu, "Use of Multiple Criteria to Map the High-Temperature Scuffing Behavior of Co-Based Superalloys," Wear, vol. 267, no. 1-4, pp. 374-379, 2009.

[46] M. F. Ashby, and S. C. Lim, "Wear-Mechanism Maps," Scr. Metall. Mater., vol. 24, no. 5, pp. 805-810, 1990.

[47] S. T. Method, "Standard Test Method for Wear Testing with a Pin-on-Disk Apparatus 1," Wear, vol. 05, no. Reapproved 2010, pp. 1-5, 2011.

[48] A. V. Vidyapeetham and N. Radhika, "Optimisation of Dry Sliding Wear Process Parameters for Aluminium Hybrid Metal Tribology in Industry," Tribol. Ind., vol. 36, no. 2, 2014.

[49] L. Yang, "Prediction of Steady-State Wear Coefficients in Adhesive Wear," Tribol. Trans., vol. 47, no. 3, pp. 335-340, 2004.

[50] A. Rastkar, and T. Bell, "Tribological Performance of Plasma Nitrided Gamma Based Titanium Aluminides," Wear, vol. 253, no. 11-12, pp. 1121-1131, 2002.

[51] M. Scherge, D. Shakhvorostov, and K. Pöhlmann, "Fundamental Wear Mechanisms of Metals," Wear, vol. 255, pp. 395-400, 2003.

[52] L. J. Yang, "The Effect of Nominal Specimen Contact Area on the Wear Coefficient of A6061 Aluminium Matrix Composite Reinforced with Alumina Particles," Wear, vol. 263, no. 7-12 SPEC. ISS., pp. 939-948, 2007.

[53] L. J. Yang, "Wear Coefficient Equation for Aluminium-Based Matrix Composites against Steel Disc," Wear, vol. 255, no. 1-6, pp. 579-592, 2003.

[54] Z. Zhang, L. Zhang, and Y. Mai, "The Running-In Wear of a Steel/SiC p-Al Composite System," Wear, vol. 194, pp. 38-43, 2014.

[55] A. Frenk, and W. Kurz, "Microstructural Effects on the Sliding Wear Resistance of a Cobalt- Based Alloy," Wear, vol. 174, pp. 81-91, 1994.

[56] L. J. Yang, "A Test Methodology for the Determination of Wear Coefficient," Wear, vol. 259, no. 7-12, pp. 1453-1461, 2005.

[57] J. F. Archard, "Contact and Rubbing of Flat Surfaces," J. Appl. Phys., vol. 24, no. 8, pp. 981-988, 1953.

[58] L. J. Yang, "A Methodology for the Prediction of Standard Steady-State Wear Coefficient in an Aluminium-Based Matrix Composite Reinforced with Alumina Particles," J. Mater. Process. Technol., vol. 162-163, no. SPEC. ISS., pp. 139-148, 2005.

[59] I. Radu, D. Y. Li, and R. Llewellyn, "Tribological Behavior of Stellite 21 Modified with Yttrium," Wear, vol. 257, no. 11, pp. 1154-1166, 2004.

[60] J. C. Shin, J. M. Doh, J. K. Yoon, D. Y. Lee, and J. S. Kim, "Effect of Molybdenum on the Microstructure and Wear Resistance of Cobalt-Base Stellite Hardfacing alloys," Surf. Coatings Technol., vol. 166, no. 2-3, pp. 117-126, 2003.

[61] S. C. Lim, and M. F. Ashby, "Overview no. 55 Wear-Mechanism Maps," Acta Metall., vol. 35, no. 1, pp. 1-24, 1987.

[62] M. A. Ashraf, R. Ahmed, O. Ali, N. H. Faisal, A. M. El-Sherik, and M. F. A. Goosen, "Finite Element Modeling of Sliding Wear in a Composite Alloy Using a Free-Mesh," $J$. Tribol., vol. 137, no. 3, p. 031605, 2015. 
[63] M. F. Rothman, R. D. Zordan, and D. Muzyka, "Role of Refractory Elements in CobaltBase Alloys." ASM Conference on Refractory Alloying Elements in Superalloys - Effects and Availability, Rio de Janeiro, pp 101-115, 1984.

[64] I. A. Inman, and P. S. Datta, "Development of a Simple 'Temperature Versus Sliding Speed' Wear Map for the Sliding Wear Behaviour of Dissimilar Metallic Interfaces II," Wear, vol. 265, no. 11-12, pp. 1592-1605, 2008.

[65] R. P. J. Blau, and B. T. Consulting, "Sliding and Adhesive Wear" Frict. Lubr. Wear Technol., vol. 18, pp. 313-322, 2017.

[66] L. Yang, "Prediction of Steady-State Wear Coefficients in Adhesive Wear," Tribol. Trans., vol. 47, no. 3, pp. 335-340, 2004.

[67] R. Liu, J. Yao, Q. Zhang, M. X. Yao, and R. Collier, "Effects of Molybdenum Content on the Wear/Erosion and Corrosion Performance of Low-Carbon Stellite Alloys," Mater. Des., vol. 78, pp. 95-106, 2015.

[68] J. F. Archard, "Friction between Metal Surfaces," Wear, vol. 113, no. 1, pp. 3-16, 1986.

[69] J. F. Archard, "Surface Topography and Tribology," Tribol. Int., vol. 7, no. 5, pp. 213-220, 1974.

[70] M. E. Sikorski, "The Adhesion of Metals and Factors that Influence it," Wear, vol. 7, no. 2, pp. 144-162, 1964.

[71] R. Ahmed, H. L. De Villiers Lovelock, S. Davies, and N. H. Faisal, "Influence of Re-HIPing on the Structure-Property relationships of Cobalt-Based Alloys," Tribol. Int., vol. 57, pp. 8-21, 2013. 